

\section{Changing perspectives}

Towards detailed phenotyping in genetics

Mats Nagel 
(C) Mats Nagel, 2020

All rights reserved.

The research in this thesis was supported by a grant from the Netherlands Organization for Scientific Research, project number 452-12-014.

Cover design: Mats Nagel

Cover photo: Vern Dewit

Printing: Off Page

ISBN: 978-94-93197-07-7

DOI: 10.31237/osf.io/a4nz2 


\title{
Changing perspectives
}

\section{Towards detailed phenotyping in genetics}

\author{
ACADEMISCH PROEFSCHRIFT
}

ter verkrijging van de graad Doctor of Philosophy aan

de Vrije Universiteit Amsterdam,

op gezag van de rector magnificus

prof.dr. V. Subramaniam,

in het openbaar te verdedigen

ten overstaan van de promotiecommissie

van de Faculteit der Bètawetenschappen

op dinsdag 30 juni 2020 om 13.45 uur

in een online bijeenkomst van de universiteit,

De Boelelaan 1105

door

Mats Nagel

geboren te Amsterdam 
promotor:

copromotor: prof.dr. D.P. Posthuma

dr. S. van der Sluis 
Leescommissie: prof.dr. H. D. Mansvelder

prof. C. M. Lewis

prof.dr. B. W. J. H. Penninx

dr. E. I. Fried

dr. M. G. Nivard

Paranimfen: $\quad$ Oscar van Mourik

Philip R. Jansen 


\section{Contents}

1 General introduction 9

$\begin{array}{ll}1.1 \text { Brief introduction } & 10\end{array}$

$\begin{array}{ll}1.2 \text { Background } & 11\end{array}$

1.3 Genetic research into psychological traits 15

1.4 Aims and outline of this thesis 24

$\begin{array}{ll}1.5 \text { Glossary } & 27\end{array}$

2 Item-level analyses reveal genetic heterogeneity in neuroticism 31

Abstract 32

2.1 Introduction 33

2.2 Results 34

2.3 Discussion 43

2.4 Methods $\quad 44$

2.5 Supplementary information $\quad 50$

3 Meta-analysis of genome-wide association studies for neuroticism in 449,484 individuals identifies novel genetic loci and pathways 53

Abstract $\quad 54$

3.1 Results $\quad 55$

$\begin{array}{ll}3.2 \text { Discussion } & 68\end{array}$

$\begin{array}{ll}3.3 \text { Methods } & 69\end{array}$

$\begin{array}{ll}3.4 \text { Supplementary methods } & 78\end{array}$

$\begin{array}{ll}3.5 \text { Supplementary results } & 83\end{array}$

4 Examining the genetic validity of internalizing disorders through

item-level analyses 99

$\begin{array}{ll}\text { Abstract } & 100\end{array}$

$\begin{array}{ll}4.1 \text { Introduction } & 101\end{array}$

$\begin{array}{ll}4.2 \text { Results } & 102\end{array}$

$\begin{array}{ll}4.3 \text { Discussion } & 110\end{array}$

$\begin{array}{ll}4.4 \text { Methods } & 113\end{array}$

$\begin{array}{ll}\text { 4.5 Supplementary methods } & 121\end{array}$ 
5 Genome-wide meta-analysis of brain volume on 47,316 individuals identifies genomic loci and genes shared with intelligence $\quad 125$

$\begin{array}{ll}\text { Abstract } & 126\end{array}$

5.1 Introduction 127

$\begin{array}{ll}5.2 \text { Results } & 127\end{array}$

5.3 Conclusion 136

$\begin{array}{ll}5.4 \text { Methods } & 138\end{array}$

5.5 Supplementary methods $\quad 147$

5.6 Supplementary results 148

$\begin{array}{lll}6 & \text { Discussion } & 155\end{array}$

6.1 Background 156

6.2 Summary of findings $\quad 157$

6.3 Obstacles in the way of studying detailed phenotypes 159

$\begin{array}{ll}6.4 \text { Future directions } & 162\end{array}$

$\begin{array}{ll}\text { References } & 167\end{array}$

$\begin{array}{ll}\text { Peer-reviewed publications } & 183\end{array}$

$\begin{array}{ll}\text { Nederlandse samenvatting } & 185\end{array}$

$\begin{array}{ll}\text { Dankwoord } & 187\end{array}$ 
1 General introduction 


\subsection{Brief introduction}

Mental health problems are highly prevalent in modern-day society ${ }^{1}$. Problems range from nonclinical manifestations of disturbed mood to severe, chronic psychiatric disorders like major depression, schizophrenia and autism spectrum disorder (ASD). Despite several decades of intensive research aimed at identifying the underlying biological mechanisms, and potential drug targets, pharmacological treatments still have limited success ${ }^{2,3}$. Since all traits are at least partially influenced by our genetic makeup ${ }^{4}$, using genetic information to increase our understanding of the biological mechanisms underlying mental health problems might eventually benefit patients. The last two decades have seen an influx of studies assessing genetic variants related to a wide variety of behaviors, ranging from monogenic medical diseases to complex psychiatric disorders. Genomewide association studies (GWAS) provide an exploratory way to identify genetic variants throughout the genome that are, statistically, associated to a trait of interest. The explosion of GWAS studies since 2005 (https://www.ebi.ac.uk/gwas/diagram) has drastically increased our knowledge of the biology of diseases and identified thousands of variants involved in a wide variety of (disease) traits.

Yet for many complex traits, like psychiatric disorders, the identified genetic variants explain only a fraction of the variance in the trait ${ }^{5,6}$. We argue that this may, in part, be the result of the way in which neuropsychiatric traits are operationalized in genetic studies. Typically, participants are classified as cases (i.e., people that suffer from a given psychiatric disorder) or as controls (i.e., not suffering from that particular disorder). However, people suffering from the same disorder may exhibit different sets of symptoms that may, in turn, be influenced by different genetic variants. In other words, the manner in which phenotypes are operationalized will have consequences for the success of genetic analyses. Therefore, in order to properly study the genetic basis of complex behavior, it is vital to think about the exact nature of the phenotypes used in the analysis, and the way they are operationalized. This thesis uses large-scale genetic data and state-of-the-art methods to study the merits of more detailed phenotyping in uncovering the genetics of complex neuropsychiatric traits. 


\subsection{Background}

\section{All traits are heritable}

The study of twins provides an excellent opportunity for disentangling the relative contributions of environmental versus genetic influences on the variation that can be observed in behavior. Twinstudies exploit the fact that monozygotic twins (see glossary at the end of this chapter for an explanation) are (virtually) genetically identical, whereas dizygotic twins share approximately $50 \%$ of their genetic information. The degree to which behavior is heritable can then be estimated by e.g. comparing the phenotypic similarity between mono- versus dizygotic twin pairs $^{7,8}$.

Twin-studies (and behavior genetics studies in general) have shown that essentially all traits are to some extent heritable, and thus that genetic variants must exist that influence susceptibility to, or variation in, these traits ${ }^{4,9}$. That notwithstanding, there is considerable variation in heritability $\left(h^{2}\right)$ across traits. For instance, heritability of traits in the ophthalmological $\left(h^{2}\right.$ $=0.71)$ and skeletal $\left(h^{2}=0.59\right)$ domains is estimated to be substantially higher than the heritability of traits in the social values and interactions domains $\left(h^{2}=0.31 \text { and } h^{2}=0.32 \text {, respectively }\right)^{4}$. However, for all traits included in a recent meta-analysis by Polderman et al. (2015) of virtually all published twin studies since the 1950's, heritability was $>0$, and across all traits the average contribution of genetic influences was as high as $49 \%$, i.e., on average, $49 \%$ of the variation observed in behavior is due to variation on a genetic level. Although twin-studies provide valuable information on the extent to which behavior is heritable, the design does not allow identification of individual genetic variants that are associated with a given trait. That is, these studies are not informative on which and how many genes are involved, or on the magnitude of their effects.

\section{Sequencing the human genome}

The beginning of the new millennium marked the publication of the first human genome sequence ${ }^{10}$. Until then, knowledge on the human genetic code was only fragmentary, and limited indeed. The human genome project (HGP) has, for instance, taught us that approximately $6 \%$ of the genome is under purifying selection (and can thus be assumed to be functional), whereas only about $1.5 \%$ of the genome sequence encodes proteins ${ }^{11}$, which raises the question what it is these non-coding parts of the genome do. Also, the HGP shed more light on the different classes of variation that exist in the genome (see Box 1). 


\section{Box 1. Classes of genetic variation}

The human genome consists of approximately 3 billion base pairs (nucleotides; $\mathrm{A}=$ Adenine, $\mathrm{T}=$ Thymine, $\mathrm{C}=$ Cytosine or $\mathrm{G}=$ Guanine). When comparing base pairs between two individuals, most locations will hold the same information: on average, only about $0.1 \%$ of the genome sequence differs between people. Human genetic variation can be divided into several classes. Here, we broadly distinguish between single nucleotide variants (or single nucleotide polymorphisms; SNPs; pronounce: snips) and structural variants (see Fig. 1.1).

SNPs are variations in the DNA sequence where a single nucleotide is changed (e.g., at a specific position the $\mathrm{C}$ nucleotide may occur in most individuals, but is replaced by a $\mathrm{T}$ nucleotide in a minority of people). SNPs occur approximately once in every 1,000 nucleotides, and are the most common type of genetic variation. A SNP may be virtually unique (i.e., very rare SNPs), or occur in many individuals (i.e., common SNPs).

Structural variants can be subdivided into several categories (Fig. 1.1). When one or more base pairs are observed in some genomes, but not in others this is referred to as an insertion or deletion variant (often called indels). Since the genetic code is read three nucleotides (called 'codons') at a time, an indel (when it is made up of a number of nucleotides that is not divisible by three) can change the subsequent codons. This is called a reading frame shift, and may result in an altered protein. Block substitutions occur when the variation between genomes covers a sequence of nucleotides. Inversions are variants where a certain fragment of the genome is observed in reverse order. Copy-number variants occur when sections of the genome are repeated. Copy-number variations can be quite large, possibly stretching out over $2 \mathrm{Mb}$ or more. ${ }^{12}$

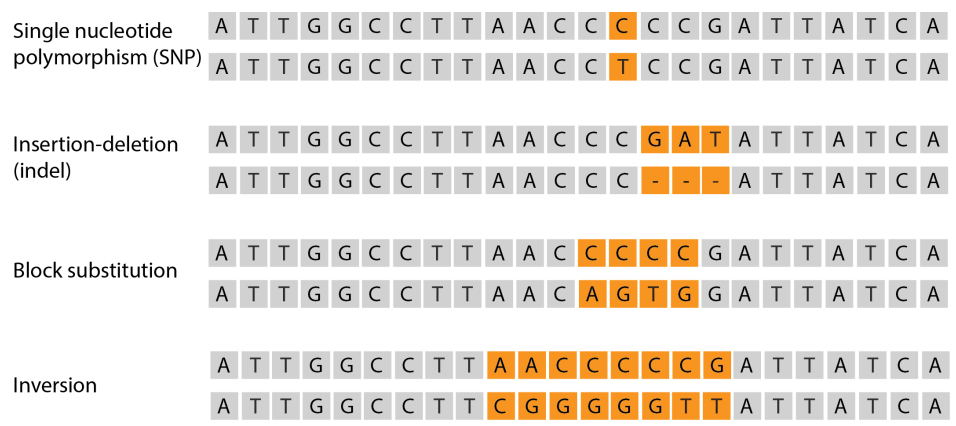

Figure 1.1. Classes of genetic variation in the human genome.

Here, the two rows represent genomes of two different individuals. This figure was inspired by Frazer et al. (2009) ${ }^{12}$. 
Another important insight was provided by the characterization of so-called linkage disequilibrium (LD) patterns throughout the genome. The mapping of $\mathrm{LD}^{13}$, regions of strongly correlated genetic variants, meant that approximately $90 \%$ of the variation in the genome can be captured by 'just a fraction' (i.e., 500,000 - 1,000,000 single nucleotide polymorphisms, or SNPs) of the total number of SNPs making up the genome ${ }^{11}$. This finding was quickly taken advantage of in the development of genotyping arrays, which now allow for measuring millions of variants simultaneously.

\section{Entering the GWAS era}

The availability of affordable tools to gather genome-wide data has made it possible to conduct large-scale studies, interrogating millions of genetic variants across the genome (genome-wide association study; GWAS; see Box 2). These techniques have enabled researchers to measure genetic variation across the genome in large samples of individuals. Unsurprisingly, the number of scientific studies into the genetic basis of human disease and behavior has seen a sharp increase since (https://www.ebi.ac.uk/gwas/diagram) ${ }^{14,15}$.

GWAS, which is inherently an exploratory approach, is particularly relevant for research into the genetics of complex traits (such as psychiatric disorders, but also non-disease behavior such as height, personality and intelligence). The reason for this is the lack of solid, a priori hypotheses on how many genetic variants are associated, and where those are located. Earlier methods, like candidate-gene and linkage studies, focused on specific, or very extensive, genomic regions or genes. Although these studies identified several genes associated to complex traits, these often proved non-reproducible later on ${ }^{16-20}$. Note however that the same is true for some initial GWAS findings that were based on too small sample sizes ${ }^{21-23}$. 


\section{Box 2. How does GWAS work?}

Genome-wide association studies (GWAS) aim to identify relationships between genetic variants (typically SNPs) and phenotypes (e.g., major depression, educational attainment, body mass index). Since the first GWAS showed that individual effects of genetic variants associated to complex traits are generally very small, large sample sizes are required to robustly detect them. If a genetic variant is associated to a disorder, say depression, this means that it will be found more (or less) often in depressed people (cases) than in healthy people (controls). In other words; the allele frequency of the SNP is expected to differ between cases and controls. For example, if the G allele is associated with a higher likelihood of being depressed, we expect to find more of these alleles in cases compared to controls (see Fig. 1.2a).

To be able to conduct GWAS we require genetic as well as phenotypic data (e.g., clinical diagnoses or responses to a questionnaire) from a large group of people. Nowadays, GWAS often includes over 100,000 people, and tests the association of a trait score to millions of genetic variants. For all these variants a statistical test of association is performed. The resulting $P$ value informs us on whether or not that specific variant is statistically associated to the trait of interest. So, essentially, millions of separate tests are performed, using dedicated software that can handle large amounts of data. With every test we perform, we accept a 5\% probability of drawing the (erroneous) conclusion that a variant is associated, while it is in fact not (false positive, or Type I error). To ensure that on the study level this probability does not exceed $5 \%$, we need to correct for the amount of independent test we perform (e.g., using the Bonferroni correction). Therefore, the standard $P$ value threshold applied in GWAS is not 0.05 , but 0.05 divided by 1 million $\left(=5 \times 10^{-8}\right)$, assuming approximately 1 million independent regions across the genome are tested (as many SNPs are not independent due to LD, we don't have to divide 0.05 by the number of SNPs we test, which will often be substantially higher than 1 million $)^{24,25}$.

GWAS results are typically visualized in a so-called Manhattan plot, in which each SNP is represented by a dot (see Fig. 1.2b). The $x$-axis indicates the genomic position (i.e., on which chromosome is the SNP located) while the $y$-axis shows the negative $\log _{10}$-transformed $P$ value (so that SNPs with a lower $P$ value are displayed at the top of the figure). All SNPs above the dashed line $\left(P<5 \times 10^{-8}\right.$; in orange) are considered genome-wide significant. 


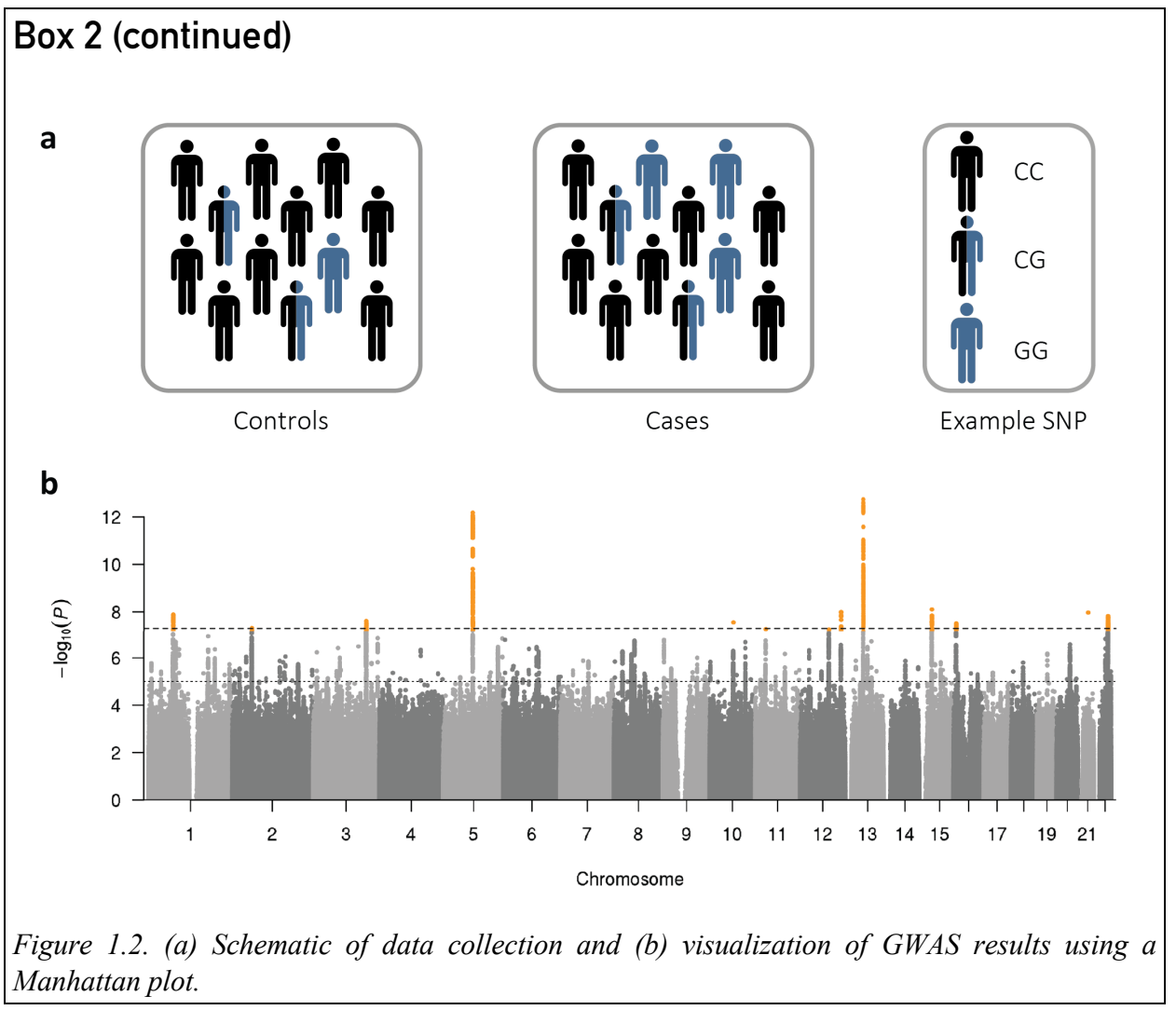

\subsection{Genetic research into psychological traits}

\section{Progress and problems of psychiatric genetic research over the last decade}

It is important to make a distinction here between studying the genetics of complex, psychological (and psychiatric) traits and (rare) medical diseases. This difference is in essence mainly due to differences in genetic architecture. Whereas some traits are monogenic (also called Mendelian) or oligogenic (i.e., variation in the trait can be explained by a single, or only a few genes), psychological traits are generally thought to be polygenic, meaning that they are influenced by a large number of genetic variants with mostly (very) small effects. A classic example of a trait that is largely caused by a single gene is cystic fibrosis ${ }^{26}$. This disease causes obstructions in the airways of the lungs and in the pancreas, leading to inflammation, tissue damage and 
destruction of both organ systems, and results in limited life expectancy ${ }^{27,28}$. The most common cause of cystic fibrosis was found to be a variant in the cystic fibrosis transmembrane conductance regulator $(C F T R)$ gene ${ }^{26,27}$. In recent years, this knowledge has led to improved screening and even the development of molecular therapy to treat the disease ${ }^{27}$.

However, for complex traits, such as psychiatric disorders (e.g. schizophrenia, depression, ADHD) and psychological traits (e.g. personality, intelligence), a different scientific trajectory is to be expected. Despite the fact that many of these traits are at least moderately heritable ${ }^{4,29-33}$ (see Fig. 1.3), the identification of associated individual genetic variants has been arduous. With the possible exception of schizophrenia ${ }^{34}$, few to none genetic variants associated to psychiatric disorders had been identified up till $2015^{6}$. More recently, collaborative efforts and large-scale biobanks, such as the Psychiatric Genomics Consortium (PGC) and the UK Biobank (UKB) ${ }^{35,36}$, have resulted in modest success. Even for disorders like depression and ADHD, for which identification of genetic variants has proven particularly challenging ${ }^{37}$, the first discoveries have now been reported ${ }^{38-40}$. These and other studies have thus shown us that, presumably for any trait, increasing sample size is likely to result in eventually identifying associated variants ${ }^{41}$.

Nonetheless, the proportion of variation in a trait that can be explained by all genetic variants across the entire genome (referred to as SNP-based heritability; $h^{2}{ }_{S N P}$ ), is typically substantially lower than the heritability estimates from twin-studies ${ }^{5,6}$ (Fig. 1.3). This gap is befittingly coined the "missing heritability' ${ }^{42-44}$ (Wray et al. (2014) distinguish missing, hidden and still-missing heritability). Even using all millions of SNPs included in modern-day GWAS, it's not possible to explain more than just a (small) part of the variation in a given trait.

Several possible explanations have been brought forward for the missing heritability problem ${ }^{43}$. For instance, genotyping arrays are not well suited for detecting rare variants, although these might have large effects ${ }^{45,46}$. The availability of large-scale whole exome or whole genome sequencing data (i.e., mapping the entire genomic sequence rather than a selection of (common) SNPs like in GWAS) in the near future might help determine the relative impact of rare variants on complex traits $^{47,48}$. A recent study ${ }^{49}$ already reported that the full heritability for height and BMI could be recovered from whole-genome sequence data. However, for psychological traits such progress is yet to be made. In addition, GWAS usually considers additive effects, ignoring potential dominance or gene by gene interaction effects ${ }^{50}$. Notwithstanding these caveats, there is little evidence that gene by gene interactions (or interactions between SNPs; epistasis) contribute substantially to the variance in traits ${ }^{41,51}$. 


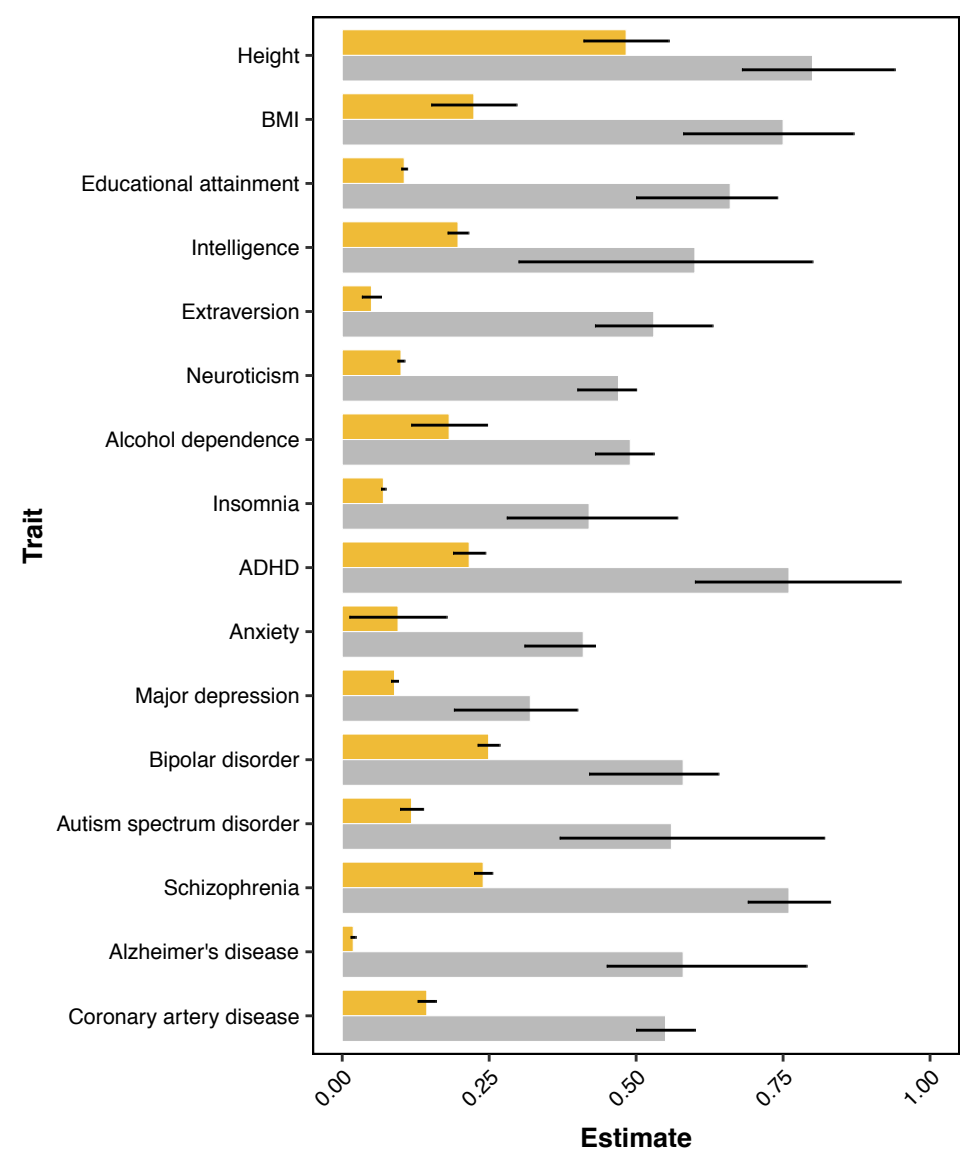

Figure 1.3. Heritability estimates for a selection of complex traits.

Heritability estimates derived from twin studies (in gray) $)^{6,32,52-66}$ and computed based on all

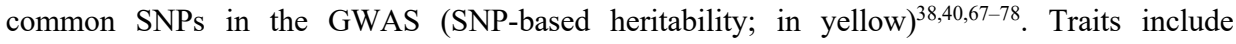
anthropometric traits (e.g., height), psychological constructs (e.g., intelligence and neuroticism), psychiatric disorders (e.g., ADHD and major depression) and traits in the medical domain (e.g., coronary artery disease). Note that the estimates of the twin heritability should be interpreted as an indication rather than as exact estimates. For convenience we collected the SNP-based heritability estimates through GWAS ATLAS ${ }^{79}$, hence values may deviate slightly from what was reported in the original paper. For the twin heritability estimates the error bars represent $95 \%$ confidence intervals where possible (if 95\% confidence intervals were not reported we used lower and upper bounds of the range of estimates for that trait). For the SNP-based heritability estimates the error bars represent standard deviations (i.e., $1.96 \times \mathrm{SE}$ ). 
Lastly, the missing heritability problem is often thought to result from the large number of genetic variants affecting complex traits (i.e., polygenicity), with each single variant having only a (very) small effect ${ }^{80,81}$ (this model is referred to as the infinitesimal model ${ }^{82}$, which states that all variants have non-zero, but very small, effects). Reliably estimating these tiny effects requires high statistical power, and hence, very large samples. As the recent focus of (psychiatric) genetics has very much been on increasing sample sizes, one might conclude this is the most acted-upon explanation for the missing heritability, and the limited findings of GWAS. While all these explanations are likely and valid, they all relate to genetic issues. However, GWAS analyses do not only rely on genetic, but also on phenotypic information. The research in this thesis focusses on how modelling the phenotype may affect the success of genetic enterprises.

\section{Phenotypic complexity; shining a light on phenotypic issues}

Rather than in shortcomings in the genetic information (e.g., focus on common variants) or model (i.e., additive effects), explanation for the limited findings from genetic studies of psychological traits could also be sought in the phenotypic information used in genetic analyses. Although the missing heritability cannot be solved by better phenotyping (since $h^{2}$ and $h^{2}{ }_{S N P}$ are using the same phenotypic operationalization), one could argue that it would be reduced in more detailed, homogeneous phenotypes, that have more direct links to e.g. genetic pathways ${ }^{83}$. Focusing on psychological and psychiatric traits, the definition of phenotypes is not necessarily straightforward. For example, a trait may actually be an aggregation of subtypes, instead of a single, clearly characterized, disease entity ${ }^{84,85}$. So far, there has been relatively little attention for the question of how to measure and model phenotypes, despite the fact that the answer to this question may affect the potential success of genetic studies ${ }^{85}$, as we will discuss below.

Psychological traits are generally operationalized using items on a questionnaire ${ }^{86-88}$, or as the (dichotomized) sum of symptoms (e.g., sum-score or case-control status), whose information is obtained in a clinical interview (i.e., the "gold standard", see Kapur et al., 2012 ${ }^{89}$ ). Combining symptoms of a test, (diagnostic) questionnaire, or clinical interview can be convenient, for instance, if the goal is to obtain a general indication of a subject's intelligence, personality, or psychopathology. Such a score can then be used as predictor, or to inform treatment. However, while such an approach arguably has predictive and clinical utility ${ }^{90}$, its usefulness for scientific research into the biological mechanisms underlying traits may be limited (i.e., it is conceivable that symptoms have different genetic determinants, and summing these symptoms may dilute their genetic signal).

In genetic studies, summing the symptoms underlying a trait reflects the implicit assumptions that all symptoms are 1) equally good indicators of the trait in question, and 2) are influenced by the same set of genes (i.e., are genetically homogeneous). The model that fits these criteria is referred to as the reflective (latent variable) model (i.e., all symptoms are thought to be caused by one underlying latent, unobservable trait) ${ }^{91}$. Taking major depression as an example; what we 
generally observe is a number of symptoms (e.g., as defined in the Diagnostic and Statistical Manual of mental disorders; DSM $^{92}$ ), such as worrying, sleeping and concentration problems, fatigue, and feelings of guilt. According to the reflective model, the co-occurrence of these symptoms is explained by their common cause; the latent trait major depression, which cannot be directly observed or quantified itself (Fig. 1.4a). If there is indeed a latent trait, depression, that causes these observable symptoms, a GWAS on the sum-score would be suitable to identify associated genetic variants, as all genetic effects act through the latent variable ${ }^{85}$. Unfortunately, we generally do not know the true trait-generating model.

Alternative models have been proposed that are equally capable of explaining the relationships between observed symptoms, but would call for a different approach in genetic research. The first model, which we will refer to as the formative model (Fig. 1.4b), bears resemblance to the previously discussed reflective model ${ }^{91}$. The critical distinction is that according to the reflective model, the latent variable causes the symptoms, whereas according to the formative model it is merely an index variable of those symptoms, i.e., a convenient summary of their concerted effects. In other words, the direction of causation is reversed.

If the formative model is the true trait-generating model, then this would allow for genetic effects on individual symptoms, rather than all genetic effects acting on the latent level. In such cases, testing for association between genetic variants and a sum-score is suboptimal, as e.g. the signal of variants uniquely affecting a single symptom will be severely diluted. The notion that at least not all symptoms are created equal, i.e., not all symptoms are equally informative indicators of the latent/index variable, is supported by research showing that MD symptoms differ in their relations to a variety of clinical validators, such as duration of the depressive episode and the odds of developing substance dependence ${ }^{93}$.

Another alternative model, that has become increasingly popular in the field of psychology over the last decade, is the network model ${ }^{94-96}$. In contrast to the reflective model, a network conceptualization of a trait obviates the need for a latent variable. Rather, the co-occurrence of symptoms is explained by direct, mutual, possibly causal relations between symptoms (Fig. 1.4c). For example, returning to the MD example, it is conceivable that worrying causes sleeplessness which in turn affects one's ability to concentrate, which may then lead to subsequent feelings of guilt for not functioning properly. Thus, in this framework, the symptoms influence, maintain, and reinforce each other, collectively giving rise to what we refer to as depression ${ }^{97,98}$. 
a

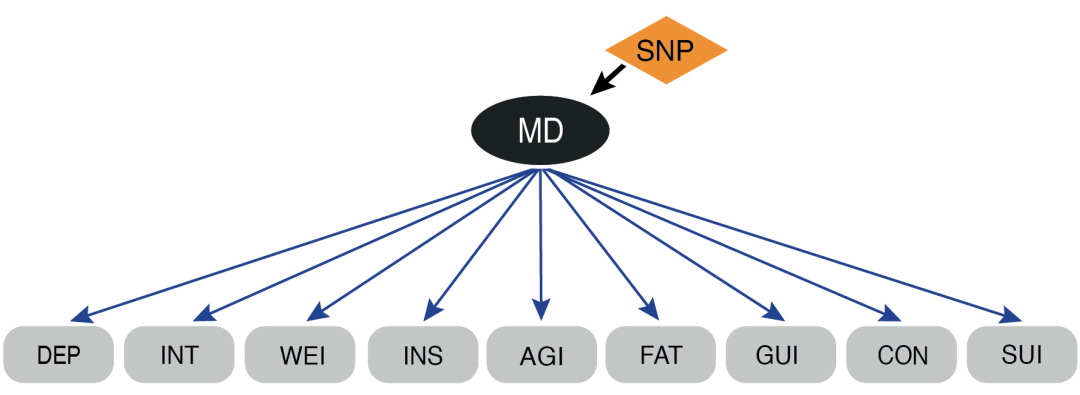

b

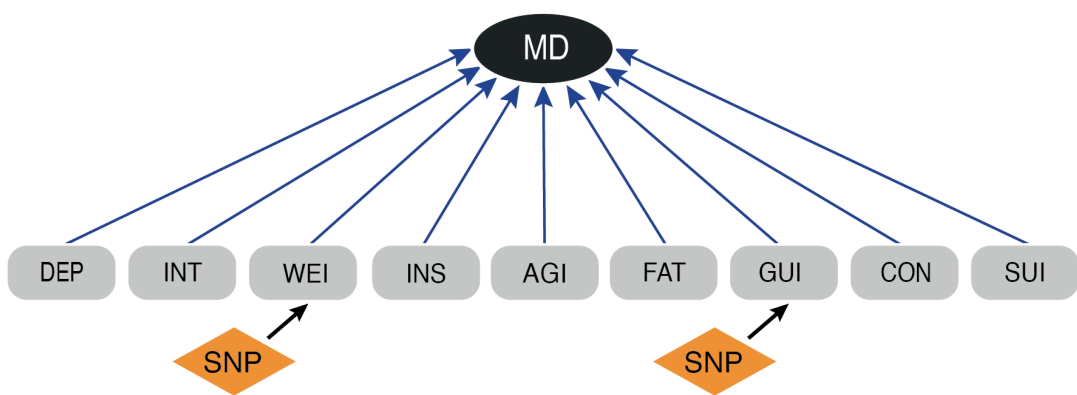

C

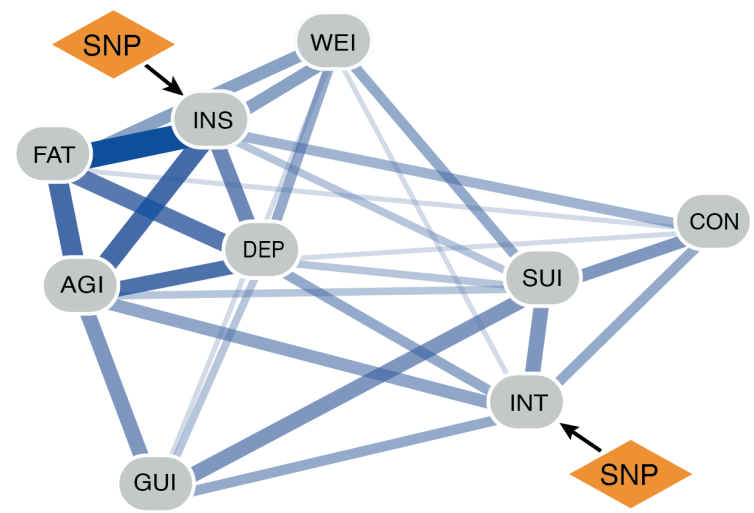

Figure 1.4. Models to conceptualize depression

Major depression (MD) conceptualized as (a) a reflective latent variable model where all genetic effects are implicitly assumed to affect the symptoms through the latent variable (MD), (b) a formative latent variable model, where the latent variable MD is regarded as an index variable of the symptoms, and (c) a network model in which symptoms are thought to directly affect each other. The latter two models are compatible with genetic variants (SNPs) influencing individual symptoms. The width and hue of the edges in the network indicate the strength of correlation between two symptoms. $\mathrm{MD}=$ major depression; $\mathrm{DEP}=$ depressed mood; INT=loss of interest; WEI=weight loss/gain; INS=insomnia; AGI=psychomotor agitation; FAT=fatigue; GUI=feelings of guilt; $\mathrm{CON}=$ concentration problems; $\mathrm{SUI}=$ suicidal thoughts. 
From a network perspective, items or symptoms are not measures of a disorder, but the network of symptoms or items in itself constitutes the disorder. Consequently, there is no reason to assume genetic variants to affect all symptoms; genetic variants might act on some individual items or symptoms, but not, or not in the same way, on others. In practice, analyzing the sum-score calculated across all items or symptoms, or a dichotomized case-control version hereof, is then likely to identify genetic variants that are 'global' (i.e., affect all or most symptoms), but miss 'local' variants (i.e., genetic variants that affect one or more, but not all, items or symptoms) ${ }^{85}$.

In contrast to reflective and formative models, a network perspective of psychopathology naturally facilitates complexity, and can explain various psychological phenomena. This is nicely illustrated by the wide range of applications within the field of psychology. For instance, Cramer et al. $(2010)^{99}$ used a network approach to explain comorbidity between MD and generalized anxiety disorder. They found strong evidence for comorbidity, as the network included some strong relations across, rather than within, symptoms of traits. Moreover, they show that the network view of comorbidity accommodates for sensible hypotheses on overlapping symptoms and pathways to comorbidity, that are not reconcilable with a latent trait model. Other studies have applied the network model in a clinical context. Addressing the dynamics of depression and post-traumatic stress disorder (PTSD) showed which symptoms are most likely to influence the symptom networks of these traits, and hence, are most interesting as treatment targets ${ }^{100,101}$. Another study reported that the structure of the symptom network at an earlier stage was predictive of the course of depression over time ${ }^{102}$.

To summarize, currently, GWAS of psychological traits relies heavily on the reflective model. Essentially, by adopting a reflective model "we have taken an index of something for the thing itself" (Kendler, 2016) ${ }^{103}$. This despite the fact that there is little empirical support for this specific model. However, the theoretical notion of the model underlying psychological traits has implications for what can be expected from these analyses, as misspecification may introduce noise (i.e., unexplained variance) in the analysis. We argue that the unilateral focus on this potentially incorrect model, might be part of the reason for the relatively disappointing yield from genetic studies into psychological traits.

Analyzing a sum-score is only the optimal approach if all (or most) symptoms are affected by the same genetic variants. On the other hand, if symptoms are affected by different genetic variants (i.e., when traits are generated through a formative model or network model), statistical power is likely to decrease when analyzing a sum-score ${ }^{85}$. Therefore, the study of genetic determinants of psychological traits might benefit from exploring these alternative phenotypic models. 


\section{Sources of phenotypic heterogeneity}

As described in the previous section, misspecification or misconceptualization of the traitgenerating genotype-phenotype model is one source of noise that leads to diluted genetic effects. However, there are other factors that may introduce noise in the analysis. Since there exists no single, universally objective method to measure psychological and psychiatric traits, reliable and accurate classification of disease entities is impossible. Many of the problems arising on the phenotypic side, boil down to the concept of phenotypic heterogeneity. Phenotypic heterogeneity means that a trait does in fact not represent a single, homogeneous construct. For example, individuals diagnosed with MD may exhibit very different symptom profiles. For instance, patient A may experience loss of interest, decreased appetite, hypersomnia, excessive feelings of guilt and inability to concentrate, while patient B experiences irritable mood, increased appetite, insomnia, feelings of worthlessness and suicidal ideation. Despite these differences, both symptom patterns result in an MD diagnosis, and MD can therefore be said to be a phenotypically heterogeneous disorder. Yet in genetic studies, all patients with the same diagnosis are classified as 'cases' under the assumption that people with the same diagnosis are genetically similar. As mentioned before, such overall diagnoses may serve a clinical purpose, but might be unsuitable for studying the genetic roots of psychological traits. Phenotypic heterogeneity can result in underestimation of the $h^{2}{ }_{S N P}$ and markedly decreased power of GWAS ${ }^{44}$. Here we discuss a number of inter-related factors that can contribute to phenotypic heterogeneity.

Firstly, comorbidity, in which an individual is diagnosed with multiple disorders, is widespread $^{1,104,105}$. Comorbidity may arise from fuzzy symptomological borders, resulting in multiple diagnoses applying to the same person ${ }^{104,106}$, or from genuine shared underlying biological mechanisms across disorders. For example, diagnostic questionnaires frequently used for MD and anxiety both include items on feeling restless, worrying, and nervousness. Recent studies have shown substantial genetic overlap between these disorders, i.e., comorbidity, on the genetic level ${ }^{107,108}$. Whether this comorbidity and the genetic overlap are due to the overlap in the way these disorders are measured, or rather truly point to shared biology of two distinct diagnostic constructs, merits further research.

A second, related, concept resulting in phenotypic heterogeneity is misdiagnosis. In misdiagnosis, different (or the same) clinicians arrive at different diagnoses within the same person over time ${ }^{109,110}$. Analogous to comorbidity, misdiagnosis may be the consequence of vague, imprecise diagnostic criteria. Alternatively, a disorder may actually develop into another, or remit, over time, creating a discrepancy between diagnoses at different time points. Whereas with comorbidity multiple diagnoses may actually apply to a person at the same time, misdiagnosis suggests an incorrect assessment. In practice, misdiagnosis and comorbidity may be hard, if not impossible, to distinguish.

Both problems discussed above are potential consequences of the fact that the phenotypes used in genetic studies are typically composite measures, e.g. a (dichotomized) sum-score of symptoms from a diagnostic interview. In many disorders, patients with the same diagnosis can have different 
symptom profiles ${ }^{111,112}$. These individual symptoms may have different risk factors and impact on impairment ${ }^{93,113,114}$. For instance, a study assessing the effects of individual depression symptoms on impairment in several domains (e.g., work, close relationships, social activities) found that sad mood and concentration problems were substantially stronger predictors of impairment compared to other symptoms ${ }^{113}$. Another study reported risk factors that are associated to all symptoms (e.g., neuroticism and work hours), but also risk factors that are uniquely associated to single (or subsets of) symptoms (e.g., stressful life events are associated to concentration problems and suicidal ideation), or specific to males or females ${ }^{114}$. In addition, diagnostic criteria may include dualistic symptoms (e.g., for major depression: insomnia or hypersomnia, appetite/weight loss or appetite/weight gain). When simply using the number of endorsed symptoms in an analysis, valuable information about both the nature and severity of the individual symptoms, and as a result the disease as a whole, is lost. A possible solution to this problem would be to acknowledge the phenotypic heterogeneity, and start studying the genetics of individual symptoms rather than their composite sum-scores.

\section{Summary}

To conclude, psychology and psychiatry are not exact sciences, and mental disorders aren't entities that can be assessed in a purely objective manner. The diagnostic boundaries that may be useful from a clinical perspective, are in fact not as clear as we would like them to be. People suffering from mental disorders often qualify for multiple diagnoses, and people with the same disorder may indeed manifest very diverse symptoms. Moreover, many traits, e.g. MD, anxiety, neuroticism, share a genetic background ${ }^{71,107,115}$. On the one hand this might reflect actual shared biological mechanisms, on the other it may be a consequence of overlapping diagnostic criteria (i.e., shared symptoms used to assess these traits). In other words; the observed genetic correlations between psychiatric traits ${ }^{107,116-118}$ may reflect genuine, shared etiology, or may be a consequence of the operationalization of traits used in genetic analysis (or a combination thereof). As Kapur et al. (2012) put it "The current diagnostic system was not designed to facilitate biological differentiation and it does not".

All issues discussed here decrease the extent to which traits are unidimensional, homogeneous entities, in other words; they result in phenotypic heterogeneity. Whether such phenotypic heterogeneity indeed reflects genetic heterogeneity is unknown, but the reality of phenotypic heterogeneity does beg the question how realistic it is to expect clearly defined biological pathways emerging from genetic analysis of psychological traits (a related discussion was initiated by Turkheimer $\left(2000^{9}, 2016^{119}\right)$ who stated that for various reasons interpretable genetic or biological mechanisms may not always be identifiable for traits with non-zero heritability, distinguishing "strong" versus "weak" genetic explanation). A growing body of research is committed to studying this question. Results on traits like major depression, bipolar disorder and schizophrenia ${ }^{76,120-123}$ suggest that the field of psychiatric genetics may benefit from 
more detailed phenotypic definitions ${ }^{85,124,125}$. In this thesis we specifically attempt to formulate an answer to this question.

\subsection{Aims and outline of this thesis}

\section{Approaches to improve gain from genetic studies of psychological traits}

This thesis aims to shed more light on the question whether traits conceptualized at the phenotypic level are also discernible on the genetic level. Is it reasonable to assume a one to one relationship between the phenotypic and genetic level, when it comes to psychological traits?

Over the last decade the field of complex trait genetics has dedicated itself to increasing study population sample sizes, thereby increasing the likelihood (i.e., statistical power) of identifying small-effect genetic variants that are statistically associated to the trait in question. As discussed earlier, this approach has substantially increased the number of hits for many traits ${ }^{41}$. However, reducing phenotypic heterogeneity in psychiatric genetic studies may also greatly impact power ${ }^{85,124,126}$. One possible way to do this is to study individual items or symptoms, rather than a sum-score or case-control status. The use of individual symptoms, or homogeneous subtypes of a trait, has the additional advantage that genes or biological pathways associated to these phenotypes may show higher molecular coherence and trait specificity ${ }^{127}$, and as such, may be easier linked to underlying biological mechanisms ${ }^{83}$.

We note that both approaches (increasing sample size \& reducing phenotypic heterogeneity) could in principle complement each other, and should not be regarded as conflicting methods. Unfortunately, very few large-scale data collections with symptom-level phenotypic information are currently available. In fact, this is the one practical issue that has impeded the current line of research. Combining different sources of data, even when item-level data is available in the first place, is often problematic due to the use of different measurements (i.e., different questionnaires may have been used) across different samples. Although some have used item-response theory (IRT) to harmonize trait-level scores across different measurement instruments ${ }^{128}$, this obstructs the aggregation of data on the symptom-level. However, initiatives like the UK Biobank, or consortia established with the specific aim of collecting data on a trait of interest using similar data acquisition protocols, may aid in providing the research community with high-quality phenotype data.

\section{Outline of this thesis}

Chapter 2. Item-level analyses reveal genetic heterogeneity in neuroticism

Similar to the general approach in the field, previous research on the genetics of neuroticism ${ }^{129-132}$ typically used an aggregate measure, combining information from multiple different neuroticism 
items (e.g. a sum-score). This reflects the assumption that the items are genetically similar. In this first chapter, we aimed to put this assumption to the test.

Hereto, we separately analyzed 12 neuroticism items of the Eysenck Personality Questionnaire $(\mathrm{EPQ})^{88}$ in over 366,000 people in the UK Biobank. Neuroticism is a heritable personality trait $\left(h^{2}\right.$ $\sim 0.45)^{4,33}$, and individuals that score high on this trait tend to experience negative affect and are sensitive to negative stimuli. Neuroticism is a stable personality trait that correlates with psychiatric traits like anxiety, substance abuse, and $\mathrm{MD}^{115,133,134}$, and is one of the few phenotypes for which large-scale item- or symptom-level data are available.

After conducting GWAS on the 12 separate items, we computed genetic correlations between all the items, and replicated the correlational structure in a second dataset. Given the assumption of genetic homogeneity of items or symptoms, one would expect very high genetic correlations $\left(r_{g} \sim\right.$ 1) among the items, yet genetic correlations between the items ranged between 0.38 and 0.91 . Subsequently, we applied clustering analysis to the genetic correlations to determine whether homogeneous clusters of items could be identified within the total pool of neuroticism items. Finally, a wide range of analyses was performed (including genetic correlations, functional annotation, gene-set analysis) to describe and compare the genetic signal between the individual items, and relate it to results for the overall sum-score.

Chapter 3. Meta-analysis of genome-wide association studies for neuroticism in 449,484 individuals identifies novel genetic loci and pathways

Whereas in Chapter 2 our main focus was to assess the genetic homogeneity of the neuroticism personality trait, the goal of the second chapter was to provide a more in-depth analysis of the genetic determinants for neuroticism, its subclusters, and their mutual relation. Specifically, we aimed to further specify neuroticism's neurobiological interpretation by comparing the genetic signal of the full neuroticism trait to that of two genetically distinguishable neuroticism subclusters, 'depressed affect' and 'worry'. As a validation of the depressed affect dimension, we also compared the genetic signal of neuroticism and the two subclusters to that of depression.

In order to maximize the probability of finding associated genetic variants, we combined the UK Biobank data with data of two other cohorts, resulting in a total sample size of almost 450,000 participants. In addition, we conducted second meta-analysis of depressive symptoms, which is strongly correlated with MD (phenotypic correlation $r=0.58$, genetic correlation $r_{g}=0.68$ ) in > 688,000 people. We conducted a wide range of analyses, including cross-trait genetic correlations, gene-set analysis, and functional annotation analysis to assess the multidimensionality of neuroticism and its link to depression.

Chapter 4. Examining the biological validity of internalizing disorders through genetic item-level analyses

Chapter 4 revolves around the question whether the traits that are defined on a phenotypic level (e.g., major depression, anxiety disorder) are also distinguishable on the genetic level. We 
specifically aimed to evaluate whether an item-level approach can improve our understanding of the genetic etiology of internalizing disorders (MD and anxiety disorders; ADs).

We used factor analysis to empirically identify the genetic and phenotypic structure of items, and validated the results with multiple genetic association approaches. The focus on internalizing disorders stems from the fact that phenotypic definitions are particularly challenging within this spectrum due to overlapping diagnostic criteria, massive cross-disorder genetic correlation ${ }^{135-137}$. The aims of the current chapter were twofold: 1) to identify genetically homogenous clusters of symptoms and 2) to compare the genetically derived clusters of symptoms with the theoretically derived clusters (i.e., clinical scale sum scores, corresponding to the traditional classification), through e.g. comparison of their genetic correlations with external phenotypes.

Chapter 5. Genome-wide meta-analysis of brain volume on 47,316 individuals identifies genomic loci and genes shared with intelligence

Brain volume and intelligence are correlated in humans $(r \approx 0.40)$, and twin studies showed that this correlation is fully due to shared genetic factors ${ }^{138}$. The availability of genomic data now allows for examining the genetic overlap between these two traits on the genetic level, enabling us to establish whether there is evidence of shared biological pathways and mechanisms.

We first conducted a meta-analysis of brain volume, using fMRI data as well as measures of head circumference in children (which has proven to be a good proxy for brain volume), resulting in the largest GWAS analysis of brain volume at the time of writing $(N=47,316)$. In a subsequent series of analyses, we then focused on dissecting the genetic overlap between brain volume and intelligence, by studying similarities and differences in associated genomic loci, likely causal genes, associated cell types and gene expression profiles throughout the brain. 


\subsection{Glossary of important concepts, as used throughout this thesis}

\begin{tabular}{|c|c|}
\hline Word & Meaning \\
\hline Base pair & $\begin{array}{l}\text { Two DNA bases that are complementary to one another }(A / T, C / G) \text {, and link with } \\
\text { other base pairs to form the typical double helix structure of DNA. }\end{array}$ \\
\hline $\begin{array}{l}\text { Bonferroni } \\
\text { correction }\end{array}$ & $\begin{array}{l}\text { A method to correct statistical outcomes for multiple comparisons. When } \\
\text { performing multiple tests, the likelihood of incorrectly concluding that there is an } \\
\text { effect (i.e., rejecting the null hypothesis) increases. To guard against this } \\
\text { phenomenon, one can compensate by testing each hypothesis at a significance } \\
\text { level of } 0.05 \text { (e.g., when conducting } 10 \text { tests, the Bonferroni-corrected } \\
\text { significance threshold would be: } 0.05 / 10=0.005 \text { ). }\end{array}$ \\
\hline $\begin{array}{l}\text { Broad-sense } \\
\text { heritability }\end{array}$ & $\begin{array}{l}\text { Proportion of heritability that is due to all genetic factors (including dominant \& } \\
\text { recessive factors and interactions between genetic factors). See: Heritability }\end{array}$ \\
\hline Complex trait & $\begin{array}{l}\text { Complex traits are typically caused by a multitude of genetic variants, } \\
\text { environmental factors, and their interactions (see Polygenic). Psychiatric traits } \\
\text { generally fit the definition of complex traits. }\end{array}$ \\
\hline Dizygotic twin & Fraternal twins. Developed from separate eggs, fertilized by separate sperm cells. \\
\hline Gene-based analysis & $\begin{array}{l}\text { Gene-based analysis evaluates the joint association effect of all SNPs within a } \\
\text { gene yielding a gene-based } P \text { value. This may result in significant association of a } \\
\text { gene despite all SNPs within that gene not reaching the genome-wide significance } \\
\text { threshold (or vice versa; a gene not being significantly associated while one or } \\
\text { more SNPs are). }\end{array}$ \\
\hline Gene-set analysis & $\begin{array}{l}\text { Gene set analysis assesses whether the genes associated to a trait of interest are } \\
\text { enriched in pre-specified gene sets. Gene sets can be constructed based different } \\
\text { criteria, e.g., expert consensus, known function etc.). }\end{array}$ \\
\hline Genetic architecture & $\begin{array}{l}\text { A combination of the number of variants influencing a phenotype, the magnitude } \\
\text { of their effects on this phenotype, their frequency in the population and their } \\
\text { interactions with each other and the environment. }\end{array}$ \\
\hline $\begin{array}{l}\text { Genetic } \\
\text { heterogeneity }\end{array}$ & $\begin{array}{l}\text { The phenomenon where one phenotype (or very similar phenotypes) are the } \\
\text { result of different genetic mechanisms. In a more practical sense; a trait may be } \\
\text { considered genetically heterogeneous when multiple (sets of) genetic risk } \\
\text { variants exist in the population that all lead to a similar phenotype (in other } \\
\text { words; when clusters of individuals exist that exhibit the same trait but carry } \\
\text { different (combinations of) risk alleles in a common set of SNPs) } \\
\text { Alternatively; a composite phenotype (e.g., a sum-score), created by combining } \\
\text { genetically different symptoms, may be considered genetically heterogeneous as } \\
\text { the individual symptoms may be associated to different (sets of) genetic risk } \\
\text { variants. }\end{array}$ \\
\hline
\end{tabular}




\begin{tabular}{|c|c|}
\hline Word & Meaning \\
\hline Genetic variant & $\begin{array}{l}\text { There are several types of genetic variation. Here we typically mean a SNP (see: } \\
\text { Single nucleotide polymorphism) }\end{array}$ \\
\hline Genome & $\begin{array}{l}\text { The genome is an individual's complete set of DNA (in other words, the entire } \\
\text { genetic sequence, containing all of its genes). The human genome consists of } \\
\text { about } 3 \text { billion DNA base pairs. }\end{array}$ \\
\hline $\begin{array}{l}\text { Genome-wide } \\
\text { association study } \\
\text { (GWAS) }\end{array}$ & $\begin{array}{l}\text { Study that tests for statistical association of all measured genetic variants } \\
\text { (typically SNPs) with a trait or disorder of interest. See Box } 2 \text { for a more detailed } \\
\text { description of GWAS. }\end{array}$ \\
\hline Genotype & $\begin{array}{l}\text { The two alleles in an individual on a particular genetic variant. Also used as a verb } \\
\text { (genotyped) to refer to the process of determining differences in the genetic } \\
\text { information carried by an individual. }\end{array}$ \\
\hline Heritability & $\begin{array}{l}\text { Proportion of the total variance in a trait that can be explained by genetic } \\
\text { variation between individuals in the population. Note that heritability does not } \\
\text { place an upper bound on the influence of environment (and as such, does not } \\
\text { suggest genetic determinism). Heritability is merely an indication of the relative } \\
\text { contribution of genetic factors to a trait, as measured in a specific population. This } \\
\text { means that heritability estimates may vary across populations. For example; } \\
\text { consider educational attainment. This trait is obviously partly driven by } \\
\text { accessibility of schooling. Since most children in the Netherlands have the } \\
\text { opportunity to go to school, unlike in some developing countries, the variation in } \\
\text { environmental factors influencing educational attainment is smaller than in } \\
\text { developing countries. Therefore, the relative contribution of genetic factors to } \\
\text { differences in educational attainment (i.e., heritability) between people will be } \\
\text { larger. }\end{array}$ \\
\hline $\begin{array}{l}\text { Linkage } \\
\text { disequilibrium (LD) }\end{array}$ & $\begin{array}{l}\text { Genetic variants that are situated closely to each other tend to be associated in a } \\
\text { non-random way. If two genetic variants (or regions) are in linkage equilibrium } \\
\text { that means they are inherited in a completely independent fashion. Conversely, } \\
\text { linkage disequilibrium indicates that two genetic variants are not inherited } \\
\text { independently. }\end{array}$ \\
\hline Manhattan plot & $\begin{array}{l}\text { Plot that is often used to visualize the results of GWAS. On the } x \text {-axis the genomic } \\
\text { position (typically indicated by chromosome number) is shown, while the } y \text {-axis } \\
\text { features the } P \text { value of association (usually negative log10-transformed, so that } \\
\text { SNPs that have a lower } P \text { value are positioned higher). Each dot in the plot } \\
\text { represents a SNP. This type of figure was coined Manhattan plot due to its } \\
\text { similarity to the skyline of Manhattan (that is, if there is significant genetic signal!). }\end{array}$ \\
\hline Mendelian trait & $\begin{array}{l}\text { Traits that follow a Mendelian pattern of inheritance (i.e., traits that are } \\
\text { determined by variation in a single genetic variant). }\end{array}$ \\
\hline $\begin{array}{l}\text { Minor allele } \\
\text { frequency }\end{array}$ & $\begin{array}{l}\text { The frequency of the less frequent allele at a genetic variant in the population. } \\
\text { Very rare genetic variants require specialized analysis methods, since there are } \\
\text { too few observations to apply regular (i.e., common variant) association } \\
\text { techniques. }\end{array}$ \\
\hline
\end{tabular}




\begin{tabular}{|c|c|}
\hline Word & Meaning \\
\hline Monogenic & $\begin{array}{l}\text { Monogenic traits or disorders are the result of a mutation in a single genetic } \\
\text { variant. }\end{array}$ \\
\hline Monozygotic twin & Identical twins. Developed from the same fertilized egg (=zygote). \\
\hline $\begin{array}{l}\text { Narrow-sense } \\
\text { heritability }\end{array}$ & $\begin{array}{l}\text { Proportion of heritability that is due to additive genetic factors only. See: } \\
\text { Heritability }\end{array}$ \\
\hline Oligogenic & $\begin{array}{l}\text { Oligogenic traits or disorders are the result of mutations in multiple genetic } \\
\text { variants. Oligogenic inheritance essentially falls in between monogenic and } \\
\text { polygenic inheritance. }\end{array}$ \\
\hline Phenotype & Any characteristic of an individual that can be measured (e.g., height, behavior). \\
\hline Pleiotropy & $\begin{array}{l}\text { Pleiotropy is the phenomenon where a single gene (or genetic variant) is } \\
\text { associated to multiple seemingly unrelated phenotypes. }\end{array}$ \\
\hline Polygenic & $\begin{array}{l}\text { Polygenic traits or disorders are the results of mutations in many different genetic } \\
\text { variants, often combined with environmental influences. }\end{array}$ \\
\hline $\begin{array}{l}\text { Population } \\
\text { stratification }\end{array}$ & $\begin{array}{l}\text { Allele frequencies may differ systematically between populations (possibly due to } \\
\text { different ancestry). This is referred to as population stratification. It is very } \\
\text { important to correct for potential effects of population stratification in GWAS, } \\
\text { since they are likely to bias the results and may lead to spurious associations. }\end{array}$ \\
\hline Power & $\begin{array}{l}\text { Statistical power is the probability of correctly rejecting a false null hypothesis. In } \\
\text { other words; the probability of detecting an effect when there is one. E.g., the } \\
\text { probability of detecting a SNP associated to a trait when that SNP is in fact } \\
\text { associated to that trait. }\end{array}$ \\
\hline QQ-plot & $\begin{array}{l}\text { In a quantile-quantile (QQ) plot the expected } P \text { value is plotted against the } \\
\text { observed } P \text { value. QQ plots are used in GWAS to determine whether the observed } \\
\text { SNP } P \text { values deviate (i.e., are not on the diagonal line) from what would be } \\
\text { expected if none of the SNPS was truly associated to the trait of interest. }\end{array}$ \\
\hline Quantitative trait & See: complex trait \\
\hline $\begin{array}{l}\text { SNP (Single } \\
\text { nucleotide } \\
\text { polymorphism) }\end{array}$ & $\begin{array}{l}\text { Also: Single nucleotide variant (SNV). Single base pair positions in the genome } \\
\text { where a single nucleotide in the sequence can vary (e.g. A or C). SNPs are defined } \\
\text { as being 'common', i.e., at least } 1 \% \text { of the population carries an alternative allele. }\end{array}$ \\
\hline $\begin{array}{l}\text { SNP-based } \\
\text { heritability }\left(h^{2} \text { SNP) }\right.\end{array}$ & $\begin{array}{l}\text { Proportion of the variance in a trait that can be explained by all SNPs included in } \\
\text { a GWAS. } h 2 S N P \text { can be estimated using tools like GCTA and LD score regression. }\end{array}$ \\
\hline
\end{tabular}





\section{Item-level analyses reveal genetic heterogeneity in neuroticism}

Mats Nagel ${ }^{1}$, Kyoko Watanabe ${ }^{2}$, Sven Stringer ${ }^{2}$, Danielle Posthuma ${ }^{1,2 \S}$, Sophie van der Sluis ${ }^{1 \S}$

1. Department of Clinical Genetics, Section Complex Trait Genetics, Center for Neurogenomics and Cognitive Research, Amsterdam Neuroscience, VU Medical Centre, Amsterdam $1081 \mathrm{HV}$, The Netherlands.

2. Department of Complex Trait Genetics, Center for Neurogenomics and Cognitive Research, Amsterdam Neuroscience, VU University Amsterdam, Amsterdam 1081 HV, The Netherlands.

$\S \quad$ These authors jointly supervised this work

Published in: Nature Communications (2018). Volume 9, doi:10.1038/s41467-018-03242-8 


\section{Abstract}

Genome-wide association studies (GWAS) of psychological traits are generally conducted on (dichotomized) sums of items or symptoms (e.g., case/control status), and not on the individual items or symptoms themselves. We conduct large-scale GWAS on 12 neuroticism items and observe notable and replicable variation in genetic signal between items. Within samples, genetic correlations among the items range between .38-.91 (mean $r_{\mathrm{g}}=.63$ ), indicating genetic heterogeneity in the full item set. Meta-analyzing the two samples, we identify 255 genome-wide significant independent genomic regions, of which 138 are item-specific. Genetic analyses and genetic correlations with 33 external traits support genetic differences between the items. Hierarchical clustering analysis identifies two genetically homogeneous item clusters denoted depressed affect and worry. We conclude that the items used to measure neuroticism are genetically heterogeneous, and that biological understanding can be gained by studying them in genetically more homogeneous clusters. 


\subsection{Introduction}

GWAS on psychological traits like major depressive disorder $\left(h^{2}{ }_{S N P}=0.06\right)^{139}$, intelligence $\left(h^{2}{ }_{S N P}\right.$ $=0.20)^{140}$ and neuroticism $\left(h^{2}{ }_{S N P} \text { range: } 0.09-0.15\right)^{131,141}$ have uncovered numerous associated variants. These genome-wide significant single-nucleotide polymorphisms (SNPs), however, explain only a small portion of the twin heritability $\left(h^{2}{ }_{t w i n}=0.40,0.54 \text {, and } 0.47 \text {, respectively }\right)^{4}$. The small individual SNP effects support the infinitesimal model, which assumes the involvement of many genetic variants (i.e., polygenicity), each of (very) small effect, such that large sample sizes are required to robustly detect them.

Genetic studies on psychological traits often adopt phenotypic composite scores, such as a sumscore or binary case-control status, which summarize information contained in multiple items or symptoms. In psychological research, composite scores have proven useful e.g. in directing therapeutic intervention, and in predicting future school/job performance. However, the items or symptoms collectively operationalizing one trait can be very diverse in nature. For instance, in personality inventories for neuroticism, items vary from 'feeling miserable' and 'experiencing mood swings' to 'feeling guilty' and 'worry too long after an embarrassing experience'. Similarly, diagnostic symptoms for major depressive disorder (MDD) vary from 'increase in appetite', 'irritable mood', and 'fatigue' to 'insomnia', 'excessive guilt', and 'suicidal ideation'92. Previous studies $^{93,111,142}$ have shown that items or symptoms underlying the same composite score can indeed differ considerably with respect to e.g. their relations to external risk factors, their impact on impairment, and their underlying biology. In the context of gene-finding studies, power to detect associated variants is potentially lost when the summed items or symptoms are biologically heterogeneous. Specifically, the use of composite scores in gene-finding studies directs the focus of analysis to those variants that affect the majority of aggregated items, i.e., "global variants". The genetic signal of "local" variants, affecting only one or a few of the aggregated items, is severely diluted $^{85}$. That is, if summed items or symptoms are genetically heterogeneous, then GWAS analysis of their sum may yield a mix of diluted signals, which will bear resemblance to the infinitesimal model.

One can investigate the genetic homogeneity of items or symptoms underlying a sum-score or a case-control status by studying the genetics of the individual items. If items prove to be genetically heterogeneous, identification of more homogeneous subsets or clusters of items may be expedient to optimize the statistical power to disentangle genetic determinants. Indeed, detection of local variants, affecting e.g. only one cluster of items but not others, might facilitate biological understanding.

Here, we use data on 12 dichotomous neuroticism items (Supplementary Table 1 Eysenck Personality Questionnaire - Revised Short Form ${ }^{88}$ ), to investigate whether the items used to operationalize neuroticism are genetically homogeneous. Given its high correlation with depression $\left(r=0.58\right.$; genetic correlation $\left.r_{g}=0.60\right)$ and anxiety $\left(r=0.75 ; r_{g}=0.77\right)^{143,144}$, neuroticism is considered an important phenotype in psychiatric genetic research ${ }^{145}$. Indeed, neuroticism items often resemble items used to measure (anxious) depression (see Supplementary Table 1 for a 
substantive overview). For the present study, data was obtained from two samples of the UK Biobank cohort ${ }^{35}$ (sample 1: first UK Biobank release, available since 2015; sample 2: data added in the second UK Biobank release, available since July 2017; item-specific $N$ range sample 1: 106,218-109,017; sample 2: 260,083-266,896; see Methods; Supplementary Tables 1-2). We use sample 2 to replicate basic findings of sample 1, and then meta-analyze the results of both samples to compare item-specific genetic signals to the signal obtained in genetic analysis of the sum score. Our study demonstrates that item-level analyses supplement genetic sum-score analysis, as the 12 neuroticism items often show only moderate genetic overlap. Furthermore, we identified two genetically distinct item clusters, which may prove useful targets of investigation in future genetic analyses.

\subsection{Results}

\section{Phenotypic analyses}

Phenotypic analyses of sample 1 showed that the 12 neuroticism items correlated positively with each other (.17-.54, Supplementary Fig. 2.1), and with the weighted sum-score ('sum-score' henceforward; .51-.68, see Methods for information on how the sum-score was constructed). Associations of all 12 items and the sum-score with external (demographic/psychological) variables had largely the same sign, but occasionally showed considerable differences in magnitude (Supplementary Tables 3-4; Supplementary Figs. 2-3).

\section{Genetic correlations between items}

We performed 13 GWASs (12 items + sum-score) on sample 1 and applied bivariate LD score regression ${ }^{116,146}$ on the summary statistics to compute genetic correlations $\left(r_{g}\right)$ between all 13 phenotypes (upper triangle Fig. 2.1; Supplementary Data 1; see Methods for QC and technical details). Between items, $r_{\mathrm{g}}$ 's ranged from a low 0.38 (IRR/WORR-EMB; $\mathrm{SE}=0.048$ ) to a high $0.91\left(\mathrm{MOOD} / \mathrm{FED}-\mathrm{UP} ; \mathrm{SE}=0.030\right.$, mean $\left.r_{\mathrm{g}}=.64\right)$, and none of the $95 \%$ confidence intervals included 1 . These results indicate genetic heterogeneity in the full item set. To replicate these findings, we conducted the same 13 GWASs in sample 2 (lower triangle Fig. 2.1). The $r_{g}$ 's between the exact same items measured in both samples were all close to 1 (diagonal Fig. 2.1; all 95\% confidence intervals included 1) indicating that the genetic signal is highly similar across the two samples. In addition, the correlation between inter-item $r_{g}$ 's in samples 1 and 2 was 0.97, confirming a highly similar $r_{\mathrm{g}}$ pattern across the two samples. 


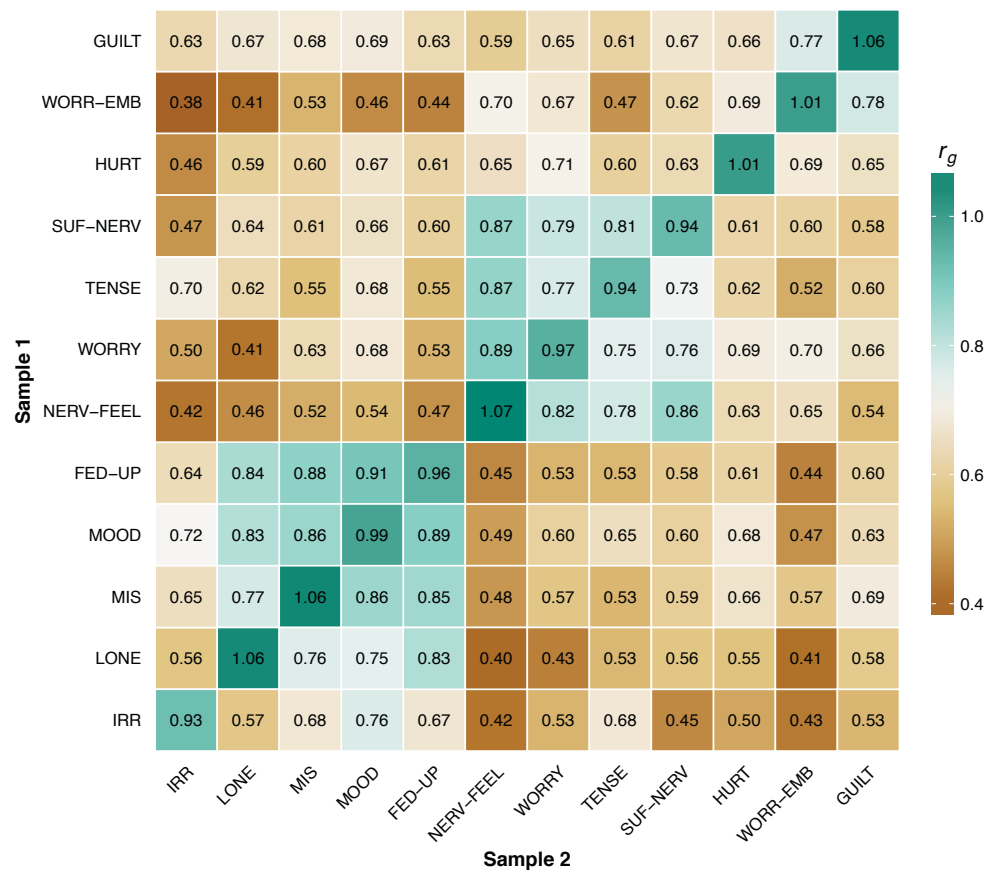

Figure 2.1. Genetic correlations between the 12 neuroticism items

Genetic correlations between the 12 neuroticism items within both samples (sample 1 is presented in the upper triangle and sample 2 in the bottom triangle). The genetic correlations of the same items measured in both samples are presented on the diagonal. All reported genetic correlations, computed with bivariate LD score regression, are significantly different from zero after Bonferroni correction $\left(P<2.22 \times 10^{-4}\right)$.

For the genetic correlations between the same items (diagonal) all $95 \%$ confidence intervals included 1, emphasizing concordance in genetic signal across samples. For all other, crossphenotype genetic correlations, none of the $95 \%$ confidence intervals included 1 , indicating that all genetic correlations deviated from unity (Supplementary Data 2). See Supplementary Table 1 for a description of the item labels.

\section{GWAS meta-analyses}

Having replicated the genetic heterogeneity in the set of neuroticism items, we moved on, for reasons of robustness, to conduct meta-analysis on the GWAS results of the two samples for all 12 items ( $N$ range: $366,301-375,913)$ and the sum-score $(N=380,060)$ to allow comparison between item-level GWAS results and results obtained in GWAS of the sum-score (Supplementary Table 5). 
In the 13 phenotypes, a total of 16,825 SNPs were genome-wide significant (GWS: $P<5 \times 10^{-}$ ${ }^{8}$ ), of which 2,474 were located in inversions on chromosomes 8 and 17, as reported previously for the neuroticism sum-score ${ }^{71,141,147}$. All GWS variants had the same direction of effect in both metaanalyzed samples. The 16,825 detected variants tagged 493 lead SNPs (see Methods for definition of lead SNPs), mapping to 255 independent genomic regions (based on clumping using an $r^{2}$ threshold of 0.1; Supplementary Data 2-17; Supplementary Figs. 4-17; see Methods; $\mathrm{http}: / /$ fuma.ctglab.nl/ $\left.{ }^{148}\right)$. Of the total 255 regions, 117 were GWS for the sum-score, and 6 to 44 regions (median $=32$ ) were GWS for individual items. Genetic signal varied considerably between items, with some (e.g., LONE, SUF-NERV) showing only a few GWS associations, while others (e.g., IRR, MIS, MOOD, NERV-FEEL, WORRY) showed $>35$ GWS genetic regions. Furthermore, of all 255 regions, 138 were GWS in item-level analyses only and not for the sum-score (Supplementary Data 2), and 42 were GWS in the sum-score analysis while no GWS association was observed for any of the items.

The 493 lead SNPs implicated 908 genes through positional mapping, eQTL mapping and/or chromatin interactions (see Methods; Supplementary Data 18-30). Of these 908 genes, 473 were only associated to one or more individual item(s) and not to the sum-score ${ }^{131,132,141,149}$ (Supplementary Fig. 18a).

Liability-scale SNP-based heritability estimates $\left(h^{2}{ }_{S N P}\right)$ for the 13 phenotypes, established using LD score regression (LDSC) $)^{146}$, ranged from $8 \%(\mathrm{SE}=0.0042)$ to $12 \%(\mathrm{SE}=0.0054)$ (Supplementary Fig. 19), and all estimates deviated significantly from 0.

In studying overlap in genetic signal between the 13 phenotypes (Methods; Supplementary Note 1), sign concordance tests showed that effect signs of top SNPs were largely concordant across the 13 phenotypes (Supplementary Fig. 20; Supplementary Data 31). However, Fisher's exact tests revealed that the exact top SNPs overlapped only moderately between the 13 phenotypes (Supplementary Fig. 21; Supplementary Data 32), indicating that the most strongly associated SNPs differed considerably between individual measures.

\section{Gene-based analysis}

By combining the genetic signal of all SNPs in a gene (while accounting for LD between SNPs), gene-based analyses can implicate genes that may go unnoticed in SNP-based analyses. Using the $P$ values from the GWA meta-analyses (see Methods), we conducted gene-based analyses in MAGMA $^{150}$ on all 13 phenotypes. In total, 654 genes reached GWS $\left(P<2.73 \times 10^{-6}\right)$, of which 388 overlapped with genes implicated in SNP-based analyses (Supplementary Data 33-46; Supplementary Figs. 18b, 22). 275 of the MAGMA genes were associated to the neuroticism sumscore, whereas 29 (LONE) to 172 (WORRY) genes (median =96) were GWS for individual items. Of the total of 654 genes, 379 were item-specific, i.e., only reached GWS in one or more individual items, indicating genetic heterogeneity between the 12 items, and between the items and their sum (Supplementary Data 33; Supplementary Fig. 18c). 
Overall, the results from the gene-based analyses suggest a role for both "global" genes (i.e., affecting (almost) all items and the composite score) and "local" genes, (i.e., affecting only one or a subset of items). In Table 2.1, a selection of such global and local genes is highlighted. Combined, SNP- and gene-based analyses on all 13 phenotypes implicated 1,247 genes, of which 651 were not identified in analysis of the sum-score (Supplementary Figs. 18d).

\section{Gene-set analysis}

While associations to individual SNPs or genes may differ between specific items and their summed score, it is possible that similar gene-sets and pathways are implicated. To test this, we used the results of the gene-based analyses as input for MAGMA's gene-set analysis ${ }^{150}$. For each of the 13 phenotypes we tested 7,244 gene-sets, comprising curated and gene ontology (GO) genesets derived from MsigDB ${ }^{151}$, and 53 tissue expression profiles ${ }^{152}$. The sum-score was significantly $\left(P<0.05 / 7,297=6.85 \times 10^{-6}\right)$ associated to $9 \mathrm{GO}$ gene-sets, whereas $8 \mathrm{GO}$ gene-sets were associated to one or more individual items, but not to the sum-score (Supplementary Data 47; Fig. 2). Noteworthy, 4 out of 9 GO gene-sets that were found to be associated to the sum-score were also associated to individual items, indicating that the sum-score and the individual items at least in part implicate the same biological processes.

The neuroticism sum-score was associated with genes expressed in 6 brain tissue types, all of which were also associated to at least one of the individual items. In addition, 2 brain tissue types, amygdala and caudate basal ganglia, were only identified in item-level gene-set analyses. Overall, while some gene-sets were implicated by multiple sources (Fig. 2), the gene-set analyses also confirmed the presence of genetic heterogeneity between the 12 neuroticism items and the sumscore. 


\begin{tabular}{|c|c|c|c|c|}
\hline Gene & $\mathrm{CHR}$ & Position & Implicated by & Notes \\
\hline SPPL2C & 17 & $43,922,256$ & All 13 phenotypes & $\begin{array}{l}\text { Only gene GWS associated to all } 13 \text { neuroticism phenotypes. Located in known } \\
\text { inversion on chromosome } 17 \text {. Associated to e.g. red blood cell count and Parkinson's } \\
\text { disease }{ }^{151,152} \text {. }\end{array}$ \\
\hline MAPT & 17 & $43,971,702$ & 11 of 13 phenotypes & $\begin{array}{l}\text { Promotes microtubule assembly and stability. Previously linked to intelligence }{ }^{63} \text { and } \\
\text { neurodegenerative disorders (e.g. Parkinson's and Alzheimer's disease }{ }^{153,154} \text { ). }\end{array}$ \\
\hline DRD2 & 11 & $113,280,317$ & 11 of 13 phenotypes & $\begin{array}{l}\text { Previously associated to neuroticism sum-score }{ }^{141} \text {. D2-receptors are thought to be } \\
\text { involved in reward processing. }\end{array}$ \\
\hline GRM8 & 7 & $126,078,652$ & 8 items + sum-score & $\begin{array}{l}\text { Not associated to neuroticism before. Has been associated to the response to } \\
\text { selective serotonin reuptake inhibitors (SSRI) in depressed individuals }{ }^{155} \text {. }\end{array}$ \\
\hline SORCS3 & 10 & $106,400,859$ & $\begin{array}{l}\text { Items in the depressed affect } \\
\text { cluster and sum-score, but not } \\
\text { items in the worry cluster }\end{array}$ & $\begin{array}{l}\text { Signal is driven primarily by } 3 \text { items (MIS, MOOD, FED-UP), all part of the depressed } \\
\text { affect cluster. SORCS3 has been associated to depression }{ }^{141} \text {, and is involved in } \\
\text { neuropeptide receptor activity. }\end{array}$ \\
\hline CADM2 & 3 & $85,008,133$ & $\begin{array}{l}\text { Items in the worry cluster and } \\
\text { sum-score, but not items in the } \\
\text { depressed affect cluster }\end{array}$ & $\begin{array}{l}\text { Most strongly associated gene for the worry cluster }\left(P=2.24 \times 10^{-23}\right) \text {, and GWS for all } \\
\text { items in this cluster. Linked to BMI in multiple studies }{ }^{156,157} \text { (see genetic correlations } \\
\text { between both clusters and BMI in Fig. 3). }\end{array}$ \\
\hline Region A & 3 & $\begin{array}{l}49.215-50.226 \\
\mathrm{Mb}\end{array}$ & Depressed affect items & $\begin{array}{l}\text { Genes in this region (e.g., C3orf84, RHOA, MST1, APEH) were previously linked to e.g. } \\
\text { Crohn's disease }{ }^{158} \text { and blood protein levels }{ }^{159} \text {. A subset of the genes in this region } \\
\text { (e.g. IP6K1, CAMKV, SEMA3F) have been associated with educational attainment }{ }^{160} \text {. }\end{array}$ \\
\hline Region $\mathrm{B}$ & 3 & $\begin{array}{l}50.264-53.080 \\
\mathrm{Mb}\end{array}$ & Worry items & $\begin{array}{l}\text { Genes in this region (e.g. ALAS1, STAB1, GNL3, ITIH4, TMEM110) have been linked to } \\
\text { schizophrenia and autism spectrum disorder in earlier studies }{ }^{161,162} \text {. }\end{array}$ \\
\hline
\end{tabular}


Note Table 2.1. All genes that were GWS in gene-based analyses in MAGMA ${ }^{150}$ on all 13 neuroticism phenotypes are reported in Supplementary Data 33.

$\mathrm{CHR}=$ Chromosome on which the gene is located; Position $=$ Start position of the gene in base pairs (for regions A \& B start and end positions are reported).

depressed affect and worry refer to two separate sets of 4 items each, i.e., LONE/MIS/MOOD/FEDUP, and NERV-FEEL/WORRY/TENSE/SUF-NERV, respectively. See Supplementary Table 1 for a description of the item labels.

\section{Functional annotation}

A comparison of functional consequences, chromatin state, and regulatory functions of all the SNPs in LD with one of the independent significant SNPs identified in item-level versus sum-score analysis is provided in Supplementary Tables 6-8 and Supplementary Fig. 23.

For the sum-score we identified 36 GWS exonic non-synonymous SNPs (ExNS), of which 29 overlapped with the 134 ExNS SNPs identified in item-level analyses (Supplementary Data 48). 10 of these overlapping ExNS SNPs are located in a well-known inversion on chromosome $17^{141}$. Overall, 105 ExNS SNPs were specifically associated with one or more of the individual items and went unnoticed in sum-score analysis, showing that item-level analyses superadded to the identification of SNPs that are highly likely to have functional consequences. As an example, we highlight two ExNS SNPs, not GWS for the neuroticism sum-score ${ }^{165}$ that may be viable candidates for functional follow-up. Of all ExNS SNPs associated to neuroticism items, rs45510500, located in exon 42 of KIAA1109, had the highest CADD score (35). rs45510500 is a missense mutation that leads to an amino acid change of Arginine to Tryptophan. The second SNP, rs3130618 in exon 3 of GPANK1 with a CADD score of 34, is a missense mutation resulting in an Arginine to Leucine change. rs 3130618 has a regulome database score of $1 \mathrm{f}$, implying that it is likely to affect binding and to affect expression of a gene target.

\section{Genetically homogeneous item clusters}

We performed a hierarchical clustering on the inter-item $r_{g}$ 's of sample 1 to see whether the items could be grouped into genetically homogeneous clusters (Fig. 2.1). This analysis revealed two clusters (1: LONE/MIS/MOOD/FED-UP 2: NERV-FEEL/WORRY/TENSE/SUF-NERV), with mean $r_{\mathrm{g}}=.84$ (range: $0.77-0.91$ ) within clusters, and a much lower mean $r_{\mathrm{g}}=.59$ (range: $0.38-$ 0.77 ) between items from different clusters. The identified clusters coincide with the dimensions depressed affect and worry previously identified in factor analysis of the full EPQ-R neuroticism scale $^{166}$. 


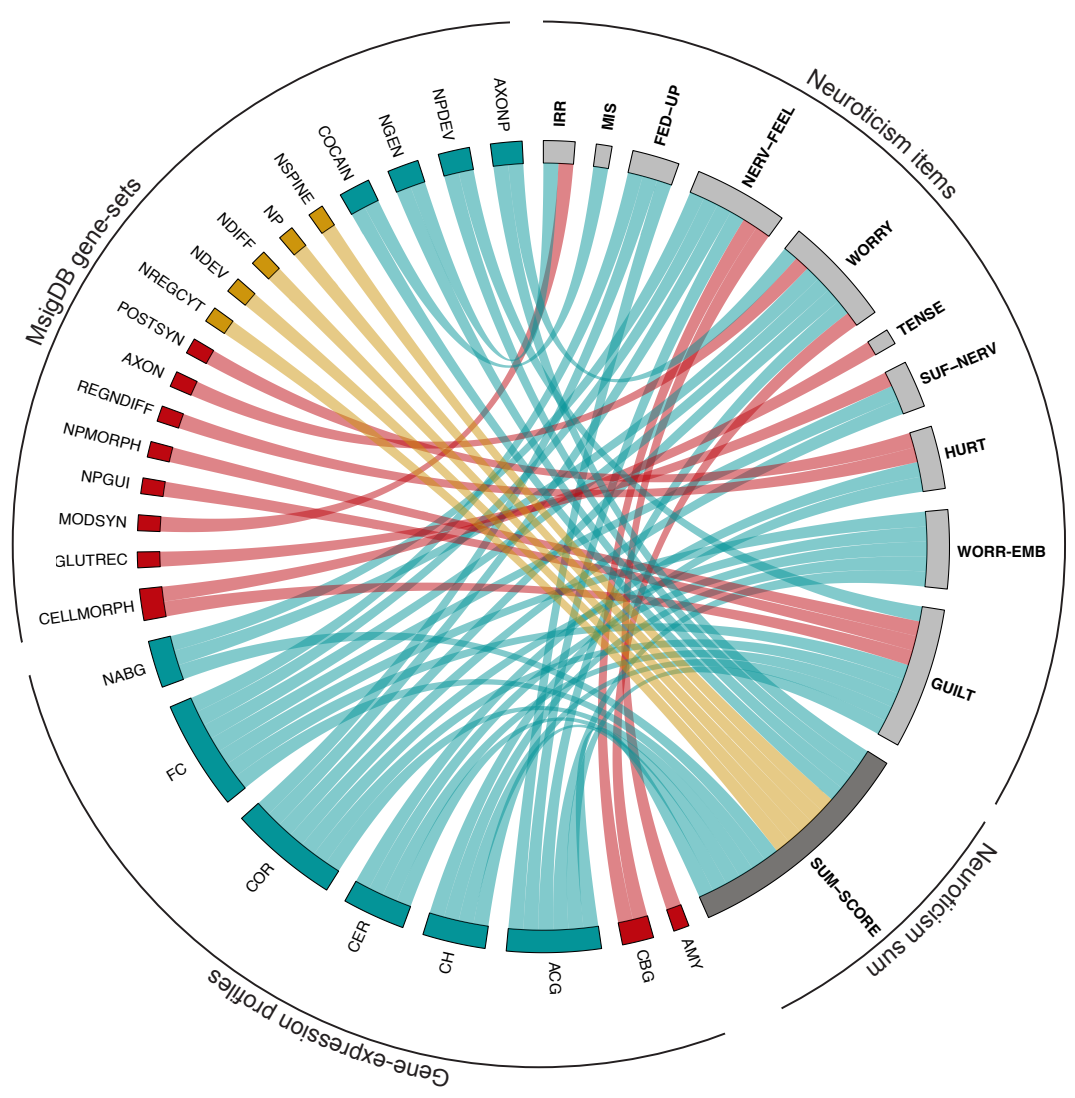

Figure 2.2. Circle plot of statistically associated gene-sets

Of the 7,297 gene-sets tested (MsigDB gene-sets and gene-expression profiles), this circle plot presents all gene-sets that were significantly associated (Bonferroni corrected $P$ value threshold: $0.05 / 7,297=6.85 \times 10^{-6}$ ) to at least one of the individual neuroticism items or to the sum-score. Each tract connects a gene-set to the phenotype with which it is associated. Tracts colored in red indicate gene-sets that were associated to individual items, but not to the sum-score. In contrast, tracts colored in yellow indicate gene-sets that were associated to the neuroticism sum-score, but not to any of the individual items. Blue tracts refer to gene-sets that were found to be associated to the sum-score and at least one of the individual items.

See Supplementary Data 47 for a description of the gene-sets included in this figure. See Supplementary Table 1 for a description of the item labels.

We then applied the item order that emerged from the cluster analysis on sample 1 to the genetic correlational results from sample 2, and observed the same two item clusters (lower triangle of Fig. 2.1), with very similar within and between item $r_{g}$ 's (overall mean $r_{g}=0.62$; range $r_{\mathrm{g}}$ 's: $0.40-0.89$; 
within clusters mean $r_{g}=0.80$, range: $0.73-0.89$; outside of clusters mean $r_{g}=0.58$, range: 0.40 0.78). The genetically homogenous clusters thus replicated robustly within the UKB sample. All items had a relatively high $r_{g}$ with the sum-score (range: $0.73-0.89$ ), but items assigned to clusters correlated even higher with their respective cluster, while $r_{\mathrm{g}}$ 's with the opposite cluster were considerably lower (difference $>.20$ ), confirming genetic heterogeneity within the full item set (Supplementary Fig. 24; Supplementary Data 1). Genetic analysis of such genetically homogenous clusters may reveal genetic signals that are important for multiple items in one cluster but not for items in the second cluster, as we recently showed in ${ }^{165}$.

\section{Genetic correlations with external traits}

Neuroticism is linked to numerous social, behavioral, and psychiatric traits ${ }^{131}$. We therefore calculated $r_{\mathrm{g}}$ 's of the 12 items, the sum-score, and the two identified item clusters with 33 external traits (Supplementary Data 49) using cross-trait LD score regression ${ }^{116}$. In line with previous findings for neuroticism sum-scores ${ }^{131,132,141}$, all items correlated negatively with subjective wellbeing (-.67 - -.45, sum-score: -.65), and positively with major depressive disorder (.35-.66, sumscore: .65), depressive symptoms (.42-.83, sum-score: .79), and anxiety disorders (.51-.78, sumscore: .76; Fig. 2.3; Supplementary Data 50). These strong positive $r_{\mathrm{g}}$ 's are presumably at least partly due to the substantial overlap in content between neuroticism items on the one hand and depression/anxiety items on the other (see Supplementary Table 1), which reduces the operational distinctness of these phenotypes.

In contrast, $r_{\mathrm{g}}$ 's of individual items to other external traits were not all in the same direction (e.g., IQ, BMI). Here, relying merely on sum-score information would be misleading: sum-score $r_{\mathrm{g}}$ 's can be small while item-specific $r_{\mathrm{g}}$ 's are substantial yet opposite $\left(r_{\mathrm{g}}>|.17|\right.$; see e.g., BMI, hip and waist circumference).

The magnitude of $r_{\mathrm{g}}$ 's associated with the items assigned to the clusters depressed affect and worry varied substantially for many traits (e.g., depressive symptoms, subjective well-being). Some of the traits showed genetic overlap with items of one of the clusters, but not with items from the other cluster (e.g., anorexia, schizophrenia, educational attainment, ADHD), whereas a few traits even showed GWS genetic correlations with items in both clusters yet in opposite directions (e.g., BMI, waist and hip circumference). Smoking related phenotypes (i.e., ever smoker, smoking cessation) showed significant correlations only to items of the depressed affect cluster, and not to items of the worry cluster. These results clearly indicate that biological insight may be gained by analyzing these genetically homogeneous clusters separately. 


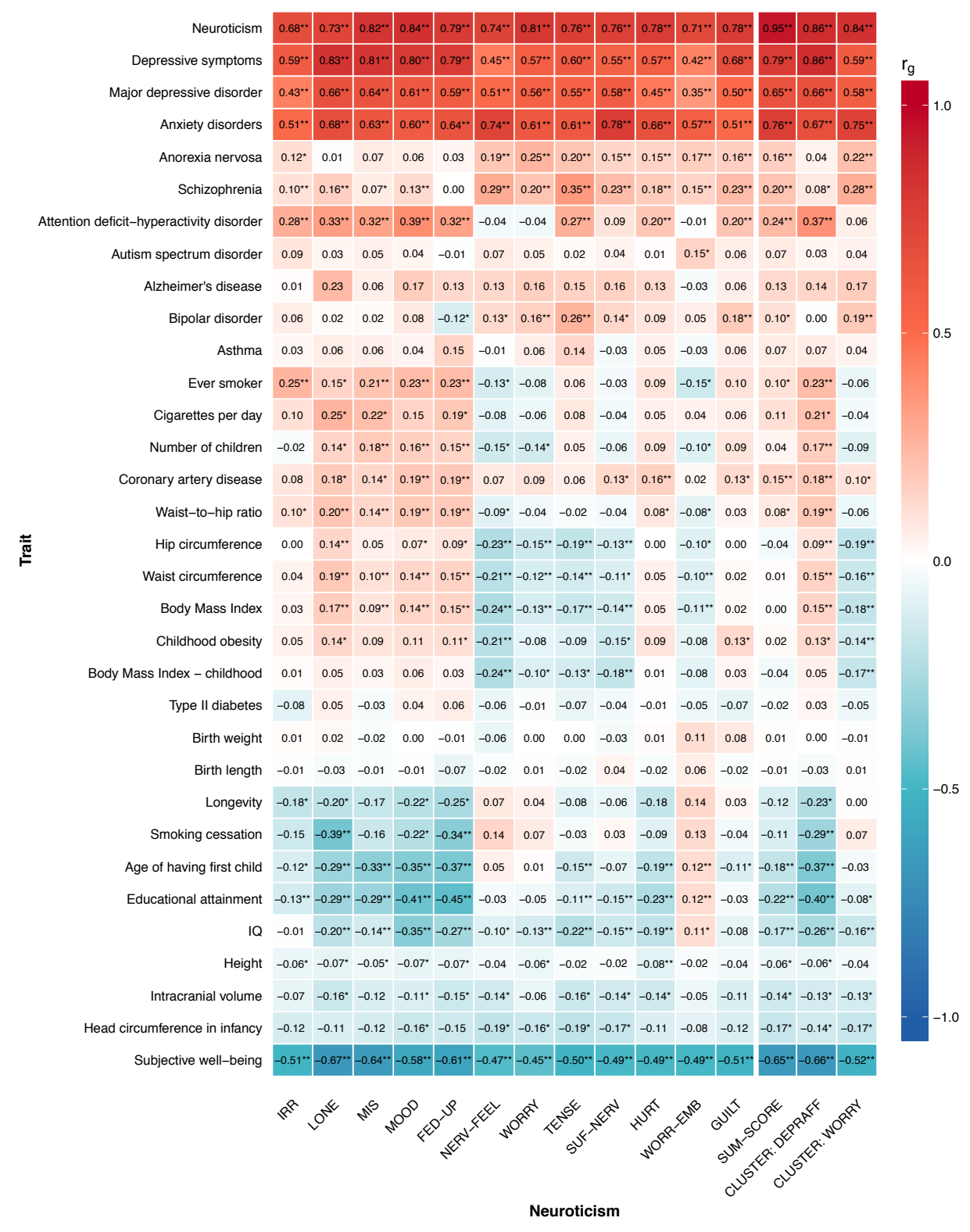

Figure 2.3. Genetic correlations of 12 neuroticism items, the sum-score and the two item clusters with 33 external traits 
Figure 2.3 (continued)

Genetic correlations of neuroticism items (see Supplementary Table 1 for a description of the item labels), sum-score, and the item clusters depressed affect (CLUSTER: DEPRAFF) and worry (CLUSTER: WORRY; $x$-axis) with 33 other traits ( $y$-axis; Supplementary Data 49) computed with cross-trait LD score regression. $P$ values and $95 \%$ confidence intervals for the $r_{g}$ 's are provided in Supplementary Data 50. Exact values of the genetic correlations are shown in the cells.

${ }^{*} P<0.01$; **Bonferroni corrected $P$ value threshold: $P<1.01 \times 10^{-4}(0.05 /(15 \times 33))$

\subsection{Discussion}

In summary, we have shown that items used to measure the personality trait neuroticism are genetically heterogeneous, with genetic overlap often being only moderate (i.e., $r_{\mathrm{g}}<.60$ ), and we replicated this in a second sample. We then proceeded to conduct GWAS meta-analyses on all 12 neuroticism items and their sum-score in $\geq 366,276$ individuals. We identified 138 item-specific loci implicating 1,247 genes, revealing considerable genetic variation between items. We identified two genetically distinct item clusters denoted depressed affect and worry. Within clusters, items were genetically strongly correlated, and items from different clusters showed distinct genetic correlational patterns to external traits.

The current findings motivate investigations into the genetic heterogeneity of items in other instruments used to gauge complex psychological traits. Neuroticism is only one of many psychological traits for which composite scores are calculated that are based on the aggregation of item or symptom scores. As a clinical example, consider the DSM-V diagnosis for major depressive disorder (MDD). This diagnosis is based on a list of 9 diverse symptoms (at least four of which are "aggregated" symptoms, reflecting problems on either end of the spectrum, e.g., "insomnia or hypersomnia", "increase or decrease in appetite", see Supplementary Note 2 for the full list of symptoms). To qualify for a depression diagnosis, at least 5 of these symptoms should be endorsed for at least two weeks, a procedure that can result in people with very different symptom profiles obtaining the same diagnosis ${ }^{111,112}$. In subsequently using the diagnostic status as dependent variable in GWAS, the assumption is that these symptoms are genetically similar. The phenotypic heterogeneity of the symptoms does, however, like with neuroticism, raise questions about their alleged genetic homogeneity. As yet, genetic heterogeneity between depression symptoms has only been addressed in the context of twin studies ${ }^{123}$.

Similarly, many psychological instruments measuring quantitative traits are known to be phenotypically multidimensional. For instance, the multidimensionality of cognitive ability is evident in the many subscales characterizing renowned IQ tests like the Wechsler Adult Intelligence Scale (WAIS ${ }^{167,168}$ ). Twin and family studies show that these multidimensional phenotypes are also genetically multidimensional, and that dimension-specific genetic effects can be substantial ${ }^{169-173}$. 
In sum, while all these psychological instruments have proven useful in therapeutic settings and in e.g. predicting school/job performance, the known phenotypic heterogeneity and multidimensionality begs the question whether an overall sum-score operationalization is expedient in gene finding studies, as there is generally no a priori reason to assume that the subscales, items, or symptoms are genetically similar. By studying the genetic architecture of individual subscales or items or symptoms, we can determine whether the apparent genetic complexity of psychiatric traits is (at least partly) introduced by our choice of operationalization. Item- or symptom-level analyses need not become the standard, but they can inform investigation and construction of genetically homogeneous subsets, as we have shown in the present study.

Our study highlights the purpose and relevance of item or symptom-level analyses as a means to inspect genetic homogeneity, and to identify subsets of genetically similar items or symptoms that can confidently be summed and used as input in future gene-finding enterprises.

\subsection{Methods}

\section{UK Biobank sample}

The UK Biobank Study is a major data resource, containing phenotypic measures from 503,325 participants and genetic data from 489,212 participants (July 2017 release) ${ }^{35}$. The data was released in two phases (May 2015 and July 2017), and based on this we created two separate samples on which we performed the GWA analyses.

Sample 1 consists of individuals for whom the data was released in May 2015 ( N=110,328; Supplementary Table 1), whereas sample 2 consists of all individuals that were added in the $2^{\text {nd }}$ release (July 2017; N=270,178; Supplementary Table 2). Written informed consent was obtained from all participants and the UK Biobank received ethical approval from the National Research Ethics Service Committee North West-Haydock (reference 11/NW/0382). The current study was conducted under UK Biobank application number 16406.

For the analyses in this study, we excluded all individuals of non-Caucasian ancestry (based on genetic principal components). Therefore, principal components from the 1000 Genomes reference populations ${ }^{174}$ were projected onto the called genotypes available in UK Biobank. Those participants whose projected principal component score was closest to the average score of the European 1000 Genomes (based on the Mahalanobis distance) were identified as European. We excluded European subjects with a Mahalanobis distance $>6$ SD. Additionally, subjects were filtered out based on relatedness, discordant sex, sex aneuploidy, and withdrawn consent. Finally, individuals were excluded from analyses if responses to more than 3 (of 12) of the neuroticism items were missing. The effective sample in our meta-analysis consisted of 380,506 individuals (205,556 females and 174,950 males; see Supplementary Table 5 for item-specific sample sizes). At the time of test completion, participants' age ranged between 40 and $73(\mathrm{M}=56.91 ; S D=7.93)$. 
For both samples specified above, we used newly imputed data from the $2^{\text {nd }}$ release, on approximately 96 million genetic variants. Imputation was performed by the UK Biobank, using a reference panel that included the UK10K haplotype panel as well as the Haplotype Reference Consortium reference panel. Based on recommendations by the UKB we excluded all variants imputed from the UK10K reference panel, as technical errors may have occurred during the imputation process. The imputed data was converted to hard-called genotypes using a certainty threshold of 0.9 .

\section{Neuroticism phenotype}

Neuroticism was measured using the Eysenck Personality Questionnaire, Revised Short Form (EPQ-R-S) ${ }^{88}$, consisting of 12 dichotomous items ("yes" or "no"). Participants completing less than 9 items were excluded from further analysis. We constructed a weighted neuroticism sum-score by adding up the individual item responses and dividing this sum by the total number of completed items (see Supplementary Tables 1-2 and 5 for the endorsement rates of the individual items in both samples, as well as in the full sample). Prior to analysis, the sum-score was standardized to have mean 0 and variance 1 .

\section{Cluster scores}

Scores for the depressed affect cluster were obtained by summing the scores on the four items "Do you often feel lonely?", "Do you ever feel 'just miserable' for no reason?", "Does your mood often go up and down?", and "Do you often feel 'fed-up'?". Scores on the worry cluster were obtained by summing the scores on the four items "Are you a worrier?", "Do you suffer from nerves?", "Would you call yourself a nervous person?", and "Would you call yourself tense or highly strung". Only participants with complete scores on all 4 items were included in GWA analyses of the cluster scores, resulting in sample sizes of $\mathrm{N}=361,768$ for depressed affect and $\mathrm{N}=350,356$ for worry.

\section{SNP-based analysis}

All genome-wide association (GWA) analyses were conducted separately on sample 1 and sample 2. GWA analyses of the 12 neuroticism items were conducted using logistic regression as implemented in PLINK 1.9 $9^{175,176}$, regressing the dichotomous neuroticism item responses on the imputed hard-called SNPs. Linear regression was used to analyze the neuroticism sum-score and both item cluster scores ${ }^{165}$. Sex, age, and townsend deprivation index (TDI; a measure based on postal code indicating material deprivation), were included in the analyses as covariates. Genotype array was only included as covariate in the analyses of sample 1 as the same array was used for all subjects in sample 2. Additionally, we included the first 10 genetic PCs as covariates to control for potential population stratification. Genetic PCs were computed separately for both samples using 
FlashPCA $2^{177}$ on individuals of European ancestry, after LD pruning and filtering out SNPs with $\mathrm{MAF}<0.01$ and genotype missingness $>0.05$.

Final data analysis was restricted to autosomal, bi-allelic SNPs with MAF $>0.0001$, high imputation quality (INFO score $\geq 0.9$ ) and low missingness ( $<0.05$ ), resulting in $10,847,151$ SNPs. Within each SNP-based GWA analysis, we applied the standard genome-wide significance threshold in European-descent samples ${ }^{178}$ of $P<5 \times 10^{-8}$. In addition, we indicate whether the genetic signal survived correction for the 13 phenotypes tested $\left(P<3.85 \times 10^{-9}\right.$; Supplementary

Data 2; Supplementary Fig. 4).

\section{Meta-analysis}

For all 13 phenotypes (12 items, sum), the SNP-based genome-wide association analyses were conducted separately on sample 1 and sample 2. To optimize the robustness of the genetic comparisons between the 13 phenotypes, we combined for all 13 phenotypes the GWA results from sample 1 and 2 using meta-analysis in METAL ${ }^{179}$. All SNP, gene, and gene-set analyses (Supplementary Data 2, 5-17, 18-30, 33-47) are thus based on the results from these metaanalyses.

\section{Excluded loci}

Based on visual inspection of the regional plots (LocusZoom) of all genomic loci identified in SNPbased meta-analyses of the 13 neuroticism phenotypes, we decided to further examine the plausibility of 6 of these loci, as they appeared to be driven by only one or two SNPs (Supplementary Data 3-4). First, we compared the allele frequencies of the lead SNPs in these loci that we observed in the UKB data to the allele frequencies reported by 1000 genomes and TOPMED, as a large difference might indicate genotyping errors (Supplementary Data 3). Secondly, we compared the $P$ values from GWA analyses in both samples to see whether the signal was driven by a subset of the data. Finally, differential LD across datasets might be another indicator of genotyping errors. Therefore, we selected all SNPs in LD $\left(r^{2}>0.6\right)$ with the lead SNPs in the 1000 Genomes data, and compared the LD in the 1000 Genomes data with LD (for the same combinations of SNPs) in the UKB data (Supplementary Data 4). None of these checks suggested genotyping errors of these SNPs. However, as the evidence for these loci was based on very few SNPs we decided to exclude them, and count the total number of unique genomic loci for all 12 items and the sum-score as $261-6=255$.

\section{Gene-based analysis}

Gene-based genome-wide association analyses (GWGAS) were conducted in MAGMA (http://ctg.cncr.nl/software/magma ${ }^{150}$ ), using the $P$ values from the GWA analyses as input. This gene-based analysis tests the joint signal of all SNPs in a gene with the phenotype, while accounting 
for LD between those SNPs, thus uncovering gene-level signal that may go unnoticed in SNP-based analyses. In total, 18,183 genes were covered by at least one SNP, and used in the GWGAS. Within each gene-based analysis, we applied a stringent Bonferroni correction for the number of tested genes, resulting in a genome-wide threshold for significance of $P<2.75 \times 10^{-6}(0.05 /$ number of genes tested). In addition, we indicate whether the gene-signal survives correction for the 13 phenotypes tested $\left(P<2.12 \times 10^{-7}\right.$; Supplementary Data 33; Supplementary Fig. 22).

\section{Gene-set analysis}

Using MAGMA ${ }^{150}$, we tested the association between all 13 neuroticism phenotypes and 7,297 gene-sets. Specifically, we tested for association with 7,244 gene ontology gene-sets (MsigDB version 6.0) and 53 tissue expression profiles (https://www.gtexportal.org/home/). For all gene-sets competitive $P$ values were computed, which result from the test whether the combined effect of genes in a gene-set is significantly larger than the combined effect of a same number of randomly selected genes (in contrast, self-contained $P$ values result from testing against the null hypothesis of no effect). We only report competitive $P$ values, which are more conservative compared to selfcontained $P$ values. Competitive $P$ values were Bonferroni corrected $\left(\alpha=0.05 / 7,297=6.85 \times 10^{-6}\right)$.

\section{SNP-based heritability and genetic correlations}

LD score regression (LDSC ${ }^{116,146}$ ) analyses were run on summary statistics obtained from GWA analyses to estimate the proportion of phenotypic variance attributable to all SNPs in the analyses (SNP-based heritability; $h^{2}{ }_{S N P}$ ). Bivariate LD Score regression (https://github.com/bulik/ldsc) on the GWAS summary statistics was used to estimate the genetic correlations between all neuroticism phenotypes. Significance of genetic correlations between all neuroticism phenotypes was determined by applying a Bonferroni corrected threshold of $P=2.22 \times 10^{-4}(0.05 /(15 \times 15)=0.05 / 225$; Fig. 1.1 and Supplementary Figure 24 combined).

The genetic correlations between the 15 neuroticism phenotypes and 33 sundry traits for which summary statistics were available, were also calculated using LD score regression. We corrected for multiple testing through a stringent Bonferroni correction leading to a $P$ value threshold of $P<$ $1.01 \times 10^{-4}(0.05 /(15 \times 33)=0.05 / 495)$.

\section{Genetic overlap tests for top associated SNPs}

Fisher's exact tests were used to examine the overlap in the top associated SNPs across all pairwise combinations of neuroticism items and the sum-score. Prior to this analysis, we applied LD-based pruning on the summary statistics from all items to ensure that SNPs were independent (a subset of 10,000 UKB participants was used as a reference set; $r^{2}=0.8$ ). Sign tests were used to establish whether signs of top associated SNPs were in the same direction across all neuroticism items and the sum-score. Here we used clumping $\left(r^{2}=0.1\right.$ with window size $\left.500 \mathrm{~kb}\right)$ based on the association 
$P$ values to ensure that the most strongly associated variants were not lost. As clumping results in different SNP sets for different items, sign test results are not symmetrical for pairs of items. For both tests, we used multiple $P$ value thresholds, including the conventional GWS threshold $\left(5 \times 10^{-}\right.$ $\left.{ }^{8}\right)$ and a conservative threshold correcting for the 13 phenotypes tested $\left(3.85 \times 10^{-9}\right)$.

We acknowledge that smoother measures than thresholding are available. For example, the Rank-Rank-Hypergeometric-Overlap (RRHO) test ${ }^{180}$ displays overlap in a more nuanced manner. However, as we generally worked with 13 phenotypes, resulting in $13 \times 13-13=156$ combinations (considering that some of the thresholded tests were not symmetrical), a method like RRHO would yield 156 individual figures from which it would be difficult to discern patterns in the results.

\section{Functional annotation and gene-mapping using FUMA}

The FUMA GWAS platform (http://fuma.ctglab.nl/148) uses GWAS summary statistics to functionally map, annotate, prioritize, visualize, and interpret GWAS results. We used the summary statistics from GWA meta-analyses on the 12 individual items and the neuroticism sum-score as input for FUMA.

FUMA first defined independent significant SNPs which have a genome-wide significant $P$ value $\left(5 \times 10^{-8}\right)$ and are independent at $r^{2}<0.6$. Subsequently, lead SNPs were defined by retaining those independent significant SNPs that were independent from each other at $r^{2}<0.1$. Next, risk loci were defined by merging physically overlapping lead SNPs or lead SNPs whose LD blocks were closer than $250 \mathrm{~kb}$ apart. A consequence of this definition of risk loci is that the same locus may be discovered for different phenotypes included in the study, while the lead SNPs are different.

All SNPs in LD (>0.6) with one of the independent significant SNPs were used in annotation. Functional consequences were obtained by performing ANNOVAR gene-based annotation using Ensembl genes. In addition, potential regulatory functions are indicated by the RegulomeDB score $^{181}$ (with lower scores indicating a higher probability of having a regulatory function) and by 15-core chromatin states predicted by ChromHMM ${ }^{182}$ for 127 tissue/cell types ${ }^{183}$.

All SNPs in genomic risk loci that were GWS, or in LD $(>0.6)$ with one of the independent GWS SNPs were mapped to genes in FUMA ${ }^{148}$ using either of three strategies.

The first strategy we applied, positional mapping, was used to map SNPs to genes based on the physical distances (i.e., within $10 \mathrm{~kb}$ window) from known protein coding genes in the human reference assembly (GRCh37/hg19).

The second strategy, eQTL mapping, is used to link SNPs to genes with which these SNPs show a significant eQTL association (i.e., allelic variation at the SNP affects the expression of that gene). This strategy is based on information from 3 data repositories (GTEx, Blood eQTL browser, and BIOS QTL browser), and uses cis-eQTLs, which can map SNPs to genes that lie up to $1 \mathrm{Mb}$ apart. We applied a false discovery rate (FDR) of 0.05 to define significant eQTL associations.

Finally, using chromatin interaction, SNPs were mapped to genes based on a significant chromatin interaction between a genomic region in a risk locus and promoter regions of genes 
(250bp up and 500bp downstream of transcription start site (TSS)). Unlike eQTL mapping, chromatin interaction mapping has no distance boundary and can involve long-range interactions. Currently, Hi-C data of 14 tissue types are included in FUMA ${ }^{184}$. Generally, chromatin interactions are defined in a certain resolution (e.g., $40 \mathrm{~kb}$ ) such that interacting regions may span multiple genes. All SNPs within these regions would be mapped by this method to genes in the corresponding interaction region. To further prioritize candidate genes from chromatin interaction mapping, we integrated predicted enhancers and promoters in 111 tissue/cell types from the Roadmap Epigenomics Project ${ }^{183}$; chromatin interactions are selected in which one region involved in the interaction overlaps with predicted enhancers and the other region overlaps with predicted promoters in $250 \mathrm{bp}$ up- and $500 \mathrm{bp}$ downstream of the TSS site of a gene. A FDR of $1 \times 10^{-5}$ was applied to define significant interactions.

\section{Data availability}

Our policy is to make genome-wide summary statistics (sumstats) publicly available. Sumstats from the GWA analyses of all neuroticism phenotypes are available for download at https://ctg.cncr.nl/. 


\subsection{Supplementary information}

\section{Additional information on genetic overlap tests for top associated SNPs}

Before conducting the sign concordance tests to establish whether signs of top associated SNPs were in the same direction across the 13 phenotypes, we used clumping $\left(r^{2}=0.1\right.$ with window size $500 \mathrm{~kb}$ ) based on the association $P$ values to ensure that the most strongly associated variants were not lost. As clumping results in different SNP sets for different phenotypes, sign tests results are not symmetrical for pairs of phenotypes. To explicate the followed procedure:

1. Select two items I1 \& I2

2. Retain only SNPs that survived clumping for item I1

3. Filter out all variants with $>$ threshold for phenotype I1

4. Of the selected SNPs, compute the number of SNPs that have the same sign (i.e., concordant) for both I1 and I2

5. The proportion we report is: prop $=\frac{N_{\text {concordant }}}{N_{\text {total }}}$ (both numbers in this equation only contain SNPs below the specified P threshold for item I1, mentioned in step 3)

6. Repeat for all combinations of items and sum-score

In addition, we repeat the procedure with the difference that clumping and $P$ value filtering is based on item I2 instead of item I1. This results in heatmaps (visualizing the proportion of concordant sign SNPs) that are not symmetric, which can be seen from the number of SNPs reported in each cell.

Prior to performing Fisher's exact tests to examine the overlap in the top associated SNPs across the 13 Neuroticism phenotypes, we applied LD-based pruning on the summary statistics from all items to ensure that SNPs were independent (a subset of 10,000 individuals from the UK biobank, the UK10K set, was used as a reference set; $r^{2}=0.8$ ). Using the SNPs that survived pruning, we then created (for each combination of items, and for each $P$ value threshold) a table like the one depicted below. The categories in the table below are exclusive, meaning that a SNP in cell $\mathbf{A}$ is not included in the count of cells $\mathbf{B}$ and $\mathbf{C}$.

\begin{tabular}{|l|l|}
\hline $\begin{array}{l}\text { A } \\
\text { Number of SNPs }<\text { Pthreshold for both } \\
\text { items }\end{array}$ & $\begin{array}{l}\text { B } \\
\text { Number of SNPs }<\text { Pthreshold for item 2 \& } \\
>\text { Pthreshold for item 1 }\end{array}$ \\
\hline $\begin{array}{l}\text { C } \\
\text { Number of SNPS }<\text { Pthreshold for item 1 \& } \\
>\text { Pthreshold for item 2 }\end{array}$ & $\begin{array}{l}\text { Number of SNPS }>\text { Pthreshold for both } \\
\text { items }\end{array}$ \\
\hline
\end{tabular}




\section{Diagnostic criteria Major Depressive Disorder}

Below we list the symptoms used in the diagnosis of Major Depressive Disorder, as described more extensively in the Diagnostic and Statistical Manual (DSM-5 ${ }^{92}$ ).

1) Depressed mood

2) Diminished interest or pleasure

3) Weight loss or weight gain, or decrease or increase in appetite

4) Insomnia or hypersomnia

5) Psychomotor agitation or retardation

6) Fatigue or loss of energy

7) Feelings of worthlessness or excessive or inappropriate

8) Diminished ability to think or concentrate, or indecisiveness

9) Recurrent thoughts of death, recurrent suicidal ideation

\section{Acknowledgements and notes}

\section{Acknowledgments}

This work was funded by The Netherlands Organization for Scientific Research (NWO MagW VIDI 452-12-014; NWO VICI 453-14-005). The analyses were carried out on the Genetic Cluster Computer, which is financed by the Netherlands Organization for Scientific Research (NWO: 48005-003), by the VU University (Amsterdam, The Netherlands), and by the Dutch Brain Foundation, and is hosted by the Dutch National Computing and Networking Services SurfSARA. This research has been conducted using the UK Biobank Resource, application number 16406. We thank the participants and researchers who collected and contributed to the data. Summary statistics will be made available for download from http://ctglab.vu.nl

\section{Author Contributions}

M.N. performed the analyses. S.vd.S, D.P., and M.N. conceived the study. S.S. QC-ed the UKB data and developed the analysis pipeline. K.W. constructed the FUMA tool for biological annotation. M.N., S.vd.S, and D.P. wrote the paper. All authors discussed the results and commented on the paper. 



\section{3 \\ Meta-analysis of genome- wide association studies for neuroticism in 449,484 individuals identifies novel genetic loci and pathways}

Mats Nagel $^{1,2 \dagger}$, Philip R Jansen ${ }^{1,3 \dagger}$, Sven Stringer ${ }^{1}$, Kyoko Watanabe ${ }^{1}$, Christiaan A de Leeuw ${ }^{1}$, Julien Bryois ${ }^{4}$, Jeanne E Savage ${ }^{1}$, Anke R Hammerschlag${ }^{1}$, Nathan G Skene ${ }^{5}$, Ana B MuñozManchado $^{5}$, the 23andMe Research Team ${ }^{6}$, Tonya White ${ }^{3}$, Henning Tiemeier ${ }^{3,8}$, Sten Linnarsson ${ }^{5}$, Jens Hjerling-Leffler ${ }^{5,6}$, Tinca JC Polderman ${ }^{1}$, Patrick F Sullivan ${ }^{4,9,10}$, Sophie van der Sluis ${ }^{1,2 \S}$, Danielle Posthuma ${ }^{1,2 \S^{*}}$

1. Department of Complex Trait Genetics, Center for Neurogenomics and Cognitive Research, Amsterdam Neuroscience, Vrije Universiteit Amsterdam, The Netherlands

2. Department of Clinical Genetics, Section Complex Trait Genetics, Amsterdam Neuroscience, VU University Medical Center, Amsterdam, the Netherlands

3. Department of Child and Adolescent Psychiatry, Erasmus University Medical Center, Rotterdam, the Netherlands

4. Department of Medical Epidemiology and Biostatistics, Karolinska Institutet, Stockholm, Sweden

5. Laboratory of Molecular Neurobiology, Department of Medical Biochemistry and Biophysics, Karolinska Institutet, Stockholm, Sweden

6. UCL Institute of Neurology, Queen Square, London WC1N 3BG, UK

7. 23andMe, Inc., Mountain View, CA, USA

8. Department of Social and Behavioral Science, Harvard TH Chan School of Public Health, Boston, MA, USA

9. Department of Genetics, University of North Carolina, Chapel Hill, USA

10. Department of Psychiatry, University of North Carolina, Chapel Hill, USA

$\dagger$ These authors contributed equally to this work

$\S \quad$ These authors jointly supervised this work

Published in: Nature Genetics (2018). Volume 50, doi: 10.1038/s41588-018-0151-7 


\section{Abstract}

Neuroticism is an important risk factor for psychiatric traits, including depression ${ }^{185}$, anxiety ${ }^{144,186}$, and schizophrenia ${ }^{187-189}$. At the time of analysis, previous genome-wide association studies $^{129,131,141,149,190,191}$ (GWAS) reported 16 genomic loci associated to neuroticism ${ }^{131,141,191}$. Here, we conducted a large GWAS meta-analysis $(\mathrm{N}=449,484)$ of neuroticism and identified 136 independent genome-wide significant loci (124 novel at the time of analysis), implicating 599 genes. Functional follow-up analyses showed enrichment in several brain regions and involvement of specific cell types, including dopaminergic neuroblasts $\left(P=3.49 \times 10^{-8}\right)$, medium spiny neurons $\left(P=4.23 \times 10^{-8}\right)$ and serotonergic neurons $\left(P=1.37 \times 10^{-7}\right)$. Gene-set analyses implicated three specific pathways: neurogenesis $\left(P=4.43 \times 10^{-9}\right)$, behavioral response to cocaine processes $\left(P=1.84 \times 10^{-7}\right)$, and axon part $\left(P=5.26 \times 10^{-8}\right)$. We show that neuroticism's genetic signal partly originates in two genetically distinguishable subclusters ${ }^{192}$ (depressed affect and worry, the former being genetically strongly related to depression, $r_{g}=0.84$ ), suggesting distinct causal mechanisms for subtypes of individuals. Mendelian randomization analysis showed uni- and bidirectional effects between neuroticism and multiple psychiatric traits. These results enhance neurobiological understanding of neuroticism and provide specific leads for functional follow-up experiments. 


\subsection{Results}

The meta-analysis of neuroticism comprised data from the UK Biobank Study (UKB, full release ${ }^{193} ; n=372,903$; Methods and Supplementary Fig. 1-2), 23andMe, Inc. ${ }^{194}(n=59,206)$, and the Genetics of Personality Consortium (GPC1 ${ }^{129} ; n=17,375$; Methods, $n=449,484$ in total). In all of the samples, neuroticism was measured through (digital) questionnaires (Methods and Supplementary Note). To achieve optimal power, SNP associations were subjected to metaanalysis using METAL $^{179}$, with weighting by sample size (Methods). We chose to perform metaanalysis on the available samples rather than use a two-stage discovery-replication strategy, because Skol et al. ${ }^{195}$ showed that this is almost always more powerful, even though less correction for multiple testing is required in the replication stage.

The quantile-quantile (Q-Q) plot of the genome-wide meta-analysis on 449,484 subjects and $14,978,477$ SNPs showed inflation (linkage disequilibrium score regression (LDSC) ${ }^{146}: \lambda_{\mathrm{GC}}=1.65$, mean $\chi^{2}$ statistic=1.91; Fig. 3.1a and Supplementary Table 1), yet the LDSC intercept (1.02; standard error $(\mathrm{SE})=0.01)$ and ratio $(2.1 \%)$ both indicated that the inflation was largely due to true polygenicity and the large sample size ${ }^{196}$. The $\lambda_{\mathrm{GC}}$ value of 1.65 is consistent with values observed in recent large-sample GWAS $(n>100,000)$ for diverse and polygenic traits (Supplementary Note). The LDSC SNP-based heritability $\left(h^{2}{ }_{S N P}\right)$ of neuroticism was $0.100(\mathrm{SE}=0.003)$. The GWAS meta-analysis identified 9,745 genome-wide significant (GWS) SNPs $\left(P<5 \times 10^{8}\right)$, of which 157 and 2,414 were located in known associated inversions on chromosomes 8 and 17131,141,191, respectively (Fig. 3.1b and Supplementary Fig. 3; see Supplementary Table 2 for cohort-specific information).

We used FUMA ${ }^{148}$, a tool to functionally map and annotate results from GWAS (Methods), and extracted 170 independent lead SNPs (158 new; see the Methods for definition of lead SNPs) that mapped to 136 independent genomic loci (124 new at the time of analysis) (Methods, Supplementary Tables 3-8, and Supplementary Note). Of all the lead SNPs, 4 were in exonic regions, 88 were in intronic regions, and 52 were in intergenic regions. Of the 17,794 SNPs in high linkage disequilibrium (LD) with one of the independent significant SNPs (see the Methods for definition), most were intronic $(9,147 ; 51.4 \%)$ or intergenic $(5,460 ; 30.7 \%)$, and $3.8 \%$ were annotated as potentially having a functional impact, with $0.9 \%$ (155 SNPs) being exonic (Fig. 3.1c and Supplementary Table 9; see Supplementary Tables 10 and 11 for an overview of the chromatin state and regulatory functions of these SNPs). Of these 155 SNPs, 70 were exonic nonsynonymous (ExNS) (Table 3.1 and Supplementary Table 12). 


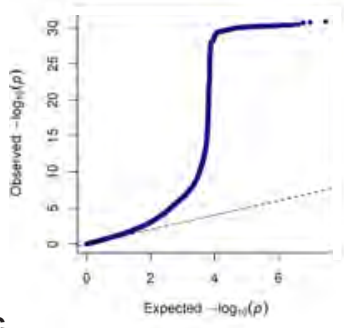

C

Functional annotation SNPs
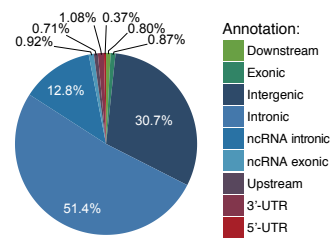

d

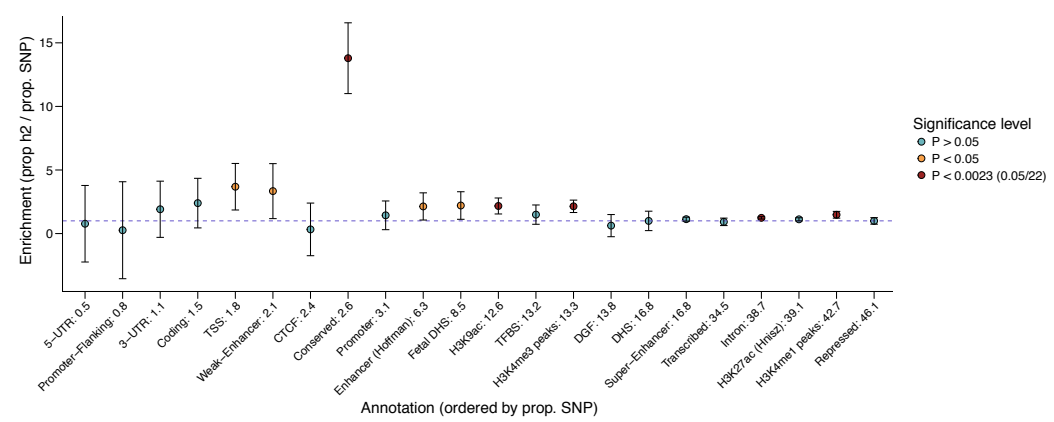

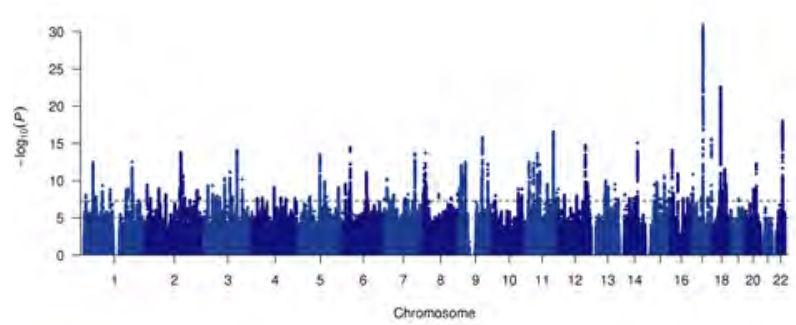

Chromatin state
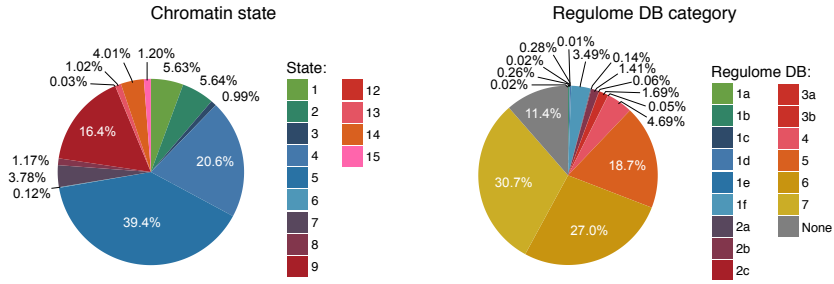

Figure 3.1. SNP-based associations with neuroticism in the GWAS meta-analysis.

(a) Quantile-quantile plot of the SNP-based associations with neuroticism ( $\mathrm{N}=449,484$ individuals). SNP $P$ values were computed in METAL using a two-sided, weighted z-score method. (b) Manhattan plot showing the - $\log _{10}$ transformed $P$ value of each SNP on the y-axis and base pair positions along the chromosomes on the $\mathrm{x}$-axis $(\mathrm{N}=449,484$ individuals). SNP $P$ values were computed in METAL using a two-sided, weighted $z$-score method. The dashed line indicates genome-wide significance $\left(P<5 \times 10^{-8}\right)$, the dotted line the threshold for suggestive associations $\left(P<1 \times 10^{-5}\right)$. (c) Pie charts showing the distribution of functional consequences of SNPs in linkage disequilibrium (LD) with genome-wide significant lead SNPs in the meta-analysis, the minimum chromatin state across 127 tissue and cell types and the distribution of regulome DB score, a categorical score between 1a and 7, indicating biological evidence of a SNP being a regulatory element, with a low score denoting a higher likelihood of being regulatory. (d) Heritability enrichment of 22 functional SNP annotations calculated with stratified LD Score regression (summary statistics of the meta-analysis of neuroticism were used as input for this analysis). The circles signify the estimated enrichment, whereas the dashed line indicates enrichment of 1 . Error bars represent $95 \%$ confidence intervals. TSS, transcription start site; CTCF, CCCTC binding factor; DGF, digital genomic footprint; TFBS, transcription factor binding site; DHS, DNase I hypersensitivity site. 
The ExNS SNP with the highest combined annotation-dependent depletion ${ }^{197}$ (CADD) score (which indicates the likelihood of the SNP being deleterious; Methods) was rs17651549 (CADD score of 34), located on chromosome 17 in exon 6 of $M A P T$, with a GWAS $P$ value of $1.11 \times 10^{-28}$, in high LD with the lead SNP in that region $\left(r^{2}=0.97\right)$. rs17651549 is a missense mutation (c.1108 C > T:p.Arg370Trp) that leads to an arginine-to-tryptophan change with allele frequencies matching the inversion in that region. The ancestral $\mathrm{C}$ allele is associated with a lower neuroticism score (see Table 3.1 and Supplementary Table 12 for a detailed overview of all functional variants in genomic risk loci).

Stratified LDSC ${ }^{198}$ (Methods) showed significant enrichment for $h^{2}$ of SNPs located in conserved regions (enrichment $\left.=13.79, P=5.14 \times 10^{-16}\right), \quad$ intronic regions (enrichment $\left.=1.24, P=1.27 \times 10^{-6}\right)$, and trimethylated Lys4 on histone $\mathrm{H} 3$ (H3K4me3; enrichment $=2.14, P=1.02 \times 10^{-5}$ ) and acetylated Lys9 on histone H3 (H3K9ac; enrichment $\left.=2.17, P=3.06 \times 10^{-4}\right)$ regions $($ Fig. 3.1d and Supplementary Table 13).

\section{Polygenic score analysis}

Polygenic scores (PGSs) calculated using PRSice ${ }^{199}$ (clumping followed by $P$ value thresholding) and LDpred ${ }^{200}$ in three randomly drawn hold-out samples (UKB only, $n=3,000$ each; Methods) explained up to $4.2 \%\left(P=1.39 \times 10^{-30}\right)$ of the variance in neuroticism (Supplementary Fig. 4, Supplementary Table 14, and Supplementary Note). Although the current sample size is considered to be large for GWAS and PGSs can be calculated with relatively low standard errors, the variance explained by all SNPs combined in the PGSs was still relatively small, although this was not unexpected given the h2SNPhSNP2 of $10 \%$. Our current results thus have little predictive power in independent samples, mostly owing to the low average effect sizes of contributing SNPs, and indicate that the genetic architecture of neuroticism is extremely polygenic. We do note that our current meta-analysis did not include possible genetic interactions (as even with the current sample sizes power would be limited) but that adding these in the future may increase the predictive value of PGSs for neuroticism. 
Table 1. Exonic nonsynonymous variants in the genomic loci associated with neuroticism and in $L D\left(r^{2}>0.6\right)$ with one of the independent genome-wide significant SNPs

\begin{tabular}{|c|c|c|c|c|c|c|c|c|c|c|c|c|}
\hline rsID & Exon & Gene & A1 & MAF & gwas $P$ & $z$-score & $r^{2}$ & $\begin{array}{l}\text { Indep. Sign. } \\
\text { SNP }\end{array}$ & Locus & CADD & RDB & $\begin{array}{l}\text { Min. Chrom. } \\
\text { state }\end{array}$ \\
\hline rs41266050 & 14 & RABGAP1L & $T$ & 0.25 & $5.65 E-06$ & -4.54 & 0.84 & rs7536102 & 6 & 2.85 & 7 & 5 \\
\hline rs34605051 & 10 & KDM3A & $\mathrm{T}$ & 0.16 & 1.64E-07 & -5.24 & 0.99 & rs11127043 & 14 & 13.85 & NA & 4 \\
\hline rs2073498 & 3 & RASSF1 & $A$ & 0.11 & $2.71 \mathrm{E}-08$ & 5.56 & 0.98 & rs6776145 & 25 & 19.43 & 7 & 3 \\
\hline rs4434138 & 62 & STAB1 & A & 0.46 & $3.10 \mathrm{E}-07$ & 5.12 & 0.93 & rs2015971 & 26 & 5.48 & 5 & 4 \\
\hline rs66782572 & 1 & NT5DC2 & A & 0.46 & $2.65 \mathrm{E}-06$ & 4.70 & 0.67 & rs2015971 & 26 & 0.00 & 4 & 1 \\
\hline rs11177 & 3 & GNL3 & A & 0.38 & $2.49 \mathrm{E}-07$ & -5.16 & 0.75 & rs2015971 & 26 & 22.90 & NA & 1 \\
\hline rs6617 & 1 & SPCS1 & C & 0.41 & $1.96 \mathrm{E}-06$ & 4.76 & 0.65 & rs2015971 & 26 & 0.00 & $1 f$ & 1 \\
\hline rs1029871 & 5 & NEK4 & C & 0.38 & $2.29 \mathrm{E}-07$ & -5.17 & 0.75 & rs2015971 & 26 & 24.10 & $1 f$ & 2 \\
\hline rs678 & 12 & ITIH1 & A & 0.36 & $1.44 \mathrm{E}-06$ & 4.82 & 0.65 & rs2015971 & 26 & 25.90 & $1 f$ & 2 \\
\hline rs1042779 & 12 & $|T| H 1$ & A & 0.37 & 6.09E-06 & 4.52 & 0.63 & rs2015971 & 26 & 0.15 & $1 f$ & 2 \\
\hline rs198844 & 1 & HIST1H1T & C & 0.47 & $3.07 \mathrm{E}-08$ & -5.54 & 0.98 & rs198825 & 45 & 0.10 & $1 f$ & 5 \\
\hline rs200484 & 1 & HIST1H2BL & A & 0.13 & $1.53 \mathrm{E}-07$ & 5.25 & 0.61 & rs200965 & 46 & 2.68 & $1 f$ & 1 \\
\hline rs240780 & 39 & ASCC3 & C & 0.43 & 3.01E-08 & 5.54 & 0.96 & rs240769 & 49 & 19.95 & 7 & 4 \\
\hline rs11765552 & 11 & LMTK2 & A & 0.46 & $7.68 \mathrm{E}-08$ & 5.38 & 0.98 & rs34320230 & 55 & 12.24 & 6 & 4 \\
\hline rs10821128 & 3 & FAM120AOS & $\mathrm{T}$ & 0.33 & $2.73 \mathrm{E}-09$ & 5.95 & 0.99 & rs10821129 & 71 & 0.05 & 4 & 4 \\
\hline rs41274386 & 2 & FAM120AOS & T & 0.08 & 1.10E-07 & 5.31 & 0.66 & rs78046549 & 71 & 2.36 & 4 & 1 \\
\hline rs1055710 & 1 & FAM120AOS & A & 0.33 & $1.11 \mathrm{E}-09$ & -6.09 & 0.99 & rs10821129 & 71 & 0.05 & NA & 1 \\
\hline rs3816614 & 33 & LRP4 & T & 0.23 & $5.69 \mathrm{E}-07$ & 5.00 & 0.90 & rs7940441 & 84 & 22.70 & NA & 4 \\
\hline rs2030166 & 5 & NDUFS3 & T & 0.35 & $2.02 \mathrm{E}-10$ & -6.36 & 0.93 & rs11039389 & 84 & 3.13 & 6 & 4 \\
\hline rs1064608 & 13 & MTCH2 & C & 0.35 & $1.15 \mathrm{E}-10$ & -6.45 & 0.93 & rs11039389 & 84 & 25.40 & 6 & 4 \\
\hline rs12286721 & 13 & $A G B L 2$ & A & 0.45 & $7.81 \mathrm{E}-08$ & -5.37 & 0.78 & rs7107356 & 84 & 14.22 & $1 f$ & 5 \\
\hline rs3816605 & 5 & NUP160 & T & 0.46 & $5.68 \mathrm{E}-08$ & -5.43 & 0.75 & rs7107356 & 84 & 6.61 & 6 & 4 \\
\hline rs4926 & 7 & SERPING1 & A & 0.27 & $6.12 \mathrm{E}-07$ & 4.99 & 0.86 & rs73480560 & 85 & 23.50 & 5 & 4 \\
\hline rs11604671 & 6 & ANKK1 & A & 0.49 & $2.57 \mathrm{E}-10$ & -6.32 & 0.64 & rs2186800 & 88 & 1.39 & 5 & 4 \\
\hline rs2734849 & 8 & ANKK1 & A & 0.49 & $8.43 \mathrm{E}-10$ & 6.14 & 0.64 & rs2186800 & 88 & 0.00 & $3 a$ & 4 \\
\hline
\end{tabular}


Table 1. Exonic nonsynonymous variants in the genomic loci associated with neuroticism and in $L D\left(r^{2}>0.6\right)$ with one of the independent genome-wide significant SNPs

\begin{tabular}{|c|c|c|c|c|c|c|c|c|c|c|c|c|}
\hline rsID & Exon & Gene & A1 & MAF & gwas $P$ & z-score & $r^{2}$ & $\begin{array}{l}\text { Indep. Sign. } \\
\text { SNP }\end{array}$ & Locus & CADD & $\mathrm{RDB}$ & $\begin{array}{l}\text { Min. Chrom. } \\
\text { state }\end{array}$ \\
\hline rs1800497 & 8 & ANKK1 & $A$ & 0.20 & $8.45 E-06$ & 4.45 & 0.69 & rs11214607 & 88 & 0.81 & NA & 4 \\
\hline rs3825393 & 30 & MYO1H & $\mathrm{T}$ & 0.36 & $2.95 \mathrm{E}-07$ & -5.13 & 0.79 & rs2111216 & 94 & 10.93 & NA & 4 \\
\hline rs7298565 & 12 & UBE3B & $A$ & 0.48 & $2.24 \mathrm{E}-10$ & 6.34 & 0.76 & rs2111216 & 94 & 22.70 & 6 & 4 \\
\hline rs9593 & 9 & $M M A B$ & $A$ & 0.48 & $8.54 \mathrm{E}-10$ & -6.14 & 0.76 & rs2111216 & 94 & 0.53 & If & 4 \\
\hline rs8007859 & 7 & EXD2 & T & 0.39 & $2.28 \mathrm{E}-08$ & 5.59 & 0.80 & rs1275411 & 108 & 3.95 & 5 & 4 \\
\hline rs7156590 & 3 & RPS6KL1 & $\mathrm{T}$ & 0.37 & $2.79 E-07$ & 5.14 & 0.86 & rs3213716 & 110 & 19.46 & 5 & 4 \\
\hline rs35755513 & - & CSNK1G1 & $\mathrm{T}$ & 0.07 & $2.87 \mathrm{E}-08$ & 5.55 & 1.00 & rs35755513 & 114 & 23.90 & 4 & 1 \\
\hline rs12443627 & 1 & ENSG00000268863 & C & 0.37 & $1.28 \mathrm{E}-10$ & 6.43 & 0.77 & rs3751855 & 119 & 3.58 & $2 b$ & 1 \\
\hline rs9938550 & 7 & HSD3B7 & $A$ & 0.37 & $1.48 \mathrm{E}-10$ & -6.41 & 0.79 & rs3751855 & 119 & 0.04 & $1 d$ & 3 \\
\hline rs35713203 & 1 & ZNF646 & C & 0.38 & $3.67 \mathrm{E}-11$ & -6.62 & 0.98 & rs3751855 & 119 & 0.05 & $2 b$ & 3 \\
\hline rs7196726 & 1 & ZNF646 & $A$ & 0.38 & 1.29E-11 & -6.77 & 1.00 & rs3751855 & 119 & 0.00 & $2 b$ & 3 \\
\hline rs7199949 & 8 & PRSS53 & C & 0.38 & $1.32 \mathrm{E}-11$ & -6.77 & 1.00 & rs3751855 & 119 & 0.00 & $2 b$ & 2 \\
\hline rs3748400 & 12 & $\mathrm{ZCCHC14}$ & $\mathrm{T}$ & 0.23 & 8.83E-09 & -5.75 & 0.98 & rs2042395 & 122 & 24.00 & 5 & 4 \\
\hline rs12949256 & 1 & ARHGAP27 & $\mathrm{T}$ & 0.19 & 1.47E-23 & 10.00 & 0.73 & rs77804065 & 126 & 11.97 & 4 & 1 \\
\hline rs16940674 & 6 & CRHR1 & T & 0.23 & $5.24 \mathrm{E}-29$ & 11.18 & 0.97 & rs77804065 & 126 & 12.86 & $1 f$ & 5 \\
\hline rs16940681 & 13 & CRHR1 & C & 0.23 & $2.18 \mathrm{E}-30$ & 11.46 & 0.97 & rs77804065 & 126 & 1.76 & 4 & 5 \\
\hline rs62621252 & 1 & SPPL2C & T & 0.23 & $9.05 E-31$ & -11.53 & 0.97 & rs77804065 & 126 & 0.00 & 5 & 5 \\
\hline rs242944 & 1 & SPPL2C & $A$ & 0.44 & $2.88 \mathrm{E}-12$ & -6.98 & 1.00 & rs242947 & 126 & 0.00 & NA & 5 \\
\hline rs62054815 & 1 & SPPL2C & $A$ & 0.23 & $1.74 \mathrm{E}-30$ & 11.48 & 0.97 & rs77804065 & 126 & 0.00 & 5 & 5 \\
\hline rs12185233 & 1 & SPPL2C & C & 0.23 & $6.76 E-29$ & 11.16 & 0.96 & rs77804065 & 126 & 25.60 & $1 f$ & 5 \\
\hline rs12185268 & 1 & SPPL2C & A & 0.23 & $4.08 E-30$ & -11.40 & 0.97 & rs77804065 & 126 & 0.00 & $1 f$ & 5 \\
\hline rs12373123 & 1 & SPPL2C & T & 0.23 & $7.80 E-29$ & -11.14 & 0.97 & rs77804065 & 126 & 22.70 & $1 f$ & 5 \\
\hline rs12373139 & 1 & SPPL2C & $A$ & 0.23 & 2.19E-30 & 11.46 & 0.97 & rs77804065 & 126 & 0.53 & $1 f$ & 5 \\
\hline rs12373142 & 1 & SPPL2C & C & 0.22 & $2.60 \mathrm{E}-28$ & -11.04 & 0.97 & rs77804065 & 126 & 0.12 & If & 5 \\
\hline
\end{tabular}


Table 1. Exonic nonsynonymous variants in the genomic loci associated with neuroticism and in LD $\left(r^{2}>0.6\right)$ with one of the independent genome-wide

significant SNPs

\begin{tabular}{|c|c|c|c|c|c|c|c|c|c|c|c|c|}
\hline rsID & Exon & Gene & A1 & MAF & gwas $P$ & $z$-score & $r^{2}$ & $\begin{array}{l}\text { Indep. Sign. } \\
\text { SNP }\end{array}$ & Locus & CADD & RDB & $\begin{array}{l}\text { Min. Chrom. } \\
\text { state }\end{array}$ \\
\hline rs754512 & 1 & MAPT & A & 0.23 & $1.17 \mathrm{E}-28$ & -11.11 & 0.97 & rs77804065 & 126 & 2.39 & $1 d$ & 4 \\
\hline rs63750417 & 6 & MAPT & T & 0.23 & 4.89E-30 & 11.39 & 0.97 & rs77804065 & 126 & 8.68 & 5 & 4 \\
\hline rs62063786 & 6 & MAPT & A & 0.23 & $1.05 E-29$ & 11.32 & 0.97 & rs77804065 & 126 & 7.65 & 5 & 4 \\
\hline rs62063787 & 6 & MAPT & $\mathrm{T}$ & 0.23 & $4.57 E-30$ & -11.39 & 0.97 & rs77804065 & 126 & 0.00 & 5 & 4 \\
\hline rs17651549 & 6 & MAPT & $\mathrm{T}$ & 0.23 & $1.11 \mathrm{E}-28$ & 11.11 & 0.97 & rs77804065 & 126 & 34.00 & 1f & 4 \\
\hline rs10445337 & 8 & MAPT & T & 0.23 & $1.41 \mathrm{E}-28$ & -11.09 & 0.96 & rs77804065 & 126 & 9.93 & $1 f$ & 4 \\
\hline rs34579536 & 15 & KANSL1 & A & 0.23 & $1.92 \mathrm{E}-30$ & -11.47 & 0.96 & rs77804065 & 126 & 8.02 & $3 a$ & 3 \\
\hline rs34043286 & 8 & KANSL1 & A & 0.23 & $3.14 \mathrm{E}-30$ & -11.43 & 0.97 & rs77804065 & 126 & 15.71 & 4 & 4 \\
\hline rs4969391 & 14 & BAIAP2 & A & 0.16 & 1.69E-14 & 7.67 & 0.90 & rs56084168 & 128 & 12.58 & 4 & 4 \\
\hline rs2282632 & 11 & ASXL3 & A & 0.50 & $3.37 \mathrm{E}-08$ & -5.52 & 0.73 & rs10460051 & 129 & 1.54 & NA & 4 \\
\hline rs7232237 & 12 & $A S X L 3$ & A & 0.50 & 1.59E-08 & -5.65 & 0.84 & rs10460051 & 129 & 0.00 & 5 & 4 \\
\hline rs17522826 & 1 & TCF4 & A & 0.18 & $2.17 \mathrm{E}-10$ & 6.35 & 0.60 & rs10503002 & 133 & 14.22 & NA & 1 \\
\hline rs20551 & 15 & EP300 & A & 0.29 & $3.44 \mathrm{E}-18$ & -8.70 & 0.98 & rs9611519 & 138 & 3.23 & 5 & 4 \\
\hline rs739134 & 2 & C22orf46 & T & 0.19 & 4.55E-07 & 5.04 & 0.61 & rs761366 & 138 & 22.60 & $1 f$ & 4 \\
\hline
\end{tabular}

Note Table 1. SNP $P$ values and $z$ scores were computed in METAL using a weighted $z$-score method (two-sided test). Per-SNP $n$ values are reported in Supplementary Table 2 (for genome-wide significant SNPs) and in the publicly available summary statistics. rsID, rs number of the ExNS SNP; Exon, exon in which the SNP is located; Gene, nearest gene; A1, effect allele; MAF, minor allele frequency; GWAS $P$, SNP $P$ value in the GWAS meta-analysis; $\mathrm{z}$ score, $\mathrm{z}$ score from the GWAS meta-analysis; $r^{2}$, maximum $r^{2}$ of the SNP with one of the independent significant SNPs; Locus, index of the genomic risk locus; CADD, CADD score; RDB, RegulomeDB score; Min. Chrom. state, minimum chromatin state of the SNP. Results are reported on hg19 coordinates (GRCh37); NA, not available in RegulomeDB (alleles do not match). Genes containing multiple ExNS SNPs are annotated in bold. 


\section{Gene-mapping}

We used four strategies to link our SNP results to genes: positional, expression quantitative trait locus (eQTL), and chromatin interaction mapping (Methods) and genome-wide gene-based association study (GWGAS, using MAGMA ${ }^{150}$ ). GWGAS evaluates the joint association effect of all SNPs within a gene to yield a gene-based $P$ value. Based on our meta-analysis results, 283 genes were implicated through positional mapping, 369 were implicated through eQTL mapping, and 119 were implicated through chromatin interaction mapping (Fig. 3.2a and Supplementary Table 15). GWGAS identified 336 genome-wide significant genes $\left(P<2.75 \times 10^{-6}\right.$; Fig. 3.2b-c, Supplementary Table 16, and Supplementary Note), of which 203 overlapped with genes implicated by FUMA, resulting in 599 unique neuroticism-related genes. Of these, 50 were implicated by all four methods, of which 49 had chromatin interaction and eQTL associations in the same tissue or cell type (Fig. 3.2a and Supplementary Table 15).

Nineteen of the 119 genes implicated through chromatin interaction mapping are especially notable, as they were implicated via interactions between two independent genome-wide significant genomic risk loci. There were several chromatin interactions in seven tissue types (aorta, hippocampus, left ventricle, right ventricle, liver, spleen, and pancreas) across two risk loci on chromosome 6 (Fig. 3.3a). Two genes are present in locus 45 and were mapped by chromatin interactions from risk locus 46 ( $H F E$ and $H I S T 1 H 4 C$ ), and 16 genes encode histones in locus 46 and were mapped by interactions from locus 45 (Supplementary Table 15). One gene, XKR6, located on chromosome 8 in risk locus 61 is implicated by chromatin interactions in five tissue types (aorta, left ventricle, liver, pancreas, and spleen), including cross-locus interactions from locus 60 (Fig. 3.3b and Supplementary Table 15). This gene was also mapped by eQTLs in blood and transformed fibroblasts. Of the 19 genes mapped by two loci, 4 were located outside of the risk loci (HIST1H2AI, HIST1H3H, HIST1H2AK, and HIST1H4L) and 7 were also implicated by eQTLs in several tissue types (HFE in subcutaneous adipose, aorta, esophagus muscularis, lung, tibial nerve, sun-exposed skin, and thyroid; HIST1H4J in blood and adrenal gland; HIST1H4K, HIST1H2AK, HIST1H2BO, and XKR6 in blood). 

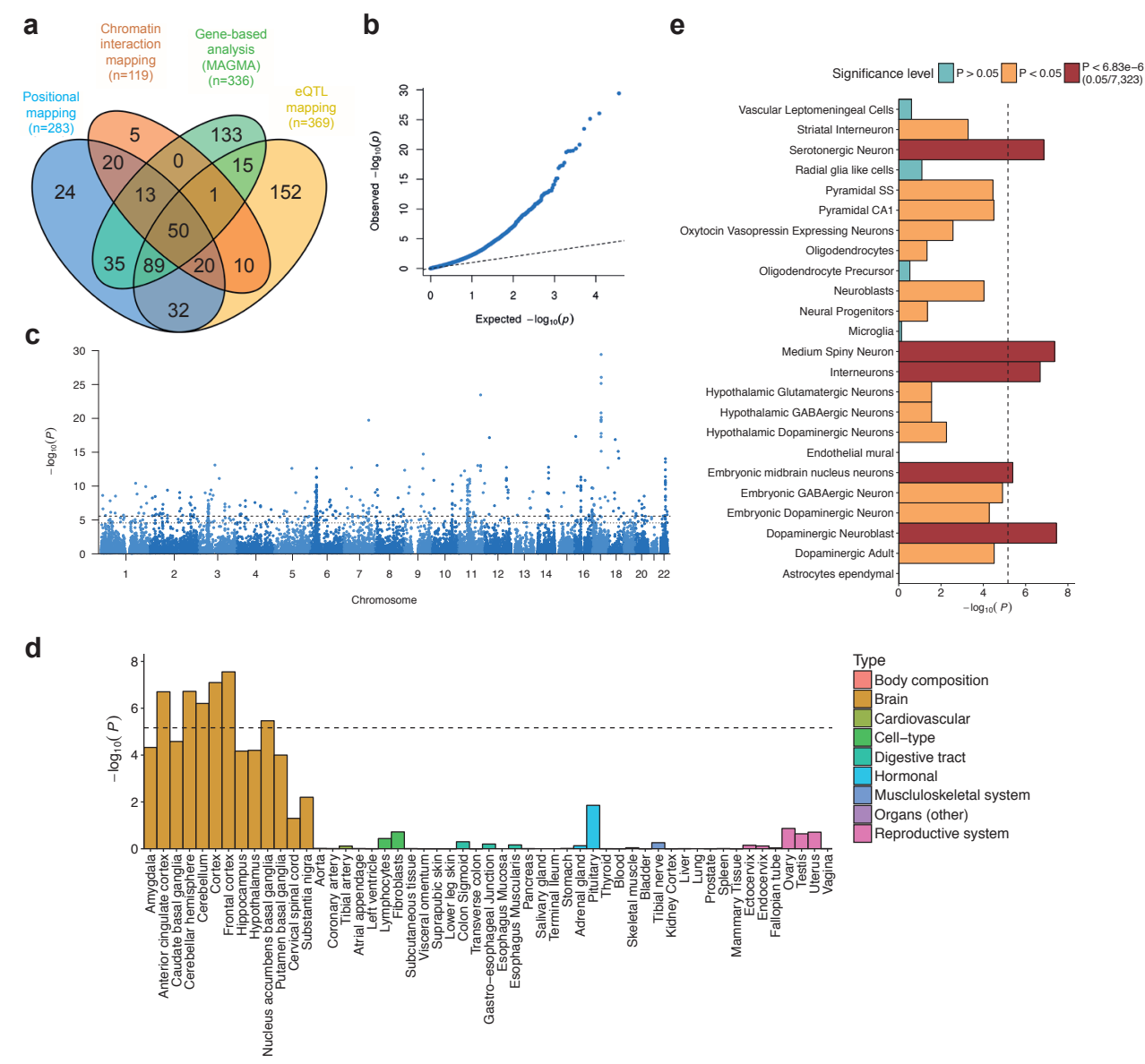

Figure 3.2. Mapping of genes and tissue- and cell expression profiles.

(a) Venn diagram showing overlap of genes implicated by positional mapping, eQTL mapping, chromatin interaction mapping, and genome-wide gene-based association (GWGAS). (b) Quantile-quantile plot of the GWGAS ( $\mathrm{N}=449,484$ individuals). Gene $P$ values were computed using MAGMA's gene-based test. (c) Manhattan plot of the GWGAS on neuroticism ( $\mathrm{N}=449,484$ individuals). Gene $P$ values were computed using MAGMA's gene-based test. The y-axis shows the - $\log 10$ transformed $P$ value of each gene, and the chromosomal position (start position) on the $\mathrm{x}$-axis. The dashed line indicates the threshold for genome-wide significance of the gene-based test $\left(P<2.75 \times 10^{-6}\right.$; $0.05 / 18,128)$, and the dotted line indicates the suggestive threshold $\left(P<2.75 \times 10^{-5}\right.$; $0.5 / 18,128$ ). (d) Gene expression profiles of identified genes for 53 tissue types. Expression data were extracted from the Genotype-Tissue Expression (GTEx) database. Expression values (RPKM) were $\log _{2}$ transformed with pseudocount 1 after winsorization at 50 and averaged per tissue. Gene-set tests for tissue expressions were calculated using MAGMA (Methods). (e) Enrichment of genetic signal for neuroticism in 24 cell types derived from mouse brain. The dashed line indicates the Bonferroni-corrected significance threshold $\left(P=0.05 / 7,323=6.83 \times 10^{-6}\right)$. 


\section{Gene-set analyses}

We used the gene-based $P$ values for gene set analysis in MAGMA ${ }^{150}$ and tested 7,246 predefined

gene sets derived from MSigDB ${ }^{151}$, gene expression profiles in 53 tissue types obtained from the Genotype-Tissue Expression (GTEx) Project ${ }^{201}$, and 24 cell-type-specific expression profiles using RNA-seq information ${ }^{202}$ (Methods). Neuroticism was significantly associated with genes predominantly expressed in six brain tissue types (Fig. 3.2d and Supplementary Tables 17 and 18) and with seven Gene Ontology (GO) gene sets, with the strongest association for neurogenesis $\left(P=4.43 \times 10^{-9}\right)$ and neuron differentiation $\left(P=3.12 \times 10^{-8}\right)$ (Supplementary Table 17). Conditional gene set analyses (Methods) suggested that three of the seven gene sets (neurogenesis, $P=4.43 \times 10^{-9}$; $\quad$ behavioral $\quad$ response $\quad$ to cocaine, $P=1.84 \times 10^{-7}$; axon part, $P=5.26 \times 10^{-8}$ ) had largely independent associations, implying a role in neuroticism (Supplementary Table 19). Conditional analyses of the tissue-specific expression ascertained general involvement of (frontal) cortex-expressed genes (Supplementary Fig. 5 and Supplementary Table 20).

Cell-type-specific gene set analysis showed significant association with genes expressed in multiple mouse-derived brain cell types (Fig. 3.2e, Methods, and Supplementary Table 21), with dopaminergic neuroblasts $\left(P=3.49 \times 10^{-8}\right)$, medium spiny neurons $\left(P=4.23 \times 10^{-8}\right)$, and serotonergic neurons $\left(P=1.37 \times 10^{-7}\right)$ showing the strongest associations. Conditional analysis indicated that these three cell types were also independently associated with neuroticism. 
a

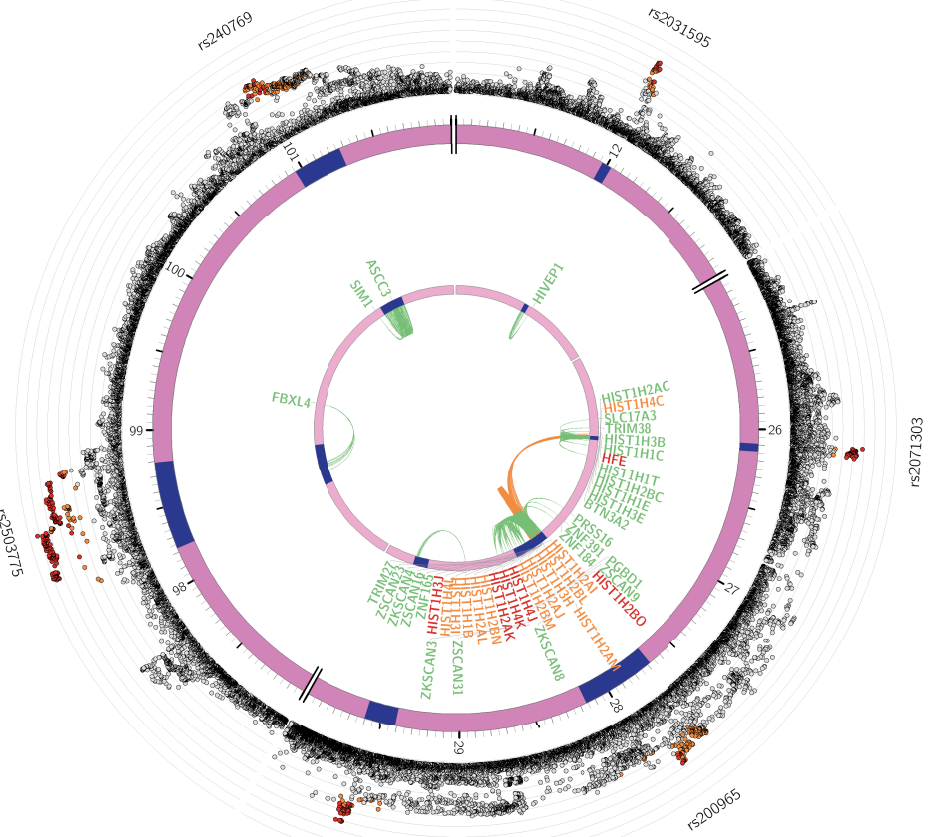

b

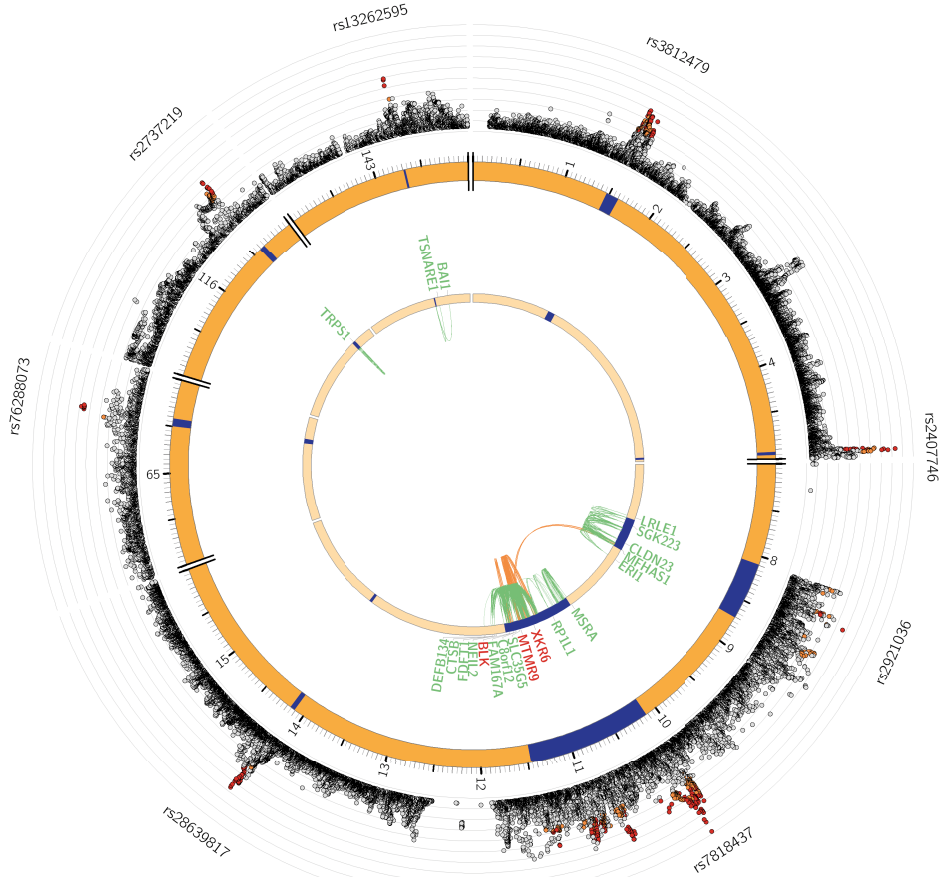


Figure 3.3. Genomic risk loci, eQTL associations and chromatin interaction for chromosome 6 and 8 , containing cross-locus interactions.

Circos plot showing genes on (a) chromosome 6 and (b) chromosome 8 that were implicated by the genomic risk (blue areas) loci by chromatin interaction (CTI; orange), eQTL (green) or implicated by both eQTL and CTI mapping (red). The outer layer shows a Manhattan plot containing the - $\log 10$ transformed $P$ value of each SNP in the GWAS meta-analysis of neuroticism $(\mathrm{N}=449,484$ individuals). Empty regions in the Manhattan plot layer indicate regions where no SNPs with $P<0.05$ are situated.

\section{Comparison to depression and neuroticism subclusters}

With the aim to further specify neuroticism's neurobiological interpretation, we compared the genetic signal of the full neuroticism trait to that of two genetically distinguishable neuroticism subclusters, 'depressed affect' and 'worry' (Methods), which we had previously established through hierarchical clustering of the genetic correlations between the 12 neuroticism items ${ }^{192}$. As a validation of the depressed affect dimension, we also compared the genetic signal of neuroticism and the two subclusters to that of depression. Genome-wide association analyses of the subclusters were conducted on the UKB data only (dictated by item-level data availability (Methods); depressed affect, $n=357,957$; worry, $n=348,219)$. For depression, our meta-analysis comprised data from the $\operatorname{UKB}^{203}\left(n=362,696\right.$; Supplementary Fig. 6), 23 andMe ${ }^{194}(n=307,354)$, and the Psychiatric Genetics Consortium ( $\mathrm{PGC}^{37} ; n=18,759$ ) (total $n=688,809$, which is the largest $n$ for depression thus far; $r_{\mathrm{g}}$ between samples: $0.61-0.80$; Methods and Supplementary Table 22; see the Supplementary Note for details on the depression GWAS results). Genetic correlations of neuroticism with all three phenotypes were considerable (depression, $r_{\mathrm{g}}=0.79$; depressed affect, $r_{\mathrm{g}}=0.88$; worry, $r_{\mathrm{g}}=0.87$; Supplementary Table 23). The positive genetic correlations between neuroticism and depression might in part be due to overlap in item content between the instruments used to gauge these phenotypes, reducing their operational distinctness ${ }^{192}$.

The subclusters showed notable differences in genetic signal (for example, exclusive genomewide significant associations on chromosomes 2 and 19 for depressed affect and on chromosomes 3 and 22 for worry; Supplementary Figs. 7-13 and Supplementary Tables 24-26). Of the 136 genetic loci associated with neuroticism, 32 were also genome-wide significant for depressed affect (7 shared with depression) but not for worry, and 26 were also genome-wide significant for worry (3 shared with depression) but not for depressed affect (Supplementary Fig. 13 and Supplementary Table 27). These results were mirrored by gene-based analyses (Supplementary Fig. 14, Supplementary Tables 28-30, and Supplementary Note), suggesting that part of neuroticism's genetic signal originated specifically in one of the two subclusters, possibly implicating different causal genetic mechanisms. To further verify the biological distinctness of the two clusters, cluster-specific functional annotation was conducted, which demonstrated that, with respect to SNPs that were highly likely to have functional consequences (ExNS), the clusters were 
(i) distinct and (ii) adding information to the results of neuroticism sum-score analysis (Supplementary Fig. 15, Supplementary Tables 31-34, and Supplementary Note).

To test whether the signal of the gene sets implicated in neuroticism originated from one of the specific subclusters, we conducted conditional analyses, correcting neuroticism for depressed affect and worry scores separately (Supplementary Fig. 16 and Supplementary Table 35). The association with axon part was markedly lower after correction for worry scores (uncorrected, $P=5.26 \times 10^{-8}$; corrected for depressed affect, $P=2.42 \times 10^{-6}$; corrected for worry, $P=0.0013$ ), suggesting that the involvement of axon part in neuroticism originates predominantly from the worry component.

\section{Genetic correlations}

To examine the genetic correlational pattern of neuroticism and to compare it to the patterns observed for depression, depressed affect, and worry, we used LDSC ${ }^{116,204}$ to calculate genetic correlations with 35 traits for which large-scale GWAS summary statistics were available (Methods and Supplementary Table 36). We observed 11 Bonferroni-corrected significant genetic correlations between neuroticism and other traits $\left(\alpha=0.05 /(4 \times 35) ; P<3.6 \times 10^{-4}\right)$ (Fig. 3.4 and Supplementary Table 37), which covered previously reported psychiatric traits $\left(r_{\mathrm{g}}\right.$ range: 0.20 to 0.82$)$ and subjective well-being $\left(r_{\mathrm{g}}=-0.68\right)$. These correlations were supported by enrichment of genes associated with neuroticism in sets of genes that had previously been implicated in psychiatric traits (Supplementary Table 38). The $r_{\mathrm{g}}$ values of depression and depressed affect strongly mirrored each other (the correlation between their $r_{\mathrm{g}}$ values was $r=0.98$; Supplementary Note), which validated the depressed affect cluster. The correlational patterns for depressed affect and worry were markedly different (for example, anorexia nervosa, schizophrenia, and ever-smoker) and sometimes in opposite directions (for example, body mass index (BMI)). The genetic correlations of the full neuroticism trait seemed to be a mix of the genetic signal of both clusters, with neuroticism's $r_{\mathrm{g}}$ values generally in between the cluster-specific $r_{\mathrm{g}}$ values. 


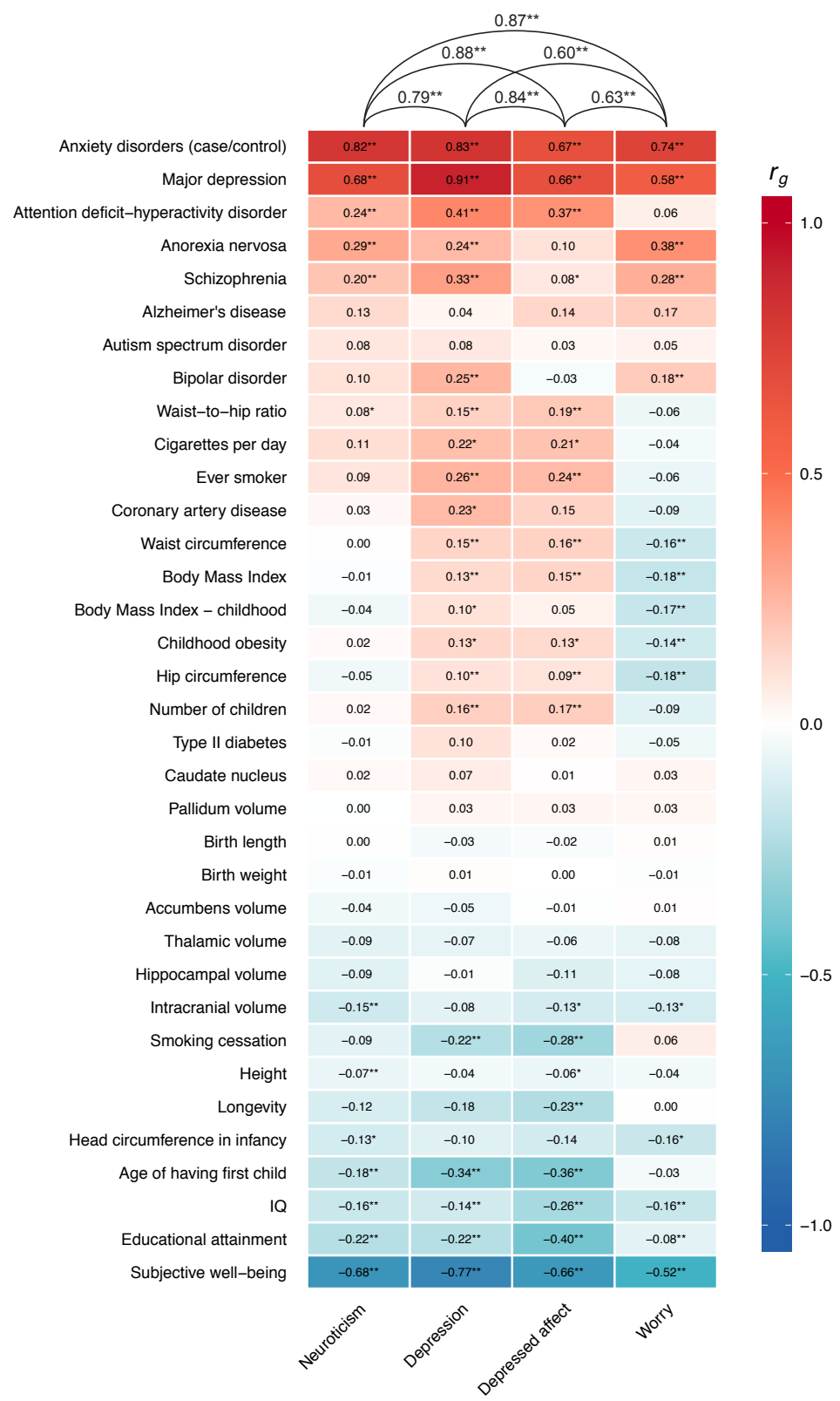

Figure 3.4. Genetic correlations between neuroticism and other traits.

Genetic correlations of neuroticism, depression, depressed affect and worry with various traits and diseases. LD Score regression (Methods) tested genome-wide SNP associations for the neuroticism score against previously published results for 35 neuropsychiatric outcomes, anthropometric and health-related traits, and brain morphology (Supplementary Table 36-37). Genetic correlations among neuroticism, depression, depressed affect and worry are displayed in the top part of this 
figure. Red and blue indicate positive and negative genetic correlations, whereas hue indicates the strength of the genetic correlations. Sample sizes for the traits in this figure are presented in Supplementary Table 36).

$* P<0.01 ; * *$ Bonferroni corrected $P$ value threshold $\left(P<3.6 \times 10^{-4}\right)$

\section{Mendelian randomization}

To investigate whether these genetic correlations reflected directional effects, we performed Mendelian randomization (MR) analysis using the GSMR package ${ }^{205}$ (Methods). Among other things, we observed unidirectional effects of BMI on depression and depressed affect ( $b_{x y}=0.061, P=4.96 \times 10^{-12}$ and $b_{x y}=0.049, P=5.35 \times 10^{-6}$, respectively) and bidirectional associations between neuroticism and depression, as well as between all four main traits and subjective well-being, cognition, and several psychiatric disorders (Supplementary Table 39 and Supplementary Note).

\section{Drug-gene interactions}

We aimed to identify gene-drug interactions (using the Drug Gene Interaction database (DGIdb) $^{206,207}$; Methods) of genes identified for each of the four traits, and we observed a large number of potential targets for pharmacotherapeutic intervention that were either shared between traits or distinct for each phenotype (Supplementary Fig. 17, Supplementary Tables 40 and 41, and Supplementary Note).

\subsection{Discussion}

In conclusion, we identified 124 new genetic loci for neuroticism (73 taking into account a simultaneously conducted study by Luciano et al. ${ }^{208}$; Supplementary Table 42 and Supplementary Note). Extensive functional annotations highlighted several genes implicated through multiple routes. We demonstrated the involvement of specific neuronal cell types and three independently associated genetic pathways, and we established the genetic multidimensionality of the neuroticism phenotype and its link with depression. The current study provides new leads and testable functional hypotheses for unraveling the neurobiology of neuroticism, its subtypes, and its genetically associated traits. 


\subsection{Methods}

\section{Samples}

UK Biobank: The UKB study is a major data resource that contains genetic, as well as a wide range of phenotypic, data of $\sim 500,000$ participants aged 39-73 years at recruitment ${ }^{203}$. We used data released in July 2017, and selection (discussed below) resulted in final sample sizes of $n=372,903$ and $n=362,696$ individuals for neuroticism and depression, respectively (Supplementary Note). The UKB received ethical approval from the National Research Ethics Service Committee North West-Haydock (reference 11/NW/0382), and all study procedures were performed in accordance with the World Medical Association for medical research. The current study was conducted under UKB application 16406.

23andMe: 23 andMe, Inc. is a large personal genomics company that provides genotype and healthrelated information to customers. For the neuroticism and depression meta-analyses, we used neuroticism and depression GWAS summary statistics, respectively, from a subset of 23andMe research participants (neuroticism: $\mathrm{N}=59,206$; depression: $\mathrm{N}=307,354$ ), described in more detail elsewhere ${ }^{139,191}$. All included participants provided informed consent and were of European ancestry, and related individuals were excluded. Online data collection procedures were approved by the Ethical \& Independent Review Services (E\&I Review), an AAHRPP-accredited private institutional review board (see URLs).

Genetics of Personality Consortium: The Genetics of Personality Consortium (GCP) is a large body of cooperation concerning GWAS on personality. We used summary statistics of neuroticism from the first GPC personality meta-analysis (GPC1, see URLs) ${ }^{129}$, on 10 discovery cohorts (SardiNIA, NTR/NESDA, ERF, SAGE, HBCS, NAG, IRPG, QIMR, LBC1936, BLSA, EGPUT), including in total $\mathrm{N}=17,375$ participants of European descent. All included studies were approved by local ethic committees, and informed consent was obtained from all participants.

Psychiatric Genetics Consortium: The Psychiatric Genetics Consortium (PGC) unites investigators worldwide to conduct genetic meta- and mega-analyses for psychiatric disorders. We used summary statistics from the latest published PGC meta-analysis on depression (see URLs) ${ }^{37}$, which included data from 8 cohorts (Bonn/Mannheim, GAIN, GenRED, GSK, MDD2000, MPIP, RADIANT, STAR*D), covering N=18,759 participants of European descent. All included studies were approved by local ethic committees, and informed consent was obtained from all participants.

\section{Phenotype assessment - Neuroticism}

UK Biobank: Neuroticism was measured with 12 dichotomous (yes/no) items of the Eysenck Personality Questionnaire Revised Short Form (EPQ-RS ${ }^{88}$, using a touchscreen-questionnaire at 
the UKB assessment centers (Supplementary Note). Participants with valid responses to $<10$ items were excluded from analyses. A weighted neuroticism sum-score was calculated by adding up individual valid item responses, and dividing that sum by the total number of valid responses. In addition, we constructed two scores based on subsets of genetically homogeneous neuroticism items, as established previously ${ }^{192}$ through hierarchical clustering analysis of the genetic correlations between the 12 neuroticism items (see Supplementary Note). Specifically, scores on 4 EPQ-RS items (i.e., "Do you often feel lonely?", "Do you ever feel 'just miserable' for no reason?", "Does your mood often go up and down?", and "Do you often feel 'fed-up'?") were summed to obtain scores for the cluster depressed affect. Similarly, scores on 4 other EPQ-RS items (i.e., "Are you a worried?", "Do you suffer from nerves?", "Would you call yourself a nervous person?", and "Would you call yourself tense or highly strung") were summed to obtain scores for the cluster worry. In the item-cluster analyses, only participants with complete scores on all 4 items were included, resulting in $\mathrm{N}=357,957$ and $\mathrm{N}=348,219$ for depressed affect and worry, respectively.

23andMe: Neuroticism was operationalized as of the sum of 8 neuroticism items (5-point Likert scale; 'Disagree strongly' to 'Agree strongly') from the Big Five Inventory $\left(\mathrm{BFI}^{209,210}\right)$, as obtained in an online survey. Only participants with valid responses to all items were included in the analyses (Supplementary Note).

Genetics of Personality Consortium: All 10 cohorts included in the first meta-analysis of the GPC used sums of the scores on 12 items (5-point Likert scale; 'Strongly disagree' to 'Strongly agree') of the NEO-FFI ${ }^{211}$ to measure neuroticism. If $<4$ item scores were missing, data on invalid items were imputed by taking an individual's average score on valid items. Participants were excluded from analyses if they had invalid scores on $>3$ items $^{129}$ (Supplementary Note).

\section{Phenotype assessment - Depression}

UK Biobank: Depression was operationalized by adding up the scores on two continuous items ("Over the past two weeks, how often have you felt down, depressed or hopeless?", "Over the past two weeks, how often have you had little interest or pleasure in doing things?"; both evaluated on a 4-point Likert scale; 'Not at all' to 'Nearly every day'), resulting in a continuous depression score (as used previously ${ }^{141}$ ). Only participants with scores on both items were included in the analyses, resulting in N=362,696 (Supplementary Note).

23andMe: This concerns a case-control sample. Four self-report survey items were used to determine case-control status. Cases were defined as replying affirmatively to at least one of these questions, and not replying negatively to previous ones. Controls replied negatively to at least one of the questions, and did not report being diagnosed with depression on previous ones (Supplementary Note). 
Psychiatric Genetics Consortium: This concerns a case-control sample. Cases had a DSM-IV lifetime (sometimes (early onset) recurrent) major depressive disorder (MDD) diagnosis, either established through structured diagnostic interviews or clinician-administered DSM-IV checklists. Most cases were ascertained from clinical sources, while controls were randomly selected from population resources and screened for lifetime history of $\mathrm{MDD}^{37}$ (Supplementary Note).

\section{Genotyping and imputation}

UK Biobank - neuroticism: We used genotype data released by the UKB in July 2017. The genotype data collection and processing are described in detail by the responsible UKB group ${ }^{193}$. In short, 489,212 individuals were genotyped on two customized SNP arrays (the UK BiLEVE Axiom array $(\mathrm{n}=50,520)$ and UK Biobank Axiom array $(\mathrm{n}=438,692))$, covering 812,428 unique genetic markers ( $95 \%$ overlap in SNP content). After quality control procedures ${ }^{193}, 488,377$ individuals and 805,426 genotypes remained. Genotypes were phased and imputed by the coordinating team to approximately 96 million genotypes using a combined refence panel including the Haplotype Reference Consortium and the UK10K haplotype panel. Imputed and quality-controlled genotype data was available for 487,422 individuals and 92,693,895 genetic variants. As recommended by the UKB team, variants imputed from the UK10K reference panel were removed from the analyses due to technical errors in the imputation process.

In our analyses, only individuals from European descent (based on genetic principal components) were included. Therefore, principal components from the 1000 Genomes reference populations ${ }^{212}$ were projected onto the called genotypes available in UK Biobank. Subjects were identified as European if their projected principal component score was closest (based on the Mahalanobis distance) to the average score of the European 1000 Genomes sample ${ }^{213}$. European subjects with a Mahalanobis distance $>6$ S.D. were excluded. In addition, participants were excluded based on withdrawn consent, UKB provided relatedness (subjects with most inferred relatives, $3^{\text {rd }}$ degree or closer, were removed until no related subjects were present), discordant sex, sex aneuploidy. After selecting individuals based on available neuroticism sum-score and active consent for participation, 372,903 individuals remained for the analyses.

To correct for population-stratification, 30 principal components were calculated on the subset of QC-ed unrelated European subjects based on 145,432 independent $\left(r^{2}<0.1\right)$ SNPs with $\mathrm{MAF}>0.01$ and INFO $=1$ using FlashPC $2{ }^{177}$. Subsequently, imputed variants were converted to hard call using a certainty threshold of 0.9. Multi-allelic SNPs, indels, and SNPs without unique rs id were excluded, as well as SNPs with a low imputation score (INFO score $<0.9$ ), low minor allele frequency $(\mathrm{MAF}<0.0001)$ and high missingness $(>0.05)$. This resulted in a total of $10,847,151$ SNPs used for downstream analysis.

UK Biobank - depression: Similar genotyping/imputation/filtering procedures as described above for the UKB neuroticism GWAS were followed for the UKB depression GWAS, resulting in $\mathrm{N}=362,696$. 


\section{Genome-wide association analyses}

UK Biobank - neuroticism: Genome-wide association analyses were performed in PLINK ${ }^{175,176}$, using a linear regression model of additive allelic effects with age, sex, townsend deprivation index, genotype array, and 10 genetic European-based principal components as covariates (Supplementary Note).

UK Biobank - depression, depressed affect, worry: The settings, covariates, and exclusion criteria for the UKB depression, UKB depressed affect, and UKB worry GWAS were the same as described above for UKB neuroticism GWAS, with 10,847,151 SNPs remaining after all exclusion steps (Supplementary Note).

Other samples: Summary statistics were used for $23 \mathrm{andMe}$, GPC and PGC. Details on the genomewide association analyses of these samples can be found elsewhere (23andMe neuroticism ${ }^{191}$; 23andMe depression ${ }^{139}$; GPC neuroticism ${ }^{129}$; PGC depression ${ }^{37}$ ).

\section{Meta-analysis}

To maximize the statistical power to detect associated genetic variants of small effect, we conducted meta-analyses for both neuroticism and depression ${ }^{195}$ (Supplementary Note). All metaanalyses were performed in METAL ${ }^{179}$.

Neuroticism: The meta-analysis of the neuroticism GWAS in UKB, 23andMe, and GPC was performed on the $P$ value of each SNP using a sample size-weighted fixed-effects analysis. Bonferroni correction was applied to correct for multiple testing. The genetic signal correlated strongly between the three samples ( $r_{g}$ range: $0.83-1.07$; Supplementary Table 1), supporting the decision to meta-analyze.

Depression: As the UKB GWAS concerned a continuous operationalization of the depression phenotype, while 23andMe and PGC used case-control phenotypes, the odds ratio from the 23andMe and PGC summary statistics were converted to log odds, reflecting the direction of the effect. The meta-analysis was then performed on the $P$ value of each SNP using a sample size weighted fixed-effects analysis. Bonferroni correction was applied to correct for multiple testing. Genetic correlations between the three samples were moderate to strong ( $r_{g}$ range: $0.61-0.80$; Supplementary Table 22).

\section{Genomic risk loci and functional annotation}

Functional annotation was performed using $\mathrm{FUMA}^{17}$ (see URLs), an online platform for functional mapping of genetic variants. We first defined independent significant SNPS which have a genomewide significant $P$ value $\left(5 \times 10^{-8}\right)$ and are independent at $r^{2}<0.6$. A subset of these independent 
significant SNPs, that were independent from each other at $r^{2}<0.1$, was marked as lead SNPs (based on LD information from UK Biobank genotypes; see Supplementary Note for a more detailed explanation). Subsequently, genomic risk loci were defined by merging lead SNPs that physically overlapped or for which LD blocks were less than $250 \mathrm{~kb}$ apart. Note that when analyzing multiple phenotypes, as in the current study, a locus may be discovered for different phenotypes, whilst different lead SNPs are identified.

All SNPs in the meta-analysis results that were in $\operatorname{LD}\left(r^{2}>0.6\right)$ with one of the independent significant $S N P S$, had a $P$ value lower than $1.0 \times 10^{-5}$ and minor allele frequency (MAF) $>0.0001$ were selected for annotation. The rationale behind this inclusive approach is that the most significant SNP in the locus is not necessarily the causal SNP, but may be in LD with the causal SNP. We thus annotated all SNPs in LD with the most significant SNP to get insight into the possible biological reasons for observing a statistical association. We note that liberalizing the $r^{2}$ and $P$ value thresholds can dilute the functional annotation results, while more stringent thresholds may result in exclusion of possibly interesting functional variants. Functional consequences for these SNPs were obtained by performing ANNOVAR ${ }^{214}$ gene-based annotation using Ensembl genes. In addition, CADD scores (indicating the deleteriousness of a SNP, with scores $>12.37$ seen as likely deleterious ${ }^{215}$ ) and RegulomeDB scores $^{181}$ (where a higher probability of having a regulatory function is indicated by lower scores) were annotated to SNPs by matching chromosome, position, reference and alternative alleles. CADD scores integrate a number of diverse annotations into a single measure that correlates with pathogenicity, disease severity and experimentally measured regulatory effects and complex trait associations ${ }^{215}$.

\section{Gene-mapping}

SNPs in genomic risk loci that were GWS or were in LD $\left(r^{2}>0.6\right)$ with one of the independent significant SNPs were mapped to genes in $\mathrm{FUMA}^{216}$ using either of three strategies.

First, positional mapping uses the physical distances (i.e., within $10 \mathrm{~kb}$ window) from known protein coding genes in the human reference assembly (GRCh37/hg19) to map SNPs to genes. The second strategy, eQTL mapping, uses information from 3 data repositories (GTEx, Blood eQTL browser BIOS QTL browser), and maps SNPs to genes based on a significant eQTL association (i.e. the expression of that gene is associated with allelic variation at the SNP). eQTL mapping is based on cis-eQTLs which can map SNPs to genes up to $1 \mathrm{Mb}$ apart. FUMA applied a false discovery rate (FDR) of 0.05 to define significant eQTL associations. Third, chromatin interaction mapping mapped SNPs to genes based on a significant chromatin interaction between a genomic region in a risk locus and promoter regions of genes (250bp up- and 500bp downstream of transcription start site (TSS)). This type of mapping does not have a distance boundary (as in eQTL mapping), and may therefore involve long-range interactions. Currently, FUMA contains Hi-C data of 14 tissue types from the study of Schmitt et al. (2016) 217 . Notably, as chromatin interactions are usually defined in a certain resolution (in the current study; $40 \mathrm{~kb}$ ), an interacting region may span several genes. Hence, this method would map all SNPs within these regions to genes in the 
corresponding interaction region. By integrating predicted enhancers and promoters in 111 tissue/cell types from the Roadmap Epigenomics Project ${ }^{218}$ we aimed to prioritize candidate genes from chromatin interaction mapping. Using this information FUMA selected chromatin interactions for which one region involved in the interaction overlapped with predicted enhancers and the other with predicted promoters in $250 \mathrm{bp}$ upstream and 500bp downstream of TSS of a gene. Similar to eQTL mapping, we used a FDR of $1 \times 10^{-5}$ to define significant interactions.

\section{Gene-based analysis}

GWGAS can identify genes in which multiple SNPs show moderate association to the phenotype of interest without reaching the stringent genome-wide significance level. At the same time, because GWGAS takes all SNPs within a gene into account, a gene harboring a genome-wide significant SNP may not be implicated by GWGAS analyses when multiple other SNPs within that gene show only very weak association signal. The $P$ values from the SNP-based GWAS metaanalyses for neuroticism and depression, and the GWAS for depressed affect and worry, were used as input for the GWGAS in MAGMA (see URLs) ${ }^{150}$, and all 19,427 protein-coding genes from the NCBI 37.3 gene definitions were used. We annotated all of the SNPs in our genome-wide association (meta-)analyses to these genes, resulting in 18,187, 18,187, 18,182, and 18,182 genes that were represented by at least one SNP in the neuroticism meta-analysis, the depression metaanalysis, the depressed affect GWAS, and the worry GWAS, respectively. We included a window around each gene of $2 \mathrm{~kb}$ before the TSS and $1 \mathrm{~kb}$ after the transcription stop site. Gene association tests were performed, taking into account the LD between SNPs, and a stringent Bonferroni correction was applied to correct for multiple testing $(0.05 /$ number of genes tested: $P<2.75 \times 10^{-6}$ ).

\section{Gene-set analysis}

We used MAGMA ${ }^{150}$ to test for association of predefined gene sets with neuroticism, depression, depressed affect, and worry. A total of 7,246 gene sets were derived from several resources, including BioCarta, KEGG, Reactome ${ }^{219}$, and GO. All gene sets were obtained from MSigDB version 6.0 (see URLs). Additionally, we performed gene set analysis on 53 tissue expression profiles obtained from the GTEx portal (see URLs) and on 24 cell-type-specific expression profiles.

For all gene sets, we computed competitive $P$ values, which result from testing whether the combined effect of genes in a gene set is significantly larger than the combined effect of the same number of randomly selected genes (in contrast to testing against the null hypothesis of no effect; self-contained test). Here we only report Bonferroni-corrected $\left(\alpha=0.05 / 7,323=6.83 \times 10^{-6}\right)$ competitive $P$ values, which were more conservative than the self-contained $P$ values. 


\section{Cell type specific expression analysis}

Definition and calculation of gene sets for cell type specific expression is described in detail elsewhere 220,221 . Briefly, brain cell type expression data was drawn from scRNAseq data from mouse brain ${ }^{221}$. For each gene, the value for each cell type was calculated by dividing the mean Unique Molecular Identifier (UMI) counts for the given cell type by the summed mean UMI counts across all cell types ${ }^{221}$. MAGMA ${ }^{150}$ was used to calculate associations between gene-wise $P$ values from the meta-analysis and cell type specific gene expression. Genes were grouped into 40 equal bins by specificity of expression, and subsequently bin-membership was regressed on gene-wise association with neuroticism in the meta-analysis. Results were deemed significant if the association $P$ values were smaller than the relevant Bonferroni threshold.

\section{Conditional gene-set analyses}

Conditional gene-set analyses were performed using MAGMA ${ }^{150}$ to determine which tissue expression levels and MsigDB gene-sets represent independent associations. In these regressionbased analyses, the effect of a gene-set (or tissue expression) of interest is conditioned on the effects of another gene-sets (or tissue expressions) to correct the association of the tested gene-set for any effect it shares with the conditioned-on gene-sets.

For the MSigDB gene-sets we conducted two series of conditional analyses. First, we performed a forward selection on the initially significant gene-sets, in each step selecting the most strongly associated gene-set after conditioning on all already selected gene-sets. (Supplementary

Table 19). Second, to test whether the association of gene-sets to neuroticism is primarily driven by association signal of one specific subcluster, we also reran the GO gene-set analyses conditioning on the gene $z$-scores of depressed affect or worry, respectively (Supplementary Table 35). If the gene-set association decreases after conditioning on one cluster but not, or less so, when conditioning on the other, then this suggests that neuroticism's association to that geneset is primarily driven by the genetic effects of the first, and not the second, item cluster.

\section{Genetic correlations}

Genetic correlations $\left(r_{g}\right)$ were computed using LD Score regression ${ }^{116,146}$ (see URLs). The significance of the genetic correlations of neuroticism, depression, depressed affect and worry with 35 behavioral, social and (mental) health phenotypes for which summary statistics were available was determined by correcting for multiple testing through a stringent Bonferroni corrected threshold of $\left.P<0.05 /(4 \times 35)=3.6 \times 10^{-4}\right)$.

\section{Mendelian randomization analyses}

We performed Mendelian randomization (MR) to test whether genetic correlations could be explained by directional effects between traits. Generalised summary-data based Mendelian 
randomization (GSMR ${ }^{222}$ ) was used for MR analysis: a summary statistics-based MR method that uses independent genome-wide significant variants as instrumental variables. Causal associations were tested between the four traits, and the 21 traits that showed significant genetic correlations $\left(r_{g}\right)$ in LD Score regression analysis with at least one of the four traits. To test for uni- and bidirectional effects, we performed both forward and reverse GSMR analysis (i.e., using the four GWAS traits either as predictor or as outcome). Associations were Bonferroni corrected for multiple testing $\left.P<0.05 /(21 * 4 * 2)=2.98 \times 10^{-4}\right)$.

\section{Partitioned heritability}

To investigate the relative contribution to the overall SNP-based heritability annotated to 22 specific genomic categories, we partitioned SNP heritability by binary annotations using stratified LD Score regression ${ }^{223}$. Information about binary SNP annotations were obtained from the LD Score website (see URLs). Enrichment results reflect the X-fold increase in $h^{2}$ proportional to the number of SNPs (e.g., enrichment=13.79 for SNPs in conserved regions implies that a 13,79-fold increase in $h^{2}$ is carried by SNPs in these regions, corrected for the proportion of SNPs in these regions compared to all tested SNPs).

\section{Gene drug targets}

We aimed to identify potential druggable targets by performing lookup of implicated genes (by one of the gene-mapping strategies) in the drug-gene interaction database (DGIdb ${ }^{224,225}$, version 3.0: see URLs). The DGIdb database contains mined data from several resources, and provides a comprehensive overview of the druggability of gene targets. First, we searched 20 drug-gene databases for interactions with existing medicines based on 48 known interaction types with genes that were implicated in each of the four phenotypes. Filtering was performed based on known interaction types, and interactions with FDA-approved pharmaceutical compounds. Second, to identify genes that may form targets for novel therapies in addition to existing medicines, we searched for potential gene druggability of gene targets and performed an additional search in 10 DGIdb databases containing information about gene targetability.

\section{Polygenic risk scoring}

To test the predictive accuracy $\left(\Delta R^{2}\right)$ of the meta-analytic results for neuroticism, we calculated a polygenic risk score (PGS) based on the SNP effect sizes of the current analysis. For independent samples, we used three hold-out samples; we removed 3,000 individuals from the discovery sample (UKB only, as we only had access to individual-level data from this sample) and re-ran the genomewide analyses. We repeated this process three times, to create three randomly drawn, independent hold-out samples. Next, we calculated a PGS on the individuals in each of the 3 holdout samples. PGS were calculated using LDpred ${ }^{226}$ and PRSice ${ }^{227}$ (clumping followed by $P$ value thresholding). 
For LDpred, PGS were calculated based on different LDpred priors $\left(P_{L D p r e d}=0.01,0.05,0.1\right.$, $0.5,1$ and infinitesimal). The explained variance $\left(R^{2}\right)$ was derived from the linear model, using the neuroticism summary score as the outcome, while correcting for age, gender, array, townsend deprivation index and genetic principal components. 


\subsection{Supplementary methods}

\section{Phenotype measurement}

\section{UK Biobank - Neuroticism, depressed affect, and worry}

To operationalize neuroticism, we used raw item level data obtained from the UK Biobank study (application number 16406). Neuroticism was measured with 12 dichotomous ("yes"/"no") items of the Eysenck Personality Questionnaire Revised Short Form (EPQ-RS ${ }^{88}$ ). All 12 items are displayed in the table below. The EPQ-RS neuroticism scale has been shown to have reliability $>0.8^{88}$ and correlates strongly $(r=0.85)$ with the neuroticism score from the NEO Five-Factor Inventory (NEO-FFI) ${ }^{228}$, which was predominantly used in the GPC1 sample.

During the first visit of the UK Biobank data collection (2006-2010) the EPQ-RS neuroticism scale was included in the touch-screen assessment held at the UKB assessment centers. Participants with valid responses to $<10$ items were excluded from our analyses. A weighted neuroticism sumscore was calculated by adding up individual valid item responses, and dividing that sum by the total number of valid responses. Higher sum-scores indicate higher levels of neuroticism.

In total, $\mathrm{N}=372,903$ participants remained after excluding participants with $>2$ missing responses, who did not have valid genetic data, or were not of European descent. The distribution of item-level missingness is shown in Supplementary Fig. 2a. The distribution of the sum-score, after excluding participants, is shown in Supplementary Fig. 2b. The mean age in the resulting sample was 56.7 years $(\mathrm{SD}=8.0)$, and $54.0 \%$ was female. The mean score was $0.35(\mathrm{SD}=0.27)$, and was higher in females compared to males (Cohen's $d=0.30$ ).

Scores on 4 EPQ-RS items (top four in the table below) were summed to obtain scores for the cluster depressed affect. Similarly, scores on 4 other EPQ-RS items (bottom 4)) were summed to obtain scores for the cluster worry. For a detailed discussion of the clusters we refer to Nagel et al. Briefly, following item-level GWAS, genetic correlations between the 12 EPQ neuroticism items were computed. Subsequently, hierarchical clustering analysis was performed on the genetic correlations between these 12 items, revealing two clear clusters, that replicated in a second, independent sample. In the item-cluster analyses, only participants with complete scores on all 4 items were included, resulting in $\mathrm{N}=357,957$ and $\mathrm{N}=348,219$ for depressed affect and worry, respectively. The mean score for the depressed affect cluster was 1.45 ( $\mathrm{SD}=1.42)$, and was higher in females compared to males (Cohen's $d=0.20$ ). The mean score for the worry cluster was 1.16 ( $\mathrm{SD}=1.25$ ), and was higher in females compared to males (Cohen's $d=0.22$ ). Age, sex, Townsend deprivation index, genotyping array and the first 10 European-based genetic principal components were included in the analysis as covariates. 
Individual items of the EPQ-RS neuroticism questionnaire used to measure neuroticism in the UK Biobank sample ( $\mathrm{N}=372,903)$, and missingness and endorsement rates

\begin{tabular}{|c|c|c|c|c|}
\hline \multirow[b]{2}{*}{ Item (UKB field ID) } & \multicolumn{4}{|c|}{ Endorsement rate $(\%)$} \\
\hline & $\mathrm{N}_{\text {miss }}$ & Total & Male & Female \\
\hline Does your mood often go up and down? (1920) & 5,340 & 44.68 & 42.27 & 46.73 \\
\hline $\begin{array}{l}\text { Do you ever feel 'just miserable' for no reason? } \\
\text { (1930) }\end{array}$ & 3,370 & 42.57 & 35.35 & 48.72 \\
\hline Do you often feel 'fed-up'? (1960) & 4,111 & 40.11 & 37.49 & 42.34 \\
\hline Do you often feel lonely? (2020) & 3,111 & 17.89 & 14.67 & 20.63 \\
\hline Are you an irritable person? (1940) & 11,200 & 28.25 & 30.84 & 26.04 \\
\hline Are your feelings easily hurt? (1950) & 6,715 & 55.20 & 44.94 & 63.95 \\
\hline $\begin{array}{l}\text { Do you worry too long after an embarrassing } \\
\text { experience? (2000) }\end{array}$ & 10,452 & 47.44 & 39.87 & 53.95 \\
\hline Are you often troubled by feelings of guilt? (2030) & 5,235 & 28.50 & 21.77 & 34.26 \\
\hline Would you call yourself a nervous person? (1970) & 5,504 & 23.47 & 19.44 & 26.94 \\
\hline Are you a worrier? (1980) & 5,956 & 56.24 & 47.45 & 63.74 \\
\hline $\begin{array}{l}\text { Would you call yourself tense or 'highly strung'? } \\
\text { (1990) }\end{array}$ & 6,998 & 17.55 & 15.21 & 19.56 \\
\hline \multirow[t]{2}{*}{ Do you suffer from 'nerves'? (2010) } & 8,641 & 21.18 & 21.43 & 20.97 \\
\hline & \multicolumn{3}{|c|}{ Mean (SD) } & \\
\hline Neuroticism sum-score & - & $\begin{array}{c}0.35 \\
(0.27)\end{array}$ & $\begin{array}{c}0.31 \\
(0.27)\end{array}$ & $\begin{array}{c}0.39 \\
(0.27)\end{array}$ \\
\hline Depressed affect & 14,946 & $\begin{array}{c}1.45 \\
(1.42)\end{array}$ & $\begin{array}{c}1.29 \\
(1.38)\end{array}$ & $\begin{array}{c}1.58 \\
(1.45)\end{array}$ \\
\hline Worry & 24,684 & $\begin{array}{c}1.16 \\
(1.25)\end{array}$ & $\begin{array}{c}1.01 \\
(1.22)\end{array}$ & $\begin{array}{c}1.28 \\
(1.27)\end{array}$ \\
\hline \multicolumn{5}{|c|}{$\begin{array}{l}\text { Note: } \\
\text { The top } 4 \text { items belong to the depressed affect cluster, whereas the bottom } 4 \text { items belong to the } \\
\text { worry cluster. }\end{array}$} \\
\hline
\end{tabular}

\section{3andMe - Neuroticism}

For the neuroticism meta-analysis, we used neuroticism GWAS summary statistics from a subset of 23 andMe participants $(\mathrm{N}=59,206)$, described in more detail elsewhere ${ }^{191}$.

In the 23andMe sample, neuroticism was measured as a quantitative trait using an adapted version of the Big Five Inventory $\left(\mathrm{BFI}^{209}\right)$. Participants were asked to indicate on a 5-point Likert scale (ranging from 'strongly disagree' to 'strongly agree') whether the 8 statements shown below applied to them. The BFI facet scales (including the neuroticism scale) have been shown to have good reliability and agree well with self-reports on the Revised NEO Personality inventory ${ }^{210}$.

1. I am someone who is depressed, often feels sad

2. I am someone who is relaxed, handles stress well (R)

3. I am someone who can be tense

4. I am someone who worries a lot

5. I am someone who is emotionally stable, not easily upset (R) 
6. I am someone who can be moody

7. I am someone who remains calm in tense situations (R)

8. I am someone who gets nervous easily

Note: items were evaluated on a 5-point Likert scale ('Disagree strongly' to 'Agree strongly'). $\mathrm{R}=$ Reverse-coded item.

The final sample size, consisting of participants with valid BFI scores and valid genetic data, who were genetically unrelated and of $>97 \%$ European ancestry, consisted of $\mathrm{N}=59,206$ participants, $49 \%$ female. For our meta-analysis, we used GWA summary statistics. Item-level information was not available from 23 andme. Age, sex and the first 5 genetic principal components were included as covariates in the GWAS analyses.

\section{Genetics of Personality Consortium - Neuroticism}

We used summary statistics of neuroticism from the first GPC personality meta-analysis (GPC1, http://www.tweelingenregister.org/GPC/) ${ }^{129}$, on 10 discovery cohorts (SardiNIA (Italy), NTR/NESDA (the Netherlands), ERF (the Netherlands), SAGE (United States of America), HBCS (Finland), NAG/IRPG (Australia), QIMR(Australia), LBC1936 (United Kingdom), BLSA (United States of America), and EGPUT (Estonia)), including in total N=17,375 participants of European descent. All samples are described in detail in the original publication ${ }^{129}$.

In most GPC1 samples, neuroticism was measured as a quantitative trait using the sums of scores on 12 items (5-point Likert scale; 'Strongly disagree' to 'Strongly agree'; listed below) of the NEO-FFI' ${ }^{211}$ (otherwise, the related instrument NEO-PI-R was used).

1. I'm not a worrier (R)

2. I often feel less than others

3. When I'm under pressure, sometimes I can't handle it

4. I rarely feel lonely or sad (R)

5. I often feel tense or nervous

6. Sometimes I feel completely worthless

7. I rarely feel anxious (R)

8. I often get mad about the way people treat me

9. When things go wrong, I often get discouraged and feel like giving up

10. I'm rarely sad or depressed (R)

11. I often feel helpless and then want someone to solve my problems

12. Sometimes I'm so embarrassed I don't know where to put it

Note: items were evaluated on a 5-point Likert scale ('Strongly disagree' to 'Strongly agree'). $\mathrm{R}=$ Reverse-coded item. 
If $<4$ item scores were missing, data on invalid items were imputed by taking an individual's average score on valid items. Participants were excluded from analyses if they had invalid scores on $>3$ items $^{129}$.

Sample-specific information on the $\%$ females, mean age (distinguished by sex), and mean scale scores (distinguished by sex) is given in detail in the original publication ${ }^{129}$. In the original analyses, age and sex were included as covariates in all analyses, and population stratification effects were corrected by applying genomic control in each sample before meta-analysis.

\section{UK Biobank - Depression}

To operationalize depression, we used raw data on two depression items obtained from the UK Biobank study (application number 16406). Depression was operationalized by adding up the scores on two continuous items (both evaluated on a 4-point Likert scale; 'Not at all' to 'Nearly every day'; items are listed below), resulting in a continuous depression score (as used previously ${ }^{141}$ ). Only participants with scores on both items were included in the analyses, resulting in $\mathrm{N}=362,696$.

The distribution of the depressive symptoms operationalization is shown in Supplementary Fig. 6. The mean age in the resulting sample was 56.7 years $(\mathrm{SD}=8.0)$, and $54.0 \%$ was female. The mean score was 2.55 ( $\mathrm{SD}=1.09$ ), and was higher in females compared to males (Cohen's $d=0.06$ ). Age, sex, townsend deprivation index, genotyping array and the first 10 European-based genetic principal components were included in the analysis as covariates.

\begin{tabular}{|c|c|c|c|c|}
\hline \multirow[b]{2}{*}{ Item } & \multirow[b]{2}{*}{$N_{\text {miss }}$} & \multicolumn{3}{|c|}{ Mean (SD) } \\
\hline & & Total & Male & Female \\
\hline $\begin{array}{l}\text { Over the past two weeks, how often have you felt } \\
\text { down, depressed or hopeless? (UKB field ID: 2050) }\end{array}$ & 0 & $\begin{array}{c}1.29 \\
(0.61)\end{array}$ & $\begin{array}{c}1.25 \\
(0.57)\end{array}$ & $\begin{array}{c}1.32 \\
(0.63)\end{array}$ \\
\hline $\begin{array}{l}\text { Over the past two weeks, how often have you had little } \\
\text { interest or pleasure in doing things? (UKB field ID: 2060) }\end{array}$ & 0 & $\begin{array}{c}1.26 \\
(0.60)\end{array}$ & $\begin{array}{l}1.27 \\
(0.60)\end{array}$ & $\begin{array}{c}1.26 \\
(0.60)\end{array}$ \\
\hline Depressive symptoms (SUM) & 0 & $\begin{array}{c}2.56 \\
(1.09)\end{array}$ & $\begin{array}{c}2.52 \\
(1.05)\end{array}$ & $\begin{array}{c}2.59 \\
(1.11)\end{array}$ \\
\hline
\end{tabular}

We note that the UKB data base does not contain a true clinical case-control measure of depression. Multiple researchers (e.g., Howard et al. ${ }^{229}$, Wray et al. ${ }^{230}$ ) have created case-control phenotypes by combining answers to multiple questions. We note that this often leads to a (much) lower sample size than our depression operationalization. For instance, Howard et al. studied three different case-control phenotypes for depression: based on ICD-10 $\left(\mathrm{N}_{\text {cases }}=8,276, \mathrm{~N}_{\text {controls }}=217,584\right.$, $\left.\mathrm{N}_{\text {total }}=225,860\right)$, a "probable depression" phenotype $\left(\mathrm{N}_{\text {cases }}=30,603, \quad \mathrm{~N}_{\text {controls }}=143,916\right.$, $\left.\mathrm{N}_{\text {total }}=174,519\right)$, and a "broad depression" phenotype $\left(\mathrm{N}_{\text {cases }}=113,769, \mathrm{~N}_{\text {controls }}=208,811\right.$, $\mathrm{N}_{\text {total }}=322,580$ ). Using the two items to construct a measure of depressive symptoms, we obtained a depression score for $\mathrm{N}=362,696$ subjects, which exceeds the sample size available for the various 
case-control operationalizations by a considerable 40,116 to 188,177 subjects. The genetic correlation between our meta-analysis results and results of a meta-analysis in which the "probable depression" phenotype was used, is very high $\left(r_{g}=0.930, \mathrm{SE}=0.010\right.$, see Section 3.5.2.7 below).

\section{3andMe - Depression}

For the depression meta-analysis, we used Depression GWAS summary statistics from a subset of 23andMe participants ( $\mathrm{N}=307,354)$, described in more detail elsewhere ${ }^{139}$. This concerns a casecontrol sample. Four self-report survey items were used to determine case-control status (see below; response categories: "Yes", "No", “I don't know"). Cases were defined as replying affirmatively to at least one of these questions, and not replying negatively to previous ones. Controls replied negatively to at least one of the questions, and did not report being diagnosed with depression on previous ones.

1. Have you ever been diagnosed by a doctor with any of the following psychiatric conditions? (Depression) - from: Your Medical History survey

2. Have you ever been diagnosed with clinical depression? - from: Research Snippet

3. Have you ever been diagnosed with or treated for any of the following conditions? (Depression) - from: Health Intake

4. In the last 2 years, have you been newly diagnosed with or started treatment for any of the following conditions? (Depression) - from: Health Follow-up survey

The final sample size, consisting of participants with valid case-control status for depression and valid genetic data, who were genetically unrelated and of $>97 \%$ European ancestry, consisted of $\mathrm{N}=307,354$ participants, $48 \%$ female (cases: $62 \%$ female; controls: $44 \%$ female). In our metaanalysis, we used GWA summary statistics. Item-level information was not made available. Age, sex and the first 5 genetic principal components were included as covariates in the GWAS analyses.

\section{Psychiatric Genetics Consortium - Depression}

For the depression meta-analysis, we used summary statistics from the latest published PGC metaanalysis on depression (http://www.med.unc.edu/pgc/results-and-downloads) ${ }^{37}$, which included data from 8 cohorts (Bonn/Mannheim, GAIN, GenRED, GSK, MDD2000, MPIP, RADIANT, STAR*D), covering N=18,759 participants of European descent. This concerns a case-control sample. Cases had a DSM-IV lifetime (sometimes (early onset) recurrent) major depression disorder (MDD) diagnosis, either established through structured diagnostic interviews or clinicianadministered DSM-IV checklists. Most cases were ascertained from clinical sources, while controls were randomly selected from population resources and screened for lifetime history of $\mathrm{MDD}^{37}$. Details on the clinical definition of a case are given in the original publication ${ }^{37}$. Age, sex and five principal components reflecting ancestry were used as covariates in the original meta-analysis. 


\subsection{Supplementary results}

GWAS results per cohort and genetic correlations between cohorts included in the neuroticism meta-analysis

For each of the three cohorts (UKB, 23andMe, and GPC1) an individual GWAS was run and inspected for inflation possibly due to insufficiently corrected population stratification, by inspecting the lambda inflation factor as well as the LD Score regression (LDSC) intercept. A lambda $>1$ suggest an inflation of the genetic effects which can be due to both spurious and genuine effects, and is likely to increase with sample size ${ }^{231}$. An LDSC intercept $>1$ suggests that there is spurious association, and an intercept $<1.10$ is generally considered to suggest that the signal is mostly due to genuine association effects. For the UKB cohort, the genomic inflation factor $\lambda_{\mathrm{GC}}$ computed using LDSC, was 1.60 (Supplementary Table 1), suggesting inflation in median test statistic compared to the expected distribution. The LDSC intercept of 1.03 for UKB, however, suggested that the observed inflation in genetic signal is mostly due to polygenicity and unlikely to be driven by population stratification ${ }^{196}$. Specifically, the LDSC ratio of 0.021 indicated that as much as $97.9 \%$ of the observed inflation could be ascribed to true polygenicity and large sample size. We also conducted an additional GWAS for neuroticism in the UKB cohort, with 30 instead of 10 genetic principal components as covariates, to control more rigorously for subtle population stratification than in the first analysis. The genomic inflation factor $\lambda_{\mathrm{GC}}$ remained unchanged (1.60), suggesting that the inflation of lambda observed in the original GWAS for neuroticism was not due to insufficiently controlled ancestry.

For the (much smaller) 23andMe and GPC1 samples, $\lambda_{\mathrm{GC}}$ was close to 1 (1.06 and 1.02 , respectively) indicating only minor inflation. QQ-plots of the individual cohorts (Supplementary Fig. 7) also did not suggest issues concerning population stratification.

Genetic correlations between the GWAS results from the three cohorts included in the metaanalysis (Supplementary Table 1) were calculated with LDSC. An estimated $r_{g}$ that is out of bounds ( $<-1.25$ or $>1.25$, LDSC does not limit correlations to -1 and 1$)$ usually means that one of the $h^{2}$ estimates was very close to zero. We do also note that to the best of our knowledge the statistical properties of LDSC have not been tested in the context of large discrepancies in sample sizes (one sample $>300,000$ and the other $<100,000$ ), and that there is a need for novel tools that can calculate genetic correlations based on summary statistics using current sample sizes and currently available SNP platforms.

Estimates of $r_{g}$ were high and ranged from $0.829-1.074$. As expected, the SNP-based $h^{2}$ was lowest in the smallest cohort (GPC1: $0.048, \mathrm{SE}=0.027)$, and very similar in the UKB $(0.106$, $\mathrm{SE}=0.004)$ and $23 \mathrm{andMe}$ cohorts $(0.116, \mathrm{SE}=0.016)$, as well as in the meta-analyses $\left(h^{2}=0.100\right.$, $\mathrm{SE}=0.003)$.

The $\lambda_{\mathrm{GC}}$ of the meta-analysis was 1.65 . Traditionally, an observed $\lambda_{\mathrm{GC}}$ that deviates from 1 has been interpreted as inflation in test statistics that may be due to systematic bias (such as insufficiently controlled population stratification). However, with the availability of larger sample 
sizes (e.g. $N>100,000)$ the value of $\lambda_{\mathrm{GC}}$ is expected to increase in case of high polygenicity. See Yang et al. ${ }^{196}$ who through theory, simulation and real data analysis show that in the absence of population structure and other technical artefacts, but in the presence of true polygenic inheritance, substantial genomic inflation is expected, with the size of the inflation depending on sample size, heritability, linkage disequilibrium structure and the number of causal variants.

Indeed, high values of $\lambda_{\mathrm{GC}}$ are frequently observed in recent large-sample GWAS studies for diverse and polygenic traits, including height ${ }^{45}\left(\lambda_{\mathrm{GC}}=2.7\right.$ in European ancestry studies for common markers, $\mathrm{N}=381,625), \mathrm{BMI}^{232}\left(\lambda_{\mathrm{GC}}=1.53, \mathrm{~N}=234,069\right)$, bone mineral density ${ }^{233}\left(\lambda_{\mathrm{GC}}=1.37, \mathrm{~N}\right.$ $=142,487)$, schizophrenia ${ }^{234}\left(\lambda_{\mathrm{GC}}=1.47, \mathrm{~N}=82,315\right)$, and educational attainment ${ }^{235}\left(\lambda_{\mathrm{GC}}=1.28, \mathrm{~N}\right.$ $=293,723)$ and both the UKB $\lambda_{\mathrm{GC}}$ of 1.60 and the $\lambda_{\mathrm{GC}}$ of 1.65 of the full meta-analysis thus seems to fit with what is observed in other traits for which large sample sizes are available.

However, to allow comparisons across studies of different sample sizes and interpretation of the extent of inflation we also calculated $\lambda_{1000} . \lambda_{1000}$ can be used for case-control studies (see Freedman et al., 2017 236 ) and is the expected $\lambda_{\mathrm{GC}}$ for a sample size of 1000 cases and 1000 controls given the $\lambda_{\mathrm{GC}}$ and $\mathrm{N}$ of the original study. It can be calculated as follows:

$$
\lambda_{1000}=1+\left(\lambda_{\text {obs }}-1\right) * \frac{\left(\frac{1}{N_{\text {cases }}}+\frac{1}{N_{\text {controls }}}\right)}{\frac{1}{N_{\text {cases }, 1000}}+\frac{1}{N_{\text {controls }, 1000}}}
$$

We adjusted this formula to calculate the expected $\lambda_{1000}$ in a non- case-control setting:

$$
\lambda_{1000}=1+\left(\lambda_{o b s}-1\right) * \frac{\left(\frac{1}{N_{o b s}}\right)}{\frac{1}{1000}}
$$

where $\lambda_{\text {obs }}$ and $\mathrm{N}_{\mathrm{obs}}$ are the observed $\lambda_{\mathrm{GC}}$ and the observed sample size in the original analysis.

When we use this formula to calculate the expected $\lambda$ for $\mathrm{N}=1000, \mathrm{~N}=10,000$ and $\mathrm{N}=100,000$, given our total observed sample size of $\mathrm{N}_{\mathrm{obs}}=499,484$ and $\lambda_{\mathrm{obs}}=1.652$, we obtained $\lambda_{1000}=1.001$, $\lambda_{10000}=1.015$, and $\lambda_{100000}=1.145$, respectively, showing that 1$)$ the expected $\lambda_{\mathrm{GC}}$ would be much closer to 1 if the sample size was smaller, and 2) the expected $\lambda_{\mathrm{GC}}$ increases considerably with increasing $\mathrm{N}$, as is expected for a highly polygenic trait.

Apart from the above, we also calculated $\lambda_{\mathrm{GC}}$ outside of LDSC, using an R script and basing our calculation on all SNPs (whereas LDSC only uses HapMap SNPs). We implemented the formula: $\lambda_{G C}=\operatorname{median}\left(\chi^{2}\right) / q \operatorname{chisq}(0.5,1)$. This resulted in a substantially lower value of 1.24 . This lower value is likely due to the addition of a relatively large chunk of non-HapMap SNPs and mostly rarer variants that have much smaller effect sizes, and indicates lower inflation when 
considering all SNPs. However, we believe the LDSC $\lambda_{\mathrm{GC}}$ should be used when comparing our results to other studies that also used the LDSC $\lambda_{\mathrm{GC}}$.

\section{Prediction of neuroticism in three independent hold-out samples}

To estimate the variation in neuroticism that could be explained by our GWA meta-analysis in independent samples, we re-ran our GWA meta-analysis for neuroticism three times, each time excluding a UKB hold-out sample of $\mathrm{N}=3,000$ randomly drawn individuals. We then calculated polygenic scores (PGS) using two methods: PRSice ( $P$ value thresholding and clumping) and LDpred $^{226}$. In the first hold-out sample, the PGS explained at most $3.8 \%\left(P=2.12 \times 10^{-28}\right)$ of the variance in neuroticism. In the second hold-out sample, the PGS explained at most $3.0 \%$ $\left(P=1.88 \times 10^{-22}\right)$ of the variance in neuroticism. In the third hold-out sample, the PGS explained at most $4.2 \%\left(P=1.39 \times 10^{-30}\right)$ of the variance in neuroticism. In all three samples, the PGS reached a maximum prediction accuracy for an infinitesimal prior, indicating a highly polygenic signal.

We refer to Supplementary Table 14 for the full results for all three hold-out samples and for all thresholds.

\section{GWA meta-analysis in METAL}

The direction of the lead SNPs across the 3 cohorts was largely coherent. Of the 170 lead SNPs, 43 (38) had a positive (negative) direction of effect in all three samples. Another 38 (40) had positive (negative) effects in both the UKB and the 23andMe sample, but were not observed in the smaller GPC1 sample. Furthermore, 10 lead SNPs were observed in all samples, but with varying direction of effects (for 6 out of these 10, the direction in the GPC1 sample differed from the other two samples). One of the 170 lead SNPs was only observed in the UKB sample. Overall, we conclude that most lead SNPs had concordant direction of effects across the three cohorts, strengthening our confidence in the results (see Supplementary Tables 2 and 3 for all results)

\section{Genomic risk loci and functional annotations}

The meta-analysis resulted in 170 independent lead SNPs $\left(r^{2}<0.1\right)$ in 138 independent genomic risk loci (loci $<250 \mathrm{~KB}$ apart were merged into 1) (Supplementary Table 3). The borders of the genomic risk loci were defined by taking all independent GWS SNPs at $r^{2}<0.6$ and then determining all SNPs that were in LD with one of the independent GWS SNPs, as detailed in Online Methods (we provide an overview of lead SNPs reported earlier for neuroticism in Supplementary Table 4). Annotated SNPs are all SNPs that are located in a risk locus and that are in LD with one of the independent GWS SNPs. After inspection of the regional plots (LocusZoom) of all genomic loci $(n=138)$ initially identified in SNP-based analysis, we decided that 2 loci had low confidence, as these appeared to be driven by a single, or only a few SNPs. 
To further examine whether these loci are plausible, we conducted a number of checks. First, we compared allele frequencies of the lead SNPs in these loci in UKB data to the allele frequency reported by 1000 Genomes and TOPMED, as a large difference could indicate genotyping errors (Supplementary Table 5). Secondly, the SNP association $P$ values for the lead SNPs were compared across the three individual cohorts to see whether the signal was exclusively driven by a single cohort. Finally, differential LD across datasets might be another indicator of genotyping errors. We gathered all SNPs in LD $\left(r^{2}>0.6\right)$ with the lead SNPs in the 1000 Genomes data, and compared the LD in the 1000 Genomes data with LD (for the same combinations of SNPs) in the UKB data (Supplementary Table 6). For the 2 loci, there was only one lead SNP between 1 to 5 other GWS SNPs. The two lead SNP $P$ values were $8.05 \times 10^{-9}$ and $2.87 \times 10^{-8}$ and were mostly driven by the UKB sample. However, this was also the largest sample $\left(\mathrm{N}_{\mathrm{UKB}}=372,903 ; \mathrm{N}_{23 \text { andMe }}=59,206\right.$; $\mathrm{N}_{\mathrm{GPC} 1}=17,375$ ). For both lead SNPs, the $P$ value from the meta-analyses was slightly lower than the $P$ value for that SNP in UKB, indicating that there was also signal in the 23 andme and GPC1 samples. MAF of the lead SNPs were comparable to the MAF in $1000 \mathrm{G}$ and TOPMED (Supplementary Table 5). The lead SNPs did not have many LD proxies in our meta-analyses (where UKB was used as a reference to calculate LD), yet these same SNPs also do not have a lot of LD proxies in the independent reference sample of $1000 \mathrm{G}$. There were thus no clear indications of genotyping errors of these SNPs, yet we felt that the current evidence for these 2 loci is based on very few SNPs and therefore we have less confidence in these loci, and count the total number of genomic loci for neuroticism as $138-2=136$. We do provide information on these loci (risk loci 105 and 114) in Supplementary Tables 5-6.

All chromosomes except chromosome 21 (chromosome $\mathrm{X}$ was not included in the analysis) included one or more risk loci. The strongest signal was on chromosome 17 (risk locus 126), overlapping an inversion that was previously associated with neuroticism. Despite the presence of this inversion and the resulting long-range LD in this locus, there were 25 independent $\left(r^{2}<0.6\right)$ significant SNPs and 3 lead SNPs $\left(r^{2}<0.1\right.$; rs77804065; rs199447; rs17698176). rs77804065 is in the inversion, but rs199447 and rs 17698176 are more distal and are both in intronic regions of NSF. The closest exonic SNP rs199533 has an $r^{2}$ of 0.991 with lead SNP rs19944 and is also in NSF, and has a CADD score of 13.16 suggesting it is likely deleterious. This SNP is a synonymous coding SNP yet acts as an eQTL for multiple genes across several tissues.

The second most associated locus (locus 130, Supplementary Table 3) on chromosome 18 includes 2 lead SNPs. The top lead SNP is rs11082011 $\left(2.54 \times 10^{-23}\right)$, which is in an intronic region of the CELF4 gene. The second lead SNP in this locus is rs 17570807 and is intergenic, 5,458 bp from MIR4318 (Supplementary Table 12). There are no exonic variants in this locus, but there are several intronic SNPs in the CELF4 gene with high CADD scores. CELF4 is the only mapped protein-coding gene in this locus. Full details of all neuroticism risk loci and functional implications are listed in Supplementary Table 12.

Partitioned heritability showed enrichment of several functional genomic categories (Supplementary Table 13), with the strongest enrichment in conserved regions (Enrichment of 13.791, $\left.\mathrm{SE}=1.420, h^{2}=.359, \mathrm{P}=5.14 \times 10^{-16}\right)$. FUMA ${ }^{216}$ annotated all 17,794 SNPs that were in LD 
$\left(r^{2}>=0.6\right)$ with one of the independent significant SNPs and allowed further inspection of specific coding variants. The most likely genetic variants to have a substantial outcome on a phenotype are those that have a direct consequence on a protein, specifically variants located in coding exons that have a nonsynonymous change resulting in a change to the protein's amino acid sequence (ExNS SNPs). Of the annotated 17,794 SNPs in GWS loci, we identified 70 ExNS located in 47 unique genes (Table 1, Supplementary Table 12). Eleven genes included more than 1 ExNS: SPPL2C (8 ExNS), MAPT (6 ExNS), FAM120AOS (3 ExNS), ANKK1 (3 ExNS), GNL3, ITIH1, RPS6KL1, ZNF646, CHR1, KANSL1 and ASXL3 (all 2 ExNS). All ExNS variants were in high $\operatorname{LD}\left(r^{2}>=0.6\right)$ with one of the GWS lead SNPs, and 46 were GWS themselves. 25 ExNS had CADD scores above $12.37^{197}$ (Table 3.1), and 19 ExNS were likely to affect binding sites.

\section{Mapped genes by FUMA based on GWS SNPs in risk loci and gene-based analysis}

We used the three gene-mapping strategies implemented in FUMA to select genes of interest based on GWS SNPs in the 136 risk loci (Supplementary Table 15). For positional mapping, we mapped SNPs in the risk loci and in LD with the independent GWS SNPs to genes using a window of 10 $\mathrm{Kb}$, resulting in 283 mapped genes. eQTL mapping resulted in 369 genes, of which 159 were outside of risk loci, and chromatin interaction mapping resulted in 119 genes, of which 6 were outside of the risk loci. MAGMA gene-based test resulted in 336 significantly associated genes, and 203 were also mapped by at least one the FUMA gene mapping strategies. Of the resulting set of 599 unique genes, 120 had a $\mathrm{pLI}>0.90$, indicating that these were extremely intolerant to loss of function mutations (Supplementary Table 15). Of the 50 genes that were implicated by all four strategies, DRD2 had the lowest gene-based P value $\left(3.43 \times 10^{-24}\right)$.

\section{Gene-set analyses}

We conducted gene-set analysis on a total of 7,323 gene-sets: 7,246 sets derived from the MsigDB, 53 tissue specific gene-sets and 24 cell specific gene-sets. The threshold for significance of genesets was thus set at $0.05 / 7,323=6.83 \times 10^{-6}$ and competitive testing was used throughout (Online Methods).

Gene-set analysis using gene-sets from MsigDB version 6.0 in MAGMA resulted in 7 associated gene-sets (Supplementary Table 17-18). We then used conditional gene-set analyses to determine which MsigDB gene-sets represent independent associations (Online Methods), which reduced this to three gene-sets that showed independent interaction and were driving the association with the other gene-sets (Supplementary Fig. 16, Supplementary Table 19). Of the 1,402 genes in the neurogenesis gene-set, 51 were implicated by positional, eQTL, or chromatin interaction mapping and 49 genes had a GWS gene-based $P$ value. Of the 12 genes in the behavioral response to cocaine set, 3 genes were implicated by positional, eQTL, or chromatin interaction mapping and also had a GWS gene-based $P$ value. Of the 219 genes in the axon part set, 11 genes were implicated by positional, eQTL, or chromatin interaction mapping and 13 genes had a GWS 
gene-based $P$ value (Supplementary Table 16). When conditioning these gene-set association of neuroticism on the subclusters depressed affect and worry, we observed that the association with 'axon-part' only remained after conditioning for depressed affect $\left(P=2.42 \times 10^{-6}\right)$, but not on worry ( $P=0.0013)$. This suggests that items from the worry subcluster largely explain the involvement of this gene-set in neuroticism, whereas this gene-set is not likely to play a role in items related to depressed affect.

We additionally conducted gene-set analysis on 53 sets of genes that were tissue-specific, based on GTEx gene-expression values (Online Methods). We found Bonferroni corrected significant association for 6 tissue specific gene-sets and all of these were brain tissues, with the strongest association in brain frontal cortex (Supplementary Tables 17-18).

Cell-type specific gene-set analysis, using brain cell type expression data drawn from scRNAseq data from mouse brain ${ }^{221}$, showed significant association with 5 cell types: medium spiny neurons, serotonergic neurons, interneurons and dopaminergic neuroblasts, and embryonic midbrain nucleus neurons (Supplementary Table 21).

We note that the fact that cell type expression data was derived not from humans but from mice, inevitably raises the question whether the results are applicable to the human species. Further research on the comparability of this method is required, yet studies thus far indicate that the results of this analysis are likely to generalize to human cells. First, only genes with a one-to-one mapping between mice and human were included in the analyses. Secondly, previous studies indicate that gene expression clusters not by species but by tissue or cell type ${ }^{221}$. Finally, for schizophrenia results have been shown to replicated in human single-cell data ${ }^{221}$. Importantly, the use of mouse cell-type expression data rather than human data has specific advantages. For instance, postmortem gene expression data in human cells is often of lower quality than mouse expression data, e.g., because time between death and measurement is much shorter in mice. In addition, only single nuclei sequencing is possible in humans, whereas isolation of whole cells (excluding distal neurites) is possible in mice. For an extensive discussion, we refer to Skene et al. ${ }^{221}$.

While the translation from human personality and mood disorders to animal models is challenging, the relevance of animal, and specifically mouse, models for the genetic and neuroscientific study of human psychiatric traits like neuroticism, and to neuroticism-related traits, such as anxiety and depression, is generally acknowledged ${ }^{237-241}$.

\section{GWAS meta-analysis results of secondary phenotypes, not previously published}

\section{Depression}

Meta-analysis of the depression GWAS in UKB, 23andMe and PGC was carried out in METAL ${ }^{179}$. As the UKB GWAS concerned a continuous operationalization of the depression phenotype, while 23andMe and PGC used case-control phenotypes, the odds ratio from the 23andMe and PGC summary statistics were converted to log odds, reflecting the direction of the effect. The metaanalysis was then performed on the $P$ value of each SNP using a sample size-weighted fixed-effects 
analysis. The genetic signal correlated moderately between the three samples $\left(r_{g}\right.$ range: $0.61-.80$; Supplementary Table 22).

The quantile-quantile (Q-Q) plot of the genome-wide meta-analysis on 688,809 subjects showed high inflation $(\lambda=1.44)$ and mean $\chi^{2}$ statistic (1.55) (Supplementary Fig. 8; Supplementary Table 22, also for the 3 samples separately). The LD Score regression (LDSC) ${ }^{146}$ intercept (1.007; $\mathrm{SE}=0.010)$ was consistent with inflation due to true polygenicity and large sample size $^{196}$. The LDSC SNP-based heritability $\left(h^{2}{ }_{S N P}\right)$ of depression was 0.040 (SE=0.002).

Our depression phenotype is a continuous one, based on two Likert scale items. A true casecontrol phenotype is not present in the UKB data base, but other researchers have proposed casecontrol operationalizations for depression by combining answers to multiple items (e.g., Howard et al. ${ }^{229}$, Wray et al. ${ }^{230}$ ). To test whether our GWAS meta-analysis results are very different from results that we would have obtained with a case-control phenotype, we ran an additional metaanalysis but now with the 'probable depression' case-control phenotype as proposed by Howard et al. ${ }^{229}$. The genetic correlation between our meta-analysis results, and the meta-analysis results based on that case-control phenotype was very high $\left(r_{g}=0.930, \mathrm{SE}=0.010\right)$. This motivated us to continue with our continuous measure of depression, as this score was available in a considerably larger sample (i.e., 362,696 versus 174,519, respectively).

The depression GWAS meta-analysis identified 1,960 genome-wide significant (GWS) SNPs $\left(P<5 \times 10^{-8}\right)$ (Supplementary Table 24). FUMA extracted 49 independent lead SNPs, which were mapped to 45 independent genomic loci harboring 173 genes (Supplementary Table 28). MAGMA $^{242}$ identified 119 GWS genes (Supplementary Table 16), of which 61 overlapped with genes implicated by SNP-based analyses, resulting in a total of 234 genes associated with depression.

\section{Neuroticism subcluster: Depressed affect}

The quantile-quantile (Q-Q) plot of the genome-wide analysis on 357,957 subjects and 10,847,151 SNPs showed high inflation $(\lambda=1.51)$ and mean $\chi^{2}$ statistic (1.67) (Supplementary Table 23). The $\operatorname{LDSC}^{116,146}$ intercept (1.038; $\left.\mathrm{SE}=0.010\right)$ was consistent with inflation due to true polygenicity and large sample size. The LDSC SNP-based heritability $\left(h^{2}{ }_{S N P}\right)$ of depressed affect was 0.089 $(\mathrm{SE}=0.003)$.

The depressed affect GWAS analysis identified 5,240 GWS SNPs $\left(P<5 \times 10^{-8}\right)($ Supplementary Table 25). FUMA extracted 75 independent lead SNPs (see Supplementary information for definition of lead SNPs), which were mapped to 66 independent genomic loci harboring 246 genes (Supplementary Table 29). MAGMA ${ }^{242}$ identified an additional 94 unique genes (Supplementary Tables 16), resulting in a total of 340 genes associated to depressed affect.

\section{Neuroticism subcluster: Worry}

The quantile-quantile (Q-Q) plot of the genome-wide analysis on 348,219 subjects and 10,847,151 SNPs showed high inflation $(\lambda=1.49)$ and mean $\chi^{2}$ statistic (1.65) (Supplementary Table 23). The 
$\operatorname{LDSC}^{116,146}$ intercept (1.021; $\left.\mathrm{SE}=0.010\right)$ was consistent with inflation due to true polygenicity and large sample size. The LDSC SNP-based heritability $\left(h^{2}{ }_{S N P}\right)$ of worry was 0.090 (SE=0.003).

The worry GWAS analysis identified 6,382 GWS SNPs $\left(P<5 \times 10^{-8}\right)$ (Supplementary Table 26). FUMA extracted 73 independent lead SNPs (see Supplementary information for definition of lead SNPs), which were mapped to 68 independent genomic loci harboring 332 genes (Supplementary Table 30). MAGMA ${ }^{242}$ identified an additional 115 unique genes (Supplementary Table 16), resulting in a total of 447 genes associated to worry.

\section{SNP-based heritability of the neuroticism item clusters}

The $h^{2}{ }_{S N P}$ estimates of the subclusters (both .09; see Supplementary Table 23) are only slightly lower than the $h_{S N P}^{2}$ estimate of the total sum-score (.10). Generally, if one sums items that are genetically correlated, then the signal-to-noise ratio (in this case: the ratio of genetic variance to the total variance, i.e., the heritability) will increase with the number of items, i.e., we expect higher $h^{2}{ }_{S N P}$ if we sum more genetically correlated items. Therefore, the finding that the $h^{2}{ }_{S N P}$ of the 4 items is only slightly lower as the $h^{2}$ SNP of the 12 items confirms their genetic homogeneity.

It is crucial to note that the higher $h^{2} S N P$ of the sum of the 12 items does not necessarily imply that gene-finding studies using this sum score as dependent variable have enhanced power to identify underlying genetic variants, as higher $h^{2}$ SN does not imply larger single SNP effect sizes.

\section{Functional annotation results for the two neuroticism item clusters}

Of the 9,888 $(10,161)$ SNPs in high LD with one of the independent significant SNPs for the depressed affect (worry) cluster, most were intronic (depressed affect: 5,387 =54.9\%; worry: 4,707 $=46.3 \%$ ) or intergenic (depressed affect: $2,249=22.9 \%$; worry: 3,351 $=33.0 \%$; Supplementary

Table 31-33; Supplementary Fig. 15). For depressed affect 1.1\% $(n=104)$ of the SNP was exonic, with 49 SNPs being non-synonymous (ExNS) and 1 splicing variant (Supplementary Table 34). For worry $1.33 \%(\mathrm{n}=135)$ of the SNPs were exonic, of which 66 were non-synonymous, and 1 was a splicing variant (Supplementary Table 34). Of all ExNS SNPs, 22 overlapped between the clusters, of which 19 are located in a well-known inversion on chromosome $17^{141}$. Although some ExNS SNPs were also identified in the meta-analysis of the neuroticism sum-score (27 and 39 for depressed affect and worry, respectively), we found 12 ExNS SNPs that were specifically associated to the depressed affect cluster, and 15 ExNS SNPs that are uniquely associated to the worry cluster. This suggests that among those SNPs that are highly likely to have functional consequences, the clusters are 1) distinct and 2) adding information to the results of neuroticism sum-score analysis.

As an example, we highlight here for each cluster one cluster-specific ExNS SNP, not identified for the neuroticism sum-score, that may be a viable candidate for functional follow-up studies. For depressed affect, the ExNS SNP with the highest CADD score (35) was rs45510500, located in exon 40 of KIAA1109. rs 45510500 is a missense mutation that leads to an amino acid change of 
Arginine to Tryptophan, of which the $\mathrm{T}$ allele is associated with a lower score on the depressed affect cluster. For the worry cluster, one of the SNPs with the highest CADD score (26.9) is rs 13072536, in exon 3 of ITIH1, and is a missense mutation resulting in a Isoleucine to Asparagine change. The $\mathrm{T}$ allele is associated to a lower score on the worry cluster. rs 13072536 has a regulome database score of $1 \mathrm{f}$, meaning that it is likely to affect binding and to affect expression of a gene target.

\section{Genetic correlation analysis}

Neuroticism has been phenotypically linked to social, and both mental and physical health-related outcomes $^{131,243-245}$, and genetically to mental and physical health-related outcomes. We used LDSC to calculate genetic correlations between neuroticism and 35 traits from large GWAS studies for which summary statistics were publicly available (an overview of the studies used in this analysis is given in Supplementary Table 36). To be able to compare the genetic correlational pattern of neuroticism to that of depression, depressed affect and worry, we also calculated the genetic correlations of these three traits to the 35 external traits. These are all shown in Figure $\mathbf{4}$ of the main text and Supplementary Table 37.

The $r_{\mathrm{g}}$ 's between our 4 traits are shown in Supplementary Table 23. Note that the $r_{\mathrm{g}}$ between depression and depressed affect was .895 , while the $r_{\mathrm{g}}$ 's of depression and depressed affect with worry were only $r_{\mathrm{g}}=.582$ and $r_{\mathrm{g}}=.629$, respectively. The $r_{\mathrm{g}}$ 's of depression and depressed affect with other traits strongly mirrored each other (correlation between their $r_{\mathrm{g}}$ 's is $\mathrm{r}=.98 ; \mathrm{r}=.70$ was between $r$ 's of depression and worry), validating the depressed affect cluster.

We observed 11 Bonferroni significant genetic correlations for neuroticism $(\alpha=0.05 /(4 \times 35)$; $P<3.6 \times 10^{-4}$ ) (Fig. 3.4; Supplementary Table 37). We observed 17 Bonferroni significant genetic correlations for depression, 15 for depressed affect, and 13 for worry.

The genetic distinctness of the two clusters depressed affect and worry is apparent from the differences in their genetic relations to other traits. For instance, worry is significantly correlated to anorexia, schizophrenia and bipolar disorder, and to childhood body mass index (BMI) and childhood obesity, while depressed affect is not. Depressed affect, in turn, is significantly related to ADHD, waist-to-hip ratio, ever smoking, smoking cessation, longevity, age of having first child and number of children, while worry is not. For waist circumference, BMI, and hip circumference their genetic correlations are even in opposite directions.

Corroborating previous reports ${ }^{131,141,191}$, neuroticism was genetically correlated to various psychiatric traits ( $r_{g}$ range: .20-.82) and to subjective well-being $\left(r_{g}=-.68\right)$. Interestingly, the $r_{\mathrm{g}}$ 's of neuroticism with anorexia, schizophrenia, and bipolar disorder seem to originate mainly from the worry cluster, suggesting that the genetic link between neuroticism and these disorders may be founded on shared worry/tense/irritable features. In contrast, the $r_{\mathrm{g}}$ of neuroticism with ADHD seems to originate mainly from the depressed affect cluster, which is in concordance with a large body of literature on the relation between ADHD and depression. Notably, neuroticism did not significantly correlate to e.g. BMI- and smoking-related phenotypes, while the two clusters did, 
albeit in opposite directions. Together, these $r_{\mathrm{g}}$-based findings corroborate the complexity of the neuroticism phenotype, and illustrates how insight can be gained by distinguishing genetically homogeneous sub-dimensions.

\section{Mendelian randomization}

To investigate whether the observed genetic correlations between the four studied traits and the 35 external traits for which large-scale summary statistics were available (Supplementary Table 39) could be explained by directional associations, we performed Mendelian randomization (MR) based on GWAS summary statistics, using generalized summary-data-based Mendelian randomization $\left(\mathrm{GSMR}^{205}\right)$. The MR analysis of neuroticism and depression showed strong bidirectional associations (neuroticism on depression: $b_{x y}=0.488, P<1 \times 10^{-300}$; depression on neuroticism: $b_{x y}=0.934, P=1.87 \times 10^{-252}$ ), implying that neurotic traits increase the liability to depression, while depression in turn results in a more neurotic personality. Neuroticism also showed significant causal relations with three other psychiatric disorders, namely anxiety disorder (strong unidirectional positive effect going from neuroticism to anxiety), ADHD and schizophrenia (both positive and bidirectional). These directional associations between neurotic traits and psychiatric disorders imply that neurotic personality characteristics are both a causal factor and a consequence of psychiatric illnesses. The former suggests that preventive treatment of neurotic tendencies (e.g., reduction of negative beliefs through cognitive behavioral therapy) may decrease the risk of developing psychiatric problems. Indeed, prior studies indeed have shown that individuals scoring high on neuroticism tend to have worse health outcomes compared to individuals low on neuroticism ${ }^{246}$.

Analyzing directions of causation for the 21 traits that had significant genetic correlations to at least one of the four phenotypes in LD Score regression (LDSC) analyses (Supplementary Table 37), we observed mostly bidirectional negative causal effects for educational attainment (all phenotypes except worry, $b_{x y}$ range: $-0.326--0.165, P<2.98 \times 10^{-5}$ ) and IQ (neuroticism and depressed affect, $b_{x y}$ range: $-0.324--0.086, P<1.92 \times 10^{-5}$; Supplementary Table 39). Positive bidirectional causal associations were observed between schizophrenia and all four phenotypes $\left(b_{x y}\right.$ range: $0.608-1.654, P<1.25 \times 10^{-9}$ ), indicating that increased liability to neurotic traits and depression causes increased liability to schizophrenia, and vice versa. In addition, significant positive unidirectional associations were observed in the reverse analyses between BMI and both depression and depressed affect (depression, $b_{x y}=0.061, P=4.96 \times 10^{-12}$, depressed affect, $b_{x y}=0.049$, $\left.P=5.35 \times 10^{-6}\right)$, suggesting that obesity enhances the probability to develop depression.

Interestingly, the depressed affect and worry clusters showed distinct patterns of causality which partly resembled the observed differences in genetic correlations by LDSC. For instance, bidirectional negative (rather than positive) associations were observed between worry and BMI

(forward: $b_{x y}=-0.236, P=2.10 \times 10^{-10}$; reverse: $b_{x y}=-0.123, P=4.87 \times 10^{-15}$ ), while BMI only affected depressed affect positively (as noted above) and not the other way around $\left(b_{x y}=0.033, P=0.39\right.$ ). 
These results suggest that a lower liability to worry may decrease the probability to become obese, whereas a lower liability to the other sub dimension of neuroticism, depressed affect, does not cause decreased liability to obesity. We note that several GSMR analyses showed significant causality between traits that did not show a significant genetic correlation by LDSC, which may be explained by the use of significant variants as instrumental variable in GSMR analysis instead of using all SNPs in LDSC. An overview of the results can be found in Supplementary Table 39.

\section{Drug targets}

We explored whether genes implicated by the GWAS and GWGAS results may provide potential targets for pharmacotherapy treatment by searching the drug-gene interaction database $\left(\right.$ DGIdb $\left.^{206,207}\right)$, a large online collection of drug-interaction databases (Online Methods). First, we selected genes associated with any of the four traits, and searched for known interactions with existing FDA approved therapeutic compounds. This resulted in 67 unique genes with reported drug interactions with 491 unique pharmaceuticals (average 7-8 compounds per gene, Supplementary Table 40). The $H R H 1$ gene that was implicated in depression showed the most reported interactions (130 interactions), of which most were antagonists of the gene-product $(\mathrm{n}=124,95.4 \%)$. HRH1 codes for the histamine receptor H1, an important neurotransmitter in the central nervous system ${ }^{247}$. Among all interactions with the $H R H 1$ gene, the highest DGIdb score (i.e. the number of distinct sources mentioning the interaction) was found for Loratadine (score=16) which is a frequently used antihistamine medication for the treatment of common allergies. In addition, a large number of known interactions $(\mathrm{n}=104)$ was observed for the DRD2 gene, coding the dopamine receptor 2 (implicated in all four traits), of which most interactions were antagonistic $(\mathrm{n}=65,62.5 \%)$. These results are in line with extensive previous literature on the role of dopamine receptor in psychiatric disorders ${ }^{248}$. When comparing results between the four phenotypes, we observed a number of potentially druggable genes specific for neuroticism and depression/depressed affect, but not for worry, including CACNA1E (5 interactions) and NOS1 (1 interaction). However, we note that this search mainly results in finding interactions that have already been extensively studied in psychiatric disorders, while many more yet untargeted genes may exist. For this reason, in order to find novel drug targets, we performed a search in 10 databases in DGIdb of potentially druggable genes based on the type of target coded by the gene. We found a large number of potential interactions based on the gene-target (Supplementary Fig. 17, Supplementary Table 41), covering 168 unique genes, of which the large majority ( $n=126,75 \%)$ had not previously been reported to interact with approved medication.

These results show that GWAS is able to identify a large set of potentially clinically relevant genes. Indeed, genes that have been identified by GWAS have been shown to have a higher success rate to make it to the approved drug market ${ }^{249}$. Several limitations, however, still exist that need to be overcome to translate these findings into direct clinical use. Given the wealth of genes that are currently identified, prioritizing genes for therapeutic intervention becomes challenging. Performing trial-and-error clinical investigations of potential gene-targets based on these results is 
expensive and time-consuming, and thus not a feasible solution. We argue that one possible way to select which gene targeting may be most effective, is by identifying 'core genes' that play a central role in gene networks ${ }^{250}$, which requires improved insight into underlying connections between genes (i.e. gene co-expression or protein-interactions). Furthermore, as has been pointed out previously ${ }^{249}$, it is unclear which genes mapped by GWAS actually play a causal role, and the inclusive approach of including all mapping methods may lead to an overestimation of the actual number of targetable genes. In addition, given small effect sizes of these genes and the highly polygenic architecture of psychiatric disorders, we argue that targeting single genes may only provide limited results, and we may require multi-targeted therapy in order to yield effective therapies.

\section{Comparison with Luciano et al. (2017)}

Here we provide a brief discussion of how our results compare to those presented in a study conducted in the same period and which was published while our manuscript was under consideration $^{251}$. We present a brief overview of which loci overlap with those reported in Luciano et al. and which can still be considered novel. To this end, we checked for overlap between the loci identified in the current study, and the 90 non-overlapping loci reported for the 116 independent GWS SNPs reported by Luciano et al. (we refer to their Supplementary Materials for details). Of the 124 loci initially reported in the current study as being novel, 51 overlap with a locus as reported by Luciano et al., while 73 loci identified in our study are not reported in their study, i.e. are novel (Supplementary Table 42). Reversely, of the 90 loci reported in Luciano et al., 24 were not GWS in our current, larger sample.

The surplus of novel loci in our study compared to that of Luciano et al., is likely due to a difference in sample size $(\mathrm{N}=329,821$ in the discovery sample of Luciano et al. versus $\mathrm{N}=449,484$ in the current study) and ensuing increased statistical power of the current study. In addition, subtle differences in selection of SNPs, covariates and/or phenotype operationalization may have prevented some SNPs to exceed the genome-wide significance threshold in Luciano et al., whereas they did in our study (obviously, the reverse is likely to be true as well).

\section{Acknowledgements and notes}

\section{Acknowledgements}

This work was funded by The Netherlands Organization for Scientific Research (NWO Brain \& Cognition 433-09-228, NWO MagW VIDI 452-12-014, NWO VICI 435-14-005 and 453-07-001, 645-000-003). P.R.J. was funded by the Sophia Foundation for Scientific Research (SSWO, grant nr: S14-27). J.H.L. was funded by the Swedish Research Council (Vetenskapsrådet, award 20143863), StratNeuro, the Wellcome Trust (108726/Z/15/Z), and the Swedish Brain Foundation 
(Hjärnfonden). N.S. was supported by the Wellcome Trust (108726/Z/15/Z). JB was funded by the Swiss National Science Foundation. Work of H.T. was supported by a NWO-VICI grant (NWOZonMW: 016.VICI.170.200). Analyses were carried out on the Genetic Cluster Computer, which is financed by the Netherlands Scientific Organization (NWO: 480-05-003), by the VU University, Amsterdam, the Netherlands, and by the Dutch Brain Foundation, and is hosted by the Dutch National Computing and Networking Services SurfSARA. This research has been conducted using the UK Biobank Resource (application number 16406). We would like to thank the participants, including the 23 andMe customers who consented to participate in research, and researchers who collected and contributed to the data.

\section{Author contributions}

S.vd.S and D.P. conceived the study. M.N. and P.R.J. performed the analyses. S. St. performed the quality control on the UK Biobank data and wrote a pipeline to facilitate data processing. K.W. constructed the tool for biological annotation and ran the analyses. N.S., A.B.M.M., S.L. and J.H.L. provided single-cell RNA-seq data for mouse brain cell types. J.B. and P.F.S. performed the singlecell gene-expression analysis. M.N., P.R.J., S.vd.S and D.P. wrote the paper. All authors discussed the results and commented on the paper.

\section{3andMe Research Team contributors}

Michelle Agee, Babak Alipanahi, Adam Auton, Robert K. Bell, Katarzyna Bryc, Sarah L. Elson, Pierre Fontanillas, Nicholas A. Furlotte, David A. Hinds, Bethann S. Hromatka, Karen E. Huber, Aaron Kleinman, Nadia K. Litterman, Matthew H. McIntyre, Joanna L. Mountain, Elizabeth S. Noblin, Carrie A.M. Northover, Steven J. Pitts, J. Fah Sathirapongsasuti, Olga V. Sazonova, Janie F. Shelton, Suyash Shringarpure, Chao Tian, Joyce Y. Tung, Vladimir Vacic, and Catherine H. Wilson.

\section{Data availability}

Our policy is to make genome-wide summary statistics (sumstats) publicly available. Sumstats from our neuroticism meta-analysis, our depression meta-analysis, and the GWA analyses for depressed affect and worry are available for download at the website of the department of Complex Trait Genetics, CNCR (see URLs).

Note that our freely available meta-analytic sumstats concern results excluding the $23 \mathrm{andMe}$ sample. This is a non-negotiable clause in the 23 andMe data transfer agreement, intended to protect the privacy of the 23 andMe research participants. To fully recreate our meta-analytic results for neuroticism: (a) obtain Lo et al. (2016) sumstats from 23andMe (see below); (b) conduct a metaanalysis of our sumstats with the Lo et al. sumstats. To fully recreate our meta-analytic results for 
depression: (a) obtain Hyde et al. (2016) sumstats from 23andMe (see below); (b) conduct a metaanalysis of our sumstats with the Hyde et al. sumstats.

23andMe participant data are shared according to community standards that have been developed to protect against breaches of privacy. Currently, these standards allow for the sharing of summary statistics for at most 10,000 SNPs. The full set of summary statistics can be made available to qualified investigators who enter into an agreement with $23 \mathrm{andMe}$ that protects participant confidentiality. Interested investigators should contact David Hinds (dhinds@23andme.com) for more information.

\section{URLs}

UK Biobank, http://www.ukbiobank.ac.uk/

MAGMA, http://ctg.cncr.nl/software/magma

MSigDB, http://software.broadinstitute.org/gsea/msigdb/collections.jsp

METAL, http://genome.sph.umich.edu/wiki/METAL_Program

LDSC, https://github.com/bulik/ldsc

FUMA, http://fuma.ctglab.nl/

GSMR, http://cnsgenomics.com/software/gsmr/

DGIdb, http://dgidb.org/

Ethical and Independent Review Services, http://www.eandireview.com/

Genetics of Personality Consortium, http://www.tweelingenregister.org/GPC/

Psychiatric Genomics Consortium, http://www.med.unc.edu/pgc/results-and-downloads

GTEx Portal, https://www.gtexportal.org/home/

GWAS summary statistics https://ctg.cncr.nl/software/summary_statistics. 



\section{$4 \quad$ Examining the genetic validity of internalizing disorders through item-level analyses}

Mats Nagel ${ }^{1,2 \S^{*}}$, Jeanne E Savage ${ }^{1 \S}$, Kyoko Watanabe ${ }^{1}$, Danielle Posthuma ${ }^{1,2}$, Sophie van der Sluis ${ }^{1,2}$

1. Department of Complex Trait Genetics, Center for Neurogenomics and Cognitive Research, Amsterdam Neuroscience, Vrije Universiteit, Amsterdam, Amsterdam, The Netherlands

2. Department of Child Psychiatry, Section Complex Trait Genetics, Amsterdam Neuroscience, Vrije Universiteit Medical Center, Amsterdam UMC, Amsterdam, the Netherlands

$\S \quad$ These authors jointly supervised this work

Manuscript in preparation. 


\section{Abstract}

The genetics of internalizing psychiatric traits (depression, anxiety) are extremely challenging to unravel due to pervasive pleiotropy, correlated genetic liabilities, and symptom overlap between disorders. By using genetic information on 34 self-reported internalizing symptoms from the UK Biobank we aim to disentangle the genetics of these traits and study the genetic validity of traditionally defined internalizing constructs.

Exploratory factor analysis on the genetic correlations between these 34 items revealed 4 correlated, genetically homogeneous factors. Conducting GWAS on these 4 genetically derived clusters as well as on the 3 traditional scales that the 34 items pertained to (depression, anxiety, neuroticism), showed high genetic correlations between all clusters, indicating that genetic influences were broadly shared across all internalizing symptoms. However, the four distinguishable genetic factors were associated with partially distinct genomic loci and showed some divergent genetic correlations with psychiatric disorders and health-relevant outcomes, although the pattern was broadly similar between factors.

Our results support the emerging evidence from twin and molecular studies that traditional diagnostic definitions of psychiatric constructs do not always accurately represent their underlying genetic etiology. In parallel to ongoing efforts to increase sample sizes, our findings inspire future genetic studies of psychiatric disorders to employ fine-phenotyping to address the genetic validity of generally used diagnostic constructs. 


\subsection{Introduction}

Increasing availability of large genetic data sets has facilitated gene identification for many complex traits ${ }^{41}$. Although genome-wide association studies (GWAS) have proven fruitful for some traits, such as schizophrenia (SCZ) ${ }^{164}$, many psychiatric disorders with modest to moderate heritability, like major depression (MDD), anxiety disorders (ADs), and substance use disorders (SUDs), have remained particularly resistant to gene identification success ${ }^{67,72,141,252}$.

The current approach of studying the genetics of these traits reflects the assumption that they are genetically coherent (i.e., genetic variants affect all symptoms of a disorder in the same way) and genetically distinct from one another. However, it may be that the binary diagnostic categories typically used in psychiatric genetic research may obscure the heterogeneous nature of the disorders, which may actually reflect underlying genetic heterogeneity. Specifically, the same diagnosis can be applied to individuals meeting a threshold number of symptoms, even if their constellations of symptoms may be largely, or even entirely, different ${ }^{253}$. If the genetic etiology differs between symptoms/symptom clusters - for which there is evidence in MDD ${ }^{122,123}$, SUDs ${ }^{254}$ ${ }^{256}$, and $\mathrm{SCZ}^{257}$ - statistical power for gene discovery will be reduced when aggregates of symptoms or case-control status variables are used in genetic analyses ${ }^{85}$.

Additionally, comorbidity is widespread within and between classes of psychiatric disorders ${ }^{1,105,258}$, prompting speculation that the diagnostic categories such as employed in diagnostic classification systems like the $\mathrm{DSM}^{92}$, are not as clear-cut as they appear. Twin and family studies have shown that internalizing disorders (mood/anxiety-related) share genetic factors with other disorders in the internalizing, but also the externalizing (antisocial/impulsivity-related), spectrum ${ }^{136,137}$. Several studies on twin data reported the genetic correlation between depression and anxiety (both of which are internalizing disorders) to not deviate from unity ${ }^{259-261}$. In contrast, the genetic correlations between internalizing vs. externalizing disorders are generally lower, though still substantial (e.g., genetic correlation between depression and conduct disorder: $0.41^{262}$ ). Moreover, several recent studies suggest that genetic influences may even manifest as a broad liability to all forms of psychopathology ${ }^{263-265}$. If genetic effects indeed extend across traditional diagnostic boundaries, then genetic studies on standard diagnostic constructs may lose statistical power to detect them. Similarly, if traditional diagnostic constructs are not distinguishable on a genetic level, or when diagnostic constructs are genetically heterogeneous, then genetic studies on standard diagnostic constructs may not yield insight into the biology underlying psychiatric disorders. The aim of the current study is, therefore, to study the genetic validity of diagnostic constructs. Specifically, we examine whether traditional diagnostic constructs from the internalizing spectrum are distinguishable on the genetic level.

While the issues of genetic heterogeneity and genetic overlap seem to be at odds, they do in fact stem from the same problem of phenotypic definition; that is, the criteria used to define the phenotypic boundaries of disorders may not map onto their biological definitions. This is perhaps unsurprising due to e.g. the construction of psychiatric disorders in a "top-down" manner from 
expert consensus, rather than a "bottom-up" fashion originating from observable symptoms ${ }^{266}$. However, when one wishes to gain insight into the biological mechanisms that are common to or distinct across psychiatric disorders, working in a bottom-up fashion, i.e., by studying individual symptoms, may provide more insight than studying aggregate phenotypes like diagnoses or sum scores $^{267}$. Advantages of such item-level genetic analyses were recently illustrated for the personality trait neuroticism ${ }^{192}$, showing that neuroticism actually has two genetically distinct pillars. In general, genetic analyses of individual items could provide empirical insight into whether traits that are constructed from these items are genetically homogeneous, and possibly identify genetic heterogeneity within a trait, or genetic dimensions that span across different traits.

In the current study, we investigate whether a symptom-level approach (or item-level approach; we use the words interchangeably here) can supplement our understanding of the genetic etiology of internalizing disorders and related traits. Specifically, we focus on MDD, AD, and neuroticism and aim to see whether symptoms that are traditionally designated to a specific disorder do indeed cluster together on a genetic level. We use factor analysis on the genetic correlations between individual items to empirically identify the genetic factor structure of symptoms, and compare the results with multiple genetic association approaches. We focus on internalizing disorders because the phenotypic definitions are particularly challenging within this spectrum due to overlapping diagnostic criteria, high cross-disorder genetic correlations ${ }^{135-137268}\left(r_{g}\right.$ 's for anxiety disorder vs. depression, anxiety disorder vs. neuroticism and depression vs. neuroticism are $0.85,0.80$ and 0.70 , respectively), and existing knowledge of heterogeneity within neuroticism ${ }^{192}$, the trait most robustly predictive of and genetically correlated with internalizing disorders ${ }^{144,149}$.

The aims of this study are twofold: 1) to identify genetically homogeneous clusters of symptoms; and 2) to compare the genetically derived clusters of symptoms with the theoretically derived clusters (i.e., clinical scale sum scores, corresponding to the traditional classification), through e.g. comparison of their genetic correlations with several psychiatric and health-related phenotypes. I.e., note that we explicitly choose not to focus on cluster-specific gene identification, since the current sample sizes (range $\mathrm{N}=109,990$ - 126,552) are typically insufficient to robustly detect the, usually very small, genetic effects associated to these kinds of complex psychological traits. Genetic correlations between traits suffer less from these power issues since variants that are not genome-wide significant can still contribute to the correlation.

\subsection{Results}

We derived data on 34 internalizing items from the UK Biobank ${ }^{35,269}$ (Supplementary Table 1). Item selection was dictated by sample size, whether items could be considered 'symptoms' resembling diagnostic criteria, and whether items were administered to all UKB participants rather than a (selective) subset (see Supplementary Fig. 1 for a flow chart of the item selection process; 
Methods). The selection of 34 internalizing items consisted of 8 items intended to measure recent depression symptoms (derived from the PHQ- $9^{270}$ ), 7 items intended to measure recent anxiety symptoms (derived from the GAD- $7^{86}$ ), 12 items intended to measure the neuroticism personality trait (derived from the EPQ-RS ${ }^{88}$ ), 2 and 4 items intended to measure prolonged anxiety and depression, respectively, and one additional item intended to measure prolonged irritability.

There was considerable variation in sample size across the 34 items, mostly resulting from the fact that the neuroticism items were administered at baseline, whereas the other items were administered through an online follow-up questionnaire ${ }^{36}\left(\mathrm{~N}_{\min }=106,215 ; \mathrm{N}_{\max }=380,317\right)$. Endorsement rates for each item ranged from $6 \%$ to $55 \%$ (Supplementary Table 2). Phenotypic correlations between the items were generally high, ranging from 0.17 to 0.90 (average $r=.40$; Supplementary Table 3).

\section{Theoretically derived clusters}

In order to obtain scores that are in line with the traditional disorder classifications following DSM criteria, we summed the items that are typically assigned to individual internalizing classifications (i.e., depression, anxiety, and neuroticism, referred to as theoretical scores from here on; Fig. 4.1). An anxiety sum score $(A N X)$ was constructed by summing the scores on the individual items of the GAD-7 ${ }^{86}$; for depression $(D E P)$ the 8 PHQ- $9^{270}$ were summed. The neuroticism sum score (NEU) was computed from the 12 binary items of the EPQ-RS ${ }^{88}$. Finally, an overall internalizing sum score (SUM_INT) was computed by summing all 34 internalizing items, after dichotomizing GAD7/PHQ-9 items (to reflect absence versus any experience of the symptom) to ensure that all items contributed equally to the overall score. Details on the individual items, and how they were combined into clusters, are described in the Methods (see Supplementary Information; Supplementary Tables 5, 6; Supplementary Fig. 3 for a description of an additional phenotypic factor analysis, whose results strongly resembled, but not completely matched the theoretical structure). 


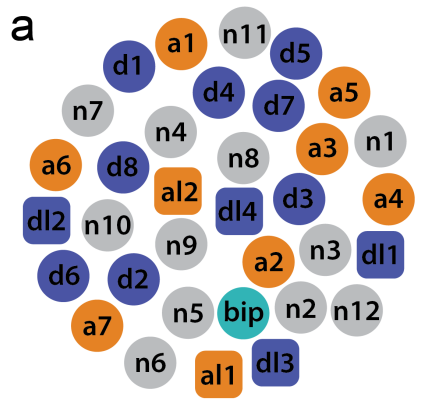

\section{Internalizing items}

a Recent anxiety

items

al Prolonged anxiety

items

d Recent depression

items

dl Prolonged depression items

n Neuroticism items

bip Bipolar checklist irritability

b Theoretically derived clusters

ANX DEP

NEU
a1 a2 a3 a4
d1 d2 d3 d4
$\begin{array}{llllll}n 1 & n 2 & n 3 & n 4 & n 5 & \text { n6 }\end{array}$
a5 a6 a7
d5 d6 d7 d8
n7 n8 n9 n10 n11 n12

\section{Empirically derived genetic level clusters}

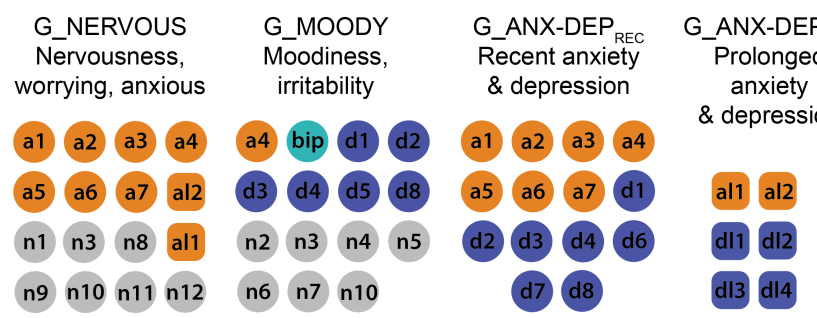

d Empirically derived phenotypic level clusters

\begin{tabular}{|c|c|c|c|c|}
\hline $\begin{array}{l}\text { P_ANX } \\
\text { Recent } \\
\text { anxiety }\end{array}$ & $\begin{array}{l}P_{-}{ }_{\text {DEP }} \\
\text { Recent } \\
\text { depression }\end{array}$ & $\begin{array}{l}\text { P_ANX-DEP } \\
\text { Prolonged anxiety } \\
\text { \& depression }\end{array}$ & $\begin{array}{l}\text { P_MOODY } \\
\text { Moodiness, } \\
\text { irritability }\end{array}$ & $\begin{array}{c}\text { P_NERVOUS } \\
\text { Nervousness, } \\
\text { worrying }\end{array}$ \\
\hline & & al1 al2 & n2 n3 & al2 n1 \\
\hline & & & n4 n5 & n8 $n 9$ \\
\hline & & & n6 n7 & n10 n11 \\
\hline & & & n10 bip & n12 \\
\hline
\end{tabular}

Figure 4.1. Schematic representation of item-to-cluster assignment.

(a) The full set of 34 items in the UK biobank that are indicators of internalizing disorders. All items are color-coded to show the overarching trait/disorder that they collectively measure (e.g., items in red circles were part of the General Anxiety Questionnaire 7-item scale - GAD-7). (b) The conventional, theoretically derived clusters. These clusters represent full-scale scores (e.g. typically, to measure depression, one computes the sum-score of all depression items). Panels (c) and (d) show empirically derived clusters, based on exploratory factor analyses using the genetic (c) and phenotypic (d) inter-item correlations, respectively. Note that in exploratory factor analysis 
(panels c \& d) items may load on more than one factor, i.e., some items are assigned to more than one cluster. See Supplementary Table 9 for an explanation of abbreviations in this figure.

\section{Construction of genetically informed item-clusters}

To extract genetically homogeneous clusters of items, we ran factor analysis on the genetic correlations between the 34 items. Genetic correlations between the items were computed using bivariate linkage disequilibrium (LD) score regression (LDSC ${ }^{116,204}$; Supplementary Table 4; Supplementary Fig. 2; GWAS summary statistics of all 34 individual items are freely available from GWAS ATLAS (URLs).

The genetic factor analysis (i.e., based on the genetic correlations; Supplementary Table 4) did not yield a simple structure solution (Supplementary Tables 7, 8). Particularly the recent anxiety and recent depression items tended to load on more than 1 factor (Supplementary Tables 7, 9; Fig. 4.1). The 4-factor model proved to be most interpretable, as after 4 factors, the factor loading pattern became scattered with most items loading on two or more factors. We chose the 4factor solution 1) because the Eigenvalues $>1$ criterion indicated 4 factors, 2) because we observed a substantial improvement in fit between the 3 and 4 factor solution, but much smaller after that, and 3) because of its interpretability, (relatively) simple structure, and its variance explained of 61\% (Supplementary Tables 7, 8; Supplementary Fig. 4).

The first genetic factor (prefix ' $G$ ') comprised all anxiety items (both recent as well as prolonged) and a subset of the neuroticism items (broadly reflecting the worry component in Nagel et al. ${ }^{192}$ ). Hence, this factor was labeled G_NERVOUS (predominantly symptoms measuring nervousness and anxiousness). The second factor included all three 'irritability' items and 12 other items (from the PHQ-9 and the EPQ-RS) indicating negative affect/moodiness, and was labeled $G_{-} M O O D Y$. The third genetic factor ( $G_{-} A N X-D E P_{R E C}$, covering recent anxious depression) covered all recent anxiety items, and all but one recent depression items. All 6 items that assessed prolonged experience of anxiety or depression across the lifetime loaded strongly on the fourth factor ( $G_{-} A N X-D E P_{P R O}$, prolonged anxious depression). We summed the scores on items assigned to each of these four genetic factors, after dichotomizing items as described above to ensure equal score contributions. Phenotypic correlations among these genetically derived cluster sum scores (genetic scores from here on) ranged from 0.23 (G_NERVOUS with $\left.G_{-} A N X-D E P_{R E C}\right)$ to 0.74 $\left(G_{-} M O O D Y\right.$ with $G \_A N X-D E P_{R E C}$; Table 4.1).

In general, items derived from the same questionnaires tended to load on the same genetic factors, suggesting, to some extent, genetic homogeneity of the items derived from the same questionnaire. Neuroticism was an exception, since part of the items mainly loaded on $G_{-}$NERVOUS, and the other items mainly on factor G_MOODY (analogous to an earlier investigation of the neuroticism personality trait ${ }^{192}$ ). 
Note however that the genetic factors were not exclusive to sets of items from a single questionnaire, i.e., although items derived from the same questionnaire tended to load on the same factors, these genetic factors generally also included items derived from different questionnaires. For instance, virtually all recent anxiety and recent depression items loaded strongly on two genetic factors, one of which they shared with one another. Interestingly, the prolonged anxiety and depression items all loaded onto a single and separate factor, distinguishing them from recent complaints (see Methods and Supplementary Information for more extensive descriptions of the clusters).

\section{Cluster-level SNP-based heritability}

After conducting GWAS on all 7 cluster scores, we applied LDSC to compute SNP-based heritability $\left(h^{2}{ }_{S N P}\right)$ for each cluster (Supplementary Table 10). Clusters with a higher $h^{2}{ }_{S N P}$ are assumed to be more suited for subsequent gene identification. For the theoretical scores $h^{2}{ }_{S N P}$ ranged from $5.5 \%(A N X)$ to $8.1 \%(N E U)$. The genetic factors showed similar variation, with $h^{2}{ }_{S N P}$ ranging from $3.9 \%\left(G_{-} A N X-D E P_{P R O}\right)$ to $8.6 \%\left(G_{-} M O O D Y\right)$. The average $h^{2}{ }_{S N P}$ of the genetic scores was somewhat higher $\left(6.9 \%, 7.9 \%\right.$ if the $h^{2}{ }_{S N P}$ of $G_{-} A N X-D E P_{P R O}$, whose items were not present in the theoretical clusters, was excluded) than that of the theoretical scores $(6.6 \%)$. Interestingly, especially the $h^{2}$ SNP of the genetic scores G_NERVOUS $(8.1 \%)$ and G_MOODY (8.7\%) were considerable, while the $h^{2}{ }_{S N P}$ of $G \_A N X-D E P_{R E C}$ was somewhat lower $(6.9 \%)$ and the $h^{2}{ }_{S N P}$ of the factor representing prolonged complaints, $G_{-} A N X-D E P_{P R O}$, being lowest (3.9\%). 


\begin{tabular}{|c|c|c|c|c|c|c|c|c|c|}
\hline \multirow[t]{2}{*}{ Cluster } & \multirow[t]{2}{*}{ Description } & \multirow[t]{2}{*}{$\mathrm{N}$} & \multicolumn{4}{|c|}{ Genetic clusters } & \multicolumn{3}{|c|}{ Theoretical clusters } \\
\hline & & & G_NERVOUS & G_MOODY & $\begin{array}{l}\text { G_ANX- } \\
\text { DEP }_{\text {REC }}\end{array}$ & $\begin{array}{l}\text { G_ANX- } \\
\text { DEP PRO }\end{array}$ & ANX & DEP & NEU \\
\hline G_NERVOUS & Nervous \& anxious & 126,412 & 0.081 & 0.80 & 0.78 & 0.75 & 0.86 & 0.59 & 0.94 \\
\hline G_MOODY & Moody & 126,532 & 0.66 & 0.087 & 0.92 & 0.70 & 0.86 & 0.88 & 0.88 \\
\hline G_ANX-DEP REC & Recent anxious depression & 126,531 & 0.74 & 0.73 & 0.069 & 0.76 & 0.95 & 0.93 & 0.76 \\
\hline G_ANX-DEP PRO & Prolonged anxious depression & 109,990 & 0.28 & 0.48 & 0.23 & 0.039 & 0.87 & 0.78 & 0.70 \\
\hline ANX & Sum of 7 GAD-7 items & 126,414 & 0.73 & 0.58 & 0.85 & 0.22 & 0.054 & 0.82 & 0.78 \\
\hline DEP & Sum of 8 items from the PHQ-9 & 126,552 & 0.52 & 0.68 & 0.82 & 0.21 & 0.67 & 0.063 & 0.63 \\
\hline NEU & Sum of all 12 items from the EPQ-R-S & 126,382 & 0.77 & 0.74 & 0.43 & 0.21 & 0.39 & 0.36 & 0.081 \\
\hline
\end{tabular}

Note Table 4.1. Phenotypic (lower triangle) and genetic (upper triangle) correlations between the 4 genetically derived clusters and the 3 theoretically derived cluster scores. The diagonal features the $h_{S N P}^{2}$ of each cluster. Genetic correlations and $h_{S N P}^{2}$ were computed using LDSC. 


\section{Genetic correlations between clusters}

To examine how genetically distinct the theoretical and genetic scores were, we calculated the genetic correlations $\left(r_{g}\right)$ between all sum scores (theoretical scores and genetic scores) using LDSC. The $r_{g}$ gives an indication of the extent to which the genetic signal observed for cluster A is similar to that in cluster B (i.e., the extent to which SNP effects are correlated), and is thus taken as an indication of the extent to which the traits are influenced by the same genes.

Genetic correlations between all sum scores were high on average (median $r_{g}=0.78$; Table 3.1; Supplementary Fig. 6; Supplementary Table 11). The overall median $r_{g}$ was very similar to the median within the theoretical scores (median $r_{g}=0.78$ ) and the genetic scores (median $r_{g}=0.77$ ), indicating that within-domain $r_{g}$ 's are not necessarily higher than cross-trait $r_{g}$ 's. However, there did appear to be a distinction between nervous/anxious items and depression items, as the $G_{-}$NERVOUS factor had a notably lower genetic correlation with DEP $\left(r_{g}=0.59\right)$, suggesting that although anxious or nervous worrying is part of depression (as is obvious from the clustering from all depression and anxiety items in the $G_{-} A N X-D E P_{R E C}$ factor, and from the still substantial genetic correlation of 0.59 between $G_{-} N E R V O U S$ and $D E P$ ), there is another part of depression, i.e., a nonanxious part, that correlates with items referring to depressed mood (as operationalized as $G_{-}$MOODY here; $r_{g}=0.88$ ), rather than anxiousness. The current results fit well with the distinction between worry and depressed affect items in neuroticism ${ }^{192}$, that also showed diverging patterns of correlation with external traits.

Interestingly, the highest $r_{g}(0.95)$ was observed between $A N X$ and $G_{-} A N X-D E P_{R E C}$, although $G \_A N X-D E P_{R E C}$ does not only include the 7 GAD-7 items but also 7 out of the 8 recent depression items (note: the genetic correlation between $D E P$ and $A N X$ was also high: $r_{g}=0.82$ ). This high genetic correlation underscores the strong genetic overlap between symptoms used to measure recent anxiety and depression, and calls into question whether these traits are, on a genetic level, indeed distinguishable.

\section{Cluster-level GWAS findings}

Although gene identification was not a primary goal of the current study (i.e., robust gene identification for complex psychological traits typically requires larger $\mathrm{N}$ ), as an illustration, we briefly discuss and compare findings from GWAS between the theoretical clusters and the genetic clusters. GWAS identified 55 genome-wide significant $\left(P<5 \times 10^{-8}\right)$ SNPs for the theoretical scores and 46 for the genetic scores, 5 of which overlapped with each other (Supplementary Table 12). Notably, no GWS SNP associations were identified for $G_{-} A N X-D E P_{P R O}$, and only 1 for $A N X$.

Using FUMA $^{148}$, on online platform for functional mapping and annotation, we mapped the total of 101 GWS SNPs to 14 non-overlapping genomic risk loci across the four genetic and three theoretical scores ( 8 loci for the theoretical scores and 8 for the genetic scores, 2 of which overlapped; Supplementary Table 13; Supplementary Figs. 7-13; see Supplementary Fig. 14 
for Manhattan and Q-Q plots of the 5 phenotypic clusters). Despite the substantial genetic correlations between all clusters, the GWAS results suggest partially distinct, and relatively unique, genetic signals for each cluster (Supplementary Fig. 15).

To investigate whether the genetic signals for the theoretical scores and the genetic clusters are distinct on the gene-level as well, we used FUMA ${ }^{148}$ to map SNPs to genes, and conducted genebased analysis using MAGMA ${ }^{150}$. In total, we identified 67 unique genes for the theoretical scores and 64 for the genetic clusters, of which 10 overlapped (Supplementary Tables 14, 15). Compared to previously reported genes for anxiety ${ }^{271}$, depression ${ }^{40}$ and neuroticism ${ }^{272}$ scales, 20 genes were novel for (and unique to) the genetic clusters, whereas 33 were for the theoretical scores (Supplementary Fig. 15). Gene-set analysis in MAGMA identified a single pathway, defined by genes predominantly expressed in cerebellar brain tissue, that was significantly associated with $G \_N E R \operatorname{VOUS}\left(P=6.72 \times 10^{-6}\right)$.

\section{Genetic correlations to external traits}

In addition to genetic correlations between the various internalizing scores, we also examined patterns of genetic correlations of the theoretical and genetic scores with a set of 22 external psychiatric and health-related outcomes (Fig. 4.2; Supplementary Tables 16, 17). Patterns were largely similar for all genetic scores and theoretical scores, with strong positive genetic correlations evident for mood and anxiety disorders, negative correlations with subjective well-being, and little to no correlation with anthropometric measures. However, there are a few exceptions to note.

$G$ ANX-DEP PRO demonstrated substantially higher positive genetic correlations with schizophrenia and bipolar disorder than any of the other cluster scores. Interestingly, the $r_{g}$ of $G_{-} A N X-D E P_{P R O}$ (based on the prolonged anxiety and depression symptoms) with major depression was substantially higher $\left(r_{g}=0.96\right)$ and with neuroticism was substantially lower $\left(r_{g}=0.66\right)$ compared to those of other genetic clusters and theoretical scores $\left(r_{g}<0.78\right.$, and $r_{g}>0.77$, respectively). There were also multiple distinctions between the two clusters that contained different subsets of the $N E U$ items (despite both clusters also including other items), corroborating earlier results of neuroticism subclusters ${ }^{192}$. For instance, significant positive correlations for ADHD and BMI, and a negative correlation for alcohol intake were observed only for G_MOODY, whereas positive correlations with bipolar disorder and anorexia nervosa were seen only for $G_{-}$NERVOUS. None of those correlations were observed at the level of the full $N E U$ score. 


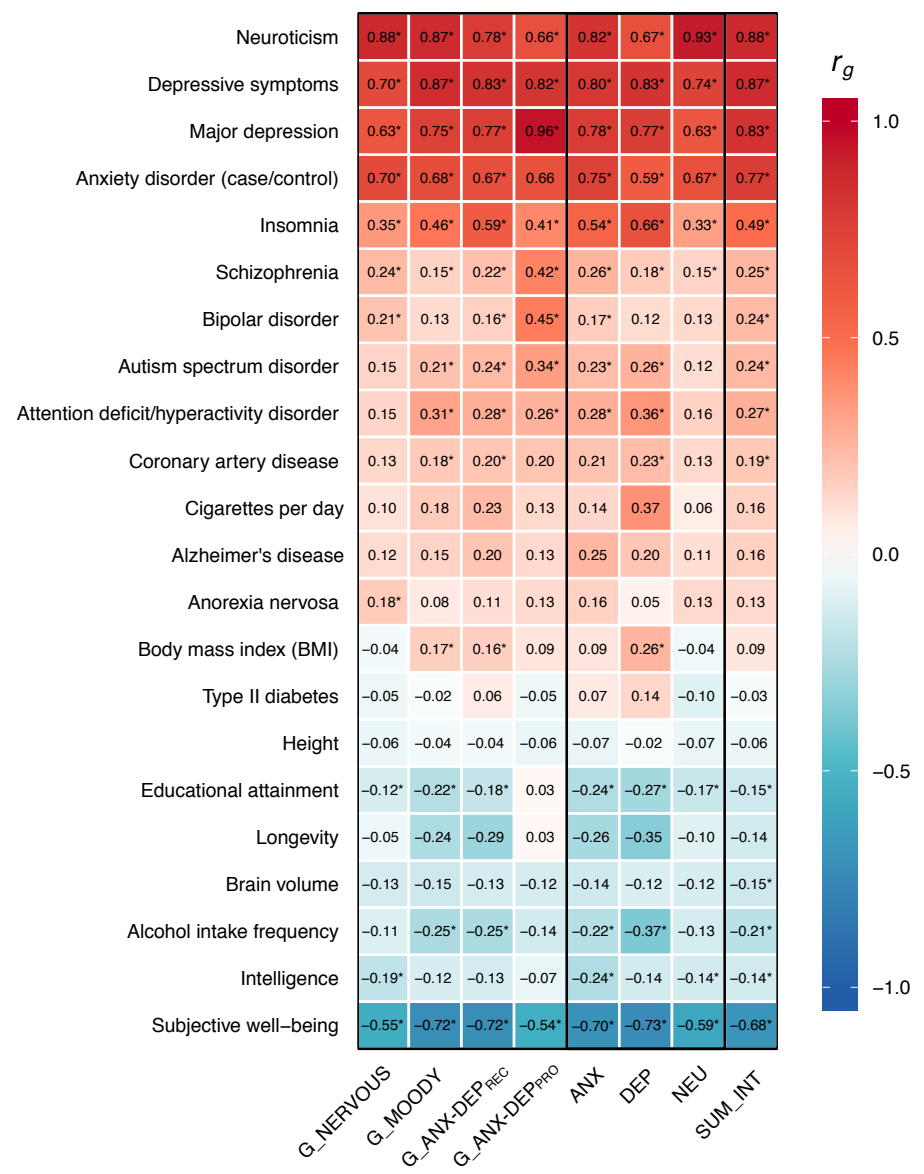

Figure 4.2. Heatmap of genetic correlations between the clusters and external traits.

Genetic correlations of 8 internalizing clusters scores (4 genetic, 3 scale scores, 1 overall internalizing sum score) with 22 psychiatric and health-related traits and diseases for which largescale summary statistics were available (Supplementary Tables 16, 17). Red and blue indicate positive and negative genetic correlations, whereas hue indicates the strength of the genetic correlations. Asterisks indicate genetic correlations for which the $P$ value was lower than the Bonferroni corrected threshold $\left(P<1.75 \times 10^{-4}(0.05 /(13 \times 22))\right.$.

\subsection{Discussion}

The current study aimed to study the genetic validity of diagnostic constructs. Typically, symptoms from a diagnostic interview, or items on a psychological questionnaire, are summed or otherwise aggregated (e.g. case-control status), and this composite score is subsequently used in genetic 
analysis. Here, we studied whether symptoms that are traditionally 'assigned' to the theoretically distinguished constructs anxiety, depression or neuroticism do indeed cluster together on a genetic level.

\section{Summary of main findings}

In a symptom-level analysis of internalizing disorders (anxiety disorders and depression) and a related personality trait (neuroticism) in over 100,000 adults, we found that 34 distinct internalizing symptoms clustered in four genetically homogeneous sets. Through genetic investigation of these genetically derived symptom clusters and comparison with traditional diagnostic scores, we have gained a number of insights into the nature of internalizing disorders that can be used to understand their genetic etiology. Most generally, we conclude that the traditionally distinguished internalizing constructs are not necessarily distinguishable on the genetic level.

First, there appear to be broad, non-specific, cross-disorder genetic influences underlying many facets of the internalizing dimension. Factor analysis of the genetic correlations between the symptoms resulted in a relatively noisy structure with many cross-factor loadings, particularly of the anxiety symptom items. These results are consistent with the widely observed comorbidity of MDD and $\mathrm{ADs}^{105}$, as well as with twin studies demonstrating the genetic overlap across these diagnostic constructs ${ }^{136,137,144}$. A notable exception to this trend was the separation of the neuroticism scale into two distinct factors (irritability/moodiness versus worry/nervousness), as reported earlier in an item-level study of neuroticism ${ }^{192}$. Since we studied the broader internalizing spectrum, these findings suggest that the genetic influences on these moodiness/nervousness dimensions are not unique to neuroticism, but also encompass anxiety and depression symptomatology.

Second, there also appear to be unique genetic influences (e.g., associated genes) that are specific to certain aspects of the internalizing spectrum, whether these aspects are defined by homogeneous symptom clusters or more traditional diagnostic boundaries. Consistent with previous work showing genetic heterogeneity between symptoms of disorders like $\mathrm{MDD}^{93}$, there were two distinct genetic factors underlying the NEU scale and some anxiety/depression items loaded differently on these two clusters. We found unique genomic loci significantly associated with almost every genetic cluster and theoretical sum score, identifying a number of novel genes.

Further, the genetic correlations among cluster and theoretical scores were, although high overall, almost all significantly less than unity. This suggests that, even though there is high genetic overlap, the clusters do differ from one another with respect to their genetic signal. There were also unique patterns of genetic correlations with external domains of psychological and physical functioning. Despite being represented by a single genetic factor, recent anxiety and depression symptoms showed somewhat different patterns of genetic correlations with external phenotypes when they were separate scale scores versus combined in a cluster, indicating that there may be some benefit to genetic analysis of these dimensions separately. In addition, we observed 
differences in strength of genetic correlations to a variety of psychiatric disorders between recent and prolonged anxiety/depression symptoms, suggesting that it may be relevant to stratify patients by the duration of symptoms in future genetic studies.

\section{Limitations}

An important limitation of the current study is the sample size, which is low according to modern GWAS standards (e.g., identification of significant loci for anxiety and depression required much more than 100,000 participants). Although we were able to contrast the genetic scores to the theoretical scores using genetic correlation analysis, power was generally too low for robust gene identification. The fact that the majority of previously reported genes for anxiety, depression and neuroticism was not identified in the current analysis is likely to be explained by large differences in sample size (e.g., the depression meta-analysis by Howard et al. included over 800,000 individuals, whereas the maximum $\mathrm{N}$ in the current study was 126,552). Construction of cluster scores that are, genetically, more homogeneous than their traditional counterparts (i.e., theoretical scores) is expected to increase statistical power ${ }^{85}$. However, individual genetic effects are still expected to be very small, requiring large $\mathrm{N}$ to detect. For instance, in a previous study on the genetic homogeneity of items underlying neuroticism ${ }^{192}$ we found that virtually no genome-wide significant SNPs were identified when analyzing $\sim 110,000$ individuals, but the robustness and magnitude of the genetic signal increased sharply when analyzing $\sim 380,000$ individuals.

\section{A dual pathway approach to studying the genetics of psychological traits}

The current study provides empirical evidence for the proposition that issues of comorbidity and/or genetic heterogeneity underlie some of the challenges in gene identification for internalizing disorders. Our results support, from a molecular genetic perspective, the existence of both phenomena and emphasize the importance of careful consideration of trait definitions used in genetic studies. The fact that unique genomic loci were observed for the genetic clusters, and patterns of genetic correlations with external traits varied between genetic clusters and theoretical scores, demonstrates the extent to which results from genetic studies can be influenced by the choice of operationalization of the phenotype. We firmly believe that a symptom-level approach may be beneficial not only for studying the genetics of internalizing disorders, but also to shed light on the genetics of other psychiatric disorders and psychological traits. For instance, the distinction of two separate symptom dimensions within ADHD, that are differentially associated to psychopathology, cognitive control and other external correlates ${ }^{273}$, warrants investigation of the genetic homogeneity of this disorder.

In the current study we examined how reducing genetic heterogeneity, by means of constructing genetically more homogeneous clusters, affects GWS results. However, it's important to note that also within these relatively homogeneous clusters, groups of individuals may exist with diverging 
symptom profiles. In order to improve our understanding of underlying biology, it might be necessary to solve this problem as well. One approach could be to first, within a genetically homogeneous cluster, identify subgroups of individuals that show similar symptom profiles, and then test whether those subgroups are genetically more similar compared to other subgroups within the same cluster.

We conclude that genetically homogeneous symptom clusters alone will not provide the key to unlock new successes in gene identification, given that the heritability estimates and number of identified genomic loci for genetically homogeneous symptom clusters were on par with that of traditional scale sum scores. However, eventually it's not the mere number of associated loci that will advance our understanding, but the extent to which these loci implicate specific identifiable biological pathways that can in turn be used for e.g. medicine development. A symptom-level approach can provide a more nuanced understanding of genetic etiology that complements brute force approaches that achieve statistical power by increasing sample size through "minimal phenotyping" (i.e., reliance on self-reported responses on one or two questions instead of full diagnostic criteria ${ }^{127}$ ). A dual pathway approach, featuring both minimal versus fine phenotyping strategies, will be important to consider in future research.

\subsection{Methods}

\section{UK biobank sample}

All genetic analyses in the current study were conducted using data from the UK Biobank (UKB). The UKB is a large population-based study, comprising information on hundreds of traits as well as genome-wide genotype data on approximately 500,000 individuals ${ }^{203}$. In addition to the initial touchscreen survey, presented to virtually all participants, an online mental health questionnaire (MHQ) was completed by 157,366 individuals at a later time point ${ }^{36}$. The MHQ was added with the aim to provide more detailed information on mental disorders such as depression, anxiety and mania, and was therefore of particular interest for the current study. Although individuals who completed the MHQ had, on average, higher socioeconomic status than the general population, prevalence of self-reported diagnoses in the UKB data corresponded to population estimates observed in the health survey for England (HSE), a large annual survey ${ }^{36}$.

All procedures within the data collection process of the UKB were in accordance with the World Medical Association for medical research. Ethical approval was granted to the UKB by the National Research Ethics Service Committee North West-Haydock (reference 11/NW/0382). Our study was conducted under UKB application number 16406. 


\section{Phenotypes}

\section{Measurement selection process}

Our initial data set comprised a broad selection of 124 items, spanning 6 categories, from the UKB that were related to all internalizing disorders (see Supplementary Table 1; Supplementary Fig. 1). All data were collected by the UKB, either via touchscreen survey administration in one of the assessment centers or through the online mental health follow-up questionnaire $\left(\mathrm{MHQ}^{36}\right)$.

Items were filtered based on whether they could be considered 'symptoms' resembling diagnostic criteria. GWAS was performed on those traits for which data of at least 50,000 individuals (or for case/control phenotypes: 10,000/10,000 individuals) from European descent were available in the UKB. Details on phenotype selection and analysis of these traits are described elsewhere ${ }^{268}$. All GWAS summary statistics are available from the GWAS ATLAS ${ }^{268}$ resource (URLs).

Subsequently, we excluded items that were only administered to a subsample of participants based on their response to diagnostic screening items (skip logic; e.g., only participants responding 'yes' to the question whether they ever experienced prolonged feelings of sadness or depression were presented with follow-up questions about symptoms during this depressed period). In addition, we excluded items for which the SNP-based heritability $\left(h^{2}{ }_{S N P}\right)$ was $\sim 0$, or for which the standard error of $h^{2}{ }_{S N P}$ was extremely large ('recent thoughts of suicide or self-harm' (UKB field ID: 20513) and 'manic/hyper for 2 days' (4642)).

Lastly, we excluded three positive valence items ('happiness' (4526), 'general happiness' (20458) and 'belief own life is meaningful' (20460)) and the insomnia item (1200). The "happiness" and "general happiness" items correlated to such an extent (phenotypic $r=0.53$, genetic $r_{g}=1.01$ ) that we initially selected only the happiness item (4526) for the factor analysis to avoid ending up with a factor structure determined by items that are virtually duplicates. Yet, the remaining positive valence items (4526 and 20460) also tended to cluster together and form a specific factor in more extended factor solutions (i.e., $>3$ factors). Similarly, the insomnia item (1200: "Do you have trouble falling asleep at night or do you wake up in the middle of the night?") correlated considerably with one sleep item included in the depression scale (i.e., the Patient Health Questionnaire 9-question version; PHQ-9270; 20517: “Trouble falling or staying asleep, or sleeping too much"; $r=0.33 ; r_{g}=.82$ ), and much less strongly with the other items. In more extended factor solutions (i.e., $>3$ factors), these items also tended to form a specific factor, while the PHQ-9 sleep item in addition showed considerable correlation with the other PHQ-9 items. Exclusion of the 3 positive valence items and the insomnia item resulted in a much cleaner and more interpretable factor structure (i.e., a solution without factors representing only 2 highly correlated items). Consequently, the main analyses reported here are based on a selection of 34 internalizing items (Supplementary Tables 1, 2).

\section{Description of internalizing-related measures in UKB}

An overview of all 34 items included in the current analyses is provided in Supplementary Table 2 . 


\section{Current anxiety}

The MHQ included 7 items measuring experienced anxiety in the last two weeks. Items were derived from the Generalized Anxiety Disorder 7 (GAD-7 ${ }^{86}$ ), a brief anxiety scale based on symptom criteria for generalized anxiety disorder described in the Diagnostic and Statistical Manual of Mental Disorders, Fourth Edition (DSM-IV ${ }^{274}$ ). Participants were requested to indicate the extent to which they experienced certain complaints (e.g., "Feeling nervous, anxious or on edge", "') over the last two weeks by choosing one of four categories ('Not at all', 'Several days', 'More than half the days', 'Nearly every day'). Endorsement rates for all ordinal items are reported in Supplementary Table 2. The GAD-7 has been shown to have high test-retest reliability (intraclass correlation $=0.83$ ), and outcomes are strongly related to several domains of functional impairment and self-reported disability days ${ }^{86}$.

\section{Current depression}

In order to measure the absence or presence, and severity, of current depression, the MHQ included items from the Patient Health Questionnaire 9-question version (PHQ-9 ${ }^{86,275}$ ). The PHQ-9 is a selfreport questionnaire based on the more extensive PRIME-MD ${ }^{276}$, and features 9 items that reflect the criteria for major depressive disorder as defined in the DSM-IV ${ }^{274}$. Participants were asked to report how often they experienced a number of problems (e.g., "Feeling down, depressed, or hopeless", "Little interest or pleasure in doing things") over the course of the last 2 weeks. Response categories were similar to the current anxiety items, ranging from 'Not at all' to 'Nearly every day'. We excluded the most extreme item of the PHQ-9 (concerning "recent thoughts of suicide or selfharm") from further analysis, because of its extremely low prevalence (95.7\% of the individuals responded with 'Not at all', and only $0.8 \%$ of the individuals responded 'More than half the days' or 'Nearly every day'): the low prevalence had unfavorable consequences for the estimation of the LDSC $h^{2}{ }_{S N P}$ of this item and the LDSC genetic correlation of this item to all other items, invalidating it for subsequent analyses. The correlation between PHQ-9 scores and depressive symptoms as recorded by mental health professionals was $r=0.84$, validating the PHQ- $9^{275}$.

\section{Lifetime anxiety screeners}

In addition to the current anxiety items, two items in the MHQ assessed lifetime anxiety. Participants were asked whether they 1) ever had a period lasting one month or longer when they felt worried, tense or anxious most of the time and 2) if there ever was a time when they worried a lot more than most people would in the same situation. Response categories were 'Yes', 'No', 'Do not know' or 'Prefer not to answer'. The questions were modified from the Composite International Diagnostic Interview Short-Form (CIDI-SF ${ }^{277}$ ).

\section{Lifetime depression screeners}

We included four items aimed at examining lifetime depression problems in the current study. Two of the items focused on sad or depressed mood, and two other items on loss of interest in normal 
activities ('anhedonia') for a prolonged period of time. Whereas two items included in the baseline touchscreen survey of the UKB assessed whether participants had ever experienced depressed mood or anhedonia for at least a week, the two items in the MHQ, administered approximately 711 years after the initial visit, inquired about these problems lasting two weeks or more in a row. Despite the apparent overlap in item content, correlations between these measures were sufficiently low to merit inclusion of both sets in the analyses $(r=0.65-0.72)$. Participants were presented with the following response options: 'Yes', 'No', 'Prefer not to answer'. Questions were modified from the Composite International Diagnostic Interview Short-Form (CIDI-SF ${ }^{277}$ ).

\section{Neuroticism items}

Neuroticism was measured via the touchscreen survey in UKB assessment centers, operationalized through the 12-item Eysenck Personality Questionnaire, Revised Short Form (EPQ-R-S ${ }^{88}$ ). Participants were required to respond 'Yes' or 'No' to 12 statements about their usual temperament and behavior (e.g., "Would you call yourself tense or 'highly strung'?”, “Are you a worrier?”).

\section{Bipolar screening item}

We included one additional question from the touchscreen survey, assessing whether participants ever had a period of at least two days when they were highly irritable or argumentative. Although no external sources of validation were supplied for this item, we found it to be highly correlated to the irritability items from the neuroticism and anxiety questionnaires $\left(r \sim 0.42 ; r_{g}{ }^{\prime} \mathrm{s} \sim 0.75\right)$.

\section{Genotyping, imputation \& quality control}

For the current study, we used the imputed genotype data released by the UKB in March 2018 (version 3). A detailed overview of data collection and quality control (QC) procedures can be found elsewhere ${ }^{203}$. Briefly, the UKB has collected genotype data from 489,212 individuals, using two custom-designed genotype arrays that share approximately $95 \%$ of marker content (Applied Biosystems $^{\mathrm{TM}}$ UK BiLEVE Axiom ${ }^{\mathrm{TM}}$ Array by Affymetrix and the Applied Biosystems ${ }^{\mathrm{TM}}$ UK Biobank Axiom ${ }^{\mathrm{TM}}$ Array). The genotype data was then imputed by the UKB team with IMPUTE4, using a reference panel merging the $\mathrm{UK} 10 \mathrm{~K}^{278}$ haplotype data with data from the Haplotype Reference Consortium ( $\mathrm{HRC}^{279}$ ). After imputation and quality control the genotype data comprised 487,422 individuals and 92,693,895 genetic variants.

We further processed the data using an in-house GWAS pipeline. All imputed variants were converted to hard-calls, applying a certainty threshold of 0.9. In an additional QC step, we excluded SNPs with a low imputation quality (INFO $<0.9)$, a very low minor allele frequency $(\mathrm{MAF}<0.0001)$ and high missingness $(>0.05)$. We also excluded a number of indels that were located in the same position (i.e., same chromosome and base pair position), resulting in a total of 9,203,453 SNPs used

in the analyses (given differences in available sample size and associated MAF filtering during analysis, the exact number of variants that is used in individual analyses may vary across phenotypes). 
All analyses described in the current study were restricted to participants of European ancestry. Principal components (PC's) from the 1000 Genomes reference populations ${ }^{174}$ were projected onto the genotype calls available in the UKB data. For every individual, the Mahalanobis distances between their projected principal component score and the average scores of the populations in the 1000 Genomes sample 280 were computed. Only participants for whom the score was closest to the average of the European sample, and for whom the Mahalanobis distance was less than 6 standard deviations, were included in the analysis. Finally, participants were excluded who 1) had withdrawn their consent, 2) were related according to the UKB team (subjects with most inferred relatives, $3^{\text {rd }}$ degree or closer, were removed until no related subjects were present), 3) reported a gender that did not match their genetic gender, or 4) showed sex-chromosome aneuploidy.

\section{Exploratory factor analysis of item-level data}

General methodology of factor analysis

We performed exploratory factor analysis on the data of the 34 items, aiming to obtain sets of items that cluster together (see Supplementary Fig. 5 for a flow chart of analyses conducted in the current study). All factor analyses were performed in $R$. Both the genetic factor structure and the phenotypic factor structure was explored using the genetic and phenotypic correlation matrix as input, respectively. We used maximum likelihood (ML; Geomin oblique rotation).

We considered a number of criteria in determining the number of factors that captured most of the variance in the individual items. First of all, we focused on factor models with only factors whose Eigenvalue were $>1$ (Supplementary Figs. 3, 4). Secondly, we preferred factor models with simple structure (i.e., no or as few as possible items loading on multiple factors). Third, factor loadings should be $>.3$. Finally, we considered the extent of increase in model fit with additional factors (i.e., the change in TLI, BIC, RMS, RMSEA and total proportion of variance explained by the factors), and preferred interpretable factor solutions (i.e., the choice of the optimal number of factors was also guided by interpretability of the factor solution).

\section{Genetic factor analysis}

Using linkage disequilibrium score regression $\left(\operatorname{LDSC}^{116,146}\right)$ we computed genetic correlations $\left(r_{g}\right.$ 's) between all 34 internalizing items (Supplementary Fig. 2; Supplementary Table 4). LDSC requires GWAS summary statistics as input, and $r_{g}$ 's are unbiased by sample overlap. Precomputed LD scores based on the Europeans in the 1000 genomes reference data were obtained online (https://data.broadinstitute.org/alkesgroup/LDSCORE/). Only HapMap3 SNPs were included in the analysis. Genetic correlations were deemed significant if the $P$ value was lower than the Bonferroni corrected threshold $\alpha=0.05 /(34 \times(34-1) / 2)=8.91 \times 10^{-5}$.

We used Genomic SEM ${ }^{281}$ to obtain the smoothed genetic variance-covariance matrix between the 34 items, which we then transformed to a correlation matrix using the $\operatorname{cov} 2 \operatorname{cor}$ function in $R$. Note that smoothing of the genetic variance-covariance matrix (genomic SEM uses the $R$-package near $P D$ for smoothing) is often required because the initial matrix is sometimes not fully positive 
definite because a) the matrix is built up element-by-element, and b) the individual estimates (variances, covariances) have considerable standard errors (i.e., when the $h^{2}{ }_{S N P}$ of individual items is small, the $r_{g}$ between these items cannot be estimated very reliably). We note that the smoothed and unsmoothed matrices are extremely similar.

Simple structure was hard to obtain in the genetic factor analysis. Particularly the anxiety items often loaded on more than 1 factor. The 4-factor model proved to be most interpretable (after 4 factors, the factor loading pattern became scattered and uninterpretable), and most items, bar the anxiety items, loaded on only 1 factor (see Supplementary Table 7 for the solutions of the first 7 factors).

The first genetic factor (prefix ' $G$ ') comprised mostly a subset of the neuroticism items related to nervousness, as well as all anxiety items. Hence, this factor was labeled G_NERVOUS. The second factor, labeled G_MOODY, included all three 'irritability' items as well as a subset of depression and neuroticism items relating to negative mood. Virtually all current anxiety and depression items loaded strongly on the third factor, $G \_A N X-D E P_{R E C}$. The fourth factor $\left(G \_A N X\right.$ $\left.D E P_{P R O}\right)$ included all 6 of the lifetime anxiety and depression items. More extensive descriptions of each cluster are provided in Supplementary Table 10.

\section{Creating clusters}

In order to be able to compare genetic signal of the conventional operationalization of disorders (e.g., for anxiety; sum-score of all anxiety items) to the empirically derived phenotypes from factor analysis, we constructed clusters. The clusters were subsequently used to perform GWAS on. Cluster scores were constructed by summing the scores on the items loading $(>0.3)$ on the same factor. Ordinal items were dichotomized prior to this step (collapsing the latter three response categories ('Several days', 'More than half the days' and 'Nearly every day') into an indicator of experiencing vs. not experiencing each symptom), to assure that all items contributed equally to the cluster score. As the ordinal items had highly skewed patterns of response (mean scores for the ordinal items, $\min =1.00, \max =4.00$ ranged from 1.08 to 1.72 ), this dichotomization did not result in substantial loss of information.

Aside from the 4 genetic and 5 phenotypic clusters (see Supplementary Information) we computed 4 theoretical scores. These were computed by adding 1) all items (overall internalizing sum-score; SUM_INT) and 2) all items that originate from the recent anxiety (ANX), recent depression (DEP) and neuroticism (NEU) questionnaires, respectively. The latter three clusters correspond to the conventional operationalization of the three traits using psychometrically validated scales. Since our primary focus is on studying whether items that are typically summed into scale scores are clustering together on the genetic level, we limit the discussion of results to the 3 theoretical scores and the 4 genetic clusters (results on the phenotypic clusters are, however, included in supplementary tables and in the Supplementary Information). 


\section{Cluster-level GWAS}

We ran GWAS on all 13 clusters ( 3 theoretical scores, 4 genetic clusters, 5 phenotypic clusters and 1 overall internalizing sum score) using a linear regression model of additive effects in PLINK $1.9^{175,176}$. Prior to analysis, all cluster scores were standardized to have mean 0 and a variance of 1 . The model included sex, age, genotyping array, Townsend deprivation index (a measure of socioeconomic status), UKB assessment center and 10 genetic PC's as covariates. The genetic principal components were computed with FlashPCA2 282 in the post-QC subset of unrelated European individuals, using 145,432 independent $\left(r^{2}<0.1\right)$, common (MAF $\left.>0.01\right)$ and genotyped SNPs (INFO=1). The maximum per-SNP N ranged from 75,274 (P_ANX-DEP PRO) to 126,552 (DEP; see Supplementary Table 10).

\section{Cluster heritability and genetic correlations}

\section{SNP-based heritability}

Using $\operatorname{LDSC}^{146}$ we computed the SNP-based heritability $\left(h^{2}{ }_{S N P}\right.$; the proportion of phenotypic variance that can be explained by all SNPs in the GWAS) for the 13 primary clusters (Supplementary Table 10). Precomputed LD scores based on the Europeans in the 1000 genomes reference data were obtained online (see URLs). Only HapMap3 SNPs were included in the analysis.

\section{Genetic correlations}

Genetic correlations $\left(r_{g}\right.$ 's) between the clusters were calculated using $\operatorname{LDSC}^{116,146}$. Since the clusters are expected to correlate, we do not provide $P$ values indicating departure from 0 . However, the standard errors for the $r_{g}$ 's can be used to determine whether the genetic correlation is statistically different from unity (i.e., where a genetic correlation of 1 implies complete overlap in the genetic factors influencing clusters). In order to correct for multiple testing, we applied a stringent Bonferroni significance threshold of: $0.05 /((13 \times 12) / 2)=6.41 \times 10^{-4}$. Furthermore, we computed, in the same manner, $r_{g}$ 's between the clusters and 22 external traits and disorders using publicly available summary statistics (Supplementary Tables 16, 17).

\section{Genomic risk loci}

FUMA $^{148}$, an online platform to annotate and interpret GWAS results, was used to determine genomic risk loci. First, independent significant SNPS were defined as being genome-wide significant (GWS, $P<5 \times 10^{-8}$ ) and independent from each other at $r^{2}<0.6$. Using linkage disequilibrium (LD) information based on a subset of the UKB data, a number of independent significant SNPs that were independent from each other at $r^{2}<0.1$ was labeled 'lead SNP'. Finally, genomic risk loci were established by merging lead SNPs that physically overlapped, or for which the LD blocks were located within $250 \mathrm{~kb}$ from one another. 


\section{Mapping SNPs to genes}

We used three strategies within FUMA ${ }^{148}$ (version 1.3.2) to map all SNPs that were GWS, or in LD $\left(r^{2}>0.6\right)$ with one of the independent significant SNPS, to genes. The first strategy, positional mapping, maps SNPs to genes if they are within $10 \mathrm{~kb}$ from known protein coding genes in the human reference genome assembly (GRCh37/hg19). Secondly, eQTL mapping uses gene expression information (GTEx, Blood eQTL browser and BIOS QTL browser) to map SNPs to genes if the expression of that gene is associated with allelic variation of the SNP. eQTL mapping is based on cis-eQTLs, and can map SNPs to genes that are up to $1 \mathrm{Mb}$ apart. A false discovery rate (FDR) of 0.05 was applied to define significant eQTL associations. The third strategy, chromatin interaction (CI) mapping, uses information on the complex 3D structure of DNA to identify interactions between genomic risk loci and promoter regions of genes. Promoter regions were defined as being $250 \mathrm{bp}$ up- and $500 \mathrm{bp}$ downstream of the transcription start site (TSS). CI mapping does not have a distance boundary, potentially mapping SNPs to distant genes. At this time, FUMA uses Hi-C data of 14 tissue types ${ }^{184}$. It's important to note that a region involved in the interaction may contain several genes, due to the resolution of chromatin interactions (here; 40 $\mathrm{kb})$. We further prioritized genes identified by CI mapping using information on predicted enhancers and promoters in 111 tissue/cell types from the Roadmap Epigenomics Project ${ }^{183}$. Integrating these data resulted in selecting those chromatin interactions for which one region overlapped with predicted enhancer of a gene, whereas the other region overlapped with the predicted promoter of a gene. Analogous to eQTL mapping, an FDR of $1 \times 10^{-5}$ was applied to define significant interactions.

\section{Gene-based analysis}

We performed gene-based analysis in MAGMA ${ }^{150}$ for all theoretical scores and genetic clusters. Gene-based analysis assesses the joint effect of all SNPs located within a gene (including SNPs 2 $\mathrm{kb}$ before and $1 \mathrm{~kb}$ after the TSS), while taking the LD between SNPs into account. GWAS summary statistics were used as input for MAGMA, and we used all 19,427 protein-coding genes from the NCBI 37.3 gene definitions. Gene $P$ values were corrected for multiple testing by dividing the significance threshold $\alpha=0.05$ by the number of genes that were represented by at least one SNP. 


\subsection{Supplementary methods}

\section{Factor analysis on phenotypic correlations}

Due to the nature of the items included in the current study (binary and ordinal response categories) the phenotypic correlation matrix was a mix of tetrachoric and polychoric correlations (see Supplementary Table 3). Based on the scree plot (Supplementary Fig. 3), 1 to 5 factors were required to capture most of the variance in the 34 items. Using a threshold of .3 for the factor loadings, the criterion of simple structure appeared to hold relatively well for all phenotypic factor solutions (except for the 4-factor model). We selected the 5-factor solution (Supplementary Tables 5, 6) based on Eigenvalues, simple structure, interpretability, lack of sparse factors, and a large proportion of item variance accounted for (64\%).

The factor structure of the phenotypic correlations generally reflected the scale and/or measurement time frame by which the items were assessed. The first phenotypic factor (prefix ' $P$ ') consisted mostly of the GAD-7 items, and was labeled $P_{-} A N X_{R E C}$ (recent anxiety; the 'REC' subscript indicates that this concerns recent experiences). The second factor ( $P_{-} D E P_{R E C}$; recent depression) encompassed all eight depression items from the PHQ-9. Interestingly, all 6 items that assess lifetime anxiety or depression (i.e., prolonged problems) loaded strongly on the third factor ( $P \_A N X-D E P_{P R O}$; lifetime anxious depression). The fourth and fifth factors were labeled $P_{-} M O O D Y$ and $P_{-}$NERVOUS, respectively. $P_{-} M O O D Y$, included two of the three 'irritability' items and 6 additional NEU items indicating negative affect/moodiness, while $P_{-}$NERVOUS included NEU items such as nervousness, tension, and worrying. The $P_{-} M O O D Y$ and $P \_$NERVOUS factors coincided with the distinction between two neuroticism clusters reported earlier ${ }^{192}$. Notably, the prolonged lifetime worry item loaded moderately on three factors ( $P \_A N X_{R E C}, P_{-} A N X_{P R O}$, and $\left.P_{-} N E R V E\right)$. More extensive descriptions of each cluster are provided in Supplementary Table 9.

\section{Description of phenotypic clusters}

The five clusters that emerged from EFA on the phenotypic inter-item correlations appear to distinguish well between anxiety and depression, but only when it concerns recently experienced complaints (Supplementary Table 5). Whereas the first cluster, labeled $P_{-} A N X_{R E C}$, comprised all GAD-7 items plus one prolonged anxiety item, the second cluster, $P_{-} D E P_{R E C}$, comprised exclusively the recent depression items. Interestingly, the sharp distinction implied by the first two factors dissolves when considering long term symptoms, i.e., the $3^{\text {rd }}$ phenotypic cluster $\left(P \_A N X\right.$ $\left.D E P_{P R O}\right)$ consists of the six prolonged anxiety and depression items, indicating that, among individuals with lifetime complaints, anxiety and depression very often co-occur. The remaining two clusters ( $P_{-} M O O D Y$ and $P_{-}$NERVOUS), mostly consisting of items from the neuroticism questionnaire, can be interpreted as a moodiness (e.g., irritability, mood swings) and a nervous (e.g., suffering from nerves, excessive worrying) component, respectively. Whereas the 
$P_{-} M O O D Y$ cluster showed high genetic correlations with both $P_{-} A N X_{R E C}\left(r_{g}=0.78\right)$ and $P \_D E P_{R E C}$ $\left(r_{g}=0.77\right)$, the nervous component $\left(P_{-}\right.$NERVOUS $)$only has a high genetic correlation with $P_{-} A N X_{R E C}\left(r_{g}=0.76\right)$, and not with $P_{-} D E P_{R E C}\left(r_{g}=0.35\right.$; see Supplementary Fig. 6). This finding is in line with the genetic correlational pattern between two subclusters of neuroticism and anxiety and depression reported earlier ${ }^{192}$.

\section{Heritability of clusters}

We performed GWAS on each of the 13 clusters that we established (Supplementary Table 9). Quantile-quantile (Q-Q) plots showed minimal to substantial inflation (Lambda GC ranged from 1.06 to 1.19 ) of the $\chi^{2}$ test statistic, depending on the cluster (Supplementary Table 10; Supplementary Figures 7, 8). The LDSC intercepts (range: 1.000 to 1.011) suggested that the observed inflation was due to true polygenicity and large sample size. SNP-based heritability $\left(h_{S N P}^{2}\right)$ varied from a low $4.1 \%\left(P_{-} A N X-D E P_{P R O}\right)$ to a high $8.7 \%\left(G_{-} M O O D Y\right)$, and all were significantly different from zero.

\section{URLs}

FUMA GWAS platform: http://fuma.ctglab.nl/

LD Score regression: https://github.com/bulik/ldsc

Pre-calculated LD-scores: https://data.broadinstitute.org/alkesgroup/LDSCORE/)

MAGMA: https://ctg.cncr.nl/software/magma

MsigDB gene-sets: http://software.broadinstitute.org/gsea/msigdb/index.jsp

GTEx portal: https://www.gtexportal.org/home/

GWAS ATLAS: https://atlas.ctglab.nl/

Genomic SEM: https://github.com/MichelNivard/GenomicSEM

\section{Acknowledgements}

This work was funded by the Netherlands Organization for Scientific Research through the following grants: NWO MagW VIDI 452-12-014 (S.v.d.S.), NWO VICI 435-13-005 (D.P.) Analyses were carried out on the Genetic Cluster computer, which is financed by the Netherlands Scientific Organization (NWO award 480-05-003 to D.P.), VU University (Amsterdam, The Netherlands), and the Dutch Brain Foundation and is hosted by the Dutch National Computing and Networking Services, SurfSARA. This research has been conducted using the UK Biobank resource (application 16406). 



\section{$5 \quad$ Genome-wide meta-analysis of brain volume on 47,316 individuals identifies genomic loci and genes shared with intelligence}

Philip R Jansen ${ }^{1 \dagger}$, Mats Nagel ${ }^{1 \dagger}$, Kyoko Watanabe ${ }^{1}$, Yongbin Wei ${ }^{1}$, Jeanne E Savage ${ }^{1}$, Christiaan A de Leeuw ${ }^{1}$, Martijn $P$ van den Heuvel ${ }^{1,2}$, Sophie van der Sluis ${ }^{1,2 \S}$, Danielle Posthuma ${ }^{1,2 \S^{*}}$

1. Department of Complex Trait Genetics, Center for Neurogenomics and Cognitive Research, Amsterdam Neuroscience, Vrije Universiteit Amsterdam, Amsterdam, The Netherlands

2. Department of Child Psychiatry, Section Complex Trait Genetics, Amsterdam Neuroscience, Vrije Universiteit Medical Center, Amsterdam UMC, Amsterdam, the Netherlands

These authors contributed equally to this work

$\S \quad$ These authors jointly supervised this work

Manuscript under revision with Nature Communications 


\section{Abstract}

The phenotypic correlation between human intelligence and brain volume (BV) is considerable $(\mathrm{r} \approx 0.40)$, and has been shown to be due to shared genetic factors 1 . To further examine specific genetic factors driving this correlation, we present genomic analyses of the genetic overlap between intelligence and BV using genome-wide association study (GWAS) results. First, we conducted a large BV GWAS meta-analysis ( $\mathrm{N}=47,316$ individuals), followed by functional annotation and gene-mapping. We identified 18 genomic loci (14 novel), implicating 343 genes (270 novel) and 18 biological pathways for BV. Second, we used an existing GWAS for intelligence ( $N=269,867$ individuals2), and estimated the genetic correlation ( $\mathrm{rg}$ ) between BV and intelligence to be 0.24 . We show that the rg is partly attributable to physical overlap of GWAS hits in 5 genomic loci. We identified 92 shared genes between BV and intelligence, which are mainly involved in signaling pathways regulating cell growth. Out of these 92, we prioritized 32 that are most likely to have functional impact. These results provide new information on the genetics of BV and provide biological insight into BV's shared genetic etiology with intelligence. 


\subsection{Introduction}

The relation between brain volume (BV) and cognitive ability has been a fundamental question to cognitive sciences for a long time ${ }^{283-286}$. Before the advent of magnetic resonance imaging (MRI), brain volume was typically determined from autopsy records ${ }^{287}$, or inferred by external head size measures. A literature meta-analysis conducted by Vernon et al. $(2000)^{288}$ estimated the correlation between external head measures and intelligence to be around 0.19 . Five years later, a meta-analysis of 24 studies using MRI-based measures of brain volume (with the subtle title; "Big brains are better") found a substantially higher correlation of 0.33 with intelligence ${ }^{289}$. Recently, an exhaustive meta-analysis ${ }^{290}$, including 88 studies comprising 148 samples, was conducted, aiming to readdress the much-debated relation between BV and intelligence. This study found that brain volume and intelligence were, indeed, robustly correlated, albeit lower $(r=0.24)$ than what was reported in the literature. The difference may in part be explained by publication bias, with strong, positive correlations more often being reported than small, non-significant ones. Recently, these findings were supported by yet another large-scale study reporting a correlation of $r=0.19$ between brain volume and fluid intelligence ${ }^{291}$ (referring to problem-solving and logical skills that do not depend on previously acquired knowledge). Interestingly, previous studies did not find evidence for sex differences in the relation between BV and intelligence ${ }^{290}$.

The conservative correlation of $\sim .20$, implies an explained variance of just $4 \%$. This is low and therefore $\mathrm{BV}$ is not a good predictor of intelligence, and vice versa, intelligence is not a good predictor of BV. However, knowing that this correlation is at least partly due to shared genetic factors ${ }^{292}$ suggests that knowledge of which specific genetic factors are involved in both traits may provide at least some insight into the observed variance in both $\mathrm{BV}$ and intelligence. In the current study we utilize large-scale genetic data and advance statistical genetics tools, to examine the genetic factors that underlie the observed correlation.

\subsection{Results}

\section{GWAS meta-analysis of brain volume}

To identify single nucleotide polymorphisms (SNPs) associated with BV, we first performed a GWAS (Supplementary Fig. 1) using data of 17,062 participants from the UK Biobank (UKB ${ }^{293}$ ), with BV estimated from structural ( $\mathrm{T}_{1}$-weighted) magnetic resonance imaging (MRI) by summing total gray and white matter volume, and ventricular cerebrospinal fluid volume ${ }^{283,294}$ (Supplementary Fig. 2). Within the UKB data, we identified 3,610 genome-wide significant $\left(\mathrm{GWS} ; P<5 \times 10^{-8}\right)$ SNPs, tagging 9 independent genomic loci. The SNP-based heritability $\left(h^{2}{ }_{S N P}\right)$ estimated through linkage disequilibrium score regression (LDSC ${ }^{116,204}$; Online Methods) was $35.3 \%$ ( $\mathrm{SE}=4.1 \%$ ). The LDSC intercept of 1.02 , however, suggested that the observed inflation in 
genetic signal is mostly due to polygenicity and unlikely to be driven by population stratification ${ }^{196}$ (see Supplementary Results). The UKB GWAS results were then meta-analyzed with GWAS results from two previously published studies ${ }^{295,296}$ : one on intracranial volume (ICV) from the ENIGMA consortium ${ }^{297}(\mathrm{~N}=11,373)$, the other on head circumference (HC), a proven proxy of $\mathrm{BV}^{298-300}$ (also see Supplementary Methods), from a recent GWAS meta-analysis ${ }^{301}$ of adults and children $(\mathrm{N}=18,881)$. This led to a total sample size of 47,316 unrelated Europeans (Supplementary Fig. 3; Supplementary Methods 1.1-1.2; Supplementary Table 1). LD score regression showed high concordance of SNP associations between the three samples (UKB and ENIGMA: $r_{g}=1.25, \mathrm{SE}=0.20$; UKB and HC-GWAS: $r_{g}=0.75$, SE=0.09; ENIGMA \& HC-GWAS: $r_{g}=0.94, \mathrm{SE}=0.20$; Supplementary Table 2), justifying subsequent meta-analysis. Sample-size weighted fixed-effects meta-analysis was carried out using METAL ${ }^{179}$ (Online Methods) resulting in 24 linkage disequilibrium (LD) independent lead SNPs $\left(r^{2}<0.1\right)$, residing in 18 genomic loci (Fig.5. 1a; Supplementary Table 3; Supplementary Fig. 4), representing 4,155 genome-wide significant SNPs (Fig. 5.1b, Supplementary Table 4) associated with BV. Of these 18 loci, 14 were novel compared to the latest GWAS study of intracranial volume ${ }^{295}$ (2 loci reported in this earlier study were not GWS in the current meta-analysis).

To see if our results were driven by only one of the three samples or were supported by all three, we examined the direction of effect of all genome-wide significant (GWS) SNPs across the three individual cohorts. As expected, we found high concordance: all SNPs that were GWS in the meta-analysis, and that were present in all three cohorts, had the same direction of effect. Of the 4,155 SNPs that were GWS in the BV meta-analysis, just $2.4 \%$ was GWS in at least two of the individual cohorts, however, $75.0 \%$ had a $P$ value $<0.05$ in at least two cohorts (and $21.2 \%$ in all three cohorts). The SNP-heritability $\left(h^{2}{ }_{S N P}\right)$ of BV estimated by LDSC from the meta-analytic results was $21.4 \%(\mathrm{SE}=1.8 \%)$, and the LDSC intercept approximated 1 (1.025, SE=0.009), suggesting that the inflation in test statistics (Lambda $\lambda_{\mathrm{GC}}=1.18$ ) was largely due to polygenicity and not to unaccounted for population stratification ${ }^{146}$. Functional annotation of 4,683 'candidate' SNPs (i.e., SNPs in the risk loci with a GWAS $P$ value of $P<10^{-5}$ and LD $r^{2}>0.6$ with one of the independent significant SNPs; Online Methods) carried out in FUMA ${ }^{148}$ showed that these SNPs were most abundant in intronic $(\mathrm{n}=2,444,52,1 \%)$ or intergenic regions $(\mathrm{n}=751,16.0 \%)$, and 27 $(0.6 \%)$ were exonic nonsynonymous SNPs (ExNS) altering protein structures of 12 genes (Supplementary Fig. 5; Supplementary Tables 5, 6). One gene, $S P P L 2 C$, contained 8 ExNS (all in exon 1, and in the same inversion region). SPPL2C codes for the signal peptide peptidase-like $2 \mathrm{C}$, which plays a role in the degradation of signaling peptides in the brain $^{302}$. 

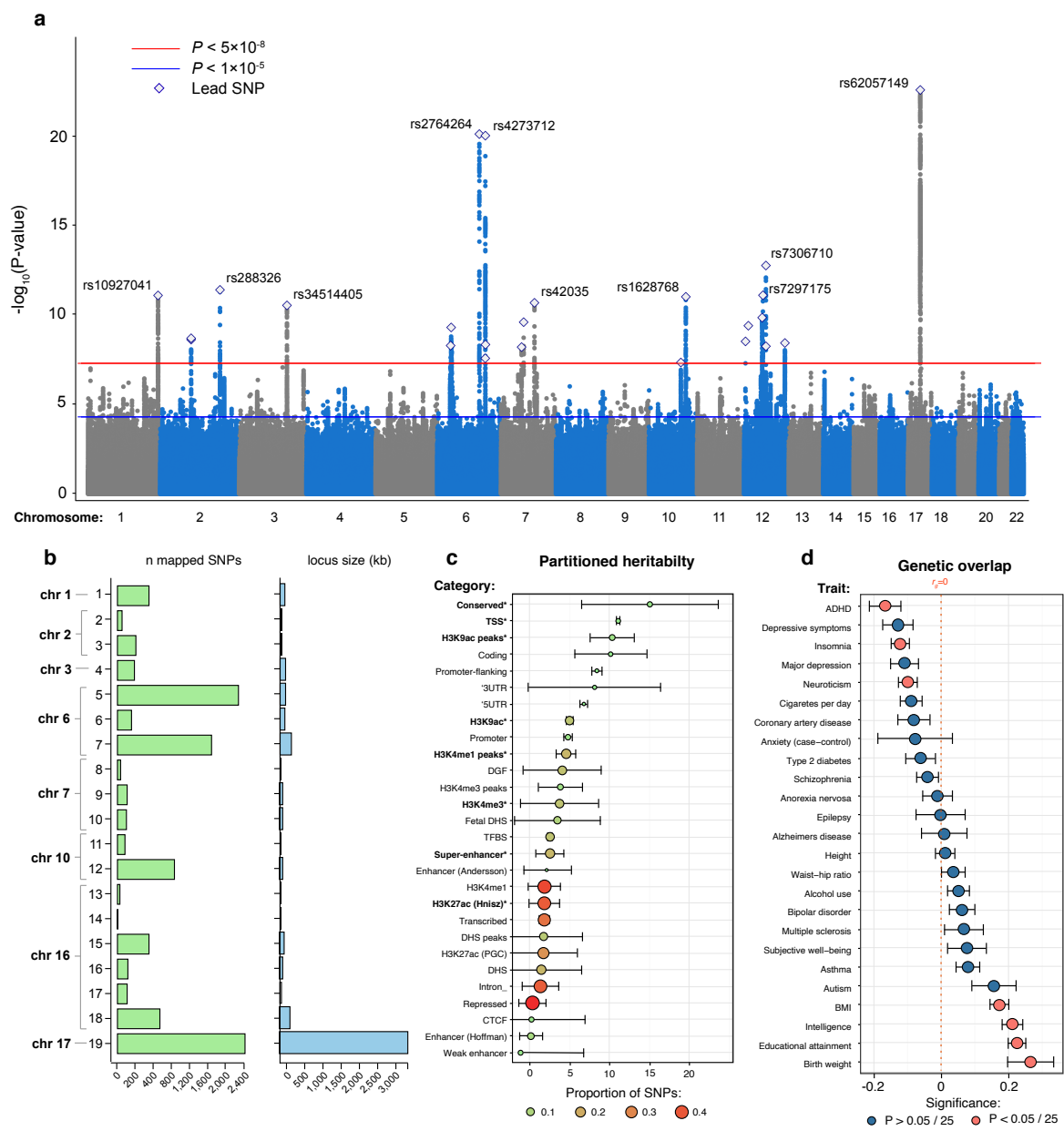

Figure 5.1. Genome-wide meta-analysis and follow-up analyses of brain volume (BV) in UK Biobank, ENIGMA and the GWAS of HC

(a) Manhattan plot of the GWAS meta-analysis of BV in 47,316 individuals showing the genomic position of each SNP on the $x$-axis and the meta-analysis negative $\log _{10}$-transformed $P$ value on the $y$-axis. Independent lead SNPs of each locus are annotated by a diamond. The horizontal red line indicates the genome-wide significance threshold that corresponds to a $P$ value of $5 \times 10^{-8}$, while the horizontal blue indicates the suggestive threshold of $1 \times 10^{-5}$. (b) Overview of the genomic loci sizes and number of SNPs mapped by each locus. (c) Partitioning of the SNP heritability of the BV GWAS meta-analysis by binary SNP annotations using LD Score regression. Enrichment was calculated by dividing the partial heritability of a category by the proportion of SNPs in that category. Significant enrichments, corrected for the number of categories tested $(P<0.05 / 28)$, are highlighted in bold and with an asterisk. (d) Genetic correlations with previous traits estimated using LD Score regression. Red dots denote significant genetic correlation after Bonferroni correction $(P<0.05 / 25)$. 
To test whether specific functional categories of SNPs contribute disproportionally to the heritability of BV, we used LDSC to partition the genetic signal over different SNP annotations (Online Methods). We observed significant heritability enrichment in 8 SNP categories (Fig. 5.1c; Supplementary Table 7), with the strongest enrichment of SNPs in (evolutionary) conserved regions, (enrichment $=15.1, \mathrm{SE}=2.7, P=1.19 \times 10^{-6}$, suggesting a 15 -fold enrichment in $h^{2}$ conveyed by SNPs in these regions compared to the proportion of SNPs in these regions), transcription start sites (TSS; enrichment=11.1, $\mathrm{SE}=3.1, P=1.2 \times 10^{-3}$ ), and H3K9ac peaks (i.e., specific histone modification that is correlated with active promoters; enrichment=10.3, $\mathrm{SE}=3.0 P=1.24 \times 10^{-3}$ ).

\section{BV gene analyses}

To gain insight into which genes may be involved in BV, we mapped the 4,683 candidate SNPs implicated in the BV meta-analysis to genes, using positional mapping, eQTL mapping, and chromatin interaction mapping as implemented in FUMA ${ }^{148}$, and through gene-based association tests as implemented in MAGMA ${ }^{150}$ (Online Methods, Supplementary Fig. 6). In total, 343 unique genes were implicated by at least one of these methods (of these, 321 genes were mapped based on the 18 identified risk loci). Specifically, the 19 risk loci were mapped to 119 genes based on position, 207 genes by eQTL association, 192 genes through chromatin-chromatin interactions (Fig. 5.2a; Supplementary Table 8), and gene-based association testing identified 69 genes (Fig. 5.2b; Supplementary Table 9). Of the 343 genes, 73 genes were replicated from previous studies (38 not replicated), while 270 were novel gene findings (Supplementary Table 10). Overall, 16 genes were implicated by all four methods (FRZB, FOXO3, WBP1L, PRR13, MAP3K12, RAB5B, SUOX, RPS26, ERBB3, PITPNM2, C12orf65, INA, HMGA2, PLEKHM1, MAPT, and KANSL1) of which the first 11 were not identified in previous studies of $\mathrm{BV}^{295,297,301,303,304}$.

Variation in brain volume can be seen as a continuum, with abnormally small or large brains as the extremes. Since monogenic Mendelian disorders are often characterized by abnormal brain development, which frequently results in deviations in brain volume, we performed look-up of the 343 genes implicated in the BV meta-analysis in the Online Mendelian Inheritance in Man database $^{305}$ (OMIM, URLs). We identified 143 monogenic disorders caused by high-penetrance mutations in 89 of the $343 \mathrm{BV}$-implicated genes $(25.9 \%$ vs. $17.1 \%$ in all genes that were tested in the gene-based analysis; $P=2.33 \times 10^{-5}$ ) many of which are commonly associated with abnormal brain development (including microcephalia (CDK6), macrocephalia (PTEN) and megalencephaly (AKT3)), and abnormal growth (tetra-amelia syndrome (WNT3)) (Supplementary Table 11). 
a

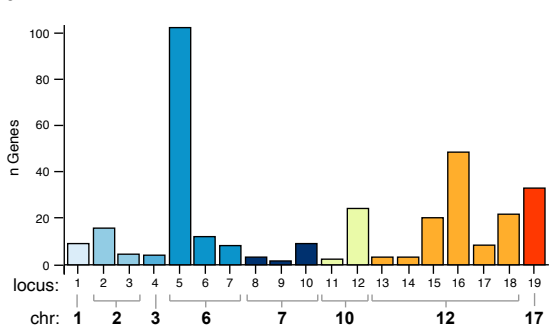

b

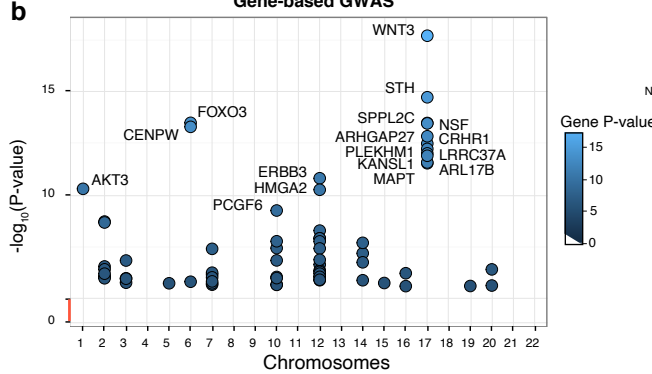

C

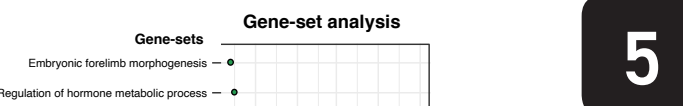

Figure 5.2. Gene analyses based on the GWAS meta-analysis of BV

(a) Number of genes that were mapped by FUMA to each locus. Colors indicate distinct chromosome numbers (b) Significant genes in the gene-based association test in MAGMA after Bonferroni correction $(P<0.05 / 18,169)$. The top 15 most significant genes are annotated with the corresponding gene symbols. (c) Significant MsigDB gene sets after Bonferroni correction $\left(P<3.90 \times 10^{-6}(=0.05 /(12,191+53+565))\right.$ showing the negative $\log _{10}$-transformed $P$ value on the $x$ axis; size and colors of the circles represent the size of the gene set.

To identify functional pathways related to BV, we performed gene-set and gene-expression analysis in MAGMA ${ }^{150}$ (Online Methods). Of the 12,191 tested gene sets (including canonical pathways, gene ontology gene sets, and gene-expression in tissue/cell types), 18 were significantly associated after Bonferroni correction $\left(P_{B O N F}=3.90 \times 10^{-6}(0.05 /(12,191\right.$ gene sets +53 tissue types + 565 cell types)); Fig. 5.2c; Supplementary Table 12-13). Among these significant gene sets were several cell-signaling pathways, including the ErBB3 pathway, the PI3K/AKT signaling in cancer pathway and a pathway related to GRB7 events in ERBB2 signaling. Other pathways were related to (regulation of) developmental processes such as mesenchyme and muscle organ development, or to growth. That is, key cell-signaling pathways were implicated that are involved in normal brain development ${ }^{306}$ and involved in several brain developmental abnormalities ${ }^{307,308}$. Pairwise conditional gene set analysis allows one to disentangle overlapping associations of gene sets (see Online Methods). For instance, to examine whether a gene set is independently associated with a trait, rather than being associated because it is nested within a larger associated gene $\operatorname{set}^{309}$. These analyses indicated that several gene sets (e.g. neuron axogenesis and mesenchyme 
development) constitute independent associations (Supplementary Fig. 7-9, Supplementary Table 14). Conversely, conditional analyses suggest that several signaling pathways (e.g., PI3K events in ERBB2/ERBB4 signaling, GAB1 signalosome) represent a shared underlying signal, since conditional $P$ values were substantially higher than the unconditional $P$ values.

Next we aimed to identify tissue categories and neuronal cell-types that are enriched for gene signal of BV, by linking gene $P$ values to gene-expression in 53 tissue-types ${ }^{310}$ and 565 brain celltypes $^{311}$ (Online Methods). None of the associations of tissue or cell-types passed our stringent multiple testing correction (i.e. correcting for all tested gene sets, tissues and cell types, thus $0.05 / 12,809)$. The strongest evidence of association was observed for three cell-types: TNR-BMP4 polydendrocytes in the subthalamic nucleus, $\left(P=4.67 \times 10^{-3}\right)$, layer 5a cortical $\left(P=8.55 \times 10^{-3}\right)$ and hippocampal endothelial stalk FLT1-LCN2 neurons $(P=0.02)$ (Supplementary Table 12). Polydendrocytes are thought to be precursor cells of oligodendrocytes ${ }^{312}$, a cell-type involved in supporting neuronal health and myelinization of the brain ${ }^{313}$. The endothelial neurons in the hippocampus support formation of the blood-brain barrier, and are involved in regulation of neurogenesis ${ }^{314}$.

\section{Genetic correlations between BV and other traits}

Earlier studies have shown substantial overlap between BV and behavioral traits, reporting phenotypic correlations between BV and e.g. autism ${ }^{315}$, and genetic correlations between BV and intelligence ${ }^{138,316-318}$ and schizophrenia ${ }^{319}$. We used LDSC to estimate the overlap in genetic signal between BV and 25 brain-related and neuropsychiatric traits for which published genome-wide summary statistics based on large samples were available (Online Methods). Significant genetic correlations $(P<0.002 ; 0.05 / 25)$ were observed between $\mathrm{BV}$ and 7 traits, amongst which were positive genetic correlations with educational attainment $\left(r_{g}=0.22, \mathrm{SE}=0.03, P=3.58 \times 10^{-17}\right.$; Fig. 5.1d; Supplementary Table 15) and intelligence $\left(r_{g}=0.21, \mathrm{SE}=0.03, P=9.87 \times 10^{-12}\right)$, and negative genetic correlations with ADHD $\left(r_{g}=-0.17 \mathrm{SE}=0.05, P=3.56 \times 10^{-4}\right)$, neuroticism $\left(r_{g}=-0.10\right.$, $\left.\mathrm{SE}=0.03, P=4.65 \times 10^{-4}\right)$, insomnia $\left(r_{g}=-0.12, \mathrm{SE}=0.03, P=5.76 \times 10^{-6}\right)$, confirming previously reported overlap ${ }^{235,295,320}$.

\section{Genetic overlap with intelligence}

Development of human intelligence has coincided with a strong increase in total size of the brain $^{321}$. Indeed, epidemiological studies have shown overlap in genetic factors between BV and intelligence ${ }^{74,138}$. Here we use GWAS summary statistics of a large recent meta-analysis of intelligence from Savage et al. $(2018)^{74}$, based on data from 14 cohorts $(\mathrm{N}=269,867)$ using different types of intelligence measures, to further explore the nature of the genetic overlap with BV. Except for one, all of these cohorts used intelligence measures that adhere to the idea of a single latent trait $g$ (Spearman's $g$ ) that underlies multiple domains of cognitive ability. Extensive previous research 
has shown that a) correlations between performance on different, validated tests of cognitive ability are high and b) all index the same underlying (statistical) construct, i.e., $g^{292,322}$.

Our analyses confirmed the previously established genetic overlap between BV and intelligence: the estimated $r_{g}$ between BV and intelligence was 0.24 (Supplementary Tables 2, 15), closely resembling the estimates of shared genetic factors between intelligence and gray matter volume $\left(r_{g}=0.29\right)$ and white matter volume $\left(r_{g}=0.24\right)$ obtained from twin studies ${ }^{138}$. To gain insight into the specific genetic factors driving this genetic correlation, we explored the overlap in SNPs, loci and genes associated with both traits.

We first determined whether strongly associated SNPs show the same direction of effect in both traits, by performing a look-up of the lead SNPs of BV in the intelligence GWAS, and vice versa. In line with expectations, we observed a complete sign concordance of the 24 BV lead SNPs in intelligence ( sign concordance $=100 \%, P=1.19 \times 10^{-7}$, Fig. 5.3c) and weaker but still considerable sign concordance of the 243 lead SNPs of intelligence in BV (sign concordance $=62.1 \%$, $P=1.39 \times 10^{-4}$, Fig. 5.3d). Similarly, strong deviations from the expected $P$ value distribution were observed for genes that were significant for BV (69) and intelligence (507) in the gene-based test in MAGMA (Supplementary Fig. 10).

To explore the presence of any directional effects between BV and intelligence, we carried out GWAS summary statistics-based Mendelian Randomization analyses, using the Generalized Summary-data-based Mendelian Randomization package ${ }^{205}$ (GSMR; Online Methods), essentially using independent lead SNPs as instrumental variables (LD: $r^{2}<0.1$ ). GSMR analyses demonstrated a directional effect of $\mathrm{BV}$ on intelligence $\left(b_{x y}=0.154, \mathrm{SE}=0.015, P=1.88 \times 10^{-23}\right.$; Fig. 5.3a) and a less strong yet still highly significant directional effect of intelligence on $\mathrm{BV}\left(b_{x y}=0.139\right.$, $\mathrm{SE}=0.026, P=7.34 \times 10^{-8}$; Fig. 5.3b), suggesting a bidirectional association between these phenotypes, in line with previous reports ${ }^{74}$. We do note, however, that GSMR makes several strong assumptions, such as the absence of a third, mediating, factor, and no horizontal or vertical pleiotropy ${ }^{323}$, which may not always hold. The current results should be interpreted conditional on these assumptions.

To investigate whether specific SNPs or genes could be identified that drive the genetic overlap between intelligence and $\mathrm{BV}$, we performed several cross-trait analyses of SNPs and genes significantly implicated in both traits. We observed physical overlap in 5 out of the 18 genomic loci for BV, and overlap in genes implicated by FUMA ( $\left.\mathrm{n}_{\text {genes }}=81\right)$ and MAGMA ( $\left.\mathrm{n}_{\text {genes }}=24\right)$, resulting in 92 unique overlapping genes (Fig. 5.3e-f; Supplementary Table 16). Conversely, of the 343 genes that were significant for BV, 251 genes were not significantly associated to intelligence (Fig. 5.3e). 

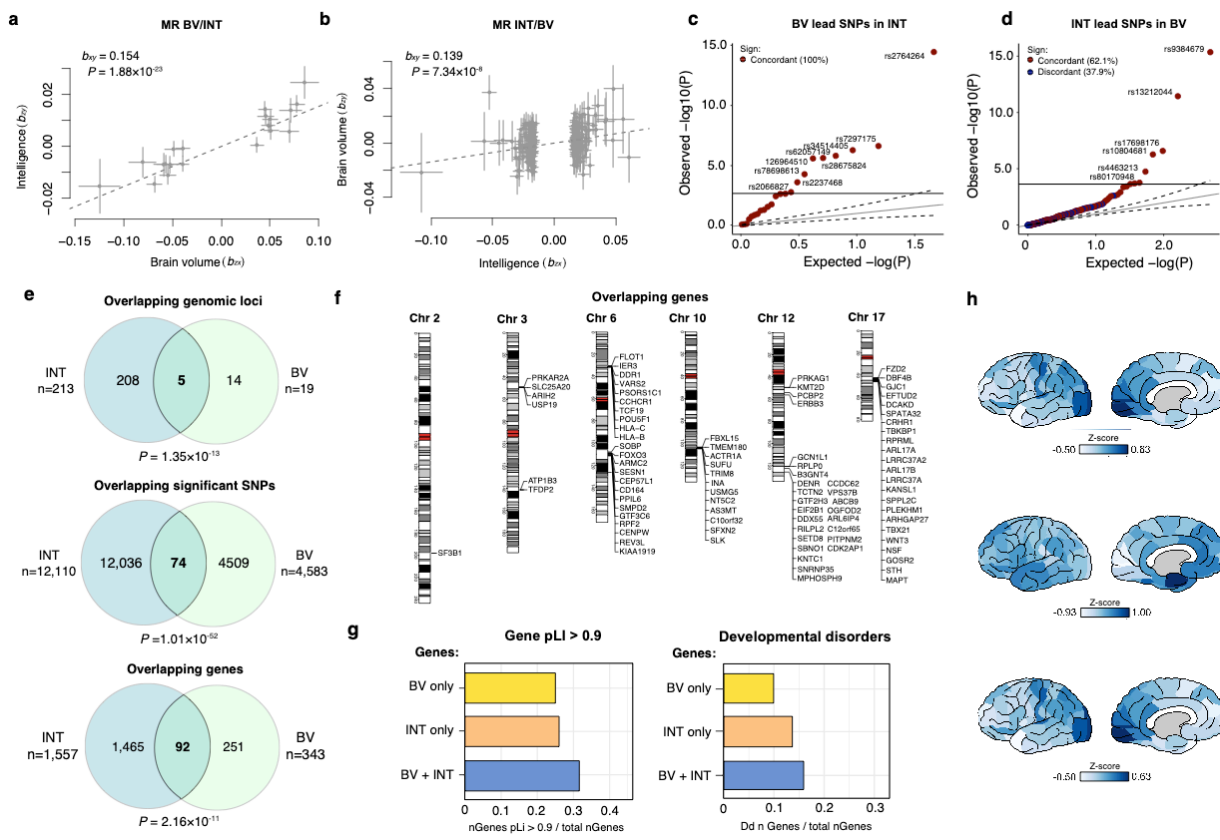

h
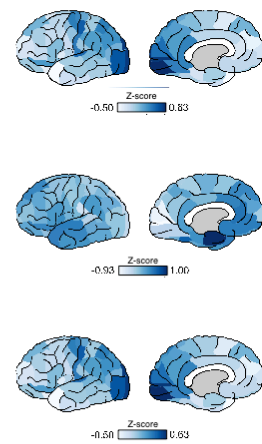

Figure 5.3. Analyses of the genetic overlap between BV and intelligence

(a) Mendelian Randomization analysis, showing the effects and standard errors of each SNP of BV $\left(b_{z y}\right)$ on intelligence $\left(b_{z x}\right)$ and the diagonal line showing the estimated causal effect $\left(b_{x y}\right)$ of BV on intelligence. (b) Similar to a) but of intelligence on BV. (c) Q-Q plot showing the observed and expected $P$ value distribution of 24 lead SNPs of the BV meta-analysis in intelligence and (d) 243 intelligence lead SNPs in BV. The colors denote the concordance (/discordance) of effects between the two traits. (e) Venn diagrams showing the number of overlapping loci, significant SNPs, and genes implicated in BV and intelligence by FUMA and MAGMA. (f) Karyogram showing the genomic position of the 92 genes that were implicated in the GWAS of both BV and intelligence. (g) Comparison of the pLI score (representing tolerance to loss-of-function variants) and fraction of genes involved in developmental disorders (DECIPHER database) between BV-only genes, intelligence-only genes (INT), and genes associated to both traits (BV+INT). (h) Cortical gene expression patterns (darker colors indicate higher rank scores) across 57 cortical areas of the 92 genes that overlap between BV and intelligence (top panel), and the two clusters of genes (middle and bottom panel).

Lookup of gene functions of the 92 overlapping genes in the online GeneCards ${ }^{324}$ repository (URLs) showed strong involvement of these genes in a wide variety of cellular processes and key factors in cell division. When comparing the probability of being loss-of-function (LoF) intolerant ( $\mathrm{pLI}$ score $>0.9)$ between overlapping genes $(n=92)$ and genes observed for only one of these traits $(n=1,926)$, we observe a slightly higher fraction of genes being LoF intolerant in the genes that play a role in both traits $(31.7 \%$ (shared) vs. $26.1 \%$ (intelligence) and $24.5 \%$ (BV), Fig. 5.3g). The 
mutation intolerance of these overlapping genes is further demonstrated by a slightly higher fraction of genes in this category that are associated with monogenic disease (18.3\%) in the OMIM database compared to BV-only (16.8\%) and intelligence-only (17.4\%) genes. Furthermore, we observed a higher proportion of genes that cause monogenic developmental disorders (overlap: 16.0\%, BVonly: $13.7 \%$, intelligence-only: $10.0 \%$ ) in the DECIPHER database of developmental disorders ${ }^{325}$ (Fig. 5.3g).

Previous studies have sought to narrow down the link between intelligence and brain volume to specific subcortical regions, such as the caudate nuclei ${ }^{326}$ or the thalamus ${ }^{327}$. If specific brain regions are related to intelligence, one might expect genes associated to intelligence (or BV) to be differentially expressed across regions. Using data obtained from the Allen Human Brain Atlas $\left(\mathrm{AHBA}^{328}\right.$ ), we examined whether cortical gene expression profiles differed between the 92 genes that overlap between BV and intelligence and the 2,018 genes that associated to BV, intelligence, or both. We compared the gene expression profile of the 92 genes related to both $\mathrm{BV}$ and intelligence to that of genes associated to either or both of the traits $\left(n_{\text {genes }}=2,018\right.$; Fig. 5.3e $)$. Specifically, using permutation analyses we compared the expression of these 92 overlapping genes across 57 brain regions to that of 10,000 randomly selected, equally large, sets of genes drawn from the 2,018 genes related to either or both of the traits. Although we observed overexpression of the 92 overlapping genes in the anterior part of the fusiform $(P=0.0152)$ and the parahippocampal gyrus $(P=0.005)$, none of the associations survived a conservative Bonferroni correction $(P<0.00088$; 0.05/57); Supplementary Table 17). To examine whether clusters of more homogeneously expressed genes exist within the 92 overlapping genes, we additionally performed clustering analysis on the correlations between the expression profiles of genes, aiming to maximize intracluster cohesion, while at the same time maximizing differences between clusters (see Online Methods). Two clusters were identified and only for cluster 2 (representing 42 of the 92 overlapping genes) one of the 57 expression profiles was significantly different from that of random sets, suggesting overexpression in the anterior fusiform gyrus $(P=0.0006$; Supplementary Table 17). Interaction analyses in MAGMA (see Online Methods), testing whether gene expression in specific brain regions contributed significantly to the genetic relationship between BV and intelligence, turned out negative (Supplementary Table 18). Based on these results, we are unable to conclude that the set of overlapping genes is expressed in any particular cortical region.

To prioritize those genes from the 92 overlapping genes, that are more likely to have causal effects on both BV and intelligence, we filtered the set of overlapping genes based on either one of three conditions: 1) the gene contains an ExNS SNP, 2) the gene is part of one of the significant gene sets for BV or intelligence, and 3) the GWAS signal of either trait colocalizes with eQTL signals, using COLOC $^{329}$ (colocalization of GWAS and eQTL signals is compatible with the hypothesis of a common causal variant; see URLs; Supplementary Table 16). Filtering the 92 overlapping genes on the basis of these 3 conditions yielded a selection of 32 potentially functionally interesting genes. For example, we prioritized two genes on chromosome 3 (out of 4 
associated genes in the same region) that may prove particularly interesting candidates for functional follow-up aimed at characterizing the genetic relation between $\mathrm{BV}$ and intelligence: both USP19 and ARIH2 are part of a significantly associated gene set for $\mathrm{BV}$, and in addition USP19 contains an ExNS variant that was significantly associated to intelligence.

To examine whether the gene-set associations are unique to BV and intelligence, respectively, we conducted conditional gene-set analyses (i.e., we conditioned the gene-based signal in BV on the gene-based Z-scores for intelligence, and vice versa). Interestingly, these analyses indicated that the gene sets identified for BV (18) and intelligence (9) are trait specific, since the association remains virtually unchanged when conditioning on the other trait (Supplementary Table 19). Fisher's exact tests showed that the 92 overlapping genes were not significantly enriched in the 27 gene sets associated to BV or intelligence (Supplementary Table 20). However, several genes related to intelligence were included in gene sets observed for $\mathrm{BV}$, including ERBB3 and FOXO3 (see Fig. 5.4), suggesting that, although no single gene set was shared between $B V$ and intelligence, several genes associated with intelligence are located along important signaling pathways implicated in BV.

\subsection{Conclusion}

In conclusion, we studied the genetics of BV and report 14 novel loci and 270 novel genes for BV. In exploring the genetic overlap between BV and intelligence, we identified 92 genes that are associated to both traits, i.e., contributing to the genetic overlap between these traits. Of the 92 overlapping genes, we prioritized 32 genes that might be best candidates for functional follow-up aimed at gaining biological insight into the overlap between BV and intelligence. We did not identify specific biological characteristics or mechanisms that differentiate the 92 overlapping genes from the full set of genes that was associated to BV and/or intelligence, except for an increased intolerance to loss of function mutations. We expect that increasing the sample size, and thus the statistical power, for the BV GWAS will help in further dissecting the shared nature of BV and intelligence. These findings are a first step towards understanding of the genetic architecture of $\mathrm{BV}$, and its shared etiology with intelligence. 


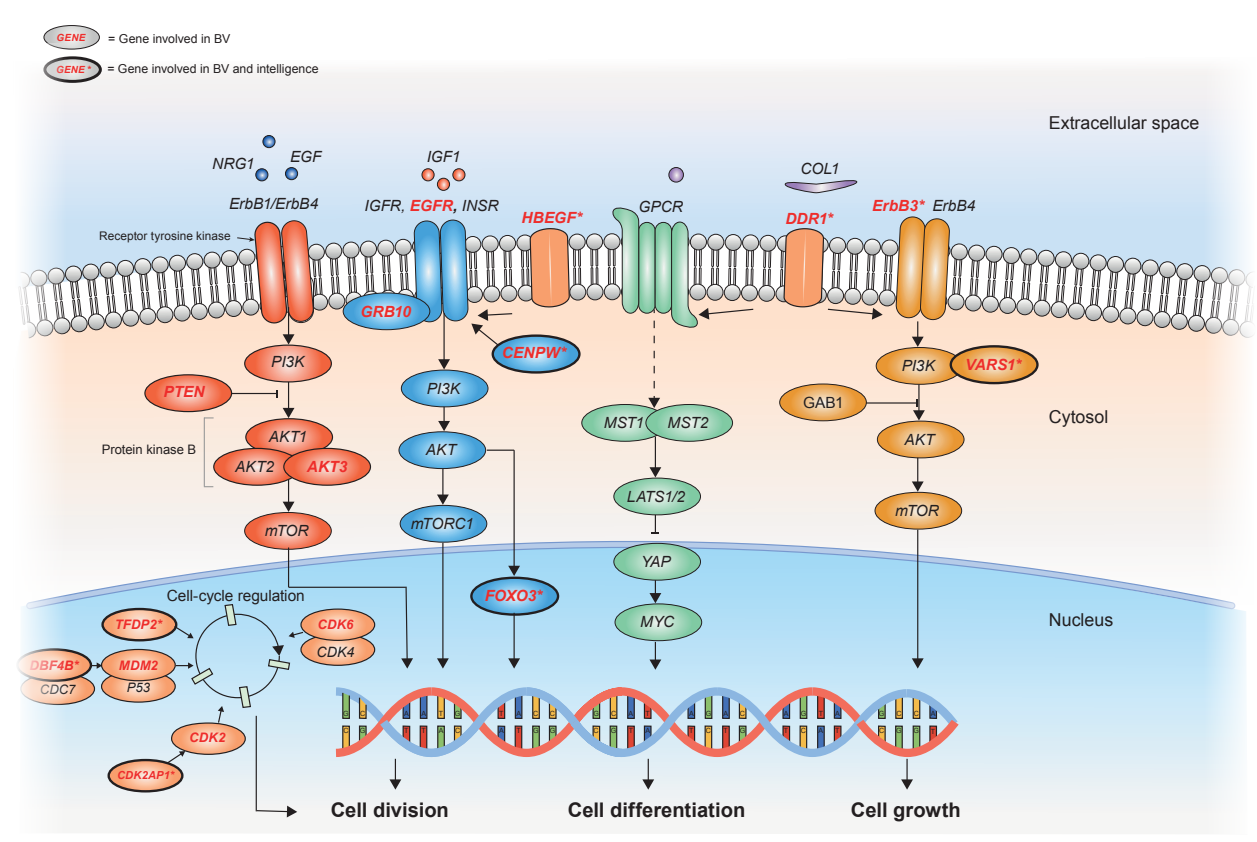

Figure 5.4. Schematic representation of cellular signaling pathways involved in $B V$

Overview of cellular signaling pathways and implicated genes within these pathways that were mapped by the GWAS meta-analysis of BV or overlapped with the genes that were mapped by the GWAS of intelligence. Genes in red font were involved in the meta-analysis of BV, whereas genes in red font in a thick-outlined oval denoted by an asterisk were observed in both the BV and intelligence GWAS. 


\subsection{Methods}

\section{Samples \& phenotypes}

\section{UK Biobank - Brain volume}

The UK Biobank (UKB) constitutes a large data set, combining a wide range of phenotypes with genetic and imaging information. Here, we used processed data of a subset of $\mathrm{N}=21,407$ individuals who underwent a magnetic resonance imaging (MRI) procedure: data were released in the third quarter of 2018. After filtering on quality of the imaging results, relatedness, European ancestry and the availability of relevant covariates (discussed in more detail below), we arrived at a final sample size of $\mathrm{N}=17,062$. The brain volume (BV) phenotype was approximated as follows: $\mathrm{BV}=$ white matter volume + grey matter volume + cerebrospinal fluid volume (UKB field codes 25008 , 25006 and 25004, respectively). The UKB obtained ethical approval from the National Research Ethics Service Committee North West-Haydock (reference 11/NW/0382), and all study procedures were performed in accordance with the World Medical Association for medical research. The current study was conducted under UKB application number 16406.

\section{ENIGMA - Intracranial volume}

We used GWAS summary statistics on intracranial volume (ICV) obtained in a collaboration of cohorts included in the meta-analysis of ICV of the Enhancing NeuroImaging Genetics through Meta-Analysis (ENIGMA) ${ }^{297}$ consortium. ICV was calculated from brain MRI data collected in 50 individual cohorts $(\mathrm{N}=11,373)$. Given the moderate phenotypic and relatively low, but still significant, genetic correlation between brain volume and height (phenotypic $r=0.55$ in UK Biobank; $r_{g}=0.26$ between height in UK Biobank and uncorrected ICV summary statistics from ENIGMA ${ }^{297}$ ) we considered height an important covariate to include in GWAS on volumetric brain measures. Therefore, we used mtCOJO to correct for height, by conditioning on height using UKBderived sumstats (this procedure is described in more detail below). Details on the individual cohorts included in the ENIGMA meta-analysis are described elsewhere ${ }^{297}$. All participants were of European descent and provided informed consent. All studies included in the ENIGMA metaanalysis were approved by their local institutional review board or local ethics committee. Details on genotyping, imputation and analysis procedures can be found in the original publication ${ }^{297}$.

\section{Head circumference GWAS}

In contrast to the two samples discussed above, the GWAS summary statistics obtained from the head circumference (HC) GWAS are based on genetic analysis of an indirect measure of brain volume, i.e., head circumference in children and adults (see Supplementary Methods for more information on the relation between $\mathrm{HC}$ and BV). These summary statistics were generated by meta-analyzing 11 population-based cohorts, resulting in a total sample size of $\mathrm{N}=18,881$. All studies included in the meta-analysis were approved by the local ethics committees, and informed (or parental) consent was obtained from all participants. Data collection, genotyping, imputation 
and analysis procedures were described in detail elsewhere ${ }^{301}$. Since not all individual studies that were included in the HC meta-analysis corrected for height, we first conditioned the HC GWAS summary statistics on height using a procedure similar to that described for the ENIGMA data $\left(r_{g}=0.39\right.$ between height in UK Biobank and uncorrected HC summary statistics from Haworth et al. (2019)).

\section{Genotyping, imputation \& quality control of UKB data}

The genotype data that we used was released by the UKB in March 2018, and concerns an updated version of data released earlier (July 2017). Details on the collection and processing of the genotype data are described elsewhere ${ }^{293}$. To summarize, the UKB genotyped in total 489,212 individuals on two custom made SNP arrays (UK BiLEVE Axiom ${ }^{\mathrm{TM}}$ array covering 807,411 markers $(\mathrm{n}=49,950)$ and UK Biobank ${ }^{\mathrm{TM}}$ Axiom array covering 825,927 markers $(\mathrm{n}=438,427)$, both by Affymetrix). The genotype arrays shared $95 \%$ of marker content. Quality control executed by the UKB team resulted in a total of 488,377 individuals and 805,426 unique markers in the released data. In the version of the data used for the current study (genotype data release of March 2018), genotypes were phased and imputed by the UKB team to a combined reference panel of the Haplotype Reference Consortium and the UK10K. Finally, imputed and quality-controlled genotype data was available for 487,422 individuals and 92,693,895 genetic variants.

Prior to analysis, imputed variants were converted to hard call using a certainty threshold of 0.9. In our own quality control procedure, we excluded SNPs with a low imputation score (INFO score $<0.9)$, low minor allele frequency $(\mathrm{MAF}<0.005)$ and high missingness $(>0.05)$. Furthermore, indels that had the same chromosomal location were excluded. This resulted in a total of 9,203,453 SNPs used for downstream analysis.

To prevent population stratification from biasing the results, we only included individuals from European descent in our analyses. To this end, we projected principal components (PC's) from the 1000 Genomes reference populations ${ }^{174}$ onto the called genotypes available in the UKB data. Participants for whom the projected principal component score was closest (using the Mahalanobis distance) to the average score of the European 1000 Genomes sample ${ }^{213}$ were considered to be of European descent. Participants having a Mahalanobis distance $>6$ S.D. were excluded from further analysis. In an additional quality control step, we excluded participants that 1) had withdrawn their consent, 2) were related to other participants according to the UKB team (i.e., subjects with most inferred relatives, $3^{\text {rd }}$ degree or closer, were removed until no related subjects were present), 3) reported a gender that did not match their genetic gender, or 4) showed sex-chromosome aneuploidy. After filtering availability of imaging data, and MRI scan quality, 17,062 individuals remained for analysis. 


\section{GWAS of brain volume in UKB}

The genome-wide association analyses (GWAS) of BV in the UKB data was conducted in

PLINK ${ }^{175,176}$, using a linear regression model with additive allelic effects. In order to correct for potential subtle population stratification effects, we included 10 genetic PC's as covariates. Genetic PC's were computed using FlashPCA2 ${ }^{177}$ in the QC'ed subset of unrelated European subjects, retaining only independent $\left(r^{2}<0.1\right)$, relatively common (MAF>0.01) and genotyped or very high imputation quality (INFO=1) SNPs ( $n=145,432$ markers). Additional covariates included in the analysis were: age, sex, genotype array, assessment center, standing height (since the genetic correlation between $\mathrm{BV}$ and height in the UKB is $r_{g}=0.26$ ), and the Townsend deprivation index. We refer to the Supplementary Results for a brief comparison of the UKB BV GWAS results where the firsts 10 or the first 100 genetic PC's have been included as covariates.

\section{Conditional GWAS (mtCOJO)}

Multi-trait-based conditional \& joint analysis using GWAS summary data ( $\mathrm{mtCOJO}^{205}$; see URLs) can be used to conduct conditional GWAS analyses. The mtCOJO method is integrated in the GCTA $^{330}$ software, and requires summary-level GWAS data to obtain GWAS results for phenotype A that are conditioned on (i.e., accounted for) phenotype A's genetic relatedness with phenotype B. In the current study, we performed two conditional GWASs, aiming to correct the existing GWAS summary statistics of ICV (ENIGMA) and head circumference (HC-GWAS) for their overlap with the genetic signal of height. First, we ran mtCOJO on the GWAS summary statistics for ICV on the ENIGMA GWAS results, conditioning on UKB-derived summary statistics for standing height. Second, we ran mtCOJO on the GWAS summary statistics of head circumference in the HC GWAS meta-analysis, again conditioning on UKB-derived summary statistics for standing height. The conditioned GWAS results were subsequently included in the GWAS metaanalysis of BV described in the following section.

\section{GWAS meta-analysis of brain volume}

Before carrying out the meta-analysis of brain volume (BV), we performed additional filtering and prepared the summary statistics for each of the individual cohorts. First of all, within the ENIGMA data, SNPs that were available for $N<5,000$ were excluded. We then performed similar filtering on the HC-GWAS data. Secondly, in the UKB data we identified some instances where there were multiple SNPs on the same position, whilst having different alleles. These SNPs were excluded from further analysis. Lastly, we aligned the allele coding of indels in the ENIGMA data to match the coding in the UKB data. A number of indels that was not present in the UKB data was excluded from analysis, since we used the UKB data as reference data for downstream analyses. The summary statistics from the UKB, ENIGMA and the HC-GWAS consortium showed strong genetic correlations ( $r_{g}$ ranging between 0.75 and 1; see Supplementary Table 2), supporting our choice for a meta-analytic approach. 
Using a sample-size weighted $z$-score method in METAL ${ }^{179}$ we combined the GWAS on brain volume in the UKB data $(\mathrm{N}=17,062)$, the GWAS on intracranial volume (conditioned on height) in the ENIGMA results $(\mathrm{N}=11,373)$, and the GWAS on head circumference (conditioned on height) in the HC-GWAS results $(\mathrm{N}=18,881$ ), resulting in a total sample size of $\mathrm{N}=47,316$ (see Supplementary Fig. 1).

\section{Intelligence - GWAS summary statistics}

We used recently published GWAS meta-analysis summary statistics of intelligence ${ }^{74}$ to study the genetic overlap with BV. Data collection procedures and methods are described in detail elsewhere ${ }^{74}$. In comparing the results of BV and intelligence, we updated some of the downstream analyses for intelligence (e.g., we used FUMA version 1.3.5 instead of 1.3.0, we explored a slightly different collection of gene sets, and used GTEx data $\mathrm{v} 7$ instead of v6.1). Therefore, gene-based findings presented in the current study may deviate slightly from those presented in the original study $^{74}$ (Supplementary Table 8). We applied Bonferroni correction for multiple testing to the meta-analytic SNP $P$ values to identify to intelligence associated genetic variants.

\section{Genomic risk loci and functional annotation}

\section{SNP annotation}

We used FUMA ${ }^{148}$ (v1.3.5; see URLs) for the functional annotation of the BV meta-analysis results. FUMA is an online platform that takes GWAS summary statistics as input, and subsequently annotates, prioritizes, and visualizes the results.

Prior to defining genomic risk loci, FUMA identifies variants that are genome-wide significant $\left(5 \times 10^{-8}\right)$, and independent $\left(r^{2}<0.6\right)$ as independent significant SNPs. In the next step, independent significant SNPs that are independent from each other at $r^{2}<0.1$ are denoted lead SNPs. Genotypes from the UKB were used as reference data to infer LD. Finally, FUMA characterizes genomic risk loci by merging LD blocks that are located close to each other ( $<250 \mathrm{~kb}$ apart). Thus, it is possible that one genomic risk locus contains multiple independent significant SNPs or lead SNPs.

In order to obtain information on the functional consequences of SNPs on genes, FUMA performs ANNOVAR ${ }^{214}$ gene-based annotation using Ensembl genes (build 85) for all SNPs in LD $\left(r^{2}>0.6\right)$ with one of the independent significant $S N P S$ and having an association $P$ value lower than $1 \times 10^{-5}$. Additionally, CADD scores ${ }^{197}$, Regulome DB scores ${ }^{181}$, and 15 -core chromatin state ${ }^{182,183}$ are annotated to SNPs by matching chromosome position, reference, and alternative alleles. CADD scores can be used to prioritize genetic variants that are likely to be pathogenic and/or deleterious (CADD scores $>12.37$ suggest a SNP is deleterious). The score is a single measure combining various annotations, and has been shown to correlate with pathogenicity, disease severity, and experimentally measured regulatory effects and complex trait associations ${ }^{215}$. RegulomeDB scores $^{181}$ characterize SNPs by their likelihood to have a regulatory function (with lower scores indicating higher probability of regulatory function). Scores range from 7 , meaning that there is no 
evidence of the variant having a regulatory function, to $1 \mathrm{a}$, meaning that a variant is likely to affect binding and is linked to expression of a gene target ${ }^{181}$. Chromatin state was predicted by ChromHMM $^{182}$ for 127 cell types, using 15 states to classify and describe variants.

\section{Gene mapping}

Using FUMA ${ }^{148}$, all SNPs in genomic risk loci that were genome-wide significant $\left(P<5 \times 10^{-8}\right)$ or were in LD $\left(r^{2}>0.6\right)$ with one of the independent significant SNPS, were mapped to genes. SNPs could be annotated to a gene by either of three strategies. First, positional mapping maps SNPs to protein coding genes based on physical proximity (i.e., within $10 \mathrm{~kb}$ window). Second, eQTL mapping maps SNPs to genes whose expression is associated with allelic variation at the SNP level. Information on eQTLs was derived from 3 publicly available data repositories; GTEx $^{201}$ (v7), the Blood eQTL browser ${ }^{331}$ and the BIOS QTL browser ${ }^{332}$. This strategy maps SNPs to genes up to 1 $\mathrm{Mb}$ apart (cis-eQTLs). We applied a false discovery rate (FDR) of $\leq 0.05$ to limit the results to significant SNP-gene pairs. Third, SNPs were mapped to genes based on significant chromatin interactions between promoter regions of genes (250 bp up- and $500 \mathrm{bp}$ downstream of the transcription start site (TSS)) and a genomic region in a risk locus. In contrast to eQTL mapping, and in the absence of a distance boundary, chromatin interaction mapping may involve long-range interactions. The resolution of chromatin interactions was defined as $40 \mathrm{~kb}$, and hence, interaction regions may comprise multiple genes. In order to prioritize genes implicated by chromatin interaction mapping, information on predicted enhancers and promoters in 111 tissue/cell types from the Roadmap Epigenomics Project ${ }^{67}$ was integrated. We used FUMA to filter on chromatin interactions for which one interaction region overlapped with predicted enhancers, and the other with predicted promoters $250 \mathrm{bp}$ up- and $500 \mathrm{bp}$ downstream of the TSS site of a gene. At the time of writing, FUMA contained Hi-C data of 14 tissue types from the study of Schmitt et al. (2016) ${ }^{217}$. An FDR of $1 \times 10^{-5}$ was used to define significant interactions.

\section{Gene-based analysis}

Genome-wide gene-based analysis (GWGAS) has the potential to identify genes associated to a trait of interest despite the genetic signal of individual SNPs in or nearby the gene not reaching genome-wide significance in SNP-based analyses. In contrast, a gene harboring a few strongly associated SNP and many SNPs that show only very weak association may not be implicated by gene-based analysis. So in addition to gene-mapping in FUMA, we conducted gene-based analysis in MAGMA ${ }^{150}$ to assess the joint effect of all SNPs within all 19,427 protein-coding genes included in the NCBI 37.3 database. MAGMA requires as input the $P$ values derived from SNP-based analyses, in this case the BV meta-analysis results. All SNPs in our BV meta-analysis were annotated to genes, resulting in 18,168 genes that contained at least one SNP in the BV metaanalysis. Besides SNPs located within a gene, we also included SNPs lying within $2 \mathrm{~kb}$ before and $1 \mathrm{~kb}$ after the TSS of the gene. We used Entrez ID as the primary gene ID. MAGMA's gene-based analysis uses a multiple linear principal component regression, where an $F$-test is used to compute 
the gene $P$ value. The model takes linkage disequilibrium between SNPs into account. Genes were considered to be genome-wide significantly associated if the $P$ value survived a Bonferroni correction for multiple testing $\left(0.05 /\right.$ number of genes tested: $\left.P<2.75 \times 10^{-6}\right)$.

\section{Gene set and tissue expression analysis}

Functional gene sets

Gene set analysis was performed using MAGMA ${ }^{150}$, testing 12,191 predefined gene sets in an exploratory fashion. Selected gene sets included canonical pathways $(n=2,199)$ and gene ontology (GO) gene sets $(n=9,996)$. All gene sets were obtained from the Molecular Signatures Database (MSigDB, version 7.0; see URLs). For all gene set analyses, competitive, rather than selfcontained, $P$ values are reported. Competitive gene set analysis tests whether the joint association of genes in a gene set with the phenotype of interest is stronger than that of a randomly selected set of genes of similar size. This approach provides stronger evidence for association of the gene set compared to a self-contained test, where the joint association of genes in a gene set with the phenotype is tested against the null hypothesis of no effect.

\section{Gene-expression analysis}

To assess whether genes associated to our traits of interest are disproportionately expressed in certain tissue- and cell-types, we applied MAGMA's gene-expression analysis to investigate associations with several gene expression profiles. First, we tested tissue gene-expression in 53 different tissue types obtained from the GTEx portal (v.7; see URLs), which include geneexpression data from 13 brain tissue types ${ }^{310}$. Secondly, we tested gene-expression in 565 distinct adult mouse brain cell-types from Dropviz ${ }^{311}$. These data were collected through the use of the Drop-seq technique ${ }^{333}$ by assessing RNA expression in 690,000 individual cells from 9 brain regions of the adult mice brain, which were subsequently grouped to 565 transcriptionally distinct groups of cell-types.

Gene set and gene-expression analyses were Bonferroni corrected for the total number of gene sets, tissue types and single-cell types tested in MAGMA $\left(P<3.90 \times 10^{-6}(=0.05 /(12,191+53+565))\right.$.

\section{Conditional gene set analyses}

In order to gain more insight in the genetic pathways associated to BV and intelligence, we performed conditional gene set analyses using MAGMA ${ }^{150}$. Conditional analyses are conducted to identify MsigDB gene sets that represent independent associations (i.e., in a regression-based framework, we assessed the association between our trait of interest (e.g., BV) and a gene set, conditional on another trait (e.g., intelligence)). Specifically, we determined which gene set associations remain for BV when we condition on intelligence, and vice versa. This approach provides information on whether gene sets are uniquely associated to BV or intelligence, or rather, shared between both traits. For example, if the $P$ value of association between a gene set and BV increases when conditioning on intelligence (i.e., less significant), then this suggests that the gene 
set association is likely shared between both traits, while if the $P$ value between a gene set and BV is unaffected by conditioning on intelligence, then this implies that the association is specific to $\mathrm{BV}$, i.e., not shared with intelligence. Note however that even when the association between a gene set and a phenotype is largely explained by a second, overlapping, gene set, its conditional $P$ value will still be reduced compared to its marginal association $P$ value. Therefore, de Leeuw et al. $(2018)^{309}$ advise to assess the relative degree of association remaining for each gene set. In addition, we conducted pairwise conditional gene set analysis for all 18 gene sets that were significantly associated to BV.

\section{SNP-based heritability and genetic correlations}

We used linkage disequilibrium score regression $(\mathrm{LDSC})^{146}$ to estimate the proportion of phenotypic variance that can be explained by common SNPs, a statistic known as SNP-based heritability, $h^{2}{ }_{S N P}$. We used precomputed LD scores that were calculated using 1000 Genomes European data (see URLs).

Genetic correlations $\left(r_{g}\right)$ between the signal from our BV meta-analysis, intelligence ${ }^{74}$, and 24 psychiatric, behavioral and lifestyle-related traits for which summary-level data were available, were also calculated using $\operatorname{LDSC}^{116,146}$. Genetic correlations for which the $P$ value survived the correction for multiple testing (Bonferroni-corrected $P<0.002(=0.05 / 25)$ ) were considered significant.

\section{Partitioned heritability}

In order to determine whether some functional categories of the genome contribute more than others to the SNP-heritability $\left(h^{2}{ }_{S N P}\right)$ of BV, we performed stratified LDSC ${ }^{198}$. Using this method, we calculated whether any of the 28 specific genomic categories (see URLs) included in the analysis was enriched for SNPs that contribute to $h^{2}$ SNP. Enrichment here is defined as the proportion of $h^{2}$ SNP in a given category divided by the proportion of SNPs in that category (e.g., if enrichment in intronic regions is 4.75 , this indicates that this functional category has a 4.75 -fold higher contribution to $h^{2}$ SNP compared to all tested SNPs).

\section{Mendelian randomization analysis}

Mendelian randomization (MR) analysis was performed using Generalized summary-data-based Mendelian randomization (GSMR ${ }^{205}$; see URLs). The main goal was to examine whether the genetic correlation between $\mathrm{BV}$ and intelligence $\left(r_{g}=0.24\right)$ might be explained by directional effects. Analyses were conducted using forward and reverse GSMR, testing for uni- and bidirectional effects between BV and intelligence. SNPs that showed clear pleiotropic effects on the exposure phenotype and the outcome (i.e., instruments that are associated to both BV as well as intelligence) were excluded from analysis using the HEIDI outlier method. In addition, we 
excluded SNPs that are in LD $\left(r^{2}>0.1\right)$ with one another across both tests (i.e., SNPs that are associated to either BV or intelligence, but are in LD with a second SNP that is used as an instrument for the other trait).

\section{Linking expression of overlapping genes for BV and intelligence to specific brain regions}

To identify brain regions that are associated with genes that are observed in BV and intelligence, we first performed gene-mapping with MAGMA and FUMA based on the summary statistics of the GWAS of each trait separately, after which we extracted the set of genes implicated by any of the gene-mapping strategies in both traits.

Subsequently, we extracted the cortical gene-expression profile for each of the overlapping genes from the Allen Human Brain Atlas ${ }^{328}$ (AHBA), which describes gene-expression data per gene across distinct cortical areas. An expression profile of the set was obtained by taking the average expression across the 92 genes. We also performed clustering on the individual correlation patterns for each of the expression profiles of each of the 92 genes separately, by computing a $92 \times 92$ gene-to-gene correlation matrix, with the level of correlation between two genes taken as the Pearson correlation coefficient between their cortical expression profiles. This matrix was subdivided into clusters (also called modules) using Newman's modularity algorithm ${ }^{334}$, maximizing for each cluster the intra-cluster cohesion and minimizing cohesion between genes of different clusters. Per cluster, a cluster-level expression profile was computed by calculating the average gene expression profile across all genes in the given cluster, resulting in clusters of genes showing distinct brain maps.

\section{Interaction gene-set analyses}

Aiming to determine whether genes associated to both BV and intelligence are over- (or under-) expressed in specific brain regions, we performed interaction analysis as implemented in MAGMA (v1.07b). This analysis tested whether the combined involvement of a gene set (here: genes related to $\mathrm{BV}$ ) and continuous gene properties (here: gene expression) is different from their individual effects on intelligence. A positive interaction effect would suggest that, within the set of BV-related genes, the relation between expression and gene-based Z-scores for intelligence is stronger compared to other genes (i.e., all genes for which both expression data from AHBA, as well as gene-based Z-scores for intelligence were available). This would indicate that gene expression in that specific region plays a particular role in the genetic relation between BV and intelligence.

An interaction analysis was conducted separately for gene expression in each of 57 brain regions defined in the Allen Human Brain Atlas ${ }^{328}$ (AHBA). To control for potential variation in general expression levels across genes, we conditioned on the marginal effects of the BV-related gene set and average gene expression across all 57 regions, as well as for their interaction. The set of BV-related genes consisted of all genes that were significant in MAGMA's gene-based analysis 
and/or mapped from SNP-level results in FUMA. Interaction effects were deemed significant if their $P$ value exceeded the Bonferroni-corrected threshold of $P<8.77 \times 10^{-4}(0.05 / 57)$.

\section{Data availability}

Our policy is to make genome-wide summary statistics (sumstats) publicly available. Summary statistics from our BV meta-analysis are available for download at the website of the department of Complex Trait Genetics, CNCR (see URLs). The GWAS summary statistics from the intelligence meta-analysis conducted by Savage et al. (2018) ${ }^{74}$ are also available from this website.

\section{URLs}

ENIGMA: http://enigma.ini.usc.edu/research/download-enigma-gwas-results/

FUMA GWAS platform: http://fuma.ctglab.nl/

LD Score regression: https://github.com/bulik/ldsc

MAGMA: https://ctg.cncr.nl/software/magma

MsigDB gene sets: http://software.broadinstitute.org/gsea/msigdb/index.jsp

GTEx portal: https://www.gtexportal.org/home/

Mendelian randomization - GSMR: http://cnsgenomics.com/software/gsmr/

Multi-trait-based conditional \& joint analysis using GWAS summary data (mtCOJO):

http://cnsgenomics.com/software/gcta/\#mtCOJO

COLOC R package: https://cran.r-project.org/web/packages/coloc/coloc.pdf

Allen Human Brain Atlas (AHBA): http://human.brain-map.org/

GeneCards: https://www.genecards.org/ 


\subsection{Supplementary methods}

\section{UK Biobank}

We performed a genome-wide association study (GWAS) using data from the UK Biobank Study (UKB, www.ukbiobank.ac.uk). The UKB is a prospective population-based cohort that collects a wide variety of data in over 500,000 participants throughout the $\mathrm{UK}^{35}$. The UKB study received ethical approval from the National Research Ethics Service Committee North West-Haydock (reference 11/NW/0382), and all study procedures were in accordance with the World Medical Association for medical research. The current study was conducted under UKB application number 16406. Between 2006 and 2010, 9.2 million invitations were sent to potential participants between the age of 40 and 69 years who were living within 25 miles distance from one of the 22 UK Biobank research centers, resulting in $\mathrm{N}=503,325$ individuals included at the start of the study. UK Biobank collects high dimensional data, including questionnaire data, anthropometric measurements, DNA collection and multi-organ magnetic resonance imaging (MRI). Our study included MRI imaging data of the brain, which was added to the study protocol from 2015 onwards and second-release imputed genotype data of the full UKB cohort (available since March 2018).

\section{Using head circumference as proxy phenotype for brain volume}

In contrast to the UKB and ENIGMA data, the GWAS summary statistics obtained from the head circumference (HC) GWAS are based on genetic analysis of an indirect measure of brain volume (BV), i.e., $\mathrm{HC}$ in children and adults. Although literature on the relation between $\mathrm{HC}$ and $\mathrm{BV}$ is fairly scarce (and most existing literature focuses on individuals with autism), we here briefly discuss research corroborating the decision to use $\mathrm{HC}$ as proxy for BV. An early study ${ }^{287}$, using autopsy records comprising $\mathrm{HC}$ as well as brain weight measures in the same people, demonstrated a positive relationship between the two traits at least until age 18. A 2002 study $^{335}$ examined the relationship between BV and HC from childhood through adolescence. Subjects were 76 healthy, normal males of 1.7 to 42 years old (most studies that include BV measures have focused on the relationship between $\mathrm{BV}$ or $\mathrm{HC}$ and autism, and since autism is more common in males most subjects are males). The authors reported that $\mathrm{HC}$ was an excellent predictor for $\mathrm{BV}$ in children 6 years of age or younger $(r=0.93)$, however, for the older age groups in their study $\mathrm{HC}$ was only a good predictor of BV if the subjects' age was also considered.

These studies suggest that there is a positive relation between $\mathrm{HC}$ and $\mathrm{BV}$ in the healthy population. Since Haworth et al. (2019), whose GWAS summary statistics we use in our metaanalysis, adjusted the $\mathrm{HC}$ measures for age (within males and females separately) we feel that these $\mathrm{HC}$ summary statistics results can serve as a very good proxy measure for BV. 


\subsection{Supplementary results}

\section{GWAS of brain volume in UK biobank}

After running the GWAS of brain volume (BV) in the UK Biobank data, we inspected the lambda inflation factor as well as the LD Score regression (LDSC) intercept, to check for inflation in the test statistics that is possibly due to insufficient correction for population stratification. A lambda $>1$ suggest an inflation of the genetic effects which can be due to both spurious and genuine effects, and is likely to increase with sample size ${ }^{231}$. An LDSC intercept $>1$ suggests that there is spurious association, and an intercept $<1.10$ is generally considered to suggest that the inflation of the signal is mostly due to genuine association effects. The genomic inflation factor $\lambda_{\mathrm{GC}}$ computed using LDSC, was 1.12 (Supplementary Table 2), suggesting minor inflation in the median test statistic compared to the expected distribution. The LDSC intercept of 1.02, however, suggested that the observed inflation in genetic signal is mostly due to polygenicity and unlikely to be driven by population stratification ${ }^{196}$. Specifically, the LDSC ratio of 0.14 (obtained by dividing the LDSC intercept -1 by the mean $\chi^{2}-1$ ) indicated that as much as $86.0 \%$ of the observed inflation could be ascribed to true polygenicity and large sample size. The UKB GWAS of BV identified 3,610 genome-wide significant (GWS) SNPs $\left(P<5 \times 10^{-8}\right)$ (Supplementary Table 4). FUMA then extracted 9 independent lead SNPs, which were mapped to 9 independent genomic loci harboring 132 genes.

\section{Results of GWAS meta-analysis of BV}

\section{UKB, ENIGMA \& HC-GWAS data}

The main meta-analysis in the current study combined BV GWAS results from the UK Biobank (UKB; N=17,062, ran specifically for the current paper) with ICV GWAS results from the ENIGMA consortium $(\mathrm{N}=11,373)^{297}$ and data on head circumference $(\mathrm{HC})$ from a previous large GWAS meta-analysis $(\mathrm{N}=18,881)^{301}$, resulting in a final meta-analysis of brain volume in $\mathrm{N}=47,316$ individuals (Supplementary Fig. 1). Meta-analysis was run in METAL ${ }^{179}$ using a sample-size weighted $z$-score method.

In order to assess whether there was inflation in the observed test statistics of the meta-analysis compared to expected values, we inspected the Lambda GC $\left(\lambda_{\mathrm{GC}}\right)$ and the LDSC intercept of the meta-analytic results (Supplementary Table 2). A $\lambda_{\mathrm{GC}}>1$ suggests an inflation of the genetic effects, which can be due to both spurious and genuine effects. The value of $\lambda_{\mathrm{GC}}$ is likely to increase with sample size ${ }^{336}$. An LDSC intercept $>1$ suggests that there is spurious association, although an intercept $<1.10$ is usually considered to suggest that the signal is mostly due to genuine association effects $^{146}$. For the current meta-analysis, the $\lambda_{\mathrm{GC}}$ was 1.23 , indicating that, compared to the expected distribution, the median test statistic was inflated. The LDSC intercept of 1.037, however, suggested that the observed inflation is mostly due to genuine polygenicity, rather than caused by population stratification. The BV meta-analysis identified 4,155 genome-wide significant SNPs, 
which FUMA ${ }^{148}$ ascribed to 18 genomic loci. Lead SNPs across all 18 independent genomic risk loci had the same direction of effect in all cohorts in which they were available (Supplementary Table 3), thereby extending our confidence in the results. One additional locus reported in FUMA was deemed unreliable, as it appeared to be driven by a single SNP (12:12871099:G_T or rs2066827; see Supplementary Fig. 4). The SNP of interest had a MAF of 0.23 in the UKB (compared to $\sim 0.36$ in the 1000 Genomes and TOPMED data), and was not in LD with other variants in the $1000 \mathrm{Genomes}$ data.

\section{Functional annotation}

To extract additional biological information from the GWAS results, we ran functional annotation analyses using the FUMA ${ }^{148}$ annotation platform. Annotation was performed on all SNPs that were considered to be a candidate SNP given a) high LD with one of the independent significant SNPs $\left(r^{2}>0.6\right)$ and $\left.b\right)$ a low $P$ value $\left(P<1 \times 10^{-5}\right)$, which resulted in 4,683 candidate SNPs (Supplementary Table 5). We observed that most of these SNPs were located within intergenic regions $(n=751$, $16.0 \%)$ or intronic regions $(\mathrm{n}=2.444,52.2 \%)$, and a small minority of SNPs in exonic regions $(\mathrm{n}=62,1.3 \%$; Supplementary Fig. 5).

Of the 62 SNPs annotated to exonic regions, 18 SNPs were associated with non-synonymous changes in the proteins of the gene (ExNS; Supplementary Table 6). These ExNS SNPs were located in exons of 12 genes, with several genes including multiple ExNS SNPs, e.g., the SPPL2C gene contained 4 ExNS SNPs, all located in exon 1 of this gene. This gene codes for the signal peptide peptidase-like $2 \mathrm{C}$, an intramembrane protease (IMP) that has been shown to play a role in the degradation of signaling peptides in the brain ${ }^{337}$, of which its exact biological substrate remains largely unclear ${ }^{302}$, but which has recently been suggested to protease SNARE proteins ${ }^{338}$. In addition, the MAPT gene contained 3 ExNS SNPs, all of which were located in exon 6. The MAPT gene product is the Microtubule Associated Protein Tau protein, a protein that regulates the assembly of microtubules ${ }^{339}$, and which has been linked to a wide variety of neurodegenerative disorders including Alzheimer's disease ${ }^{340}$, frontotemporal dementia ${ }^{341}$ and Parkinson's disease ${ }^{342}$.

\section{Gene mapping results}

To find genes for BV, we used three strategies to map SNPs to genes in FUMA ${ }^{148}$, as well as genome-wide gene-based association analysis (GWGAS) conducted in MAGMA ${ }^{150}$ (Online Methods; Supplementary Fig. 6). Summary statistics of the meta-analysis of BV (UKB, ENIGMA, HC-GWAS) were used as input for FUMA. FUMA gene-mapping mapped the 18 genomic loci to 119 genes through positional mapping (locus near to, or within a gene), 207 genes through eQTL mapping (locus associated with the expression of a gene), and 192 genes through chromatin-chromatin interactions (locus that physically interacts with a close or distant gene through the 3D structure of the genome), leading to a set of 321 unique genes (Fig 5.2a; Supplementary Table 8). Of these genes, 156 genes (49.1\%) were mapped by more than one genemapping method and 40 genes (12.6\%) by all three mapping methods. Of all 321 genes mapped by 
FUMA, 59 genes had a pLI $>0.90$, suggesting extreme intolerance to loss of function (LoF) mutations (Supplementary Table 8).

In addition, genome-wide gene-based analysis (GWGAS) was carried out in MAGMA ${ }^{150}$. GWGAS identified 69 significant genes (Fig. 5.2b; Supplementary Table 9), of which a large part (11 genes, 15.9\%) were mapped on chromosome 17. Of the 69 significant genes, 44 genes (63.8\%) were also observed in FUMA, and 16 genes (23.2\%) were implicated through all four mapping strategies (GWGAS, positional mapping, eQTL mapping and chromatin-chromatin interaction mapping; FRZB, FOXO3, WBP1L, INA, PRR13, MAP3K12, RAB5B, SUOX, RPS26, ERBB3, HMGA2, PITPNM2, C12orf65, PLEKHM1, MAPT, KANSL1). Of the 16 genes that were observed in all gene-mapping methods, several genes are known to be important in regulatory functions of cell-signaling and cell neuronal development. For instance, the FOXO3 gene is part of the forkhead gene-family that code for transcription factors involved in cell survival. Transcription factors coded by $\mathrm{FOXO} 3$ are phosphorylated by $A K T 1^{343}$ and induce cell death through triggering of apoptosis. Overexpression of this gene has been shown to lead to cell growth decline in cell lines $^{344}$. ERBB3 (also known as HER3: human epidermal growth factor receptor 3) is part of the epidermal growth factor receptor tyrosine kinases and is a receptor for the heregulin protein ${ }^{345}$. Activation of the ErBB3 receptor leads to activation of the PI3K/ATK-signaling pathway which modifies cell differentiation and proliferation ${ }^{346}$.

\section{Gene-set results}

We performed gene-set analysis in MAGMA ${ }^{150}$, using $P$ values of 18,169 genes as input from the prior GWGAS gene-based testing, and tested 12,191 gene-sets extracted from the online molecular signatures database (MsigDB, version 7.0;) repository ${ }^{347}$ for their association with BV. Selected gene sets included expert-curated gene sets and gene ontology (GO) gene sets. We identified 18 significant gene-sets (Supplementary Table 12). Almost all gene-sets showed partial overlap in genes with each other (Supplementary Fig. 7), implying that several significantly associated genes were shared between those gene-sets (Supplementary Fig. 8). Among these genes were ERBB3 $\left(n_{\text {genesets }}=7\right.$, MAGMA gene-based $\left.P=1.54 \times 10^{-11}\right)$, FOXO3 $\left(n_{\text {genesets }}=10\right.$; MAGMA gene-based $\left.P=3.27 \times 10^{-14}\right)$ and $W N T 3\left(n_{\text {genesets }}=11\right.$, MAGMA gene-based $\left.P=205 \times 10^{-18}\right)$. The $E R B B 3$ gene (ErbB2 Receptor Tyrosine Kinase 3) is a receptor tyrosine kinase of the ErbB family that plays an essential role as cell surface receptor for neuregulins. Overexpression of this gene's protein has been reported to be related to various types of cancer. FOXO3, forkhead box O3 (see also Supplementary Results 1.3), is a transcription factor and a key factor in a wide array of cellular functions, including cell proliferation, differentiation and survival ${ }^{348}$. WNT3 (Wnt family member 3 ) is part of a gene family that encodes secreted signaling proteins. Gene expression studies indicate an important role for this gene in some cases of human breast, rectal, lung, and gastric cancer through activation of the WNT-beta-catenin-TCF signaling pathway. The gene has also been linked to Tetra-Amelia Syndrome. These results suggest that similar regulatory biological pathways are involved in normal cell division during brain development as well as in aberrant cell division in the development of cancerous tumors in several types of cancer, which explains why we observed 
significant enrichment of genes in endometrial cancer and PI3K/AKT Signaling in Cancer genesets.

\section{Comparison with GWAS in UKB using 100 genetic principal components}

As per the request of an anonymous reviewer, we repeated the GWAS of BV in the UK Biobank data, using the first 100 genetic principal components (PCs) rather than the first 10 PCs, as we decided to use in the GWAS on the UKB data that was included in our meta-analysis (Supplementary Fig. 11). Genetic PCs were computed on the same data, using the same software and the same procedure as applied previously for computing the first 10 PCs. The LD Score intercept in the 100PC-corrected GWAS (LDSC intercept $=1.020, \mathrm{SE}=0.007)$ remained practically unchanged compared to the 10PC-corrected GWAS (LDSC intercept $=1.019, \mathrm{SE}=0.007$ ). The genetic correlation, as computed using LD Score regression, between both GWAS was approximately $1\left(r_{g}=0.989, \mathrm{SE}=0.002\right)$, alleviating concerns that $10 \mathrm{PCs}$ would be insufficient to correct for subtle population stratification.

Subsequently, we conducted an additional meta-analysis of BV, using the results of the 100PCcorrected GWAS of BV in the UKB rather than our original 10PC-corrected UKB GWAS. The genetic correlation between the original meta-analysis and this second meta-analysis was 0.9979 $(\mathrm{SE}=0.0004)$. Given that we only observe minimal changes in the results when adding up to 100 PCs as covariates in the analyses, we conclude that most of the confounding due to population stratification was corrected for in the 10PC-corrected GWAS in UK Biobank. All the main results are based on the 10 PC-corrected UKB GWAS.

\section{Acknowledgements}

The full GWAS summary statistics from the ENIGMA GWAS meta-analysis were downloaded from http://enigma.ini.usc.edu/research/download-enigma-gwas-results/.

Data on infant head circumference has been contributed by HC-GWAS and was downloaded from https://hdl.handle.net/1839/ff12326d-9688-4a46-bc2a-b50cbbd2b20c.

The Genotype-Tissue Expression (GTEx) Project was supported by the Common Fund of the Office of the Director of the National Institutes of Health, and by NCI, NHGRI, NHLBI, NIDA, NIMH, and NINDS. The data used for the analyses described in this manuscript were obtained from the GTEx Portal (see URLs) on 06/12/2018.

This study makes use of data generated by the DECIPHER community. A full list of centers that contributed to the generation of the data is available from http://decipher.sanger.ac.uk and via email from decipher@sanger.ac.uk. Funding for the project was provided by the Wellcome Trust. 
This work was funded by The Netherlands Organization for Scientific Research (NWO Brain \& Cognition 433-09-228, NWO MagW VIDI 452-12-014, NWO VICI 435-14-005 and 453-07001, 645-000-003). P.R.J. was funded by the Sophia Foundation for Scientific Research (SSWO, grant nr: S14-27).

Analyses were carried out on the Genetic Cluster Computer, which is financed by the Netherlands Scientific Organization (NWO: 480-05-003), by the VU University, Amsterdam, the Netherlands, and by the Dutch Brain Foundation, and is hosted by the Dutch National Computing and Networking Services SurfSARA. This research has been conducted using the UK Biobank Resource (application number 16406). We would like to thank the participants and researchers who collected and contributed to the data. 

6 Discussion 


\subsection{Background}

A little over a decade ago, the advent of genome-wide data gave new impulse to the field of psychiatric genetics, that until then relied mostly on family and twin data. More recently, the collection of data in very large samples $(>100,000)$ has, again, invigorated the study of the genetics underlying psychological and medical complex traits. For some part the increase in sample size, and thus statistical power, is starting to pay off. For instance, a recent study reported that the pedigree heritability of BMI and height could be fully recovered from whole-genome sequence data $^{49}$, implying significant contributions of rare variants. However, for neuropsychiatric traits a substantial part of the phenotypic variance is still not explained by identified genetic variants (i.e., missing or hidden heritability), with depression and anxiety as notorious examples. In my view, the 'success' of GWAS is therefore relative, and efforts to increase $N$ are not proportionate to efforts aimed at addressing other potential reasons for the limitations in gene identification.

In psychiatric genetics, GWAS are generally performed on phenotypic composite scores, such as the (dichotomized) sum of endorsed items or symptoms (e.g., IQ score, MDD case-control status). While such composite scores have proven useful in psychological and psychiatric practice (e.g., in directing therapeutic intervention), using them as target of genetic studies directs the focus of analysis to only those variants that affect the majority of the symptoms; signal of variants affecting only one or a few symptoms is severely diluted. The ubiquitous focus on composite scores in the current literature may be unfortunate as there is no a priori reason to assume that items/symptoms assigned to one and the same disorder are indeed genetically homogeneous.

In this thesis, I presented several studies (Chapters 2-4) aimed at investigating whether traits or disorders are genetically homogeneous, and distinct from one another on a genetic level. In a fourth study (Chapter 5), we dissected the genetic correlation between brain volume and intelligence. The main question addressed by the studies in this thesis is whether the traits that we distinguish on the phenotypic level are also discernible on the genetic level. Identifying those genetic contributions that are unique (e.g., to an individual symptom or trait) as well as shared (e.g. between brain volume and intelligence, or between MDD and anxiety) may provide important leads to understanding the genetic etiology and comorbidity of psychological traits. 


\subsection{Summary of findings}

In Chapters 2 and 3 we focused on neuroticism, a heritable personality trait, and an important risk factor for several psychiatric disorders, such as major depression ${ }^{133}$ and anxiety disorders ${ }^{143,144}$. The main goal of Chapter 2 was to use item-level genetic analyses to assess the genetic homogeneity of neuroticism, i.e., are the individual neuroticism symptoms genetically homogeneous or not? Due to the large sample size, we identified many novel previously unknown genomic regions associated to the overall neuroticism score (i.e., sum-score). In addition, we also found many regions that were associated to one or more of the individual items, but not to the overall sum-score. The genetic correlations among the individual items varied from 0.38 to 0.91 , suggesting that the items are in fact genetically heterogeneous. Subsequently, we were able to establish two clusters of items that were genetically homogeneous, referred to as depressed affect and worry, that were differentially related to external traits. Overall, this study highlights the purpose and relevance of item- or symptom-level analyses as a means to inspect genetic homogeneity. Moreover, it presents a way to construct subsets of genetically similar items or symptoms that can reliably be used in future gene-finding endeavors.

In Chapter 3 we provided a more in-depth investigation of the neuroticism sum-score, but also elaborated on the differences between, and the validity of, the two previously established neuroticism clusters. We identified a number of novel genomic loci, genes, and specific neuronal cell types associated to neuroticism. Additionally, we were able to further specify the genetic multidimensionality of neuroticism. For instance, we identified diverging patterns of genetic correlation to external traits for both clusters. The distinction between the clusters was also demonstrated by the fact that we identified gene-sets and exonic non-synonymous SNPs (SNPs that are highly likely to have functional consequences) that were unique to one of the clusters. Genetic correlations between the additional meta-analysis of depressive symptoms and the neuroticism clusters validated the depressed affect cluster ( $r_{g}=0.84$, compared to $r_{g}=0.60$ for the worry cluster), suggesting that this dimension of neuroticism might be useful for unraveling the neurobiology of not only neuroticism, but also depression.

In Chapter 4 we again employed symptom-level genetic analyses. However, rather than studying the genetic homogeneity within a trait (e.g., neuroticism: Chapter 2), we now addressed the question whether the traditionally defined psychological constructs anxiety, depression and neuroticism are distinguishable on a genetic level. That is, we aimed to test the biological validity of internalizing disorders (i.e., depression, anxiety) and related personality traits (i.e., neuroticism). We found that 34 selected internalizing items could be summarized by four genetically homogeneous clusters. Comparison of GWAS results between the traditional scale sum scores (anxiety, depression, and neuroticism) and the genetic clusters indicated that 1) there are broad genetic influences that are shared among different facets of the internalizing dimension, and 2) there appear to be unique genetic influences that are specific to certain aspects of the internalizing spectrum. Based on these results we conclude that a dual pathway approach, combining increased 
sample size approaches employing so-called "minimal phenotyping" with fine-phenotyping approaches, is likely to increase success in gene identification efforts.

The final research-based chapter in this thesis, Chapter 5, concerned a cross-trait analysis of brain volume and intelligence in which we first present the largest GWAS meta-analysis on human brain volume to date $(\mathrm{N}=54,407)$, and then scrutinized the well-established correlation between these two traits $(r \approx 0.40)$, which has previously been shown to be entirely due to genetic factors ${ }^{138}$. The meta-analysis of brain volume yielded 35 genomic loci (27 novel), implicating 362 genes (of which $24.3 \%$ were implicated in rare monogenic brain disorders) and 23 biological pathways. The genetic correlation between brain volume and intelligence was estimated at 0.23 , and the genetic overlap was partially driven by physical overlap in 5 genomic loci, and 67 genes that are involved in e.g. important signaling pathways and regulation of cell growth. We did not find evidence for specific biological characteristics or mechanisms differentiating these 67 overlapping genes from the genes related to only one of the traits, except for an enriched intolerance to mutations. However, we prioritized 32 of the 67 genes for functional follow-up based on e.g. harboring of exonic nonsynonymous SNPs (ExNS), and colocalization with eQTLs.

In summary, by studying the genetic architecture of individual subscales or items/symptoms, I aimed to determine whether the apparent genetic complexity of neuropsychiatric traits is (at least partly) due to our choice of operationalization. The answer to this question is twofold; on the one hand, our results have clearly shown that the choice of operationalization affects the results of genetic analysis. Studying symptoms provides potentially important information on the extent to which they share the same underlying biology, or which dimensions of different traits are genetically similar. In an ideal scenario this type of analysis might lead to identification of genetically coherent clusters of symptoms within a trait, thereby reducing the genetic complexity. More homogeneous phenotypes are expected to have a more direct relationship to underlying biological pathways, and may therefore provide better understanding of the causal mechanisms. On the other hand, using more detailed phenotypes in genetic analysis is unlikely to, in itself, reduce the genetic complexity to such an extent that clear-cut biological mechanisms are suddenly revealed. Although individual symptoms provide a more specific measure than their sum-scores, symptoms may still be relatively broad (in particular when it concerns psychological traits). Effect sizes of variants identified for individual symptoms are tiny, as they are for sum-scores, requiring very large samples to detect.

Notwithstanding these nuances, the studies presented in this thesis show that genetic investigations into more fine-grained phenotypes and genetic overlap between related traits can provide valuable information. Adopting more detailed phenotyping ("fine phenotyping") will shed more light on unique vs. shared genetic determinants of complex traits, thereby advancing our understanding of psychiatric traits. 


\subsection{Obstacles in the way of studying detailed phenotypes}

As shown in Chapter 2, an item-level approach can provide valuable insights, unique from, and complementing to, analysis of the overall sum-score. However, there are a number of issues that stand in the way of applying such fine-phenotyping approaches on a wider scale. Here I discuss some of these important issues, starting with practical challenges, before venturing into the realm of more philosophical and etiological questions.

\section{The data bottleneck}

Arguably the most obvious hurdle for the symptom-level approaches applied in this thesis (Chapters $2 \& 4$ ), is the absence of detailed phenotype information in large population samples. Generally, a sum-score or case-control status, but no symptom- or item-level data is available. The study of neuroticism items (Chapter 2), the only item-level data for which sufficiently large samples were available, showed the relevance of this type of analysis. We were able to identify genetically homogeneous clusters that 1) validated in an independent dataset and 2) showed differential genetic signal, also adding to previous findings for the overall neuroticism sum score. Given the heterogeneous nature of other neuropsychiatric disorders (e.g. depression, attention deficit/hyperactivity disorder; ADHD), we argue that item-level analysis is likely to be valuable for these traits as well. For instance, it is conceivable that depression-related symptoms like 'concentration problems', 'fatigue' and 'suicidal ideation' have (partially) different genetic determinants. Similarly, previous studies have distinguished between two symptom dimensions of ADHD, that are differentially associated to psychopathology, cognitive control and other external correlates $^{273}$.

Creating a composite score from a set of disparate symptoms, and conducting genetic analysis on that composite score, is likely to introduce substantial noise, complicating the search for associated genes and, more importantly, clear biological pathways. It would be interesting to perform symptom-level analyses across a wide range of psychological traits to determine the extent to which these theoretically distinguished traits are genetically distinct, and specify cross-trait genetic liabilities reported in recent studies ${ }^{349,350}$.

The scarcity of high-quality phenotype data has impeded research on the genetic homogeneity of psychological traits. Individual genetic effects on psychological traits are very small, and therefore, sample sizes need to be very large to be able to detect these modest genetic effects. In order to obtain such sample sizes, data from different sources are often pooled together (e.g., in meta- or mega-analyses). These original studies typically had different aims, were performed on samples from different countries, and routinely make use of different (types of) measurement instruments (e.g., whereas one study may use a questionnaire to assess a trait, another may use a 
diagnostic interview, or a different questionnaire ${ }^{351}$ ). In the absence of a single universal measure for psychological traits, measurements are typically reduced to a composite measure (e.g., sumscore or case-control status). Hence, even if there was information on individual symptoms in some of the samples to begin with, this information is likely to be lost in the process of combining datasets. This has led to the paradox that large samples typically comprise minimal phenotypic information ${ }^{127}$.

Large-scale prospective cohorts like the UK Biobank $\left(\mathrm{UKB}^{35}\right)$ offer more detailed phenotypic information in samples that are sufficiently large to allow genetic association analyses. In Chapter 2 we were able to identify a large number of (novel) genetic variants associated to individual neuroticism items. However, this was only after meta-analyzing two sub samples of the UKB, resulting in $\mathrm{N}>366,000$. When analyses were restricting to subsample 1 , consisting of approximately 110,000 individuals, only a handful of variants reached genome-wide significance, illustrating that samples need to be large indeed, even when studying more detailed phenotypes. Smaller cohorts (e.g. Generation Scotland ${ }^{352,353}$, LifeLines ${ }^{354}$ ) derived from local initiatives are valuable because they typically offer more fine-grained phenotypic measurements, but lack the required power. In short, studying increasingly detailed phenotypes (e.g., symptoms) alone is unlikely to have substantial added value when sample sizes remain small.

Initiatives like the UKB provide some major advantages over combining several smaller cohorts. Firstly, in contrast to meta-analyses, where inclusion criteria often differ across cohorts, samples from the same study are generally relatively homogeneous with respect to e.g. genetic background (i.e., limited genetic variation decreases background noise). Note that at the same time, this relative homogeneity of the study population may hamper generalization of study results (just as the strong sampling bias towards individuals of European descent also complicates generalizability of GWAS results). Secondly, since data collection in such a large-scale enterprise is highly standardized, bias due to differences in measurements, which often complicate metaanalyses, will be reduced. Third, the UKB has been gathering data on additional phenotypes over time, extending, for instance, initial broad category information on anxiety and depression with symptom-level information ${ }^{36}$. This allows for examining potential benefits of fine-phenotyping over studying case-control status, but also e.g. investigation of genetic or symptomatic heterogeneity within cases. The combination of large samples and more specific phenotypes might be an important step towards a better understanding of the genetics and biological mechanisms underlying psychological traits.

\section{Analyzing symptoms is time consuming}

A second factor that might discourage researchers from analyzing individual symptoms rather than traditional composite scores, is the fact that it's relatively time consuming. Running a single GWAS and reporting the main results is considered fairly straightforward nowadays. In contrast, performing rigorous quality control, GWAS and follow-up analyses for numerous separate symptoms is a lot of work. By way of illustration, in Chapter 2 we conducted 27 separate GWAS 
(12 items analyzed separately in two subsets of the data + the neuroticism sum-score and clusters). In case of bivariate analyses, e.g. computing genetic overlap between symptoms, the workload increases even more (e.g., for the 12 neuroticism items we conducted 132 sign concordance tests, which we repeated for several $P$-value thresholds, and we calculated 144 genetic correlations between the 12 items, and 396 genetic correlations with the 33 external traits). In general, summarizing the GWAS results of many phenotypes and summarizing and interpreting the overlap in results between multiple phenotypes is laborious and complex.

In my view, there are three things that might facilitate such multivariate fine-phenotype GWAS studies, given the availability of suitable data. First, and foremost, it is important to create awareness that studying the genetics of detailed phenotypes has the potential of increasing power to detect associated variants. In addition, biological information, and knowledge of biological mechanisms may be gained, which might otherwise be obscured if the phenotype is a mix of disparate, possible biologically different, symptoms. Secondly, we need to work on creating infrastructure to lift the computational barriers. Given the increased number of analyses, symptomlevel analyses can be computationally demanding. Cluster computing facilities, as currently used by many of the labs in the field of psychiatric genetics, need to be used efficiently to reduce computational load. Fortunately, recent efforts by e.g. the Neale Lab (http://www.nealelab.is/ukbiobank/) and the Posthuma lab (Watanabe et al. ${ }^{79}$ ) are already offering (partial) solutions to this problem, providing summary statistics for thousands of traits to be used freely by the entire scientific community. Third, the community would benefit from the implementation of standardized procedures (/pipelines) for analyzing multiple phenotypes and assessing their homogeneity. Facilitating a modus operandi is essential if the goal is to motivate researchers to engage in more elaborate, symptom-level data analysis.

\section{No one solution fits all}

Despite the potential benefits of studying symptoms rather than composite phenotypes, it is very much the question whether it is at all possible to formulate a single approach that benefits genefinding enterprises for all traits. The reasoning behind studying the genetics of symptoms is that lower-level, narrower, phenotypes may have a more direct relationship to the underlying genetics (i.e., whereas a composite score may be the result of a variety of intricately connected biological mechanisms, the neurobiology of a more narrowly defined phenotype might be clearer). In other words, symptoms are more specific than their sum-score, and therefore they may have a closer relationship to the causal genetic variants ${ }^{83}$. A recent study showed that, indeed, genetic variants identified for depression are non-specific, and often shared between depression and other disorders ${ }^{127}$. This is in line with our findings that, on a genetic level, the borders between depression, anxiety and neuroticism are blurred (see Chapter 4).

Symptoms of psychological traits have been defined based on observed patterns of behavior, not based on their genetic distinctness. In this thesis we have studied heterogeneity among such symptoms, purportedly measuring the same trait (e.g., the 12 neuroticism items of the EPQ-RS ${ }^{88}$ ). 
In other words, we studied whether symptoms that are used to measure a single trait are indeed influenced by the same (sets) of genetic variants. However, heterogeneity may also manifest among individuals that endorse the same items. In this scenario individuals that have the same composite score (i.e., the sum of symptoms), and for whom this composite score came about through the same set of endorsed items, may still show different sets of associated genetic variants. In that case, there are multiple genetic routes that lead to the same phenotypic outcome (a concept sometimes referred to as equifinality ${ }^{83}$ ). Hence, even when detailed phenotype data (i.e., symptom-level) are available, is it reasonable to expect to find nicely demarcated underlying genetic pathways or biological mechanisms? Quite possibly there just isn't a one-to-one relationship between whatever psychological trait we choose and the genetic variants responsible. (Note that at the same time, misclassification, or misdiagnosis, of psychiatric traits is common ${ }^{104}$, as are shifts between disorders over time ${ }^{109}$. These phenomena also have a disadvantageous effect on the power to detect associated variants).

In addition, genetic architecture is likely to differ per trait, further limiting the likelihood of establishing a universally optimal approach to be used in gene-finding enterprises. The approach used in Chapter 2, where we were able to establish two genetically homogeneous sets of neuroticism items (worry versus depressed affect), may not necessarily prove effective for other traits. The number of variants influencing a trait (i.e., the degree of polygenicity), the magnitude of these genetic effects (resulting in lower or higher discoverability ${ }^{79}$ ) and the underlying traitgenerating model (i.e., reflective, formative, network, see e.g., Schmittman et al., 2013 ${ }^{91}$ ) all impact the success of such strategies aimed at reducing genetic heterogeneity.

\section{Dynamics of behavior}

Psychological traits are typically measured at one point in time, after which researchers attempt to link genetic variants to this operationalization of the trait in question. This reflects the idea that the state of the trait is stable, non-changing, over the course of time. However, we know reality does not comply with this assumption. Psychiatric disorders may remit, worsen or develop into another disorder ${ }^{109}$. As such, it would likely be more fitting to think of psychological traits as dynamic, rather than stable entities. Yet, the dynamic nature of psychological traits is generally not considered in GWAS. There are examples of studies that distinguish between, for instance, individuals with specific subtypes of bipolar disorder ${ }^{76}$ or that stratify MDD patients by age of onset and illness course ${ }^{355}$. Nonetheless, for the bulk of GWAS research, individuals that once indicated to have experienced a given disorder are treated as cases permanently.

The fact that psychological traits are dynamic in nature, in contrast to other complex traits (e.g., height, which is stable over the course of adult life), could actually aid genetic studies. For instance, distinguishing between individuals suffering from chronic severe depression rather than e.g. a single depressive episode, thereby creating more homogeneous phenotypes, might be a valuable step in future gene-finding enterprises. Likewise, it's conceivable that stratifying bipolar disorder patients by the degree to which they switch between manic and depressed states might reveal 
different genetic pathways. As mentioned earlier for symptom-level analyses, this kind of stratification requires more detailed phenotypic information (in this case, longitudinal measurements on large groups of individuals), which is currently not available.

\subsection{Future directions}

Unraveling the genetics of psychological traits has proven to be a major challenge. Despite the tremendous increases in sample sizes, for many psychiatric disorders and psychological traits, causal mechanisms have yet to be discovered. For instance, although recent large GWAS studies did, for the first time, reveal significantly associated genetic variants for e.g. depression ${ }^{39,40}$, research on polygenicity and discoverability in GWAS studies suggests that samples of millions of individuals may be required to explain a large proportion of the SNP-based heritability for depression ${ }^{79}$. It would be relevant to determine whether symptoms of a traits generally have a lower degree of polygenicity compared to the trait itself (e.g., individual depression symptoms versus a depression case-control measure), and a higher discoverability.

At the same time, the challenges described above have triggered a fast pace of new developments, and the increase in gene discoveries is a cause of optimism. As genetic variants are being identified for many traits, the focus shifts to development of methods aimed at following up on these results. In addition, labs all over the world are realizing a joined force to push the frontiers of genetics, and techniques to improve the quality and quantity of genetic data (and reduce costs of collecting these data) are evolving rapidly.

One example of the interesting advances concerning the genetic data is the collection of whole genome sequencing (WGS) data in large samples. In contrast to the SNP genotype data used in standard GWAS, which covers only a (relatively small) part of the genome, WGS concerns the entire DNA sequence. Hence, WGS obviates the need for imputation (thereby reducing uncertainty induced by imputation strategies), and, importantly, allows for testing effects of rare variants. A recent paper ${ }^{49}$ analyzing WGS data on 21,620 individuals, showed that the heritability of height and BMI could be fully recovered from the $>47$ million genetic variants. Although results may be different for psychological traits (e.g. owing to the noisier phenotypes), the finding that WGS data markedly reduces the missing heritability problem for height and BMI is very promising.

Studies presented in this thesis, as well as accumulating evidence in the literature ${ }^{85,120}$ 122,124,126,127, have prompted the need for collecting more, and more homogeneous, specific, phenotypes. Chapters 2 and 4 emphasized that, even with symptom- or item-level data, sample sizes have to be large in order to detect associated genetic variants. Therefore, large-scale biobank initiatives like the UKB have a key role in collecting this detailed phenotypic information. The prospective nature of projects like the UKB implies that more and more phenotypes, among which are more detailed phenotypes (e.g., symptom-level data for some mental illnesses ${ }^{36}$ ) as well as 
longitudinal measurements, are being added over time. Expanding this information further by collecting high-dimensional phenotypic data ${ }^{356}$ (e.g., symptom-level data on different points in time) would be a relevant and feasible future step.

In light of future data collection endeavors, it would be useful to think about lower-level phenotypes that are specific to a trait of interest, and might have a more coherent underlying biology (and hence, more easily identifiable associated genetic variants). Symptoms of psychological traits are defined based on observable behavior, not based on their biological distinctness. These symptoms typically have clear therapeutic value (e.g., it's useful to know whether a person who suffers from depression has a network of friends or family to rely on, or whether a person considers suicide, in which case direct intervention may be warranted regardless of other symptoms). Despite their therapeutic relevance, such symptoms are not necessarily key to the disorder and as such relevant in the context of gene-finding. Establishing characteristics of a trait that have more direct links to biological mechanisms (i.e., biomarkers) is therefore an important step in the quest for improving our understanding of psychological traits.

The research presented in this thesis has contributed to the body of literature advocating the use of more detailed phenotyping. My hope is that this motivates the future collection and analysis of detailed phenotypes for genetic research into psychological traits. The field of psychiatric genetics is developing rapidly, and exciting times lie ahead. A wealth of genetic data is already at our disposal, linking this to equally rich phenotypic data is guaranteed to advance the field even further. 


\section{References}

1. Merikangas, K. R. et al. Lifetime prevalence of mental disorders in U.S. adolescents: Results from the national comorbidity survey replication-adolescent supplement (NCSA). J. Am. Acad. Child Adolesc. Psychiatry 49, 980-989 (2010).

2. Khan, A. \& Brown, W. A. Antidepressants versus placebo in major depression: An overview. World Psychiatry 14, 294-300 (2015).

3. Kirsch, I. et al. Initial severity and antidepressant benefits: A meta-analysis of data submitted to the food and drug administration. PLoS Med. 5, e45 (2008).

4. Polderman, T. J. C. et al. Meta-analysis of the heritability of human traits based on fifty years of twin studies. Nat. Genet. (2015). doi:10.1038/ng.3285

5. Pettersson, E. et al. Genetic influences on eight psychiatric disorders based on family data of 4,408,646 full and half-siblings, and genetic data of 333,748 cases and controls. Psychol. Med. 49, 351 (2018).

6. Geschwind, D. H. \& Flint, J. Genetics and genomics of psychiatric disease. Science 349, 719-724 (2015).

7. Posthuma, D. et al. Theory and practice in quantitative genetics. Twin Res. 6, 361-376 (2003).

8. Boomsma, D., Busjahn, A. \& Peltonen, L. Classical twin studies and beyond. Nat. Rev. Genet. 3, 872-882 (2002).

9. Turkheimer, E. Three laws of behavior genetics and what they mean. Curr. Dir. Psychol. Sci. 9, 160-164 (2000).

10. International Human Genome Sequencing Consortium. Initial sequencing and analysis of the human genome. Nature 409, (2001).

11. Lander, E. S. Initial impact of the sequencing of the human genome. Nature 470, 187-197 (2011).

12. Frazer, K. a, Murray, S. S., Schork, N. J. \& Topol, E. J. Human genetic variation and its contribution to complex traits. Nat. Rev. Genet. 10, 241-51 (2009).

13. The International HapMap Consortium. A haplotype map of the human genome. Nature 437, 1299-1320 (2005).

14. Price, A. L., Spencer, C. C. A., Donnelly, P., Ave, H. \& Donnelly, P. Progress and promise in understanding the genetic basis of common diseases. (2015).

15. Mills, M. C. \& Rahal, C. A scientometric review of genome-wide association studies. Commun. Biol. 2, (2019).

16. Ioannidis, J. P. A., Ntzani, E. E., Trikalinos, T. A. \& Contopoulos-Ioannidis, D. G. Replication validity of genetic association studies. Nat. Genet. 29, 306-309 (2001).

17. Risch, N. \& Merikangas, K. The future of genetic studies of complex human diseases. Science 273, 1516-1517 (1996).

18. Altmüller, J., Palmer, L. J., Fischer, G., Scherb, H. \& Wjst, M. Genomewide scans of complex human diseases: True linkage is hard to find. Am. J. Hum. Genet. 69, 936-950 (2001).

19. Munafò, M. R., Bowes, L., Clark, T. G. \& Flint, J. Lack of association of the COMT (Val158/108 Met) gene and schizophrenia: a meta-analysis of case-control studies. Mol. Psychiatry 10, 765-770 (2005).

20. Siontis, K. C. M., Patsopoulos, N. A. \& Ioannidis, J. P. A. Replication of past candidate loci for common diseases and phenotypes in 100 genome-wide association studies. Eur. J. Hum. Genet. 18, 832-837 (2010).

21. Zeggini, E. et al. Replication of genome-wide association signals in UK samples reveals risk loci for type 2 diabetes. Science 316, 1336-41 (2007). 
22. Goris, A. et al. No evidence for association with Parkinson disease for 13 singlenucleotide polymorphisms identified by whole-genome association screening. Am. $J$. Hum. Genet. 78, 1088-1090 (1997).

23. Franke, A. et al. Replication of signals from recent studies of Crohn's disease identifies previously unknown disease loci for ulcerative colitis. Nat. Genet. 40, 713-715 (2008).

24. Pe'er, I., Yelensky, R., Altshuler, D. \& Daly, M. J. Estimation of the multiple testing burden for genomewide association studies of nearly all common variants. Genet. Epidemiol. 32, 381-385 (2008).

25. Sham, P. C. \& Purcell, S. M. Statistical power and significance testing in large-scale genetic studies. Nat. Rev. Genet. 15, 335-46 (2014).

26. Kerem, A. B. et al. Identification of the Cystic Fibrosis gene: Genetic analysis. Science 245, 1073-1080 (1989).

27. Cutting, G. R. Cystic fibrosis genetics: From molecular understanding to clinical application. Nat. Rev. Genet. 16, 45-56 (2015).

28. Ong, T. \& Ramsey, B. W. Update in cystic fibrosis 2014. Am. J. Respir. Crit. Care Med. 192, 669-675 (2015).

29. Sullivan, P. F., Kendler, K. S. \& Neale, M. C. Schizophrenia as a complex trait. Arch. Gen. Psychiatry 60, 1187 (2003).

30. Sullivan, P. F., Neale, M. C. \& Kendler, K. S. Genetic Epidemiology of Major Depression: Review and Meta-Analysis. Am. J. Psychiatry 157, 1552-1562 (2000).

31. Franke, B. et al. The genetics of attention deficit/hyperactivity disorder in adults, a review. Mol. Psychiatry 17, 960-987 (2012).

32. Faraone, S. V. et al. Molecular genetics of attention-deficit/hyperactivity disorder. Biol. Psychiatry 57, 1313-1323 (2005).

33. Bouchard, T. J. \& Loehlin, J. C. Genes, evolution, and personality. Behav. Genet. 31, 243 273 (2001).

34. Ripke, S. et al. Genome-wide association analysis identifies 13 new risk loci for schizophrenia. Nat. Publ. Gr. 45, 1150-1159 (2013).

35. Sudlow, C. et al. UK biobank: an open access resource for identifying the causes of a wide range of complex diseases of middle and old age. PLoS Med 12, e1001779 (2015).

36. Davis, K. A. S. et al. Mental health in UK Biobank: development, implementation and results from an online questionnaire completed by 157366 participants. BJPsych Open 4, 83-90 (2018).

37. Major Depressive Disorder Working Group of the Psychiatric GWAS Consortium. A mega-analysis of genome-wide association studies for major depressive disorder. Mol. Psychiatry 18, 497-511 (2013).

38. Demontis, D. et al. Discovery of the first genome-wide significant risk loci for attention deficit/hyperactivity disorder. Nat. Genet. 51, 63-75 (2018).

39. Wray, N. R. et al. Genome-wide association analyses identify 44 risk variants and refine the genetic architecture of major depression. Nat. Genet. 50, 668-681 (2018).

40. Howard, D. M. et al. Genome-wide meta-analysis of depression identifies 102 independent variants and highlights the importance of the prefrontal brain regions. Nat. Neurosci. 22, (2019).

41. Visscher, P. M. et al. 10 Years of GWAS discovery: Biology, function, and translation. Am. J. Hum. Genet. 101, 5-22 (2017).

42. Maher, B. The case of the missing heritability. Nat. News 456, 18-21 (2008).

43. Manolio, T. a et al. Finding the missing heritability of complex diseases. Nature 461, 74753 (2009).

44. Wray, N. R. \& Maier, R. Genetic basis of complex genetic disease: The contribution of disease heterogeneity to missing heritability. Curr. Epidemiol. Reports 1, 220-227 (2014).

45. Marouli, E. et al. Rare and low-frequency coding variants alter human adult height. Nature 542, 186-190 (2017). 
46. Gibson, G. Rare and common variants: Twenty arguments. Nat. Rev. Genet. 13, 135-45 (2012).

47. Kiezun, A. et al. Exome sequencing and the genetic basis of complex traits. Nat. Genet. 44, 623-30 (2012).

48. Zuk, O. et al. Searching for missing heritability: Designing rare variant association studies. PNAS 111, E455-E464 (2014).

49. Wainschtein, P. et al. Recovery of trait heritability from whole genome sequence data. bioRxiv (2019). doi:10.1101/588020

50. McKinney, B. A. \& Pajewski, N. M. Six degrees of epistasis: Statistical network models for GWAS. Front. Genet. 2, 1-6 (2012).

51. Wei, W. H., Hemani, G. \& Haley, C. S. Detecting epistasis in human complex traits. Nat. Rev. Genet. 15, 722-733 (2014).

52. Haworth, C. M. A., Asbury, K., Dale, P. S. \& Plomin, R. Added value measures in education show genetic as well as environmental influence. PLoS One 6, (2011).

53. Boomsma, D. I. et al. An extended twin-pedigree study of neuroticism in the Netherlands Twin Register. Behav. Genet. 48, 1-11 (2018).

54. Hublin, C., Partinen, M., Koskenvuo, M. \& Kaprio, J. Heritability and mortality risk of insomnia-related symptoms: A genetic epidemiologic study in a population-based twin cohort. Sleep 34, 957-964 (2011).

55. Plomin, R. \& Deary, I. J. Genetics and intelligence differences: five special findings. Mol. Psychiatry 20, 98-108 (2015).

56. Deary, I. J., Johnson, W. \& Houlihan, L. M. Genetic foundations of human intelligence. Hum. Genet. 126, 215-232 (2009).

57. Bouchard, T. J. \& McGue, M. Genetic and environmental influences on human psychological differences. J. Neurobiol. 54, 4-45 (2003).

58. Verhulst, B., Neale, M. C., Kendler, K. S. \& Genetics, M. The heritability of alcohol use disorders: a meta-analysis of twin and adoption studies. Psychol. Med. 45, 1061-1072 (2015).

59. de Zeeuw, E. L., van Beijsterveldt, C. E. M., Glasner, T. J., de Geus, E. J. C. \& Boomsma, D. I. Arithmetic, reading and writing performance has a strong genetic component: A study in primary school children. Learn. Individ. Differ. 47, 156-166 (2016).

60. de Zeeuw, E. L., de Geus, E. J. C. \& Boomsma, D. I. Meta-analysis of twin studies highlights the importance of genetic variation in primary school educational achievement. Trends Neurosci. Educ. 4, 69-76 (2015).

61. Wareham, N. J. et al. Variability in the heritability of Body Mass Index: A systematic review and meta-regression. Front. Endocrinol. (Lausanne). 3, 1-16 (2012).

62. Schousboe, K. et al. Sex Differences in Heritability of BMI: A Comparative Study of Results from Twin Studies in Eight Countries. Twin Res. 6, 409-421 (2007).

63. Silventoinen, K. et al. Heritability of adult body height: A comparative study of twin cohorts in eight countries. Twin Res. 6, 399-408 (2007).

64. Mortimer, J. A. et al. Role of genes and environments for explaining Alzheimer disease. Arch. Gen. Psychiatry 63, 168 (2006).

65. Wienke, A., Holm, N. V., Skytthe, A. \& Yashin, A. I. The heritability of mortality due to heart diseases: A correlated frailty model applied to Danish twins. Twin Res. 4, 266-274 (2001).

66. Jang, K. L., Livesley, W. J. \& Vemon, P. A. Heritability of the Big Five personality dimensions and their facets: A twin study. J. Pers. 64, 577-592 (1996).

67. Walters, R. K. et al. Transancestral GWAS of alcohol dependence reveals common genetic underpinnings with psychiatric disorders. Nat. Neurosci. 21, 1656-1669 (2018).

68. Van den Berg, M. et al. Meta-analysis of genome-wide association studies for extraversion: Findings from the Genetics of Personality Consortium. Behav. Genet. (2015). doi:10.1007/s10519-015-9735-5 
69. Nelson, C. P. et al. Association analyses based on false discovery rate implicate new loci for coronary artery disease. Nat. Genet. 49, 1385-1391 (2017).

70. Lee, J. J. et al. Gene discovery and polygenic prediction from a genome-wide association study of educational attainment in 1.1 million individuals. Nat. Genet. 50, 1112-1121 (2018).

71. Nagel, M. et al. Meta-analysis of genome-wide association studies for neuroticism in 449,484 individuals identifies novel genetic loci and pathways. Nat. Genet. 1 (2018).

72. Otowa, T. et al. Meta-analysis of genome-wide association studies of anxiety disorders. Mol. Psychiatry 21, 1391-1399 (2016).

73. Jansen, P. R. et al. Genome-wide analysis of insomnia in 1,331,010 individuals identifies new risk loci and functional pathways. Nat. Genet. 51, 394-403 (2019).

74. Savage, J. E. et al. Genome-wide association meta-analysis in 269,867 individuals identifies new genetic and functional links to intelligence. Nat. Genet. 50, 912-919(2018).

75. Yang, J. et al. Meta-analysis of genome-wide association studies for height and body mass index in 700000 individuals of European ancestry. Hum. Mol. Genet. 27, 3641-3649 (2018).

76. Ruderfer, D. M. et al. Genomic dissection of bipolar disorder and schizophrenia, including 28 subphenotypes. Cell 173, 1705-1715.e16 (2018).

77. Pardiñas, A. F. et al. Common schizophrenia alleles are enriched in mutation-intolerant genes and in regions under strong background selection. Nat. Genet. (2018). doi:10.1038/s41588-018-0059-2

78. Jansen, I. E. et al. Genome-wide meta-analysis identifies new loci and functional pathways influencing Alzheimer's disease risk. Nat. Genet. 51, (2019).

79. Watanabe, K. et al. A global overview of pleiotropy and genetic architecture in complex traits. Nat. Genet. 51, (2019).

80. Chabris, C. F., Lee, J. J., Cesarini, D., Benjamin, D. J. \& Laibson, D. I. The fourth law of behavior genetics. Curr. Dir. Psychol. Sci. 24, 304-312 (2015).

81. Timpson, N. J., Greenwood, C. M. T., Soranzo, N., Lawson, D. J. \& Richards, J. B. Genetic architecture: The shape of the genetic contribution to human traits and disease. Nat. Rev. Genet. 19, 110-124 (2018).

82. Fisher, R. A. The correlation between relatives on the supposition of Mendelian inheritance. Trans. R. Soc. Edinburgh 52, 399-433 (1918).

83. Ormel, J., Hartman, C. A. \& Snieder, H. The genetics of depression: Successful genomewide association studies introduce new challenges. Transl. Psychiatry 9, (2019).

84. Wray, N. R. \& Maier, R. Genetic basis of complex genetic disease: The contribution of disease heterogeneity to missing heritability. Curr. Epidemiol. Reports 1, 220-227 (2014).

85. van der Sluis, S., Verhage, M., Posthuma, D. \& Dolan, C. V. Phenotypic complexity, measurement bias, and poor phenotypic resolution contribute to the missing heritability problem in genetic association studies. PLoS One 5, e13929 (2010).

86. Spitzer, R. L., Kroenke, K., Williams, J. B. W. \& Löwe, B. A brief measure for assessing generalized anxiety disorder. Arch. Intern. Med. 166, 1092 (2006).

87. Beck, A. T., Ward, C. H., Mendelson, M., Mock, J. \& Erbaugh, J. An inventory for measuring depression. Arch. Gen. Psychiatry 4, 561-571 (1961).

88. Eysenck, B. G., Eysenck, H. J. \& Barrett, P. A revised version of the psychoticism scale. Pers. Individ. Dif. 6, 21-29 (1985).

89. Kapur, S., Phillips, A. G. \& Insel, T. R. Why has it taken so long for biological psychiatry to develop clinical tests and what to do about it? Mol. Psychiatry 17, 1174-1179 (2012).

90. Burmeister, M., Mcinnis, M. G. \& Zöllner, S. Psychiatric genetics: Progress amid controversy. Nat. Rev. Genet. 9, 527-540 (2008).

91. Schmittmann, V. D. et al. Deconstructing the construct: A network perspective on psychological phenomena. New Ideas Psychol. 31, 43-53 (2013). 
92. American Psychiatric Association. Diagnostic and statistical manual of mental disorders (5th ed.). (Author, 2013). doi:http://dx.doi.org/10.1176/appi.books.9780890425596

93. Lux, V. \& Kendler, K. S. Deconstructing major depression: a validation study of the DSMIV symptomatic criteria. Psychol. Med. 40, 1679-1690 (2010).

94. Barabási, A.-L., Gulbahce, N. \& Loscalzo, J. Network medicine: A network-based approach to human disease. Nat. Rev. Genet. 12, 56-68 (2011).

95. Borsboom, D., Cramer, A. O. J., Schmittmann, V. D., Epskamp, S. \& Waldorp, L. J. The small world of psychopathology. PLoS One 6, e27407 (2011).

96. Cramer, A. O. J. et al. Dimensions of normal personality as networks in search of equilibrium: You can't like parties if you don't like people. Eur. J. Pers. 26, 414-431 (2012).

97. Cramer, A. O. J. et al. Major depression as a complex dynamic system. PLoS One 11, e0167490 (2016).

98. Borsboom, D., Cramer, O. J. \& Cramer, A. O. J. Network analysis: an integrative approach to the structure of psychopathology. Annu. Rev. Clin. Psychol. 9, 91-121 (2013).

99. Cramer, A. O. J., Waldorp, L. J., van der Maas, H. L. J. \& Borsboom, D. Comorbidity: a network perspective. Behav. Brain Sci. 33, 137-50; discussion 150-93 (2010).

100. Bringmann, L. F., Lemmens, L. H. J. M., Huibers, M. J. H., Borsboom, D. \& Tuerlinckx, F. Revealing the dynamic network structure of the Beck Depression Inventory-II. Psychol. Med. 45, 747-757 (2015).

101. Greene, T., Gelkopf, M., Epskamp, S. \& Fried, E. Dynamic networks of PTSD symptoms during conflict. Psychol. Med. 48, 2409-2417 (2018).

102. Van Borkulo, C. et al. Association of symptom network structure with the course of longitudinal depression. JAMA Psychiatry 72, 1219-1226 (2015).

103. Kendler, K. S. The phenomenology of major depression and the representativeness and nature of DSM criteria. Am. J. Psychiatry 173, 771-780 (2016).

104. Regier, D. a. et al. DSM-5 field trials in the United States and Canada, part II: Test-retest reliability of selected categorical diagnoses. Am. J. Psychiatry 170, 59-70 (2013).

105. Kessler, R. C., Chiu, W. T., Demler, O. \& Walters, E. E. Prevalence, severity, and comorbidity of of 12-month DSM-IV disorders in the National Comorbidity Survey Replication. Arch Gen Psychiatry 62, 617-627 (2005).

106. Klein, D. N. \& Riso, L. P. Psychiatric disorders: Problems of boundaries and comorbidity. in Basic issues in psychopathology. 19-66 (Guilford Press, 1993).

107. Anttila, V. et al. Analysis of shared heritability in common disorders of the brain. Science 360, eaap8757 (2018).

108. Gandal, M. J. et al. Shared molecular neuropathology across major psychiatric disorders parallels polygenic overlap. Science 359, 693-697 (2018).

109. Bromet, E. J. et al. Diagnostic shifts during the decade following first admission for psychosis. Am. J. Psychiatry 168, 1186-1194 (2011).

110. Ruggero, C. et al. Consistency of the diagnosis of major depression with psychosis across 10 years. J. Clin. Psychiatry 72, 1207-1213 (2013).

111. Fried, E. I. \& Nesse, R. M. Depression sum-scores don't add up: why analyzing specific depression symptoms is essential. BMC Med. 13, 72 (2015).

112. Ostergaard, S. D., Jensen, S. O. W. \& Bech, P. The heterogeneity of the depressive syndrome: When numbers get serious. Acta Psychiatr. Scand. 124, 495-496 (2011).

113. Fried, E. I. \& Nesse, R. M. The impact of individual depressive symptoms on impairment of psychosocial functioning. PLoS One 9, e90311 (2014).

114. Fried, E. I., Nesse, R. M., Zivin, K., Guille, C. \& Sen, S. Depression is more than the sum score of its parts: individual DSM symptoms have different risk factors. Psychol. Med. 44, 2067-2076 (2014).

115. Middeldorp, C. M. et al. The genetic association between personality and major depression or bipolar disorder. A polygenic score analysis using genome-wide association 
data. Transl. Psychiatry 1, 1-8 (2011).

116. Bulik-Sullivan, B. K. et al. An atlas of genetic correlations across human diseases and traits. Nat. Genet. 47, 1-9 (2015).

117. Smoller, J. W. et al. Psychiatric genetics and the structure of psychopathology. Mol. Psychiatry (2018). doi:10.1038/s41380-017-0010-4

118. Group, C. \& Consortium, P. G. Genetic relationship between five psychiatric disorders estimated from genome-wide SNPs. 45, (2013).

119. Turkheimer, E. Weak genetic explanation 20 years later: Reply to Plomin et al. (2016). Perspect. Psychol. Sci. 11, 24-28 (2016).

120. Charney, A. W. et al. Evidence for genetic heterogeneity between clinical subtypes of bipolar disorder. Transl. Psychiatry 7, e993 (2017).

121. Laurin, C. A., Hottenga, J., Willemsen, G., Boomsma, D. I. \& Lubke, G. H. Genetic analyses benefit from using less heterogeneous phenotypes: An illustration with the hospital anxiety and depression scale (HADS). Genet. Epidemiol. 39, 317-324 (2015).

122. Milaneschi, Y. et al. Polygenic dissection of major depression clinical heterogeneity. Mol. Psychiatry 1-7 (2015). doi:10.1038/mp.2015.86

123. Kendler, K. S., Aggen, S. H. \& Neale, M. C. Evidence for multiple genetic factors underlying DSM-IV criteria for major depression. JAMA Psychiatry 70, 599-607 (2013).

124. Manchia, M. et al. The impact of phenotypic and genetic heterogeneity on results of genome wide association studies of complex diseases. PLoS One 8, e76295 (2013).

125. Minica, C. C., Boomsma, D. I., Sluis, S. Van Der \& Dolan, C. V. Genetic association in multivariate phenotypic data : Power in five models. Twin Res. Hum. Genet. 13, 525-543 (2010).

126. $\mathrm{Xu}, \mathrm{M} . \mathrm{K}$. et al. Psychometric precision in phenotype definition is a useful step in molecular genetic investigation of psychiatric disorders. Transl. Psychiatry 5, e593 (2015).

127. Cai, N., Kendler, K. S. \& Flint, J. Minimal phenotyping yields GWAS hits of low specificity for major depression. bioRxiv (2018).

128. Berg, M. Van Den et al. Harmonization of Neuroticism and Extraversion phenotypes across inventories and cohorts in the Genetics of Personality Consortium : an application of Item Response Theory. Behav. Genet. 44, 295-313 (2014).

129. Moor, M. H. M. De et al. Meta-analysis of genome-wide association studies for personality. Mol. Psychiatry 17, 337-349 (2012).

130. Vinkhuyzen, A. A. E. et al. Common SNPs explain some of the variation in the personality dimensions of neuroticism and extraversion. Transl. Psychiatry 2, e102 (2012).

131. Smith, D. J. et al. Genome-wide analysis of over 106000 individuals identifies 9 neuroticism-associated loci. Mol. Psychiatry 21, 1-9 (2016).

132. Lo, M. et al. Genome-wide analyses for personality traits identify six genomic loci and show correlations with psychiatric disorders. Nat. Genet. (2016). doi:10.1038/ng.3736

133. Kendler, K. S. \& Myers, J. The genetic and environmental relationship between major depression and the five-factor model of personality. Psychol. Med. 40, 801-806 (2010).

134. Kotov, R., Gamez, W., Schmidt, F. \& Watson, D. Linking “ big ” personality traits to anxiety, depressive , and substance use disorders: A meta-analysis. Psychol. Bull. 136, 768-821 (2010).

135. Kendler KS, Neale MC, Kessler RC, Heath AC \& Eaves LJ. Major depression and generalized anxiety disorder: Same genes, (partly) different environments? Arch. Gen. Psychiatry 49, 716-722 (1992).

136. Kendler, K. S. et al. The structure of genetic and environmental risk factors for syndromal common DSM-IV. Am. J. Psychiatry 168, 29-39 (2011).

137. Hettema, J. M., Prescott, C. A., Myers, J. M., Neale, M. C. \& Kendler, K. S. The structure of genetic and environmental risk factors for anxiety disorders in men and women. Arch. Gen. Psychiatry 62, 182 (2005). 
138. Posthuma, D. et al. The association between brain volume and intelligence is of genetic origin. Nat. Neurosci. 5, 83 (2002).

139. Hyde, C. L. et al. Identification of 15 genetic loci associated with risk of major depression in individuals of European descent. Nat. Genet. 48, 1031-1036 (2016).

140. Sniekers, S. et al. Genome-wide association meta-analysis of 78,308 individuals identifies new loci and genes influencing human intelligence. Nat. Genet. (2017). doi:10.1038/ng.3869

141. Okbay, A. et al. Genetic variants associated with subjective well-being, depressive symptoms, and neuroticism identified through genome-wide analyses. Nat. Genet. 48, 624-636 (2016).

142. Hawi, Z. et al. The molecular genetic architecture of attention deficit hyperactivity disorder. Mol. Psychiatry 20, 289-297 (2015).

143. Middeldorp, C. M. et al. The association of personality with anxious and depressive psychopathology. in Biology of Personality and Individual Differences 251-272 (2006).

144. Hettema, J. M., Neale, M. C., Myers, J. M., Prescott, C. A. \& Kendler, K. S. A populationbased twin study of the relationship between neuroticism and internalizing disorders. Am. J. Psychiatry 163, 857-864 (2006).

145. Kendler, K. S., Gatz, M., Gardner, C. O., Pedersen, N. L. \& Virginia, V. Personality and major depression: A Swedish longitudinal, population-based twin study. Arch. Gen. Psychiatry 63, 1113-1120 (2006).

146. Bulik-Sullivan, B. K. et al. LD Score regression distinguishes confounding from polygenicity in genome-wide association studies. Nat. Genet. 47, 291-295 (2015).

147. Tian, C. et al. Analysis and application of European genetic substructure using $300 \mathrm{~K}$ SNP information. PLoS Genet. 4, (2008).

148. Watanabe, K., Taskesen, E., Bochoven, A. van \& Posthuma, D. Functional mapping and annotation of genetic associations with FUMA. Nat. Commun. 8, (2017).

149. Genetics of Personality Consortium. Meta-analysis of genome-wide association studies for neuroticism, and the polygenic association with major depressive disorder. JAMA Psychiatry 72, 642-650 (2015).

150. de Leeuw, C. A., Mooij, J. M., Heskes, T. \& Posthuma, D. MAGMA: Generalized geneset analysis of GWAS data. PLoS Comput. Biol. 11, 1-19 (2015).

151. Subramanian, A. et al. Gene set enrichment analysis: A knowledge-based approach for interpreting genome-wide expression profiles. Proc. Natl. Acad. Sci. 102, 15545-15550 (2005).

152. GTEx Consortium. The genotype-tissue expression (GTEx) pilot analysis: multitissue gene regulation in humans. Science 348, 648-660 (2015).

153. Astle, W. J. et al. The allelic landscape of human blood cell trait variation and links to common complex disease. Cell 167, 1415-1429.e19 (2016).

154. Do, C. B. et al. Web-based genome-wide association study identifies two novel loci and a substantial genetic component for parkinson's disease. PLoS Genet. 7, (2011).

155. Nalls, M. a et al. Large-scale meta-analysis of genome-wide association data identifies six new risk loci for Parkinson's disease. Nat. Genet. 056, 1-7 (2014).

156. Jun, G. et al. A novel Alzheimer disease locus located near the gene encoding tau protein. Mol. Psychiatry 21, 108-117 (2016).

157. Li, Q. S., Tian, C., Seabrook, G. R., Drevets, W. C. \& Narayan, V. A. Analysis of 23andMe antidepressant efficacy survey data: implication of circadian rhythm and neuroplasticity in bupropion response. Transl. Psychiatry 6, e889 (2016).

158. Graff, M. et al. Genome-wide physical activity interactions in adiposity - A metaanalysis of 200,452 adults. PLoS Genet. 13, 1-26 (2017).

159. Justice, A. E. et al. Genome-wide meta-analysis of 241,258 adults accounting for smoking behaviour identifies novel loci for obesity traits. Nat. Commun. 8, 14977 (2017).

160. de Lange, K. M. et al. Genome-wide association study implicates immune activation of 
multiple integrin genes in inflammatory bowel disease. Nat. Genet. 49, 256-261 (2017).

161. Suhre, K. et al. Connecting genetic risk to disease end points through the human blood plasma proteome. Nat. Commun. 8, 14357 (2017).

162. Okbay, A. et al. Genome-wide association study identifies 74 loci associated with educational attainment. Nature 533, 539-542 (2016).

163. The Autism Spectrum Disorders Working Group of The Psychiatric Genomics Consortium. Meta-analysis of GWAS of over 16,000 individuals with autism spectrum disorder highlights a novel locus at 10q24.32 and a significant overlap with schizophrenia. Mol. Autism 8, 21 (2017).

164. Ripke, S. et al. Biological insights from 108 schizophrenia-associated genetic loci. Nature 511, (2014).

165. Nagel, M. et al. GWAS Meta-analysis of neuroticism $(\mathrm{N}=449,484)$ identifies novel genetic loci and pathways. bioRxiv (2017).

166. Mor, N. et al. Evaluating the invariance of the factor structure of the EPQ-R-N among adolescents. J. Pers. Assess. 90, 66-75 (2008).

167. Wechsler, D. Adult intelligence scale. New York 21, (1997).

168. Wechsler, D. Wechsler adult intelligence scale-Fourth Edition (WAIS-IV). San Antonio, TX NCS Pearson 22, 498 (2008).

169. Petrill, S. A., Saudino, K. S., Wilkerson, B. \& Plomin, R. Genetic and environmental molarity and modularity of cognitive functioning in 2-year-old twins. Intelligence $\mathbf{2 9}, 31-$ 43 (2001).

170. Price, T. S. et al. Genetic and environmental covariation between verbal and nonverbal cognitive development in infancy. Child Dev. 71, 948-959 (2000).

171. Posthuma, D., De Geus, E. J. C. \& Boomsma, D. I. Perceptual speed and IQ are associated through common genetic factors. Behav. Genet. 31, 593-602 (2001).

172. Rijsdijk, F. V., Vernon, P. A. \& Boomsma, D. I. Application of hierarchical genetic models to raven and WAIS subtests: A Dutch twin study. Behav. Genet. 32, 199-210 (2002).

173. Plomin, R. \& Spinath, F. Genetics and general cognitive ability. Nature 402, C25-C29 (1999).

174. Auton, A. et al. A global reference for human genetic variation. Nature 526, 68-74 (2015).

175. Chang, C. C. et al. Second-generation PLINK: rising to the challenge of larger and richer datasets. Gigascience 4, 1 (2015).

176. Purcell, S. et al. PLINK: a tool set for whole-genome association and population-based linkage analyses. Am. J. Hum. Genet. 81, 559-575 (2007).

177. Abraham, G. \& Inouye, M. Fast principal component analysis of large-scale genome-wide data. PLoS One 9, 1-5 (2014).

178. McCarthy, M. I. M. I. et al. Genome-wide association studies for complex traits: consensus, uncertainty and challenges. Nat. Rev. Genet. 9, 356-69 (2008).

179. Willer, C. J., Li, Y., Abecasis, G. R. \& Overall, P. METAL: fast and efficient metaanalysis of genomewide association scans. Bioinformatics 26, 2190-2191 (2010).

180. Franke, B. et al. Genetic influences on schizophrenia and subcortical brain volumes: largescale proof-of-concept and roadmap for future studies. Nat. Neurosci. 19, 420-431 (2016).

181. Boyle, A. P. et al. Annotation of functional variation in personal genomes using RegulomeDB. Genome Res. 22, 1790-1797 (2012).

182. Ernst, J. \& Kellis, M. ChromHMM: automating chromatin-state discovery and characterization. Nat. Methods 9, 215-216 (2012).

183. Roadmap Epigenomics Consortium et al. Integrative analysis of 111 reference human epigenomes. Nature 518, (2015).

184. Schmitt, A. D. et al. A compendium of chromatin contact maps reveals spatially active regions in the human genome. Cell Rep. 17, 2042-2059 (2016).

185. Kendler, K. S. \& Myers, J. The genetic and environmental relationship between major 
depression and the five-factor model of personality. Psychol. Med. 40, 801-806 (2010).

186. Middeldorp, C. M. et al. The association of personality with anxious and depressive psychopathology. Biol. Personal. Individ. Differ. 251-272 (2006).

187. Hayes, J. F. et al. Association of late adolescent personality with risk for subsequent serious mental illness among men in a Swedish nationwide cohort study. JAMA Psychiatry 54, 948-963 (2017).

188. Smeland, O. B. et al. Identification of genetic loci shared between schizophrenia and the Big Five personality traits. Sci. Rep. 7, 1-9 (2017).

189. Van Os, J. \& Jones, P. B. Neuroticism as a risk factor for schizophrenia. Psychol. Med. 31, 1129-1134 (2001).

190. Terracciano, A. et al. Genome-wide association scan for five major dimensions of personality. Mol. Psychiatry 15, 647-656 (2010).

191. Lo, M. et al. Genome-wide analyses for personality traits identify six genomic loci and show correlations with psychiatric disorders. Nat. Genet. 49, 152-156 (2016).

192. Nagel, M., Watanabe, K., Stringer, S., Posthuma, D. \& Van der Sluis, S. Item-level analyses reveal genetic heterogeneity in neuroticism. Nat. Commun. 9, 905 (2018).

193. Bycroft, C. et al. Genome-wide genetic data on $\sim 500,000$ UK Biobank participants. bioRxiv (2017).

194. Eriksson, N. et al. Web-based, participant-driven studies yield novel genetic associations for common traits. PLoS Genet. 6, 1-20 (2010).

195. Skol, A. D., Scott, L. J., Abecasis, G. R. \& Boehnke, M. Joint analysis is more efficient than replication-based analysis for two-stage genome-wide association studies. Nat. Genet. 38, 209 (2006).

196. Yang, J. et al. Genomic inflation factors under polygenic inheritance. Eur. J. Hum. Genet. 19, 807-812 (2011).

197. Kircher, M. et al. A general framework for estimating the relative pathogenicity of human genetic variants. Nat. Genet. 46, 310-315 (2014).

198. Finucane, H. K. et al. Partitioning heritability by functional annotation using genome-wide association summary statistics. Nat. Publ. Gr. 47, 1228-1235 (2015).

199. Euesden, J., Lewis, C. M. \& O'Reilly, P. F. PRSice: Polygenic Risk Score software. Bioinformatics 31, 1466-1468 (2015).

200. Yang, J. et al. Modeling linkage disequilibrium increases accuracy of polygenic risk scores. Am. J. Hum. Genet. 97, 576-592 (2015).

201. The GTEx Consortium. The Genotype-Tissue Expression (GTEx) pilot analysis: Multitissue gene regulation in humans. Science 348, 648-660 (2015).

202. Skene, N. G. et al. Genetic identification of brain cell types underlying schizophrenia. Nat. Genet. (2018). doi:10.1038/s41588-018-0129-5

203. Bycroft, C. et al. Genome-wide genetic data on $~ 500,000$ UK Biobank participants. bioRxiv (2017). doi:http://dx.doi.org/10.1101/166298

204. Bulik-Sullivan, B. et al. LD score regression distinguishes confounding from polygenicity in genome-wide association studies. Nat. Genet. 47, 291-295 (2015).

205. Zhu, Z. et al. Causal associations between risk factors and common diseases inferred from GWAS summary data. Nat. Commun. 9, 224 (2018).

206. Griffith, M. et al. DGIdb: Mining the druggable genome. Nat. Methods 10, 1209-1210 (2013).

207. Cotto, K. C. et al. DGIdb 3.0: a redesign and expansion of the drug-gene interaction database. Nucleic Acids Res. gkx1143-gkx1143 (2017).

208. Luciano, M. et al. Association analysis in over 329,000 individuals identifies 116 independent variants influencing neuroticism. Nat. Genet. 50, 6-11 (2018).

209. John, O. P. \& Srivastava, S. The Big Five trait taxonomy: history, measurement, and theoretical perspectives. Handb. Personal. Theory Res. 2, 102-138 (1999).

210. Soto, C. J. \& John, O. P. Ten facet scales for the Big Five Inventory: Convergence with 
NEO PI-R facets, self-peer agreement, and discriminant validity. J. Res. Pers. 43, 84-90 (2009).

211. Costa, P. \& McCrae. Professional Manual: Revised NEO Personality Inventory (NEO-PI$R$ ) and NEO Five-Factor-Inventory (NEO-FFI). (Psychological Assessment Resources: Odessa, FL, 1992).

212. Auton, A. et al. A global reference for human genetic variation. Nature 526, 68-74 (2015).

213. Webb, B. T. et al. Molecular genetic influences on normative and problematic alcohol use in a population-based sample of college students. Front. Genet. 8, 1-11 (2017).

214. Wang, K., Li, M. \& Hakonarson, H. ANNOVAR: functional annotation of genetic variants from high-throughput sequencing data. Nucleic Acids Res. 38, e164-e164 (2010).

215. Kircher, M. et al. A general framework for estimating the relative pathogenicity of human genetic variants. Nat. Genet. 46, 310-315 (2014).

216. Watanabe, K., Taskesen, E., Bochoven, A. van \& Posthuma, D. Functional mapping and annotation of genetic associations with FUMA. Nat. Commun. 8, 1826 (2017).

217. Schmitt, A. D. et al. A compendium of chromatin contact maps reveals spatially active regions in the human genome. Cell Rep. 17, 2042-2059 (2016).

218. Roadmap Epigenomics Consortium et al. Integrative analysis of 111 reference human epigenomes. Nature 518, 317-330 (2015).

219. Croft, D. et al. The Reactome pathway knowledgebase. Nucleic Acids Res. 42, D472D477 (2014).

220. Coleman, J. et al. Functional consequences of genetic loci associated with intelligence in a meta-analysis of 87,740 individuals. bioRxiv (2017).

221. Skene, N. G. et al. Genetic identification of brain cell types underlying schizophrenia. bioRxiv (2017).

222. Zhu, Z. et al. Causal associations between risk factors and common diseases inferred from GWAS summary data. Nat. Commun. 9, 224 (2018).

223. Finucane, H. K. et al. Partitioning heritability by functional annotation using genome-wide association summary statistics. Nat. Genet. 47, 1228-1235 (2015).

224. Griffith, M. et al. DGIdb: mining the druggable genome. Nat. Methods 10, 1209-1210 (2013).

225. Cotto, K. C. et al. DGIdb 3.0: a redesign and expansion of the drug-gene interaction database. Nucleic Acids Res. (2017). doi:10.1093/nar/gkx1143

226. Vilhjálmsson, B. J. et al. Modeling linkage disequilibrium increases accuracy of polygenic risk scores. Am. J. Hum. Genet. 97, 576-592 (2015).

227. Euesden, J., Lewis, C. M. \& O'Reilly, P. F. PRSice: polygenic risk score software. Bioinformatics 31, 1466-1468 (2015).

228. Nagel, M., Watanabe, K., Stringer, S., Posthuma, D. \& van der Sluis, S. Item-level analyses reveal genetic heterogeneity in neuroticism. Nat. Commun. 9, 905 (2018).

229. Howard, D. M. et al. Genome-wide association study of depression phenotypes in UK Biobank $(n=322,580)$ identifies the enrichment of variants in excitatory synaptic pathways. bioRxiv 168732 (2017).

230. Wray, N. R. \& Sullivan, P. F. Genome-wide association analyses identify 44 risk variants and refine the genetic architecture of major depression. bioRxiv 167577 (2017).

231. Yang, J. et al. Genomic inflation factors under polygenic inheritance. 807-812 (2011). doi:10.1038/ejhg.2011.39

232. Locke, A. E. et al. Genetic studies of body mass index yield new insights for obesity biology. Nature 518, 197 (2015).

233. Kemp, J. P. et al. Identification of 153 new loci associated with heel bone mineral density and functional involvement of GPC6 in osteoporosis. Nat. Genet. 49, 1468-1475 (2017).

234. Ripke, S. et al. Biological insights from 108 schizophrenia-associated genetic loci. Nature 511, 421 (2014).

235. Okbay, A. et al. Genome-wide association study identifies 74 loci associated with 
educational attainment. Nature 533, 539 (2016).

236. Freedman, M. L. et al. Assessing the impact of population stratification on genetic association studies. Nat. Genet. 36, 388 (2004).

237. Flint, J. The genetic basis of neuroticism. Neurosci. Biobehav. Rev. 28, 307-316 (2004).

238. Nestler, E. J. \& Hyman, S. E. Animal models of neuropsychiatric disorders. Nat. Neurosci. 13, 1161 (2010).

239. Seong, E., Seasholtz, A. F. \& Burmeister, M. Mouse models for psychiatric disorders. TRENDS Genet. 18, 643-650 (2002).

240. Lampis, V., Maziade, M. \& Battaglia, M. Animal models of human anxiety disorders: reappraisal from a developmental psychopathology vantage point. Pediatr Res 69, 77R84R (2011).

241. Ménard, C., Hodes, G. E. \& Russo, S. J. Pathogenesis of depression: insights from human and rodent studies. Neuroscience 321, 138-162 (2016).

242. de Leeuw, C. A., Mooij, J. M., Heskes, T. \& Posthuma, D. MAGMA: generalized geneset analysis of GWAS data. PLoS Comput. Biol. 11, 1-19 (2015).

243. Gale, C. R. et al. Pleiotropy between neuroticism and physical and mental health : findings from 108038 men and women in UK Biobank. Transl. Psychiatry 6, e791-7 (2016).

244. Martin, P. et al. Personality and longevity: findings from the Georgia Centenarian Study. Age (Omaha). 28, 343-352 (2006).

245. Sniekers, S. et al. Genome-wide association meta-analysis of 78,308 individuals identifies new loci and genes influencing human intelligence. Nat. Genet. 49, 1107 (2017).

246. Lahey, B. B. Public health significance of neuroticism. American Psychologist 64, 241256 (2009).

247. Panula, P., Sundvik, M. \& Karlstedt, K. Developmental roles of brain histamine. Trends Neurosci. 37, 159-168 (2014).

248. Noble, E. P. D2 dopamine receptor gene in psychiatric and neurologic disorders and its phenotypes. Am. J. Med. Genet. Part B Neuropsychiatr. Genet. 116, 103-125 (2003).

249. Nelson, M. R. et al. The support of human genetic evidence for approved drug indications. Nat. Genet. 47, 856 (2015).

250. Boyle, E. A., Li, Y. I. \& Pritchard, J. K. An expanded view of complex traits: from polygenic to omnigenic. Cell 169, 1177-1186 (2017).

251. Luciano, M. et al. 116 independent genetic variants influence the neuroticism personality trait in over 329,000 UK Biobank individuals. Nat. Genet. 50, (2017).

252. Cai, N. et al. Sparse whole-genome sequencing identifies two loci for major depressive disorder. Nature 523, 588 (2015).

253. Fried, E. I. \& Nesse, R. M. Depression is not a consistent syndrome: An investigation of unique symptom patterns in the STAR*D study. J. Affect. Disord. 172, 96-102 (2015).

254. Clark, S. L., Gillespie, N. A., Adkins, D. E., Kendler, K. S. \& Neale, M. C. Psychometric modeling of abuse and dependence symptoms across six illicit substances indicates novel dimensions of misuse. Addict. Behav. 53, 132-140 (2015).

255. Beseler, C. et al. Is there heterogeneity among syndromes of substance use disorder for illicit drugs? Addict. Behav. 31, 929-947 (2006).

256. Prescott, C. A., Kendler, K. S., Neale, M. C., Aggen, S. H. \& Crabbe, J. Evidence for multiple genetic factors underlying the DSM-IV criteria for alcohol dependence. Mol. Psychiatry 17, 1306-1315 (2011).

257. Edwards, A. C. et al. Meta-analysis of positive and negative symptoms reveals schizophrenia modifier genes. Schizophr. Bull. 42, 279-287 (2016).

258. Krueger, R. F. \& Markon, K. E. Reinterpreting Comorbidity: A Model-Based Approach to Understanding and Classifying Psychopathology. Annu. Rev. Clin. Psychol. 2, 111-133 (2006).

259. Roy, M.-A., Neale, M. C., Pedersen, N. L., Mathé, A. A. \& Kendler, K. S. A twin study of generalized anxiety disorder and major depression. Psychol. Med. 25, 1037 (1995). 
260. Kendler, K. S., Gardner, C. O., Gatz, M. \& Pedersen, N. L. The sources of co-morbidity between major depression and generalized anxiety disorder in a Swedish national twin sample. Psychol. Med. 37, 453-462 (2007).

261. Kendler, K. S. Major depression and generalised anxiety disorder: Same genes, (partly) different environments - Revisited. J. Lifelong Learn. Psychiatry II, 416-425 (2004).

262. Subbarao, A. et al. Common genetic and environmental influences on major depressive disorder and conduct disorder. J. Abnorm. Child Psychol. 36, 433-444 (2008).

263. Lahey, B. B., Krueger, R. F., Rathouz, P. J., Waldman, I. D. \& Zald, D. H. A hierarchical causal taxonomy of psychopathology across the life span. Psychol. Bull. 143, 142-186 (2017).

264. Selzam, S. \& Coleman, J. R. I. A polygenic p factor for major psychiatric disorders. (2018). doi:10.1038/s41398-018-0217-4

265. Allegrini, A. G. et al. The p factor: Genetic analyses support a general dimension of psychopathology in childhood and adolescence. bioRxiv 591354 (2019). doi: $10.1101 / 591354$

266. Kendler, K. S., Zachar, P. \& Craver, C. What kinds of things are psychiatric disorders? Psychol. Med. 41, 1143-1150 (2011).

267. Fried, E. I. Problematic assumptions have slowed down depression research: why symptoms, not syndromes are the way forward. Front. Psychol. 6, 1-11 (2015).

268. Watanabe, K. et al. A global view of pleiotropy and genetic architecture in complex traits. bioRxiv 500090 (2018).

269. Bycroft, C. et al. Genome-wide genetic data on $\sim 500,000$ UK Biobank participants. (2017). doi:http://dx.doi.org/10.1101/166298

270. Kroenke, K., Spitzer, R. L., Williams, J. B. W. \& Löwe, B. The Patient Health Questionnaire Somatic, Anxiety, and Depressive Symptom Scales: A systematic review. Gen. Hosp. Psychiatry 32, 345-359 (2010).

271. Purves, K. L. et al. The common genetic architecture of anxiety disorders. bioRxiv 203844 (2017). doi:10.1101/203844

272. Nagel, M. et al. Meta-analysis of genome-wide association studies for neuroticism in 449,484 individuals identifies novel genetic loci and pathways. Nat. Genet. 50, 920-927 (2018).

273. Martel, M. M., Roberts, B., Gremillion, M., Eye, A. Von \& Nigg, J. T. External Validation of Bifactor Model of ADHD: Explaining Heterogeneity in Psychiatric Comorbidity, Cognitive Control, and Personality Trait Profiles Within DSM-IV ADHD. J. Abnorm. Child Psychol. 39, 1111-1123 (2011).

274. American Psychiatric Association. Diagnostic and Statistical Manual of Mental Disorders DSM-IV-TR (Text Revision). (American Psychiatric Association, 2000).

275. Spitzer, R. L., Kroenke, K., Williams, J. B. W. \& Patient Health Questionnaire Primary Care Study Group. Validation and Utility of a Self-report Version of PRIME-MD. JAMA 282, 1737-1744 (1999).

276. Spitzer, R. L. et al. Utility of a New Procedure for Diagnosing Mental Disorders in Primary Care. JAMA 272, 1749 (1994).

277. Kessler, R. C. \& Ustün, T. B. The World Health Organization Composite International Diagnostic Interview. WHO World Ment. Heal. Surv. Glob. Perspect. Epidemiol. Ment. Disord. 7, 58-90 (2008).

278. Walter, K. et al. The UK10K project identifies rare variants in health and disease. Nature 526, 82-89 (2015).

279. McCarthy, S. et al. A reference panel of 64,976 haplotypes for genotype imputation. Nat. Genet. 48, 1279-1283 (2016).

280. Webb, B. T. et al. Molecular Genetic Influences on Normative and Problematic Alcohol Use in a Population-Based Sample of College Students. Front. Genet. 8, 1-11 (2017).

281. Grotzinger, A. D. et al. Genomic SEM provides insights into the multivariate genetic 
architecture of complex traits. bioRxiv (2019).

282. Abraham, G., Qiu, Y. \& Inouye, M. FlashPCA2: principal component analysis of Biobank-scale genotype datasets. Bioinformatics 33, 2776-2778 (2017).

283. Hogan, M. J. et al. Cerebellar brain volume accounts for variance in cognitive performance in older adults. Cortex 47, 441-450 (2011).

284. MacLullich, A. M. J. et al. Intracranial capacity and brain volumes are associated with cognition in healthy elderly men. Neurology 59, 169-174 (2002).

285. Ritchie, S. J. et al. Beyond a bigger brain: Multivariable structural brain imaging and intelligence. Intelligence 51, 47-56 (2015).

286. Lee, J. J., McGue, M., Iacono, W. G., Michael, A. M. \& Chabris, C. F. The causal influence of brain size on human intelligence: Evidence from within-family phenotypic associations and GWAS modeling. Intelligence 75, 48-58 (2019).

287. Epstein, H. T. \& Epstein, E. B. The Relationship between Brain Weight and Head Circumference from Birth to Age 18 Years. Am. J. Phys. Anthr. 48, 471-474 (1978).

288. Vernon, P. A., Wickett, J. C., Bazana, P. G. \& Stelmack, R. M. The neuropsychology and psychophysiology of human intelligence. in Handbook of Intelligence 245-264 (Cambridge University Press, 2000). doi:https://doi.org/10.1017/CBO9780511807947.013

289. McDaniel, M. A. Big-brained people are smarter: A meta-analysis of the relationship between in vivo brain volume and intelligence. Intelligence 33, 337-346 (2005).

290. Pietschnig, J., Penke, L., Wicherts, J. M., Zeiler, M. \& Voracek, M. Meta-analysis of associations between human brain volume and intelligence differences: How strong are they and what do they mean? Neurosci. Biobehav. Rev. 57, 411-432 (2015).

291. Nave, G., Jung, W. H., Karlsson Linnér, R., Kable, J. W. \& Koellinger, P. D. Are Bigger Brains Smarter? Evidence From a Large-Scale Preregistered Study. Psychol. Sci. 30, 4354 (2019).

292. Deary, I. J., Penke, L. \& Johnson, W. The neuroscience of human intelligence differences. Nat. Rev. Neurosci. 11, 201-211 (2010).

293. Bycroft, C. et al. The UK Biobank resource with deep phenotyping and genomic data. Nature 562, 203-209 (2018).

294. Dekkers, I. A., Jansen, P. R. \& Lamb, H. J. Obesity, brain volume, and white matter microstructure at MRI: A cross-sectional UK biobank study. Radiology 291, 763-771 (2019).

295. Adams, H. H. H. H. et al. Novel genetic loci underlying human intracranial volume identified through genome-wide association. Nat. Neurosci. 19, 1569-1582 (2016).

296. Taal, H. R. et al. Common variants at $12 \mathrm{q} 15$ and $12 \mathrm{q} 24$ are associated with infant head circumference. Nat. Genet. 44, 532 (2012).

297. Hibar, D. P. et al. Common genetic variants influence human subcortical brain structures. Nature 520, 224-229 (2015).

298. Hshieh, T. T. et al. Head circumference as a useful surrogate for intracranial volume in older adults. Int Psychogeriatr. 28, 157-162 (2017).

299. Martini, M., Klausing, A., Lüchters, G., Heim, N. \& Messing-Jünger, M. Head circumference - a useful single parameter for skull volume development in cranial growth analysis? Head Face Med. 14, 1-8 (2018).

300. Wolf, H., Kruggel, F., Hensel, A., Wahlund, L. \& Arendt, T. The relationship between head size and intracranial volume in elderly subjects. Brain Res. 973, 74-80 (2003).

301. Haworth, S. et al. Low-frequency variation in TP53 has large effects on head circumference and intracranial volume. Nat. Commun. 10, (2019).

302. Müller, S. A., Scilabra, S. D. \& Lichtenthaler, S. F. Proteomic substrate identification for membrane proteases in the brain. Front. Mol. Neurosci. 9, 96 (2016).

303. Ikram, M. A. et al. Common variants at $6 \mathrm{q} 22$ and $17 \mathrm{q} 21$ are associated with intracranial volume. Nat. Genet. 44, 539-544 (2012). 
304. Rob Taal, H. et al. Common variants at $12 \mathrm{q} 15$ and $12 \mathrm{q} 24$ are associated with infant head circumference. Nat. Genet. 44, 532-538 (2012).

305. McKusick, V. A. Mendelian Inheritance in Man and its online version, OMIM. Am. J. Hum. Genet. 80, 588-604 (2007).

306. Tschopp, O. et al. Essential role of protein kinase $\mathrm{B} \gamma(\mathrm{PKB} \gamma / \mathrm{Akt} 3)$ in postnatal brain development but not in glucose homeostasis. Development 132, 2943-2954 (2005).

307. Lee, J. H. et al. De novo somatic mutations in components of the PI3K-AKT3-mTOR pathway cause hemimegalencephaly. Nat. Genet. 44, 941 (2012).

308. Poduri, A. et al. Somatic activation of AKT3 causes hemispheric developmental brain malformations. Neuron 74, 41-48 (2012).

309. de Leeuw, C. A., Stringer, S., Dekkers, I. A., Heskes, T. \& Posthuma, D. Conditional and interaction gene-set analysis reveals novel functional pathways for blood pressure. Nat. Commun. 9, (2018).

310. Lonsdale, J. et al. The Genotype-Tissue Expression (GTEx) project. Nature Genetics 45, 580-585 (2013).

311. Saunders, A. et al. Molecular diversity and specializations among the cells of the adult mouse brain. Cell 174, 1015-1030 (2018).

312. Nishiyama, A., Komitova, M., Suzuki, R. \& Zhu, X. Polydendrocytes (NG2 cells): multifunctional cells with lineage plasticity. Nat. Rev. Neurosci. 10, 9 (2009).

313. Pfeiffer, S. E., Warrington, A. E. \& Bansal, R. The oligodendrocyte and its many cellular processes. Trends Cell Biol. 3, 191-197 (1993).

314. Wilhelm, I., Nyúl-Tóth, Á., Suciu, M., Hermenean, A. \& Krizbai, I. A. Heterogeneity of the blood-brain barrier. Tissue Barriers 4, (2016).

315. Sacco, R., Gabriele, S. \& Persico, A. M. Head circumference and brain size in autism spectrum disorder: A systematic review and meta-analysis. Psychiatry Res. Neuroimaging 234, 239-251 (2015).

316. van der Schot, A. C. et al. Influence of genes and environment on brain volumes in twin pairs concordant and discordant for bipolar disorder. Arch. Gen. Psychiatry 66, 142-151 (2009).

317. Posthuma, D. et al. Genetic correlations between brain volumes and the WAIS-III dimensions of verbal comprehension, working memory, perceptual organization, and processing speed. Twin Res. Hum. Genet. 6, 131-139 (2003).

318. Posthuma, D. et al. Multivariate genetic analysis of brain structure in an extended twin design. Behav. Genet. 30, 311-319 (2000).

319. Smeland, O. B. et al. Genetic Overlap between Schizophrenia and Volumes of Hippocampus, Putamen, and Intracranial Volume Indicates Shared Molecular Genetic Mechanisms. Schizophr. Bull. 44, 854-864 (2018).

320. Zhao, B. et al. GWAS of 19,629 individuals identifies novel genetic variants for regional brain volumes and refines their genetic co-architecture with cognitive and mental health traits. bioRxiv 586339, (2019).

321. Roth, G. \& Dicke, U. Evolution of the brain and intelligence. Trends Cogn. Sci. 9, 250 257 (2005).

322. Johnson, W., Bouchard, T. J., Krueger, R. F., McGue, M. \& Gottesman, I. I. Just one g: Consistent results from three test batteries. Intelligence 32, 319 (2004).

323. Davies, N. M., Holmes, M. V. \& Davey Smith, G. Reading Mendelian randomisation studies: A guide, glossary, and checklist for clinicians. BMJ 362, (2018).

324. Rebhan, M., Chalifa-Caspi, V., Prilusky, J. \& Lancet, D. GeneCards: a novel functional genomics compendium with automated data mining and query reformulation support. Bioinformatics 14, 656-664 (1998).

325. Firth, H. V. et al. DECIPHER: Database of Chromosomal Imbalance and Phenotype in Humans Using Ensembl Resources. Am. J. Hum. Genet. 84, 524-533 (2009). 
326. Grazioplene, R. G. et al. Subcortical intelligence: Caudate volume predicts IQ in healthy adults. Hum. Brain Mapp. 36, 1407-1416 (2015).

327. Bohlken, M. M. et al. Genes contributing to subcortical volumes and intellectual ability implicate the thalamus. Hum. Brain Mapp. 35, 2632-2642 (2014).

328. Sunkin, S. M. et al. Allen Brain Atlas: an integrated spatio-temporal portal for exploring the central nervous system. Nucleic Acids Res. 41, D996-D1008 (2012).

329. Wallace, C. et al. Statistical colocalization of monocyte gene expression and genetic risk variants for type 1 diabetes. Hum. Mol. Genet. 21, 2815-2824 (2012).

330. Yang, J., Lee, S. H., Goddard, M. E. \& Visscher, P. M. GCTA: a tool for genome-wide complex trait analysis. Am. J. Hum. Genet. 88, 76-82 (2011).

331. Westra, H.-J. et al. Systematic identification of trans-eQTLs as putative drivers of known disease associations. Nat. Genet. 45, 1238-1243 (2013).

332. Zhernakova, D. V et al. Identification of context-dependent expression quantitative trait loci in whole blood. Nat Genet 49, 139-145 (2017).

333. Macosko, E. Z. et al. Highly parallel genome-wide expression profiling of individual cells using nanoliter droplets. Cell 161, 1202-1214 (2015).

334. Newman, M. E. J. Modularity and community structure in networks. PNAS 103, $8577-$ 8582 (2006).

335. Bartholomeusz, H. H., Courchesne, E. \& Karns, C. M. Relationship Between Head Circumference and Brain Volume in Healthy Normal Toddlers, Children, and Adults. Neuropediatrics 33, 239-241 (2002).

336. Yang, J. et al. Genomic inflation factors under polygenic inheritance. Eur. J. Hum. Genet. 19, 807-812 (2011).

337. Martoglio, B. \& Golde, T. E. Intramembrane-cleaving aspartic proteases and disease: presenilins, signal peptide peptidase and their homologs. Hum. Mol. Genet. 12, R201R206 (2003).

338. Papadopoulou, A. A. et al. Signal Peptide Peptidase-Like 2c (SPPL2c) impairs vesicular transport and cleavage of SNARE proteins. EMBO Rep. e46451 (2019).

339. Maccioni, R. B. \& Cambiazo, V. Role of microtubule-associated proteins in the control of microtubule assembly. Physiol. Rev. 75, 835-864 (1995).

340. Myers, A. J. et al. The H1c haplotype at the MAPT locus is associated with Alzheimer's disease. Hum. Mol. Genet. 14, 2399-2404 (2005).

341. Coppola, G. et al. Evidence for a role of the rare p. A152T variant in MAPT in increasing the risk for FTD-spectrum and Alzheimer's diseases. Hum. Mol. Genet. 21, 3500-3512 (2012).

342. Desikan, R. S. et al. Genetic overlap between Alzheimer's disease and Parkinson's disease at the MAPT locus. Mol. Psychiatry 20, 1588 (2015).

343. Brunet, A. et al. Akt promotes cell survival by phosphorylating and inhibiting a Forkhead transcription factor. Cell 96, 857-868 (1999).

344. Medema, R. H., Kops, G. J. P. L., Bos, J. L. \& Burgering, B. M. T. AFX-like Forkhead transcription factors mediate cell-cycle regulation by Ras and PKB through p27 kip1. Nature 404, 782 (2000).

345. Carraway, K. L. et al. The erbB3 gene product is a receptor for heregulin. J. Biol. Chem. 269, 14303-14306 (1994).

346. Prigent, S. A. \& Gullick, W. J. Identification of c-erbB-3 binding sites for phosphatidylinositol 3'-kinase and SHC using an EGF receptor/c-erbB-3 chimera. EMBO J. 13, 2831-2841 (1994).

347. Liberzon, A. et al. Molecular signatures database (MSigDB) 3.0. Bioinformatics 27, 1739-1740 (2011).

348. Accili, D. \& Arden, K. C. FoxOs at the crossroads of cellular metabolism, differentiation, and transformation. Cell 117, 421-426 (2004).

349. Cross-disorder group of the Psychiatric Genomics Consortium. Genome wide meta- 
analysis identifies genomic relationships, novel loci, and pleiotropic mechanisms across eight psychiatric disorders. BioRxiv (2019).

350. Mallard, T. T. et al. Not just one P: Multivariate GWAS of psychiatric disorders and their cardinal symptoms reveal two dimensions of cross-cutting genetic liabilities. bioRxiv (2019).

351. Fried, E. I. The 52 symptoms of major depression: Lack of content overlap among seven common depression scales. J. Affect. Disord. 208, 191-197 (2017).

352. Smith, B. H. et al. Generation Scotland: the Scottish Family Health Study; a new resource for researching genes and heritability. BMC Med. Genet. 9, 1-9 (2006).

353. Smith, B. H. et al. Cohort Profile: Generation Scotland: Scottish Family Health Study (GS: SFHS). The study, its participants and their potential for genetic research on health and illness. Int. J. Epidemiol. 42, 689-700 (2013).

354. Scholtens, S. et al. Cohort Profile: LifeLines, a three-generation cohort study and biobank. Int. J. Epidemiol. 44, 1172-1180 (2015).

355. Fernandez-pujals, A. M. et al. Epidemiology and Heritability of Major Depressive Disorder, Stratified by Age of Onset, Sex , and Illness Course in Generation Scotland : Scottish Family Health Study ( GS : SFHS ). 75, 1-18 (2015).

356. Houle, D., Govindaraju, D. R. \& Omholt, S. Phenomics: the next challenge. Nat. Rev. Genet. 11, 855-66 (2010). 


\section{Peer-reviewed publications}

Nagel, M., Watanabe, K., Stringer, S., Posthuma, D., \& Sluis, S. (2018). Item-level analyses reveal genetic heterogeneity in neuroticism. Nature communications, 9(1), 905.

Nagel, M., Jansen, P. R., Stringer, S., Watanabe, K., De Leeuw, C. A., Bryois, J., Savage, J. E., Hammerschlag, A. R., Skene, N. G., Muñoz-Manchado, A. B., 23andMe Research Team, White, T., Tiemeier, H., Linnarsson, S., Hjerling-Leffler, J., Polderman, T. J. C., Sullivan, P. F., van der Sluis, S., \& Posthuma, D. (2018). Meta-analysis of genome-wide association studies for neuroticism in 449,484 individuals identifies novel genetic loci and pathways. Nature Genetics, 50(7), 920-927.

Savage, J. E., Jansen, P. R., Stringer, S., Watanabe, K., Bryois, J., De Leeuw, C. A., Nagel, M., Awasthi, S., Barr, P. B., Coleman, J. R. I., Grasby, K. L., Hammerschlag, A. R., Kaminski, J. A., ..., Dick, D. M., Heinz, A., Hjerling-Leffler, J., Ikram, M. A., Kendler, K. S., Martin, N. G., Medland, S. E., Pedersen, N. L., Plomin, R., Polderman, T. J. C., Ripke, S., van der Sluis, S., Sullivan, P. F., Vrieze, S. I., Wright, M. J., \& Posthuma, D. (2018). Genome-wide association meta-analysis in 269,867 individuals identifies new genetic and functional links to intelligence. Nature genetics, 50(7), 912-919.

Jansen, P. R., Watanabe, K., Stringer, S., Skene, N., Bryois, J., Hammerschlag, A. R., De Leeuw, C. A., Benjamins, J. S., Muñoz-Manchado, A. B., Nagel, M., Savage, J. E., Tiemeier, H., White, T., The 23andMe Research Team, Tung, J. Y., Hinds, D. A., Vacic, V., Wang, X., Sullivan, P. F., Van der Sluis, S., Polderman, T. J. C., Smit, A. B., Hjerling-Leffler, J., Van Someren, E. J. W., \& Posthuma, D. (2019). Genome-wide analysis of insomnia in 1,331,010 individuals identifies new risk loci and functional pathways. Nature genetics, 51(3), 394-403. 


\section{Nederlandse samenvatting}

Psychologische problemen zijn veelvoorkomend in de hedendaagse samenleving. Deze problemen variëren van milde stemmingsproblemen tot ernstige, soms chronische psychiatrische aandoeningen zoals depressie, schizofrenie en autisme spectrum stoornis. Ondanks tientallen jaren van onderzoek gericht op het achterhalen van de onderliggende biologische mechanismen zijn medische en psychologische behandelingen slechts gedeeltelijk succesvol. Over het algemeen wordt aangenomen dat alle karaktertrekken, zo ook psychiatrische aandoeningen en psychologische problemen, in ieder geval deels het gevolg zijn van de genen die we van onze ouders hebben mee gekregen. Het bestuderen van genetische informatie kan dan ook leiden tot een beter begrip van de biologische mechanismen die ten grondslag liggen aan geestelijke gezondheid, en zodoende van groot nut zijn voor patiënten.

De afgelopen twee decennia is het aantal studies dat kijkt naar genetische varianten gerelateerd aan allerlei karaktertrekken sterk toegenomen. Deze zogeheten 'genoombrede associatiestudies' (vaak afgekort als GWAS - genome-wide association study) stellen ons in staat om door het hele genoom (de complete genetische samenstelling van een mens) op zoek te gaan naar genetische varianten die, statistisch, gerelateerd zijn aan een karaktertrek naar keuze. Het aantal studies dat van deze techniek gebruikmaakt is sinds 2005 explosief toegenomen (https://www.ebi.ac.uk/gwas/diagram). Dit heeft een sterke invloed gehad op onze kennis van de biologie van ziekten, en heeft geleid tot de identificatie van duizenden genetische varianten geassocieerd aan honderden ziektes, psychiatrische aandoeningen en persoonlijkheidstrekken (breed gesteld: fenotypes).

Echter, voor een groot deel van het gedrag waarnaar onderzoek wordt gedaan, zoals psychiatrische aandoeningen, is het moeilijk om de gevonden genetische varianten te herleiden naar een duidelijk biologisch mechanisme. Wij zijn van mening dat dit deels het gevolg kan zijn van de manier waarop gedrag geoperationaliseerd wordt in genetische studies. Doorgaans worden deelnemers aan onderzoek geclassificeerd als 'cases' (als ze lijden aan een bepaalde stoornis) of 'controls' (als ze geen problemen ervaren). Echter, twee deelnemers die beiden geclassificeerd zijn als 'case' kunnen (deels) verschillende symptomen vertonen. Bijvoorbeeld: van twee personen met de diagnose 'depressie' kan de een last hebben van slaapgebrek, terwijl de ander een prima nachtrust heeft maar wel concentratieproblemen ervaart. Daarbij, is het goed denkbaar dat verschillende symptomen het gevolg zijn van verschillende genetische varianten. Door mensen met uiteenlopende symptomen, maar dezelfde diagnose, aan dezelfde groep toe te wijzen (i.e., cases) raak je dus informatie kwijt. Het is dan ook zeer belangrijk om goed na te denken over hoe het gedrag waarin men geïnteresseerd is gemeten wordt, aangezien dit van invloed is op de uitkomsten van genetische analyses.

In deze dissertatie maken we gebruik van grootschalige genetische data, en state-of-the-art methoden om te onderzoeken wat de potentiële voordelen zijn van het gebruik van meer gedetailleerde fenotypes (bijvoorbeeld symptomen in plaats van diagnoses) in genetisch onderzoek. 
Aangezien het effect van individuele genetische varianten op gedrag vaak zeer klein is, zijn grote steekproeven nodig om die effecten te kunnen detecteren. Uiteindelijk hopen we zo antwoord te kunnen geven op de vraag of karaktertrekken zoals we ze conceptualiseren op fenotypisch niveau ook te onderscheiden zijn op genetisch niveau. 


\section{Dankwoord}

Obtaining a PhD degree can be a lonely endeavor at times. However, it certainly isn't something that can be achieved without others. Here I'd like to express how thankful I am for all friends, family, co-workers and others that have helped me during my $\mathrm{PhD}$, positively distracted me from work every now and then, or that were always there in general.

Sophie, allereerst wil ik jou bedanken. Voor het vertrouwen dat je in me hebt gesteld door mij aan te nemen als PhD student op jouw VIDI beurs. De overgang van psychologie naar de genetica was even wennen, maar ik heb me vanaf het begin op mijn gemak gevoeld bij jou. Ik heb me, ook als er hobbels op de weg waren (het eerste project dat gescooped werd, revisies waarbij letterlijk alles opnieuw moest), begrepen gevoeld, en je gaf me het gevoel dat ik er nooit alleen voor stond. Je bent geduldig, heel erg betrokken, altijd bereikbaar, wetenschappelijk vlijmscherp, en waanzinnig snel met feedback geven. Ook op persoonlijk vlak kan ik het heel goed met je vinden, en dat draagt voor mij enorm bij aan het plezier dat ik heb gehad de afgelopen jaren. Ik heb natuurlijk weinig vergelijkingsmateriaal, dus jij zal het misschien statistisch niet heel sterk vinden, maar ik denk dat ik heel erg veel geluk heb gehad met jou als begeleider. Bedankt voor alles!

Danielle, gedurende mijn eerste jaar bespraken wij mijn werk misschien eens in de maand. Vanaf het moment dat de UKB een grotere rol ging spelen in mijn projecten werd dat contact intensiever. Ik heb bewondering voor hoe snel je tijdens meetings grip krijgt op wat ik aan het doen ben en waar ik tegenaan loop, en hoe je daar zo snel rake suggesties in kunt geven (ook al moest ik daar vaak even op wachten totdat jij en Sophie over de slappe lach heen waren). Jouw overzicht van het veld en kennis van zaken heeft een grote bijdrage geleverd aan mijn projecten. Naast je wetenschappelijke input waardeer ik heel erg hoeveel tijd en energie je hebt gestopt in het creëren van een fijne sfeer op de afdeling (borrels, uitjes, Sinterklaasgedichten, boot-feestjes). Ik heb de afdeling de laatste jaren zien uitgroeien naar een plek met een fijne werksfeer waar iedereen zich vrij voelt om bij elkaar binnen te lopen, en waar veel meer gebeurt dan werk alleen. Dank voor de mooie tijden!

I'd also like to express my gratitude to all members of my reading committee; Cathryn Lewis, Brenda Penninx, Eiko Fried, Huib Mansvelder and Michel Nivard. Thank you for taking an interest in my thesis.

Ik wil hier ook Han van der Maas niet vergeten, hoofd van de afdeling Psychologische Methodenleer ten tijde van mijn afstuderen. Jij hebt me met Sophie in contact gebracht, en daardoor is deze $\mathrm{PhD}$ op mijn pad gekomen. Ik herinner me nog goed dat jij tijdens mijn afstudeer ceremonie zei: “een beetje meer cum laude gedrag mag best!". Het is nog steeds niet mijn sterkste kant, maar mede dankzij jou kan ik me nu wel dr. noemen. 
Conor, bedankt voor je betrokkenheid bij mijn eerste project, maar ook voor de hulp, suggesties en tips van achter de schermen.

However much fun and inspiration working in science can be, I'm not the person to keep doing a job without being able to connect with the people around me. Fortunately, I met a lot of great people.

Soniá, Aná \& Vincent, thanks for all the fun times when the four of us were starting our PhD projects. The lab outing to Verona was amazing, as were all the times we had drinks at the VU.

Anke \& Christiaan, jullie waren er vanaf dag 1. We hebben slechts kort een kamer gedeeld, maar jullie zijn allebei op je eigen manier fantastisch! Anke, het was fijn om over van alles met jou te kunnen praten, ook de moeilijkere kanten van een $\mathrm{PhD}$ (of van het leven...). Ik voelde me snel geaccepteerd, en het was heel fijn dat ik, zeker in het begin, alles aan je kon vragen wat ik niet snapte (en dat was best veel). Christiaan, jou heb ik misschien nog wel méér vragen gesteld! Ik bewonder je kennis, en waardeer het enorm dat je er altijd voor open staat om mee te denken of mij je (altijd kritische) mening over weer een nieuwe methode te vertellen. Je doet nooit water bij de wijn (wel fallus-vormige ijsblokjes bij de whiskey... :).

Stephanie and Lisa, the two of you are bound to be mentioned in the same sentence. Thanks for being great roomies (not in the least because for some reason you always seemed to know the latest gossip). I could always count on you to stay until the end every time we had drinks (which was quite often). Thanks for sharing the $\mathrm{PhD}$ journey, hard times as well as fun times, with me!

Sven, ik heb veel van je geleerd, niet in de laatste plaats over het in perspectief plaatsen van werk en privé. Het was fijn om jou in de buurt te hebben gedurende een deel van mijn $\mathrm{PhD}$. Al werken we nu niet meer samen, ik hoop dat we elkaar zo nu en dan blijven zien!

Erdogan, jij brengt het nonchalante in de wetenschap waar veel mensen denk ik iets aan zouden hebben. Ik heb genoten van alle keren dat je 's ochtends met koffie mijn kamer binnen banjerde om even te kletsen. En nog veel meer van samen snowboarden, posters achter deuren plakken en samen wakker worden met Eye of the Tiger (al was jij meestal ruim voor mij op omdat je de receptionist moest lastigvallen met piano oefenen in de lobby of je poster nog even moest opfleuren met tipp-ex). Bedoeld of onbedoeld, je maakte me altijd aan het lachen!!

Iris, ik snap nu dat de shotjes die je me opdrong in Parijs waarschijnlijk payback waren voor het feit dat je in Boulder op de skipiste eindeloos, samen met Erdogan en mij, moest poseren voor een 'spring foto'. Fair enough.

Esther, good times! Bij elkaar binnenlopen als we even geen focus meer hadden, bespreken welke kleur het beste is voor je $R$ plotje (paars-roze, áltijd paars-roze), samen Stefpacking kijken en prosecco drinken op de VU in de vakantieperiode.

Philip, Jorim \& César, mijn eerste herinnering met jullie is Boulder 2015. Wat een fantastische trip! 
Jorim, bedankt voor al het videomateriaal dat je op de pistes van Loveland hebt gemaakt van Nicholas Magel, from the Netherlands! Onze airBnB in de wietplantage, door de bergen cruisen met de goPro uit de rijdende auto, het was te gek!

César, bedankt voor de hulp zo nu en dan op projecten, en alle fijne borrels en kroegavondjes op de lab outings.

Philip, mijn vaste kamergenoot op uitjes, cursussen en conferenties. We hebben een mooie tijd gehad! Ik ben blij dat jij er was om samen met mij dagen achtereen onduidelijke code te debuggen, analyses te herhalen en onmogelijke resultaten te plaatsen. Alleen had ik allang de handdoek in de ring gegooid. Het was mooi om je, naast als hele harde werker, ook te leren kennen als levensgenieter. Boulder onveilig maken, Kerstborrels (waar jij elke keer weer in een andere onesie verscheen), tot diep in de nacht staan stampen in een foute kroeg in Dublin, en nu al 2x een lekker zomers festivalletje. Ik ben blij om met jou als paranimf mijn $\mathrm{PhD}$ periode op de VU af te sluiten! \#lifeThug

Tinca, het was fijn om jou als collega te hebben. Je was altijd geïnteresseerd en betrokken, gezellig, en behulpzaam (bijvoorbeeld als er een presentatie gegeven moest worden en er een prijs op het spel stond).

Kyoko, CTG is not the same without you. You were always there (and I don't mean that as a figure of speech), and you knew the answer to all my questions. I'm glad we quickly figured out an arrangement where you answer all my questions and I drop you off home safely after you had a bit too much to drink. I miss you at the department, and I hope we'll get a chance to meet up in the States someday.

Eva, ik kan het vanaf het begin heel goed met je vinden. Je bent een goede luisteraar, (bijna) altijd vrolijk, en altijd in voor borrels, boulderen, schaatsen of lights festival (lees: kroegentocht). Thanks dat je zo'n fijne collega bent!

Josefin, Jeanne, Doug, Elleke, Maša, thanks for all that you bring to the lab. Whether it's general enthusiasm, dry humor, excellent city trip guiding skills, contagious laughter at the lunch table or partnership in climbing trees on our way back to the hotel in Nice. You're a great bunch! Eline, dank voor de gezelligheid, betrokkenheid en alle aandacht voor verjaardagen en andere festiviteiten!

Martijn, jouw enthousiasme is aanstekelijk. Ik hoop van harte dat we nog een keer samen een mooi netwerk project kunnen doen. Rory, Lianne, Dirk Jan, Yongbin \& Siemon, ik vind het super dat jullie allemaal (incl. tafelvoetbaltafel) naar Amsterdam zijn gekomen. De lunch is altijd lachen, jullie doen fascinerend werk, en de borrels en uitjes zijn nóg gezelliger geworden.

Although I mentioned many people separately, the greatest joy was all the times we were out together, whether it was talking and socializing at conferences, or roaming the streets of Antwerp, Dublin, Nice, Barcelona or Paris. Oh, and thanks for the speculoos. 
Tobias, Anne, Pieter, Geert, Oscar \& Emiel. Jullie hebben vooral zijdelings te maken gehad met mijn $\mathrm{PhD}$. Af en toe deed ik moeilijk over meegaan op een vakantie, of was ik te moe voor een feestje of de kroeg. Sorry daarvoor! Toch hebben jullie, misschien zonder je ervan bewust te zijn, bijgedragen aan de afronding van de PhD. Ik leefde toe naar, en op van, alle avonden, vakanties, en weekendjes Schier met jullie. Ik hou ervan hoe niemand zichzelf te serieus neemt, iedereen elkaar in de zeik neemt, maar er toch altijd is voor de ander als het erop aankomt. Deze paar jaar $\mathrm{PhD}$ was slechts een fase in mijn tijd met jullie, op naar de volgende era!

Tobias, ik mis de tijden dat we 3x per week met wat Amsteltjes en galetten aan de Amstel zaten. Ik kan altijd op je rekenen voor een driftige tirade als ik 'nee' zeg op je voorstellen om bier te gaan drinken in een café, of bij 'het grasje' in de zon te hangen en te zwemmen (lees: bier drinken). Af en toe kan ik zo'n terechtwijzing wel gebruiken om voor mezelf helder te krijgen wat er nou echt toe doet in het leven!

Anne, Annis, Nonchalanne, An Solo, ik vind het heel knap hoe je je in korte tijd vrijwel zelfstandig $R$ en Python hebt bijgebracht, en nu lekker als data scientist aan de slag bent. Ik ben blij dat je nu ook bent begonnen met boulderen, dan durf ik je beter even alleen te laten als we samen een festivalletje pakken en jij weer de hoogste mast in moet klimmen die je zo snel kunt vinden.

Pietro, MSc., je bent de Grippy pad onder mijn vrienden; altijd betrouwbaar en flexibel. Je bent onwijs sociaal, staat altijd klaar, en bent altijd in voor gezelligheid. Bovendien, welke vriendengroep kan er nou zonder een TOP-manager..?

Gurt, Papa G, ik heb bewondering voor hoe relaxed je in het leven staat. Dat we samen nog vele (mini)avonturen mogen beleven!

Oscar, bijna zelf klaar met je $\mathrm{PhD}$, lekker bezig hoor! Ik was blij toen ik hoorde dat je naar de VU kwam, eindelijk iemand van wie ik kon winnen met basketbal. Je hebt een gezonde kijk op de wetenschap, en weet goed wat je wil. Ik kijk uit naar de dag dat we als twee gedistingeerde, hooggeleerde doctors joelend vanaf de bank Overcooked spelen. Thanks dat je mij als paranimf wilde bijstaan!

Robert \& Alex, weinig paden lagen de afgelopen jaren zo dicht tegen het mijne aan als die van jullie. PML studeren op de UvA, lesgeven, allemaal aan de slag met een $\mathrm{PhD}$ project, bij elkaar om de hoek wonen in Oost, basketballen en nu ook klimmen. Ik ben blij dat ik alle ups en downs met jullie kon delen. Het samenwerken in de CC motiveerde me, en het was onwijs fijn om van elkaars ervaringen te kunnen leren. Thanks guys.

Lisanne, wijntjes drinken en onze AiO projecten met elkaar bespreken was altijd veel gezelliger dan het zo op papier klinkt. Ik ben blij dat jij en Alex nog met me wilden afspreken nadat ik was overgelopen naar de VU!

Steef, ouwe Limburger, je bent een topper ( $\mathrm{ja}$, je bent ook een fan van de Toppers, niemand is perfect). Bedankt voor je luisterend oor, en alle gezelligheid vanaf het moment dat we tijdens de Grondslagen colleges koffie zaten te drinken in Kriterion. Maar hou je nou eigenlijk van zachte of harde hoofdkussens...? 
Surya, Amber \& Oscar, het 'wetenschapsclubje' (poeh, dat klinkt suf!). Syl, jij hoort hier ook bij, als nieuwste erelid. Dank voor alle lange avonden bomen over wetenschap (althans, dat was de insteek, liep meestal anders), eindeloze dinertjes, overvloedige cocktails en wijntjes, en bovenal dat ik jullie ook tot mijn vrienden mag rekenen. Misschien moeten we onze club binnenkort eens omdopen, maar laten we vooral blijven doen wat we deden!

Rinse en Philip, ik lig altijd in een deuk met jullie. Dit boek is niet zo goed als het Matsboek, maar ik hoop dat jullie het toch de moeite waard vinden.

Basketbal was een belangrijke uitlaatklep tijdens de afgelopen jaren. Alexander, Felicia, Sjoerd, Ben, Vera, Roël, Ab ( ) ), Mike, Sabina, Kakin, Marco, Kinman, $\mathrm{COACH}$ en alle andere van het USC, ik heb genoten! Van slopende circuitjes (thanks Giuseppe!), toernooitjes in Leiden en BBQ's in het Flevopark tot onze overstap naar volwassenheid (lees: gevorderden training). Hopelijk zie ik jullie weer op het veld, of erbuiten!

Maarten en Ilse, ik heb me altijd zeer welkom gevoeld bij jullie. Bedankt voor de gastvrijheid in Buis, jullie betrokkenheid, en alle lekkere etentjes over de jaren.

Stan \& Tycho, mijn bonus broertjes. Ik ben blij dat wij elkaar blijven zien, of het nou in de boulderhal is (zeurend omdat we alle routes zo ellendig vinden), de kleine Cooldown in Amsterdam, een chic hotel in Vermont of met een slabbetje om in een Peruaans restaurant.

Zola, zusje, ook al doen we het te weinig, het is altijd fijn om tijd met jou door te brengen. Naast mijn werk eiste BB de laatste jaren ook flink wat tijd en aandacht op. Gelukkig kon jij me altijd voorzien van uitgebreid kattenadvies.

Keje, hermanito, onze levens zijn behoorlijk verschillend. Ik ben ontzettend blij dat ik je ben komen opzoeken in Mexico, en dat ik nu wat beter snap wat je daar zo trekt. Het was heel waardevol om daar samen tijd door te brengen. Waar je ook naartoe gaat in de toekomst, en met wie dan ook, ik kom langs.

Omdat je natuurlijk wel gewoon je dagelijkse portie aandacht nodig hebt, doen we even net alsof ik de hier volgende uitspraak zelf bedacht heb, in plaats van dat jij ' $m$ me per Whatsapp hebt toegestuurd: "Throughout my childhood there has been much struggle within my family due to a genetic trait failure within my brother's genome strings. This triggered me to execute a small scale GWAS on his neuroticism. Eventually this first-hand experience has brought me to where I am now, on the top of the mountain. This would never have been possible without my brother's neuroticism. He is doing well now. He managed to let go of his neurotic tendencies entirely (maybe he's even overdoing it a little)"

Ida en Jos, door jullie ben ik geworden wie ik ben. Het is makkelijk om jezelf te zijn als je weet dat je altijd kunt terugvallen op je ouders. Jullie zijn niet van de grote gebaren, maar jullie staan altijd klaar, en dat is niet vanzelfsprekend. Dank voor alle grote en kleine dingen. 
Tot slot, Inge. We hebben samen talloze bergen beklommen. Tijdens die momenten op de top, maar ook onderweg, voelde ik me altijd heel licht. In de bergen kan ik mijn hoofd pas echt leeg maken, en wordt het ineens makkelijk om de dagelijkse nonsens los te laten, en te zien wat er echt toe doet. Mijn beste herinneringen zijn die aan de keren dat we samen op avontuur uit gingen in de natuur.

Naast al die hoogtepunten hebben we ook beiden onze dalen gekend. Vaak konden we elkaar er doorheen slepen, maar we hebben er soms ook alleen voor gestaan. Ik weet dat we allebei alleen onze weg naar de top kunnen vinden, maar ik vind het toch leuker om het samen te doen. Dan maar niet de kortste weg. Wat er ook gebeurt, ik hou van jou. 



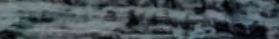
y 1 -

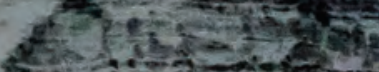

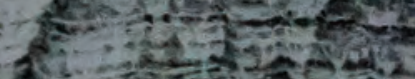

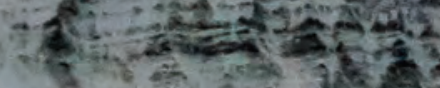
i. Cho a 35 a

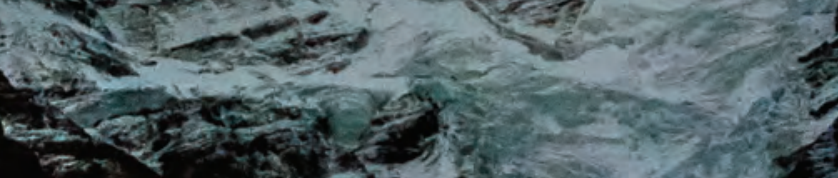
त
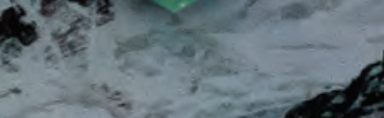


\section{UITNODIGING}

VOOR HET BIJWONEN VAN DE OPENBARE VERDEDIGING VAN MIJN PROEFSCHRIFT

\section{GHANGING PERSPECTIVES TOWARDS DETAILED PHENOTYPING IN GENETICS}

DINSDAG 30 JUNI 2020 OM 13.45 UUR

IN EEN ONLINE BIJEEKOMST VAN DE VRIJE UNIVERSITEIT

MEER INFO VOLGT PER E-MAIL

MATS NAGEL 